\title{
Daily and non-daily smokers
}

a profile of drive and cognitive control mechanisms

\author{
Bartlett, James E
}

Award date:

2020

Awarding institution:

Coventry University

Link to publication

\section{General rights}

Copyright and moral rights for the publications made accessible in the public portal are retained by the authors and/or other copyright owners and it is a condition of accessing publications that users recognise and abide by the legal requirements associated with these rights.

- Users may download and print one copy of this thesis for personal non-commercial research or study

- This thesis cannot be reproduced or quoted extensively from without first obtaining permission from the copyright holder(s)

- You may not further distribute the material or use it for any profit-making activity or commercial gain

- You may freely distribute the URL identifying the publication in the public portal

\section{Take down policy}

If you believe that this document breaches copyright please contact us providing details, and we will remove access to the work immediately and investigate your claim. 


\section{Daily and Non-daily Smokers: A Profile of Drive and Cognitive Control Mechanisms}

James E Bartlett

December 11, 2020

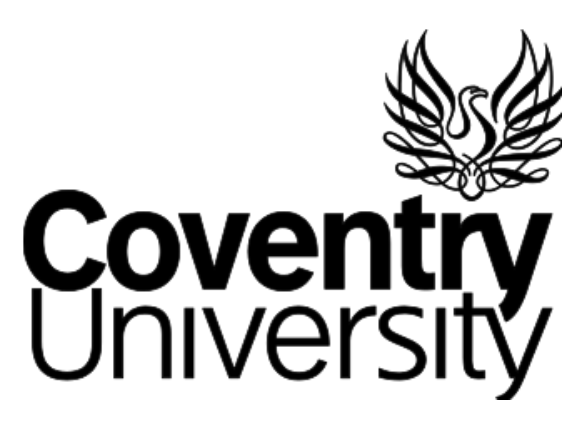

A thesis submitted in partial fulfilment of the University's requirements for the degree of Doctor of Philosophy

$$
\text { in the }
$$

School of Psychological, Social, and Behavioural Sciences

Word count: 58,230 
Content removed on data protection grounds 
Content removed on data protection grounds

Doctoral College and Centre for Research Capability and Development Last updated June 2017 
Content removed on data protection grounds

Doctoral College and Centre for Research Capability and Development Last updated June 2017 


\title{
Coventry
University
}

\section{Certificate of Ethical Approval}

Applicant:

\author{
James Bartlett
}

Project Title:

Attentional bias towards smoking cues in daily and non-daily smokers

This is to certify that the above named applicant has completed the Coventry University Ethical Approval process and their project has been confirmed and approved as Medium Risk

Date of approval:

01 March 2019

Project Reference Number:

P88261 


\section{Abstract}

Historically, smokers were considered a single homogeneous group, but over the past two decades research has increasingly focused on differentiating daily and non-daily smokers. Despite fundamentally different smoking habits and motives, daily and nondaily smokers have similar cessation rates. In order to understand why both groups may experience a similar difficulty quitting smoking, this thesis explored neurocognitive mechanisms associated with addictive behaviour. In order to profile these mechanisms, a systematic review was conducted, highlighting there was a gap to address in two areas of research relating to drive and control. Study One $(\mathrm{N}=60)$ and Study Two $(\mathrm{N}=166)$ investigated attentional bias towards smoking cues using the visual probe task, finding there was no meaningful difference between daily and non-daily smokers in trait-level attentional bias. Study Three $(\mathrm{N}=28)$ measured ERP components associated with inhibitory control (Go/NoGo task) and error processing (Eriksen Flanker task). There were no significant effects of interest, but the sample size was smaller than planned. This thesis made three contributions to the study of addictive behaviour. First, the systematic review highlighted that research investigating lighter and heavier smokers has a problematic level of heterogeneity in the definitions used to define the groups. Second, there was no meaningful difference in attentional bias between daily and non-daily smokers, supporting contemporary theories that attentional bias may be best conceptualised as a state-level construct. Finally, internal consistency estimates of the ERP measures of inhibitory control and error processing supported previous research reporting good psychometric properties. Overall, this thesis presented a focused profile of measures relating to drive and control neurocognitive mechanisms, but there were no meaningful differences between daily and non-daily smokers. If these mechanisms are important to addictive behaviour, future research will have to investigate their role using alternative designs. 


\section{Acknowledgements}

The biggest thank you goes to my family for putting up with me for the past five years, either locked away working on my PhD or talking about it when I should have been working. Bea was a constant source of support during the ups and downs and she has been there from the start of my university journey. My mum, Amanda, and grandparents, Doreen and Les, have been there from the beginning and without their support I may not have made it into an academic career. A big thank you also goes to my extended family, in particular my mother-in-law, Angela Nussey, who unfortunately passed away just before I completed my PhD. I always joked about how she would soon have to refer to me as Dr.

My supervisory team of Dr. Rebecca Jenks and Dr. Nigel Wilson were a great sounding board for my occasional erratic ideas and helped me to whittle my thesis into a coherent piece of work. Dr. Tim Jones was a valued member of my supervisory team until moving universities prevented his continued involvement. Thank you also goes to Dr. Hayley Wright and Dr. Miguel Farias who have been involved in my PhD at varying stages. Although I was an imposter in the psychology of religion and belief, the BBB lab helped me learn the ropes of academia. Without that research group, I would not have made friends in Sarah, Tommy, and Kyle.

Without the help of two people, my EEG study would not have been possible. Dr. Stoyan Kurtev spent hours working on $\mathrm{R}$ and Python programming with me and made the data analysis significantly less painful. Kieron Rooney spent hours of his time with me hooking participants up to the EEG equipment and tediously cleaning electrodes. What started as a research project turned into a valued friendship.

For the last couple of years, Arden University provided me with the trust to start my academic career. As well as funding the last stages of my $\mathrm{PhD}$, they provided me with the freedom to finish my thesis. Everyone on psychology island has been a great source of support, listening to my ranting and raving. However, particular thanks go to my past and present heads of school, Kara Johnson and Dr. Gail Steptoe-Warren. Their trust and support have allowed me to finish the thesis while I am still in one piece. 
I would like to thank my examiners Dr. Paul Christiansen and Dr. Kulbir Birak for a critical but friendly viva experience to mark the end of my PhD journey. They asked difficult questions, but they demonstrated a viva does not have to be intimidating.

Finally, those I have spent the largest number of hours with throughout this whole PhD have been my cats: Jonesy, Cleo, and Walter. Although they would often get in the way, they would be great sources of distraction and comfort after a long day's work. They have been with me since secondary school, so it is fitting they cap off the last stages of my education. 


\section{Contents}

Abstract iv

Acknowledgements $\quad$ v

1 Nicotine and Tobacco Use in Daily and Non-Daily Smokers 1

1.1 Introduction . . . . . . . . . . . . . . . . . . . 1

1.2 Smoking epidemiology . . . . . . . . . . . . . 3

1.2.1 Prevalence of nicotine and tobacco use $\ldots \ldots \ldots$

1.2.2 Risk factors for nicotine and tobacco use . . . . . . . . . . 4

1.3 The health implications of smoking . . . . . . . . . . . . 6

1.4 Nicotine Pharmacology . . . . . . . . . . . . . . . . 6

1.4.1 Pharmacokinetics . . . . . . . . . . . . . . . . 6

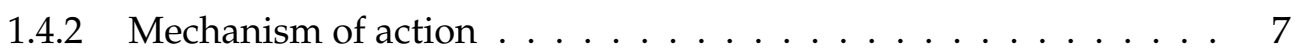

1.4.3 The mesolimbic dopamine pathway . . . . . . . . . . 8

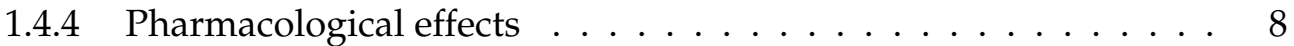

1.5 The rise of e-cigarettes $\ldots \ldots \ldots \ldots \ldots \ldots$

1.6 Daily and Non-Daily Smoking . . . . . . . . . . . . . . . . . 10

1.6.1 Definitions . . . . . . . . . . . . . . . . 10

1.6.2 Prevalence of daily and non-daily smoking . . . . . . . . . . 11

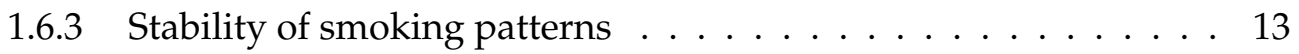

1.6 .4 Habits . . . . . . . . . . . . . . . . . . 14

1.6 .5 Motives . . . . . . . . . . . . . . . . . . . 15

1.6.6 Smoking cessation $\ldots \ldots \ldots \ldots \ldots$

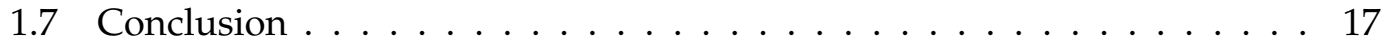

2 A Systematic Review of Neurocognitive Deficits 19

2.1 Chapter Overview . . . . . . . . . . . . . . . . . . . . . . . 19 


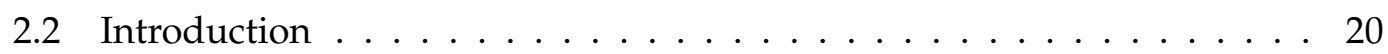

2.2.1 Neurocognitive mechanisms and addictive behaviour . . . . . 20

2.2.2 Previous reviews of non-daily smokers and neurocognitive mech-

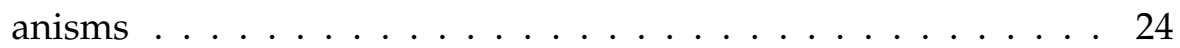

2.2.3 The current systematic review . . . . . . . . . . . 26

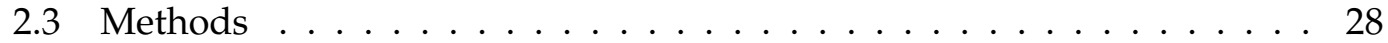

2.3 .1 Protocol and registration . . . . . . . . . . . . . . 28

2.3 .2 Eligibility criteria . . . . . . . . . . . . . 28

2.3.3 Information sources . . . . . . . . . . . . . . . . . . . . 29

2.3 .4 Search . . . . . . . . . . . . . . . . . . . . . . . . 29

2.3 .5 Study selection . . . . . . . . . . . . . . . 30

2.3.6 Data collection process . . . . . . . . . . . . . . . 30

2.4 Results . . . . . . . . . . . . . . . . . . . . 31

2.4 .1 Study selection $\ldots \ldots \ldots \ldots \ldots \ldots$

2.4 .2 Smoking labels and criteria . . . . . . . . . . . . . . 31

2.4 .3 Narrative review . . . . . . . . . . . . . . . . . . . . . 32

2.4.3.1 Executive network . . . . . . . . . . . . 33

2.4.3.2 Salience network . . . . . . . . . . . . . 35

2.4.3.3 Reward network . . . . . . . . . . . . . . . 40

2.4.3.4 Habit network . . . . . . . . . . . . . . 48

2.4.3.5 Self-directed network . . . . . . . . . . . . . . 49

2.4.3.6 Memory network . . . . . . . . . . . . . 50

2.5 Discussion . . . . . . . . . . . . . . . . . 53

2.5.1 Smoking labels and criteria $\ldots \ldots \ldots \ldots$

2.5 .2 Summary of findings . . . . . . . . . . . . . . . 55

2.5 .3 Integrative model . . . . . . . . . . . . . . . . . . . . . . 59

2.5 .4 Limitations . . . . . . . . . . . . . . . . 62

2.5 .5 Conclusion . . . . . . . . . . . . . . . . . 64

3 Attentional Bias Towards Smoking Cues 83

3.1 Chapter overview . . . . . . . . . . . . . . . 83

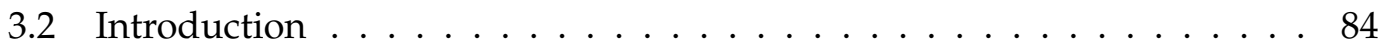


3.3 Study One . . . . . . . . . . . . . . . . . . . . 87

3.3 .1 Method . . . . . . . . . . . . . . . . . 87

3.3.1.1 Design .................... 87

3.3.1.2 Participants . . . . . . . . . . . . 87

3.3.1.3 Materials . . . . . . . . . . . . . . 88

3.3.1.4 Measures . . . . . . . . . . . . . . . 90

3.3.1.5 Procedure ...................... 94

3.3.1.6 Data analysis . . . . . . . . . . . . . . . 94

3.3.1.7 Data reduction . . . . . . . . . . . . . 95

3.3 .2 Results ... . . . . . . . . . . . . . . . . 95

3.3.2.1 Demographic characteristics . . . . . . . . . . . . 95

3.3.2.2 Control measures . . . . . . . . . . . . . . 96

3.3.2.3 Attentional bias . . . . . . . . . . . . . . . . 98

3.3.2.4 Reliability . . . . . . . . . . . . . . . . 99

3.3.2.5 Picture ratings . . . . . . . . . . . . . 100

3.3.3 Study One discussion . . . . . . . . . . . . . . . . . . . . . 101

3.4 Study Two . . . . . . . . . . . . . . . . . . . . 106

3.4 .1 Method . . . . . . . . . . . . . . . . . . . 106

3.4.1.1 Design ...................... 106

3.4.1.2 Participants and sample size calculation . . . . . . 106

3.4.1.3 Materials . . . . . . . . . . . . . . 107

3.4.1.4 Procedure . . . . . . . . . . . . . . . . . . . 109

3.4.1.5 Data analysis . . . . . . . . . . . . . . . 110

3.4.1.6 Data reduction . . . . . . . . . . . . . . 110

3.4 .2 Results . . . . . . . . . . . . . . . . . . 111

3.4.2.1 Demographic characteristics . . . . . . . . . . . . . 111

3.4.2.2 Attentional bias . . . . . . . . . . . . . 112

3.4.2.3 Reliability . . . . . . . . . . . . . . 120

3.4.3 Study Two discussion . . . . . . . . . . . . . . . . . . 120

3.4 .4 Meta-analysis . . . . . . . . . . . . . . . . . . . . 121

3.5 General discussion . . . . . . . . . . . . . . . . . . . 122

3.5.1 Strengths and limitations . . . . . . . . . . . . . 128 
3.5.2 Conclusion . . . . . . . . . . . . . . . . . . 131

4 Error Processing and Inhibitory Control 133

4.1 Introduction . . . . . . . . . . . . . . . 133

4.1.1 The association between cognitive control and risk of relapse . . 134

4.1.2 Inhibitory control and smoking . . . . . . . . . . . . . . 135

4.1.3 Error processing and smoking . . . . . . . . . . 136

4.1.4 The meaning of cognitive control deficits . . . . . . . . . 138

4.1.5 Cognitive control in daily and non-daily smokers . . . . . . . . 138

4.1.6 ERP components associated with inhibitory control . . . . . . . 140

4.1.7 ERP components associated with error processing . . . . . . . . 141

4.1 .8 Hypotheses . . . . . . . . . . . . . . . . . . . . . . . 142

4.2 Methods . . . . . . . . . . . . . . . . . . . . . . 142

$4.2 .1 \quad$ Participants . . . . . . . . . . . . . . . . . . . . 142

4.2 .2 Design . . . . . . . . . . . . . . . . . . . 143

4.2 .3 Materials . . . . . . . . . . . . . . . . . . . . . 144

4.2 .4 Measures . . . . . . . . . . . . . . . . . . . . . . . 144

4.2 .5 Procedure . . . . . . . . . . . . . . . . . . . . . . 147

4.2.6 EEG recording and processing . . . . . . . . . . . 148

4.2 .7 Data analysis . . . . . . . . . . . . . . . . . . . . 149

4.3 Results . . . . . . . . . . . . . . . . . . . . . . . . . . . 149

4.3 .1 Data screening . . . . . . . . . . . . . . . . . . . 149

4.3.1.1 Inhibitory control . . . . . . . . . . . . 149

4.3.1.2 Error processing . . . . . . . . . . . . . . . . 149

4.3 .2 Demographics . . . . . . . . . . . . . . . . . . . 150

4.3.3 Inhibitory control . . . . . . . . . . . . . . . . . . 150

4.3.3.1 Behavioural data . . . . . . . . . . . . . 150

4.3.3.2 ERP components . . . . . . . . . . . . . . . . 152

4.3 .4 Error processing . . . . . . . . . . . . . . . . . . 154

4.3.4.1 Behavioural data . . . . . . . . . . . . . . 154

4.3.4.2 ERP components . . . . . . . . . . . . . 156

4.3 .5 Reliability estimates . . . . . . . . . . . . . . . . . . 159 
4.4 Discussion . . . . . . . . . . . . . . . . . . . . . . . . 159

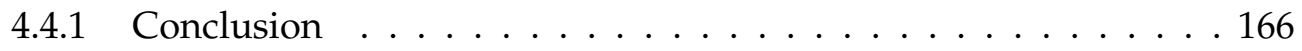

$\begin{array}{lll}5 & \text { General Discussion } & 168\end{array}$

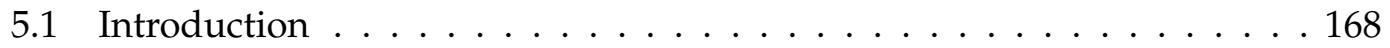

5.2 Heterogeneity of terminology for smoking groups . . . . . . . . . . 169

5.3 Attentional bias in daily and non-daily smokers . . . . . . . . . . . 171

5.4 Internal consistency of ERP measures of cognitive control . . . . . . . . 174

5.5 Limitations . . . . . . . . . . . . . . . . . . . . . . . 178

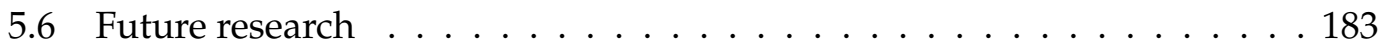

5.7 Conclusions . . . . . . . . . . . . . . . . . . 185

$\begin{array}{llr}6 & \text { References } & 188\end{array}$

$\begin{array}{ll}\text { A Systematic review search terms and strategy } & 226\end{array}$

$\begin{array}{lll}\text { B IAPS Images } & 228\end{array}$

$\begin{array}{ll}C \text { Ethics certificates } & \mathbf{2 3 0}\end{array}$

D R Packages and References $\quad 234$ 


\section{List of Figures}

3.1 Study One Visual Probe Task Diagram . . . . . . . . . . . . . . . 93

3.2 Study One nicotine dependence measures . . . . . . . . . . . . . . . 97

3.3 Study One attentional bias interaction plot . . . . . . . . . . . . . . 100

3.4 Study Two Visual Probe Task Diagram . . . . . . . . . . . . . . . . . . 109

3.5 Study Two nicotine dependence measures . . . . . . . . . . . . . . . 113

3.6 Study Two daily versus non-daily smokers interaction plot . . . . . . . 114

3.7 Study Two light versus heavy smokers interaction plot . . . . . . . . . 116

3.8 Study Two dependent versus non-dependent smokers interaction plot . 117

3.9 Study Two different attentional bias indexes . . . . . . . . . . . . 118

3.10 Mini meta-analysis forest plot . . . . . . . . . . . . . . . 122

4.1 Study Three task diagrams . . . . . . . . . . . . . . . . . . . . . 146

4.2 Study Three nicotine dependence measures . . . . . . . . . . . . . 152

4.3 Study Three Go/NoGo task behavioural results . . . . . . . . . . . . 153

4.4 Study Three Go/NoGo task grand average waveform . . . . . . . . . . 154

4.5 Study Three Go/NoGo task mean amplitude . . . . . . . . . . . . . . . 155

4.6 Study Three Eriksen Flanker task behavioural results . . . . . . . . . 156

4.7 Study Three Eriksen Flanker task grand average waveform . . . . . . . 157

4.8 Study Three Eriksen Flanker task mean amplitude . . . . . . . . . . . . 158 


\section{List of Tables}

2.1 Overview of systematic review study demographics. . . . . . . . . 67

2.1 Overview of systematic review study demographics. . . . . . . . . . . 68

2.1 Overview of systematic review study demographics. . . . . . . . . . . . 69

2.1 Overview of systematic review study demographics. . . . . . . . . . 70

2.1 Overview of systematic review study demographics. . . . . . . . . . . 71

2.1 Overview of systematic review study demographics. . . . . . . . . . 72

2.1 Overview of systematic review study demographics. . . . . . . . . . . 73

2.1 Overview of systematic review study demographics. . . . . . . . . . 74

2.2 Overview of systematic review studies smoking definitions and labels. . 75

2.2 Overview of systematic review studies smoking definitions and labels. . 76

2.2 Overview of systematic review studies smoking definitions and labels. . 77

2.2 Overview of systematic review studies smoking definitions and labels. . 78

2.2 Overview of systematic review studies smoking definitions and labels. . 79

2.2 Overview of systematic review studies smoking definitions and labels. . 80

2.2 Overview of systematic review studies smoking definitions and labels. . 81

2.2 Overview of systematic review studies smoking definitions and labels. . 82

3.1 Internal consistency estimates using Cronbach's alpha for each subscale of the WISDM-68. . . . . . . . . . . . . . . . . . . . . . 91

3.2 Study One mean (SD) values for participant characteristics and scale scores. . . . . . . . . . . . . . . . . . . 96

3.3 Mean $(S D)$ values for each picture rating attribute for each version of the visual probe task . . . . . . . . . . . . . . . . . . . . 101

3.4 Study Two mean $(S D)$ values for participant characteristics and scale scores. . . . . . . . . . . . . . . . . . . . . . . . . . 112 
4.1 Internal consistency estimates using Cronbach's alpha for each subscale of the WISDM-68. . . . . . . . . . . . . . . . . . . . 144

4.2 Study Three mean (SD) values for participant characteristics and scale scores. . . . . . . . . . . . . . . . . . . . . . . . . . . 151

B.1 IAPS image number and content label. The mean (SD) valence and arousal ratings presented for each image. . . . . . . . . . . . . . . 229 


\section{Chapter 1}

\section{Nicotine and Tobacco Use in Daily}

\section{and Non-Daily Smokers}

\subsection{Introduction}

The scientific community has known for over 60 years that smoking causes significant harm to public health (Thun and Henley 2010). There is an individual cost as fewer smokers feel in good health compared to non-smokers, and a collective cost where smoking is still the leading cause of preventable death in the UK, putting additional strain on the healthcare services (Action on Smoking and Health 2020). Despite the harmful consequences, when people decide they want to quit smoking, they find it incredibly difficult to remain abstinent long-term. Ninety percent of nicotine dependent smokers who attempt to quit relapse within 90 days (Tindle and Shiffman 2011; Herbec, Brown and West 2014). However, the majority of statistics on smoking describe smokers as if they were a homogeneous group. In reality, 11-33\% of smokers are estimated to be non-daily smokers, a sub-group characterised by their intermittent smoking (Coggins, Murrelle and Carchman 2009; Herbec et al. 2014). Despite exhibiting negligible signs of nicotine dependence and appearing to avoid experiencing nicotine withdrawal effects, these smokers find it nearly as difficult to quit smoking as daily smokers, with $82 \%$ of non-daily smokers relapsing within 90 days (Tindle and Shiffman 2011; Herbec et al. 2014). Despite exhibiting a similar difficulty to remain abstinent as daily smokers, non-daily smokers have only received marginal attention for what is influencing their smoking habits beyond the effects of nicotine dependence. 
Exploring factors associated with addictive behaviour in non-daily smokers is important as they are less likely to seek cessation assistance or receive specific quit advice from their healthcare provider (Tong, Ong and Vittinghoff 2006; Koontz, Harris and Okuyemi 2010). Therefore, it is important to explore the similarities and differences between daily and non-daily smokers in factors associated with addictive behaviour.

The factors that will be explored in this thesis are the neurocognitive mechanisms associated with addictive behaviour such as impulsivity and attentional bias. Deficits in these neurocognitive mechanisms can be broadly described as an increased drive to use drugs combined with the decreased ability to control drug use (Franken and van de Wetering 2015). Drive mechanisms are involved in the sensitivity towards rewards, and control mechanisms are related to concepts such as the ability to inhibit a response and learning from mistakes. Addictive behaviours are associated with deficits in these mechanisms that make drugs both desirable and hard to resist (Volkow, Wang and Fowler 2010), with studies showing that deficits are associated with greater risk of relapse during a quit attempt (Powell, Dawkins, and West 2009; Luijten et al. 2016). However, research investigating these deficits in dependent drug users often only compares them to a group of non-using healthy controls. A large body of research has focused on these neurocognitive mechanisms and how they can contribute to maintaining drug use in daily smokers (for general reviews including sections on nicotine dependence, see Luijten et al. 2014; Zilverstand et al. 2018), but non-daily smokers have received relatively little attention. This means there is a gap in research to explore the similarities and differences between daily and non-daily smokers on neurocognitive mechanisms associated with addictive behaviour.

The overall aim of this thesis is to profile these neurocognitive mechanisms in daily and non-daily smokers. There are two main components for how this thesis will make an original contribution to the field of addictive behaviour. First, there are isolated areas of research focusing on daily and non-daily smokers, but there is no known previous systematic review of neurocognitive mechanisms drawing them together. Therefore, Chapter Two will present a systematic review of previous research investigating deficits in neurocognitive mechanisms in daily and non-daily smokers. As a preview of the results, the review highlights two main areas of interest stemming from conflicting past research or a lack of research. The first area relates to attentional 
bias as a drive mechanism promoting drug use, and the second area relates to cognitive control (inhibitory control and error processing) as mechanisms controlling drug use. Chapter Three presents two studies on attentional bias and Chapter Four has one study exploring inhibitory control and error processing in daily and non-daily smokers.

Chapter One will present the context around nicotine and tobacco use, and highlight why it is an important area of research considering the public health implications of daily and non-daily smoking. The chapter begins by establishing the prevalence and health implications of smoking to highlight the need for research to discover what contributes towards problematic drug use. The pharmacology of nicotine and tobacco will then be briefly outlined to establish how it produces its rewarding effects. A profile of daily and non-daily smokers will then be presented to emphasise the individual differences between these types of smokers. This will begin by outlining some of the difficulties in defining the groups, and what the differences are in their smoking habits and motives. Chapter One will end with an outline of the similarities in cessation failure rates between daily and non-daily smokers to highlight why investigating a wider range of smokers is an important area of research, providing the rationale for the remainder of this thesis.

\subsection{Smoking epidemiology}

\subsubsection{Prevalence of nicotine and tobacco use}

The average smoking prevalence for those over the age of 15 is estimated at $21 \%$ globally, but this figure can be split into $36 \%$ in men and $7 \%$ in women (World Health Organisation 2015). In the United Kingdom, smoking prevalence in the population was estimated at 15\% (West, Kale and Kock 2020b). Broken down into age groups, the prevalence of smoking in the United Kingdom is the lowest in those over the age of 60 (8\%) and between the ages of 50 and 59 (18\%; Action on Smoking and Health 2020). The highest rates of smoking were in those between the ages of 16 and 24 (20\%), 35 and $49(21 \%)$ and 25 and $34(22 \%)$. 


\subsubsection{Risk factors for nicotine and tobacco use}

Adolescence is a critical period for somebody beginning to smoke, with one study reporting that $33 \%$ of daily smokers had their first cigarette at the age of 13 or below, and $43 \%$ when they were between the ages of 14 and 16 (Breslau and Peterson 1996). Furthermore, Breslau and Peterson (1996) found that an earlier age of smoking initiation is associated with a higher rate of relapse later in life. Siqueira, Diab and Bodian (2000) found similar results with adolescents who smoked regularly tended to begin smoking around the age of 13 , but those who only experimented with cigarettes started later around the age of 15 . However, experimentation can still lead to frequent smoking behaviour as a meta-analysis of prevalence estimates found that for those that ever try smoking, two thirds will progress onto a pattern of daily smoking (Birge, Duffy and Miler 2017). This suggests that early teenage years are a particularly vulnerable period for smoking initiation, and there appears to be a number of important risk factors during this time.

The familial environment that an adolescent grows up in has an impact on later smoking behaviour. Garcia-Rodriquez, Blanco, and Wall (2014) found that a family history of drug use, experiencing sexual abuse, and social deviance were particularly important for predicting smoking initiation. This suggests that negative life experiences during adolescence may contribute to the risk of starting to smoke. This is supported by a large twin study showing that social-environmental factors have an important contribution to smoking initiation (Kendler, Schmitt and Aggen 2008). Early adolescence is vulnerable to the effect of familial environment as it can account for approximately $50 \%$ of the variance in the liability of smoking initiation. This is thought to be mediated by familial influences that persist through to later life such as the development of attitudes towards drug use. However, the influence of the familial environment declines during later adolescence and disappears by the age of 30 (Kendler et al. 2008). In later life, biological factors such as genetics appear to be more important. Genetics influences the neurobiology of an individual which controls how drugs are metabolised and impacts their subsequent effects. For example, candidate gene studies have identified that there is a relationship between smoking quantity and particular variants of a gene that encodes enzymes that metabolise nicotine (Ware and 
Munafó 2015). Kendler et al. (2008) found that genetic factors have negligible influence in early adolescence, but increase steadily throughout life to explain $60 \%$ of the variance in smoking liability by the age of 30 . This suggests that genetics and the familial environment have a complicated relationship, and have diverging patterns of influence throughout adolescence and into later life.

In addition to adolescent life experiences, there are several key risk factors that are related to smoking. First, there is a high rate of comorbidity between smoking and mental health problems. One large population study in the United States reported that people with mental health problems smoke $44 \%$ of the total number of cigarettes smoked (Lasser, Boyd and Woolhandler 2000), and smoking is believed to be the most common comorbid behaviour in psychiatric patients (Stahl 2013). Second, there is a robust relationship between smoking and personality traits. Munafó, Zetteler, and Clark (2007) conducted a meta-analysis that suggested high extraversion and neuroticism was associated with the increased likelihood of being a smoker. This is supported by a larger, more recent meta-analysis on nearly 80,000 people who replicated the findings with the addition of smoking being associated with lower conscientiousness (Hakulinen, Hintsanen, and Munafó 2015). Neurotic personality traits may be associated with smoking in adulthood due to an increase in anxiety, stress, and negative affect incidences. Smokers often hold the belief that cigarettes improve their mood and reduce stress (West 2009), meaning they might smoke as a form of selfmedication. A large review concluded that on a population level, smokers do indeed report greater stress and negative affect than non-smokers (Kassel, Stroud and Paronis 2003). However, there is no clear direct relationship between smoking initiation and negative affect. There is evidence to suggest that it is related to smoking maintenance in adulthood, but there is no clear explanation for how negative affect might influence smoking initiation. Overall, these studies demonstrate that there are different influences that can affect the likelihood of someone starting to smoke, and these influences can be diverging in childhood and adulthood. It is important to understand these different influences as smoking can have harmful health implications. 


\subsection{The health implications of smoking}

The evidence for smoking causing lung cancer reached wide-spread acceptance in the 1950s (Thun and Henley 2010). Recent statistics estimate that smoking accounts for $80 \%$ of all incidences of lung cancer in males and 50\% in females (Jemal, Bray and Center 2011). Smoking also increases the incidences of other kinds of cancer and cardiovascular disease (Hecht 2010; World Health Organisation 2015). Non-daily smoking was historically seen as a positive step as reducing the number of cigarettes smoked per day was perceived to be a less harmful alternative to daily smoking (Okuyemi et al. 2002). However, non-daily smoking is associated with several negative health outcomes. Lung cancer follows a dose-response relationship where smoking more cigarettes is associated with an increased risk of developing lung cancer (Schane et al. 2010). This means that non-daily smokers have an intermediary risk between nonsmokers and daily smokers. However, the risk for developing cardiovascular disease is almost identical for daily and non-daily smokers (Schane et al. 2010). In addition, non-daily smoking is associated with a greater number of emergency room visits (Tong et al. 2006) and increased mortality in comparison to non-smokers (Løchen, Gram and Mannsverk 2017). These studies show that non-daily smoking is far from risk-free and the health and economic implications means that it is important to better understand why both daily and non-daily smokers struggle to stop smoking longterm.

\subsection{Nicotine Pharmacology}

\subsubsection{Pharmacokinetics}

Nicotine is the substance found in tobacco that is responsible for its rewarding properties (Henningfield and Benowitz 2010). Nicotine can be absorbed through many areas of the body including the lungs, nasal mucus, the skin, and intestines (Advokat, Comaty and Julien 2014). This allows for several different types of tobacco product, with absorption through the lungs being the most common in the form of cigarette smoke. Nicotine can also be absorbed through the nasal mucus in the form of snus 
(a powdered smokeless form of tobacco popular in Scandinavia), or through the gastrointestinal tract in the form of chewing tobacco (Advokat et al. 2014). However, this thesis focuses on cigarette smoking, with other forms of tobacco outside its scope. This is due to cigarettes being the most popular form of tobacco product across the world and the majority of the research base has focused on cigarettes (West 2017). When cigarette smoke is absorbed through the lungs, the arterial blood supply is saturated with nicotine and rapidly travels to the brain (Benowitz 2010). From the average cigarette, this results in $0.5-3 \mathrm{mg}$ of nicotine being absorbed (Advokat et al. 2014). Once this is present, the elimination half-life of nicotine is approximately two hours before being metabolised into its primary metabolite, cotinine, which has a longer half-life of up to 20 hours (Henningfield and Benowitz 2010). Relating to the half-life of nicotine, the average daily smoker enters a state of nicotine withdrawal two hours after their last cigarette and these withdrawal effects can last for weeks, but reach a peak intensity approximately 48 hours after cessation (Advokat et al. 2014).

\subsubsection{Mechanism of action}

Nicotine produces a wide range of effects through the central and peripheral nervous system (Advokat et al. 2014). It is an agonist that can bind directly to specific subtypes of acetylcholine receptors known as nicotinic receptors (Stahl 2013). These subtypes appear to consist of two main variants $\left(\alpha^{4} \beta^{2}\right.$ and $\left.\alpha^{7}\right)$ that influence dopamine release to produce the rewarding effects of nicotine in three main ways (Stahl 2013). The first acts on the post-synaptic $\alpha^{4} \beta^{2}$ receptors that regulates dopamine directly into the nucleus accumbens. The second pathway is through the presynaptic $\alpha^{7}$ receptor on glutamate neurons that initiates dopamine release. The third pathway desensitises the post-synaptic $\alpha^{4} \beta^{2}$ receptors on GABA neurons which prevents the inhibition of dopamine release and increases the levels of dopamine in the nucleus accumbens. Together, these are the main mechanisms of action that nicotine uses to increase the level of dopamine, and the mesolimbic pathway is particularly affected. 


\subsubsection{The mesolimbic dopamine pathway}

The mesolimbic dopamine pathway is the neural basis for reinforcement and reward in the brain, and is usually stimulated by natural rewards such as sex, food, and feelings of accomplishment (Stahl 2013). Drugs with addictive properties produce their effects by bypassing endogenous neurotransmitters, and directly bind to areas in this pathway that mediate rewarding behaviour. The pathway consists of dopamine projections from the ventral tegmental area and the nucleus accumbens, and extend to the prefrontal cortex, amygdala, and hippocampus (Advokat et al. 2014). The nucleus accumbens is particularly important for nicotine dependence and consists of two complementary sub-structures: the shell and the core. The rewarding properties of smoking originate from dopamine being released into the shell of the nucleus accumbens through the activation of nicotinic receptors (Balfour 2004). The rewarding effect of this large dopamine release in the shell results in the delivery of nicotine being associated with the behaviours and stimuli related to the delivery such as opening a pack of cigarettes or clicking a lighter (Balfour 2008). On the other hand, the core of the nucleus accumbens has a complementary role as it mediates the effects of sensory and environmental cues that are related to drug-seeking behaviour (Balfour 2008). Repeated administration of nicotine increases the release of dopamine within the nucleus accumbens core, and this is posited to result in the compulsive response to sensory and environmental cues that stimulate drug-seeking behaviour. The combination of these two structures results in cues that are related to the delivery of nicotine being able to elicit a rewarding response independently, and the behaviour that leads up to the delivery of nicotine becomes habitual and compulsive.

\subsubsection{Pharmacological effects}

One of the most noticeable aspects of nicotine inhaled through tobacco smoke is the initial rush from the first few puffs of a cigarette. This is caused by the initial blood reaching the brain being saturated with nicotine (Adkovat et al. 2014). Nicotine is classified as a stimulant and produces dose-related psychoactive effects (Henningfield and Benowitz 2010). For example, one review reported that high nicotine content cigarettes produced a greater euphoric effect and head rush than low nicotine content 
cigarettes (Kalman 2002). However, cigarettes containing high doses of nicotine were also related to feelings of tension and anxiety. In terms of the psychological effects, there is some evidence to suggest that nicotine can enhance aspects of memory and attention (Nees 2015). Nicotine can also produce physical effects by reducing weight gain (Advokat et al. 2014). These factors are important for the motivational aspects of addiction as smokers commonly hold the belief that cigarettes can help to manage their weight, stress, and concentration (West 2009). Although these are the common pharmacological effects of nicotine, some smokers place greater importance on different aspects of smoking. Smokers are commonly characterised as one single group, with their individual differences ignored. Daily and non-daily smokers report different motives behind their substance use and will display fundamentally different smoking behaviour.

\subsection{The rise of e-cigarettes}

Cigarette smokers are the population of interest in this thesis, but the rise of e-cigarettes as a standalone smoking habit or dual use with cigarettes should be acknowledged. In comparison to an estimated $15 \%$ of adults who smoke cigarettes (West et al. 2020b), $7 \%$ of UK adults use an e-cigarette based on a representative household survey (West, Beard and Kale 2020a). There is some cross-over in smoking behaviour as many smokers tend to use both an e-cigarette and tobacco cigarettes. There are an estimated $49 \%$ of occasional e-cigarette users and $42 \%$ of daily e-cigarette users who also smoke cigarettes (West et al 2020a). Relatedly, 22\% of smokers also use an e-cigarette. This suggests that there is a large amount of cross-over in smoking behaviour, where many are dual-users of e-cigarette and tobacco cigarettes.

This is important as e-cigarettes have the potential to help smokers quit smoking altogether or replace cigarettes as a less risky alternative. In a sample of US adults, $35 \%$ of smokers who tried quitting in the previous three months substituted some cigarettes with an e-cigarette (Chaffee, Watkins, and Glantz 2018). There is a positive association between e-cigarette prevalence and successful quit rates (Beard, West, and Michie 2020) and daily e-cigarette use is linked to greater odds of successful smoking abstinence across two years (Kalkhoran, Chang, and Rigotti 2020). This suggests that 
switching to an e-cigarette can lead to greater smoking cessation outcomes. It is crucial that e-cigarettes do not just add to smoking behaviour though. Non-daily e-cigarette use is not associated with greater odds of successful smoking abstinence (Kalkhoran et al. 2020) and dual-using cigarettes and e-cigarettes does not lead to a reduction in exposure to tobacco smoke and the health risks associated with cigarette smoking (Wang, Olgin, and Nah 2018). This shows that e-cigarettes have the potential to replace cigarettes as a less risky alternative, but it is important that dual users are not just supplementing their smoking behaviour, rather than focusing on sole e-cigarette use or abstinence.

The rise of e-cigarettes in the smoking landscape is important to acknowledge as many smokers are dual users and they have the potential to be a less risky alternative to cigarettes. However, given that cigarette smoking is still the most prevalent smoking behaviour (West et al. 2020b) and the majority of the pre-existing smoking research has focused on cigarettes (West 2017), the population of interest in this thesis remains cigarette smokers. As e-cigarettes continue to be established as a core smoking habit, it will be important to consider their long-term effects in future research.

\subsection{Daily and Non-Daily Smoking}

\subsubsection{Definitions}

To retain consistency in terminology throughout this thesis, this section will start by outlining the common definitions that are used to differentiate different smoking groups. The terms daily and non-daily smoking are popular as they are consistent across studies and can be ascertained simply through asking whether someone smokes daily or less than daily (Husten 2009). Light and heavy smoking is one of the most common measures and relates to levels of cigarette use, but it is associated with the most wide ranging criteria across different studies (Fagan and Rigotti 2009). Smokers have been labelled as dependent or not dependent on nicotine based on self-report measures of nicotine dependence such as the Fagerström Test of Cigarette Dependence (FTCD; Heatherton, Kozlowski and Frecker 1991; Fagerström 2011). Splitting smokers into different groups has some merit as Shiffman, Ferguson, and Dunbar (2012b) 
showed that you can differentiate between smokers with above $90 \%$ accuracy when focusing on nicotine dependence.

Throughout this thesis, the different smoking groups are referred to as daily and non-daily smokers. Husten (2009) identified this categorisation as the most useful as daily versus non-daily smoking behaviour is the most stable across time. Smoking frequency fluctuates over time, but daily and non-daily smoking patterns appear to be the most consistent. Moreover, differentiating daily and non-daily smokers closely resembles the questions in large population studies. It is important to remain consistent as if each study uses its own criteria, it makes it difficult to compare results as differences may be due to the composition of the groups being different. However, in Study One (Chapter Three), smokers were categorised based on FTCD scores as a group of light and heavy smokers. This was chosen at the time to be consistent with previous research on attentional bias in different smoking groups. After the first study, this approach was deemed to be problematic and the criteria changed for the final two studies. The FTCD dichotomy was retained for the first study as detailed information on the number of smoking days per month was not available and it was not possible to reclassify smokers as daily and non-daily smokers.

\subsubsection{Prevalence of daily and non-daily smoking}

Across a range of western countries, non-daily smokes form the minority of smokers and daily smokers form the majority. In the United Kingdom, $36 \%$ of smokers were classified as non-daily smokers in comparison to $64 \%$ of daily smokers (Kotz, Fidler and West 2012). Similar trends can be found across the European Union and in the United States. Averaged across all EU member states (as of 2010), 82\% of the smoking population consisted of daily smokers and $18 \%$ identified as non-daily smokers (Bogdanovica, Godfrey and McNeil 2011). In the United States, non-daily smokers formed a slightly lower proportion with an estimate of $13 \%$, in contrast to $87 \%$ being classified as daily smokers (Tindle and Shiffman 2011). These figures show that there is a similar trend across western developed countries. On the other hand, non-daily smoking has historically been the most prevalent smoking pattern in developing countries and ethnic minority groups within developed countries (Tong et al. 2006; Fagan and Rigotti 2009; Levy, Biener and Rigotti 2009; Schane et al. 2009; Shiffman 2009). In a 
large representative survey of Brazilian households, daily smokers formed just $40 \%$ of the smoking population as opposed to $60 \%$ as non-daily smokers (Dutra, Formagini, and Pinto 2017). Together this shows that in different regions and communities, the proportion of daily and non-daily smokers may differ.

To add another layer of complexity, the proportion of non-daily smokers has steadily increased over time. Using a large representative sample of the United States, Pierce et al. (2009) showed that the proportion of smokers described as non-daily increased from 1992 to 2002 in all age groups, but the largest increase was $4.7 \%$ to $6 \%$ of $18-29$ year olds. Correspondingly, there was a $4 \%$ decrease across all age groups during the same time period for those who smoked 15 or more cigarettes per day. Kotz et al. (2012) found similar evidence in the United Kingdom as the number of non-daily smokers increased from $26 \%$ of smokers in 2006 to $36 \%$ in 2011 . This shows that daily and non-daily smokers form two sizeable groups that deserve attention, and as the proportion of non-daily smokers continues to increase, it is important to understand their similarities and differences to daily smokers.

One problem with estimating the prevalence of non-daily smoking is they may be frequently underreported as some non-daily smokers do not identify as a smoker (Levinson, Campo and Gascoigne 2007; Koontz et al. 2010; Brown et al. 2011; Shiffman, Tindle and Li 2012c; Robertson et al. 2015). Large surveys of college students have shown that less than half of the students who admit to smoking will identify as being a smoker (Levinson et al. 2007). This dissonance has been explored by qualitative research as deniers believe the term is reserved for those who smoke every day and smoke a substantial number of cigarettes (Brown et al. 2011). This shows that non-daily smoking may be underestimated in some population estimates as if they are directly asked whether they are a smoker, a large proportion of non-daily smokers may not have that identity and more nuanced questioning is required.

Large studies that can provide stable population estimates have identified that non-daily smoking is associated with several characteristics. There is converging evidence that in comparison to daily smokers, non-daily smokers are more likely to be female, younger, from a higher socio-economic background, and have a higher level of education (Levy et al. 2009; Schane, Glantz and Ling 2009; Kotz et al. 2012; Shiffman et al. 2012c; Løchen et al. 2017). This is in addition to the higher prevalence in ethnic 
minority groups (Fagan and Rigotti 2009; Levy et al. 2009). This shows that non-daily smoking is more likely in some populations.

As tobacco control measures become stricter and smoking bans are more widespread, the number of non-daily smokers is estimated to increase (Fagan and Rigotti 2009). Evidence of this can already be seen in the United Kingdom as the number of non-daily smokers increased from $26 \%$ of smokers in 2006, to $36 \%$ in 2011 (Kotz et al. 2012). Considering this, it is important to understand the similarities and differences between daily and non-daily smokers in terms of their progression over time and profile.

\subsubsection{Stability of smoking patterns}

Historically, non-daily smokers were largely dismissed as a stable smoking group due to the perception that it was only seen in young, inexperienced smokers who would soon progress onto heavier levels of smoking (Shiffman 2009). However, a large proportion of smokers maintain a consistently low level of consumption for many years. The non-daily smokers in Shiffman et al. (2012b) had been smoking a consistently low number of cigarettes for 18 years, and in Shiffman et al. (2012c) they had smoked an estimated 42,000 cigarettes across their lifetime. In a longitudinal study across a fouryear follow-up period, very light non-daily smokers were the second most consistent smoking group behind heavy daily smokers (Levy et al. 2009). This shows that nondaily smokers are an interesting group as they are comfortable in maintaining a low rate of consumption, despite historical preconceptions.

Another layer of complexity to different smoking groups is that they are not rigid; people can transition between smoking groups. Hoeppner, Bidwell, and Colby (2014) found that in a group of university smokers, there were five different smoking trajectories across an academic year. There were three stable groups of frequent and occasional smokers, but two groups either increased or decreased their smoking pattern. Although this shows how some smokers transition between smoking patterns, this study is limited by the short time frame of the study and the young age of the smokers. Stronger evidence comes from a longitudinal cohort study that found between the ages of 21 and 38, some smokers transitioned between being a lighter and heavier smoker (Robertson et al. 2015). Group membership was fluid and people transitioned between daily, non-daily, and non-smoking groups. This evidence suggests 
that the historic perception that non-daily smokers were only a transitionary group on the road to becoming a daily smoker is inaccurate. Non-daily smokers can maintain a stable pattern of infrequent use, and smokers can fluctuate to and from heavier patterns of use throughout their life.

\subsubsection{Habits}

One area where there are clear differences between daily and non-daily smokers is in their smoking habits. Non-daily smokers consistently smoke a small number of cigarettes on smoking days with the average ranging from four to seven (Shiffman et al. 2012c; Shiffman et al. 2012b; Herbec et al. 2014). The range fluctuates in each study as there were different criteria used to define the groups. Non-daily smokers are largely free of dependence with negligible scores on several different measures of nicotine dependence (Shiffman et al. 2012b). This allows them to engage in voluntary days of abstinence. One study found that non-daily smokers only smoke on four days per week, but there is a large amount of variation as this figure ranged from four to twenty-seven smoking days per month (Shiffman et al. 2012c). This shows that nondaily smokers are characterised by their low and infrequent smoking pattern.

Daily smokers rarely go days without smoking and tend to smoke an average of fifteen per day, ranging from five to thirty cigarettes (Shiffman, Dunbar and Scholl 2012a; Shiffman et al. 2012b). They are characterised by nicotine dependence and their smoking behaviour is largely governed by the two-hour half-life of nicotine. Nicotine dependence is known to have a large genetic component, and how people metabolise nicotine appears to be particularly important (Ware and Munafó 2015). A large-scale meta-analysis of genome-wide association studies found that some gene variants related to enzymes that metabolise nicotine were related to the number of cigarettes smoked per day (Thorgeirsson, Gudbjartsson and Surakka 2010). This means that if people possessed particular alleles, it was associated with them smoking more cigarettes per day. However, the effects are tiny and appear to only be visible nearing a population scale. There is also no information provided on whether non-daily or nondependent smokers were included, but as nicotine dependence was defined as scoring four or more on the FTCD, the sample likely consisted of daily smokers. When 
smaller studies have specifically compared daily and non-daily smokers on their ability to metabolise nicotine, there have been no significant differences (Shiffman, Dunbar and Benowitz 2014a). Although there may be differences in nicotine metabolism on a population scale, directly comparing daily and non-daily smokers provides evidence against one hypothesis that non-daily smokers can maintain themselves on a small number of cigarettes due to being active in the body for longer. These findings show that there are fundamental differences in the smoking habits of daily and nondaily smokers, although there does not appear to be a substantial difference in nicotine metabolism.

\subsubsection{Motives}

In addition to differences in the habits of daily and non-daily smokers, there are qualitative differences in their motives for smoking. Soon after they have initiated smoking in adolescence, different trajectories of smoking behaviour can be identified in daily and non-daily smokers. Despite smoking an overall smaller number of cigarettes in comparison to adult smokers, lighter and heavier adolescent smokers begin to report different motives for smoking (Rubinstein et al. 2014). Lighter smokers cited reporting to smoke when socialising as the most important factor, whereas heavier smokers tended to smoke when they felt angry. This is also reflected in qualitative research as university age non-daily smokers reported drinking alcohol and the socialising opportunities relating to smoking as their most important motives (Brown et al. 2011). This suggests that despite smoking a relatively small number of lifetime cigarettes, different smoking trajectories can already be differentiated in adolescent smokers. Studies have shown that adolescence is a particularly important period for smoking initiation (Breslau and Peterson 1996; Siqueira et al. 2000). As smokers get older and smoke for longer periods of time, these differences widen and create more distinct groups.

Daily smoking in adulthood was classically defined as "trough maintenance" as cigarettes are used frequently throughout the day to maintain a relatively constant level of nicotine relating back to its two-hour half-life (Russell 1971). When daily smokers are asked to report what motivates them to smoke, they report factors relating to the physical indicators of dependence such as tolerance, craving, habit, and loss of control over smoking opportunities (Shiffman et al. 2012a). These factors relating 
to dependence appear to be particularly robust as they are consistent even when potential confounding variables such as education and ethnicity are taken into account (Scheuermann, Nollen and Cox 2015). This suggests that the physical indicators of nicotine dependence are particularly important for daily smokers, and continuing to smoke is largely to avoid experiencing withdrawal effects.

On the other hand, adult non-daily smokers report very different motives behind their smoking behaviour. Non-daily smokers have been described as indulgent smokers, as they tend to smoke in specific situations to enhance an already pleasurable experience (Shiffman, Dunbar and Li 2014b). Consistent with adolescent non-daily smokers, adults report factors such as social situations, exposure to specific cues, and pleasurable sensory properties as important (Shiffman et al. 2012a). The primary reasons behind their smoking behaviour can also change depending on the situation they are in (Darlow and Lobel 2012). In contrast to daily smokers, non-daily smokers can report fluctuating motives behind their smoking behaviour, and their smoking rate decreases when they do not feel like smoking. This shows that non-daily smokers report to smoke in response to particular cues and are not locked into a ritualised habit like daily smokers. Non-daily smokers can often be described as "social smokers" who only smoke around bars and alcohol. There does appear to be a special relationship between smoking and drinking alcohol, and the relationship is particularly pronounced in non-daily smokers (Tong et al. 2006; Hoeppner et al. 2014). However, the majority of non-daily smokers' cigarettes are smoked within the confines of their own home which shows that there is some intrinsic pleasure derived from smoking (Shiffman et al. 2014b). This collection of research demonstrates that adult non-daily smokers are characterised by their tendency to smoke for the pleasure they receive from it or in response to specific cues.

\subsubsection{Smoking cessation}

Despite the differences between daily and non-daily smokers, one crucial similarity is their apparent difficulty to quit smoking. Both daily and non-daily smokers find it incredibly difficult to quit smoking long-term with $77-92 \%$ of daily smokers and $74-83 \%$ of non-daily smokers relapsing within 90 days of a quit attempt (Tindle and Shiffman 2011; Kotz et al. 2012; Herbec et al. 2014). Furthermore, for a group of 
very light daily smokers (1-5 cigarettes per day), 24 out of 41 (59\%) relapsed within a ten-day incentivised cessation programme (Ashe and Wilson 2020). Considering that non-daily smokers often engage in voluntary days of abstinence while daily smokers start to experience nicotine withdrawal symptoms two hours after their last cigarette, these figures are remarkably close.

Smoking cessation in non-daily smokers has often been overlooked as clinical trials often set stringent inclusion criteria focusing on daily smokers (Shiffman 2009). Of the few studies that have been conducted to understand the cessation characteristics of non-daily smokers, they are more confident in their ability to quit smoking and often report a greater intention to quit smoking (Cooper, Taylor and Murray 2010; Schauer, Malarcher and Berg 2014). They may be more confident of their ability to quit smoking, but they are less likely to seek assistance when doing so (Tong et al. 2006; Schauer et al. 2014). This may be the result of being used to having short-term control over their smoking behaviour, and they expect to be able to have the same control over their long-term smoking behaviour and quit independently. In addition to being less likely to seek assistance, non-daily smokers are less likely to receive cessation advice from their healthcare provider (Tong et al. 2006; Koontz et al. 2010). Nondaily smokers may create a barrier for themselves in their lack of smoking identity as those who admit to being dishonest in their description of their smoking behaviour are seven times less likely to receive specific advice than those who accurately report their smoking behaviour (Koontz et al. 2010). Therefore, there may be a problematic combination of non-daily smokers not seeking smoking cessation assistance and receiving less support from healthcare professionals who may not perceive them to be in need of assistance. Despite differences in their smoking habits and motives, daily and non-daily smokers both find it difficult to quit smoking long-term.

\subsection{Conclusion}

In summary, non-daily smokers maintain an infrequent but consistent smoking pattern. In comparison to daily smokers, they smoke less than daily with a fraction of the number of cigarettes per smoking day. Furthermore, daily and non-daily smokers report different reasons for why they smoke. Daily smokers appear to smoke habitually 
to maintain their nicotine level, while non-daily smokers are influenced by personal situations and smoke for the enjoyment they receive from it. Crucially however, nondaily smokers exhibit few signs of nicotine dependence but find it almost as difficult to quit smoking long-term as daily smokers. As both daily and non-daily smokers find it difficult to quit smoking, it is important to understand the factors beyond nicotine dependence that are potentially related to this difficulty. The factors that this thesis focuses on are the neurocognitive mechanisms that have been associated with problematic drug use.

Neurocognitive mechanisms such as impulsivity and attentional bias have been explored as factors associated with addictive behaviour, and they have promising uses as markers of the risk of relapse (Franken and van de Wetering 2015). Studies have demonstrated that deficits in these mechanisms are associated with greater risk of relapse during a quit attempt (Powell, Dawkins, and West 2009; Luijten et al. 2016). However, the majority of past research investigating neurocognitive deficits compares dependent drug users with a group of non-using healthy controls. A large body of research has focused on these neurocognitive mechanisms and how they can contribute to maintaining drug use in daily smokers, but non-daily smokers have received relatively little attention. Therefore, it is important to explore the similarities and differences between daily and non-daily smokers as deficits in neurocognitive mechanisms may contribute to why both smoking groups find it difficult to quit smoking longterm. In order to document what previous research there is on this topic, Chapter Two presents a systematic review of previous research investigating these mechanisms in daily and non-daily smokers. 


\section{Chapter 2}

\section{A Systematic Review of Neurocognitive Deficits}

\subsection{Chapter Overview}

Chapter One outlined the differences between daily and non-daily smokers in their habits and motives. Despite fundamental differences in their smoking behaviour, daily and non-daily smokers report similar difficulties in quitting smoking (Tindle and Shiffman 2011; Kotz et al. 2012; Herbec et al. 2014). One group of factors associated with problematic drug use are neurocognitive mechanisms such as error processing and inhibitory control. There is a large body of evidence identifying deficits in these mechanisms when comparing drug-dependent users to non-users (for a review, see Zilverstand et al. 2018), but research on non-dependent users has received substantially less attention, and there does not appear to be a comprehensive review that has synthesised the existing published literature.

The introduction to Chapter Two begins with an overview of the theoretical models explaining the role of these neurocognitive mechanisms associated with problematic drug use. There have been systematic reviews focusing on daily and non-daily smokers and neurocognitive mechanisms in isolation, but there has not been a systematic review on how daily and non-daily smokers differ on these mechanisms. The results of the systematic review begin in Section 2.4 and outline previous research organised into themes of different neurocognitive mechanisms. This review targets an important gap in the literature as neurocognitive deficits associated with addictive behaviour may explain the similarity in low long-term smoking cessation rates between 
daily and non-daily smokers. The results of this systematic review inform the remainder of this thesis. Chapters Three and Four present empirical research to target some of the gaps and inconsistencies in current knowledge identified in the review.

\subsection{Introduction}

Between $77 \%-92 \%$ of daily smokers and $74 \%-83 \%$ of non-daily smokers relapse within 90 days of a quit attempt (Tindle and Shiffman 2011; Kotz et al. 2012; Herbec et al. 2014). Despite fundamental differences in their smoking habits, motives, and severity of nicotine dependence (Shiffman et al. 2012a; 2012b; 2014b), both daily and non-daily smokers find it difficult to quit smoking long-term. It is surprising that non-daily smokers struggle to maintain long-term control over their smoking behaviour when they are characterised by their short-term control over their smoking behaviour. This short-term control usually allows them to engage in voluntary days of abstinence and being less likely to smoke when they do not feel like it. This contrasts with the behaviour of daily smokers who are characterised by their habitual smoking behaviour. As all forms of smoking are associated with severe health implications (Tong et al. 2006; Schane et al. 2010; Løchen et al. 2017), it is important to better understand the contributing factors towards maintaining drug use and subsequent relapse in order to develop more effective interventions. Neurocognitive mechanisms have been associated with problematic drug use and they may be promising in improving the diagnosis and treatment of addictive behaviour (Franken and van der Wetering 2015). Therefore, this chapter presents a systematic review to collate the research to date on neurocognitive mechanisms in daily and non-daily smokers.

\subsubsection{Neurocognitive mechanisms and addictive behaviour}

Neurocognitive mechanisms are the underlying neural processes such as impulsivity and reward processing that are related to the characteristics of addictive behaviour. For example, addicted populations continue to use drugs despite adverse consequences such as affecting health or family life. This lack of awareness suggests a deficit in decision making (Yücel and Lubman 2007). These mechanisms can be understood to be one of the lowest levels of human motivation (West 2009). In the PRIME theory of 
human motivation in the context of addiction, there are five levels of motivation from simple reflex responses that all animals possess, to the human ability to plan ahead and form mental representations of potential actions (West and Brown 2013). The neurocognitive mechanisms that are typically investigated make up the bottom two levels of the PRIME theory. The first level of responses refer to starting or stopping an action. The second level of impulses and inhibitions influence or control the urges to start an action (West and Brown 2013). Most neurocognitive models of addiction have focused on these two complimentary factors.

Impulses, also known as drive mechanisms, are related to the motivation to use a drug and encompass craving and the valuation of rewards. The role of impulses in addiction has been emphasised in the Incentive Sensitisation Theory (Robinson and Berridge 1993; 2003; 2008). This theory describes how with increasing drug use, the brain becomes sensitised and motivation switches from enjoying drugs to wanting drugs. The implications of this switch is that drugs and cues associated with the drugs become salient, and the attention of drug users is drawn towards them, also known as attentional bias.

On the other hand, there are inhibitions or control mechanisms. These mechanisms control behaviour and include impulsivity and error processing. The role of control in addiction has been emphasised in models that highlight impaired impulsivity as a hallmark of addiction as it creates a vulnerability for drug dependence (VerdejoGarcía et al. 2008). Addictive behaviour has been conceptualised as the transition from habitual behaviour to compulsive behaviour (Everitt and Robbins 2016). This means that there is less control, hinting at deficits in decision-making when drug use continues despite adverse consequences. Verdejo-García et al. (2008) suggests that impulsive behaviour is pre-existing in high risk individuals such as children who have substance dependent parents. However, it is unlikely that only one of these mechanisms is acting at one time. Addictive behaviour is characterised by the combination of a decreased ability to control drug use while simultaneously having an increased urge to do so. There are different theories that combine the opposing influences of drive and control. For example, the PRIME theory (West and Brown 2013) includes impulses and inhibitions in human motivation. For the purposes of this thesis, the 
theoretical framework is based on the impaired response inhibition and salience attribution (iRISA) syndrome model (Goldstein and Volkow 2002; 2011). In their review of translating findings from neuroscience to being applied to interventions, Franken and van der Wetering (2015) organised their recommendations using the iRISA model. As the content of this thesis is focused on the role of neurocognitive deficits in addictive behaviour, the iRISA model will be used to organise the findings here too.

The iRISA model describes how impaired prefrontal cortex function explains a syndrome of behaviours that characterise addiction (Goldstein and Volkow 2002). When drug users have transitioned to a state of addiction, drug-related cues are salient and non drug-related rewards are devalued. Simultaneously, the ability to control and monitor behaviour is impaired, meaning that drug seeking and drug taking can become out of control which is characteristic of a cycle of drug taking, abstinence, and relapse (Goldstein and Volkow 2011). In addition to the articles outlining the model, several reviews have periodically reviewed the evidence of deficits in neurocognitive mechanisms in addiction. In a review of 26 imaging studies focusing on stimulant dependence, there was support for the iRISA model (Crunelle et al. 2012). In comparison to healthy controls, substance dependent participants demonstrated impairment in drive mechanisms such as under valuing non drug-related rewards, and in control mechanisms such as delay discounting where short-term gains are sought despite long-term consequences. A more recent systematic review of studies investigating neurocognitive mechanisms used stringent inclusion criteria (Zilverstand et al. 2018). In order to provide an update of the evidence supporting the iRISA model, only studies that contained a healthy control group, group samples sizes above 15 , and imaging studies that controlled for false discovery rates were included in the review. Consistent with previous reviews, the evidence was consistent with the iRISA model. Studies reported that drug dependent participants had impaired cognitive function in comparison to healthy controls, and showed increased activity when viewing drugrelated cues, but a blunted response during non-drug related tasks, consistent with previous evidence in drive mechanisms. Likewise for control mechanisms, drug users made more risky choices on the Iowa gambling task, and displayed impaired response inhibition. Together, these reviews summarise a wide range of studies, and the results have largely supported the predictions outlined in the iRISA model for neurocognitive 
mechanisms in addiction.

The iRISA model describes drug users who have already reached a state of addiction and compares them with healthy non-drug using controls. These reviews (Goldstein and Volkow 2002; Zilverstand et al. 2018) have not explored non-daily or nondependent substance users in any detail. In the update to the iRISA model (Goldstein and Volkow 2011), there is a brief mention of the distinction between dependent and non-dependent drug users as there should be a shift in the valuation of drug and non drug-related rewards. In addicted users, there should be decreased sensitivity to nondrug related rewards in comparison to non-addicted users (Goldstein and Volkow 2011). In addition, when transitioning from a non-dependent to dependent user, negative reinforcement such as withdrawal should be the predominant motivating factor in comparison to positive reinforcement and the benefits from using drugs (Everitt and Robbins 2016). This is consistent with research in smoking motives in daily and non-daily smokers, as non-daily smokers report smoking to enhance pleasurable experiences whereas daily smokers report smoking to avoid experiencing withdrawal symptoms (Shiffman et al. 2012a; Shiffman et al. 2014b). Beyond the predictions in the iRISA model on reward processing, very few studies reported in these reviews have focused on the distinction between drug users with different levels of dependence. One exception is Goudriaan et al. (2010) who focused on cue reactivity in smokers. There was not a significant increase in activity when heavy smokers viewed smoking images in contrast to healthy controls. However, when sub-group analyses were performed and the smokers were divided into a group of higher and lower dependence smokers, high dependence smokers produced greater activity in the prefrontal cortex and anterior cingulate cortex when viewing smoking images in comparison to both healthy controls and lower dependence smokers. This suggests that cue reactivity towards smoking images is more evident in smokers with higher nicotine dependence, supporting the iRISA model prediction that drug-related rewards are highly valued. However, the smokers may have been divided into high and low nicotine dependence groups based on the self-report FTCD, but the inclusion criteria for smokers was smoking 15 or more cigarettes per day (Goudriaan et al. 2010). Therefore, these were two groups of daily smokers, but one was relatively heavier than the other. This 
does not tell us anything about neurocognitive mechanisms associated with addiction in non-dependent or non-daily users. In order to understand how impairments in neurocognitive mechanisms may differ between daily and non-daily smokers, it is important to review studies with wider inclusion criteria.

\subsubsection{Previous reviews of non-daily smokers and neurocognitive mecha- nisms}

Historically, non-daily smokers have only received limited empirical investigation which may be due to the perception that they were just in a transitionary period on the road to becoming a daily smoker (Shiffman 2009). Several reviews have focused on the differences in demographic characteristics and the health impact of smoking in non-daily smokers. Okuyemi et al. (2002) performed one of the earliest reviews on non-daily smokers. They explored the definitions used to identify light or nondaily smokers and provided an overview of prevalence estimates of non-daily smokers. Okyemi et al. (2002) concluded that it was an under-researched area despite studies already demonstrating that light smoking was still associated with adverse health implications. Coggins et al. (2009) produced a more comprehensive review and summarised that there are clear differences in the smoking habits, motives, and personalities of daily and non-daily smokers. Finally, Schane et al. (2010) conducted an extensive review of the health effects of non-daily smoking. They concluded that non-daily and daily smoking is associated with a similar increase in the risk of cardiovascular disease, but lung cancer followed a dose-response relationship with the severity of nicotine dependence. Higher smoking rates were associated with greater risk of developing lung cancer. These reviews provide a comprehensive overview of the demographics and smoking habits of daily and non-daily smokers, but there is little attention to what factors may be influencing their smoking behaviour, such as neurocognitive mechanisms associated with problematic drug use.

In order to investigate how neurocognitive mechanisms may be associated with the smoking behaviour of daily and non-daily smokers, it may be best to focus more generally on reviews focusing on neurocognitive mechanisms. These reviews had a broader focus on different drug using populations, rather than focusing on one particular drug. In a systematic review on stimulant dependence (cocaine, amphetamine, 
ecstasy, and nicotine) and neuropsychological functioning, Crunelle et al. (2012) found that drug users displayed reduced activation in regions associated with impulsivity (anterior cingulate cortex), attentional bias (limbic regions), and decision making (prefrontal cortex). Luijten, Machielsen and Veltman (2014) produced a systematic review on EEG and fMRI research in the specific domains of inhibitory control and error processing, encompassing key control mechanisms. They found that smokers in comparison to non-smokers showed inhibitory control deficits through reduced ERPs and lower levels of activation in areas such as the anterior cingulate cortex. However, there were few studies on smokers for each process with six and four studies for inhibitory control and error processing respectively. Smith, Mattick and Jamadar (2014) performed a systematic review and meta-analysis on the relationship between behavioural inhibition and drug use. Across different drugs, Smith et al. (2014) concluded that drug users show greater deficits in behavioural inhibition than non-drug users. However, their sub-group analysis for studies focusing on nicotine dependence showed that smokers had greater deficits than non-smokers when the study used a Go/NoGo task, but not when they used a stop-signal task. However, this may be due to greater variability in results and a smaller sample of studies as the meta-analytic effect size was only slightly smaller than the Go/NoGo task. The most relevant systematic review and meta-analysis in terms of population focused on research into the different facets of impulsive personality traits specifically in cigarette smokers (Kale, Stautz, and Cooper 2018). They found that there was a small positive relationship between impulsivity personality traits and smoking status, meaning that higher scores were associated with being a smoker in comparison to a non-smoker. This shows that there is evidence that smoking is associated with greater personality measures of impulsivity. However, these findings cannot be directly compared to the other reviews as personality measures of impulsivity only have a small relationship with behavioural measures of impulsivity (Cyders and Coskunpinar 2011). Nevertheless, there is converging evidence that smokers display deficits in behavioural and personality measures of impulsivity in addition to error processing. This means that smokers tend to be more impulsive, find it more difficult to inhibit responses, and are slower to learn from making mistakes than non-smokers.

A salient feature of all these reviews is that they only focused on studies comparing 
drug users with a non-using control group. None of these reviews included studies with an inclusive sample of daily and non-daily smokers. Two of the studies did report some exploratory correlational analyses. Smith et al. (2014) reported that some studies found that the severity of drug use was associated with more severe deficits. Kale et al. (2018) found that impulsivity was associated with greater levels of nicotine dependence. However, the problem with only including a correlational measure of drug use severity is these studies often include stringent inclusion criteria for drug users, such as the previous example of Goudriaan et al. (2010) who only included those who smoked 15 cigarettes per day or more. This means that the studies were restricted to regular, dependent drug users, and non-dependent or non-daily users were excluded by design. Therefore, it is important to identify research that has specifically sampled non-dependent or non-daily smokers and compared them to daily smokers.

\subsubsection{The current systematic review}

Most of these reviews provided a comprehensive overview of neurocognitive mechanisms across a range of drug users. This is useful for the generalisability of how drug use is associated with neurocognitive deficits. However, there is a gap in research for considering how these neurocognitive mechanisms differ between daily and nondaily smokers. Previous reviews have covered each element of this separately. There are studies that have taken an extensive look at the differences between daily and non-daily smokers in terms of their demographics and smoking behaviour. There are also reviews that have focused on neurocognitive deficits across a broad range of drug users, but are limited to comparing users and non-users. Therefore, there is a gap in what is known about the presence of neurocognitive deficits in daily and non-daily smokers. If deficits in these neurocognitive mechanisms are important for understanding the maintenance of drug use, collating previous research in daily and non-daily smokers may be crucial to understanding why they find it similarly difficult to quit smoking long-term.

The primary aim of this systematic review is to collate all the previously published literature that has compared a group of lighter and heavier smokers on the neurocognitive deficits associated with substance use. Smith et al. (2014) highlighted in their systematic review of behavioural inhibition that studies have produced mixed 
evidence for either a positive association between drug use and deficit severity, or a lack of association. Therefore, it will be interesting to evaluate the consistency of this conclusion when studies are included that have explicitly sampled non-dependent or non-daily smokers. One of the limitations of previous studies including a correlation between the severity of nicotine dependence and a measure associated with neurocognitive mechanisms, is that there is usually strict inclusion criteria limiting the sample to moderate to highly dependent smokers. This means that there may only be a positive correlation between nicotine dependence and the severity of these deficits once the smokers have crossed a threshold into habitual smoking behaviour.

A secondary aim of this review is to highlight the inconsistency of the definitions used to classify daily and non-daily smokers. Until this point, smoking groups were referred to as daily and non-daily smokers, but different studies have used a range of labels and criteria. During the presentation of the results, the description of each study uses the label the original authors used. The heterogeneity of terminology and definitions is problematic for comparing results across studies in each area as any difference in results between two studies may stem from heterogeneity in defining their smoking groups. This is not to deny the complexity of smoking behaviour. Nicotine dependence exists along a continuum, but it is valid to dichotomise smokers into a group of more and less dependent smokers (Shiffman et al. 2012b). The aim of documenting the variety of smoking definitions in the studies included in this review is to echo the concerns of Husten (2009) who previously highlighted the numerous definitions for non-daily smokers.

From a theoretical perspective, it appears that there may be two potential patterns of results. The consensus appears to be a positive relationship between the severity of nicotine dependence and deficits in neurocognitive functioning. There is some evidence of this in previous reviews as Smith et al. (2014) found mixed results for a positive relationship between drug dependence and deficit severity, and Kale et al. (2018) found a positive relationship between personality measures of impulsivity and nicotine dependence. This pattern of results would support the findings that non-daily smokers are relatively more successful quitting smoking and feel more confident doing so (Cooper et al. 2010; Schauer et al. 2014). Alternatively, another potential pattern 
is that non-daily smokers possess certain characteristics that protect them from transitioning into nicotine dependence (Rass, Fridberg, and O'Donnell 2014). Where daily smokers consistently show deficits in neurocognitive processes in comparison to nonsmokers, non-daily smokers may outperform both non-smokers and daily smokers which allows them to maintain control over a small but consistent pattern of smoking behaviour. This would challenge the assumption that these neurocognitive deficits are important in maintaining substance use and relapse. If non-daily smokers do not show any deficits but still struggle to quit smoking long-term, it may signal that deficits in neurocognitive mechanisms may not be critical in relapse behaviour. Therefore, a systematic review of the existing research may help to elucidate which pattern of results has received the most support.

\subsection{Methods}

\subsubsection{Protocol and registration}

The systematic review was performed according to the preferred reporting items for systematic reviews and meta-analyses (PRISMA) guidelines (Moher, Shamseer, and Clarke 2015). A protocol for performing the review was registered on the international prospective register of systematic reviews (PROSPERO) database (registration number: 42016048393). An additional protocol can be found on the Open Science Framework (https://osf.io/cjdkq/).

\subsubsection{Eligibility criteria}

To be included in the review, studies had to meet the following eligibility criteria: (1) involve adult human participants (age 18 or above), (2) compare two or more groups of lighter and heavier smokers, and (3) focus on cigarette smoking (excluding other forms of tobacco such as water pipe or chewing tobacco). In addition, studies that investigated the effect of nicotine on cognitive performance were excluded. This was due to the aims focusing on how different smoking groups differ on aspects of cognitive performance, not on how the pharmacological effects of nicotine affect cognitive 
performance. There are studies included in the review which manipulate deprivation or administer neurotransmitter receptor agonists. The difference between studies excluded and included relate to the direction of influence. Studies administering nicotine focus on its effects on cognitive mechanisms, but the focus here is on comparing different smoking groups on these cognitive mechanisms. The different smoking groups were the main focus point but studies using deprivation and receptor agonists compared their effects relative to normal behaviour, as opposed to being interested in the effects of nicotine directly. The study characteristics included being written in English and published in a peer-reviewed journal. Due to the lack of previous reviews on this topic, there was no lower bound to the year that a study was published in.

As there were no previous reviews with the focus of outlining differences in cognitive deficits between smoking groups, the outcome measures of interest were deliberately wide-ranging. Studies that included any measure of cognitive function associated with substance use were included which encompassed measures such as: behavioural tasks, eye-tracking, event-related potential (ERP) or EEG methods, and brain imaging studies using PET or fMRI.

\subsubsection{Information sources}

Three databases were searched between 28/09/2016 and 21/11/2016. An updated search was performed between $2 / 1 / 2019$ and 6/2/2019 to cover any research since the initial review. Medline, PsychInfo and Scopus were searched between these dates. A notable deviation from the protocol and registration was that Web of Science was also intended to be searched. However, university access issues prevented this database from being included in the review.

\subsubsection{Search}

The search process was performed by combining key words relating to nicotine dependence with key words relating to cognitive deficits. A series of key words relating to smoking and nicotine dependence were created. These were divided into three types: light smoking, heavy smoking, and nicotine dependence. This was due to the inconsistency of definitions used in previous literature and to ensure articles that used 
different terms for their smoking groups were identified. Keywords for neurocognitive deficits were organised into themes which were created by reviewing articles such as Franken and van der Wettering (2015) and Volkow et al. (2010) for key words. The themes included: general cognition (a broad theme encompassing terms such as "neural correlate" and "neurocognitive mechanism"), impulsivity, control (e.g. cognitive control and performance monitoring), attention, motivation, memory, and craving. The strategy for searching each database was combining a list of key words relating to nicotine dependence with a list of key words relating to a theme of cognitive deficits. The boolean operator "AND" was used to ensure each paper contained at least one key word from each component. The list of key words used for the search process is included in Appendix A.

\subsubsection{Study selection}

Studies that were included in the review went through an initial three-stage process. First, a list was created in each database to find articles featuring a combination of the two types of key words outlined in the previous section. This was performed for each database and the list was reviewed to identify titles that related to the research question and eligibility criteria. Second, the abstract of each article that was retained based on the title was examined for whether it related to the research question and eligibility criteria. Articles that were inconsistent were discarded, articles that matched the research question were downloaded and stored in a reference manager. Finally, each article retained on the basis of its abstract was read in full and assessed for eligibility in the final review.

\subsubsection{Data collection process}

For each eligible study that was included in the final review, the method and results were recorded. The components of interest in the methods section were how the smoking groups were defined and identified, and what measures were used. Furthermore, the conclusions from each study were recorded to synthesise the results together in the review. 


\subsection{Results}

\subsubsection{Study selection}

There were 8663 (Medline), 6735 (Scopus), and 5184 (PsychInfo) records. The search criteria was designed to be inclusive due to the inconsistency in terminology, but a notable deviation in the search strategy was the general smoking terms such as nicotine dependence and substance use were removed. This was because it returned an unmanageable number of search results. Therefore, the search had a more specific focus on different terms for lighter and heavier smokers. After screening the titles and removing duplicates across the three databases, this left 180 articles. After screening the abstracts, 96 articles were read in full to assess if they met the inclusion criteria. In the process of reading these articles, an additional two studies were identified from the reference list that could be eligible to leave 98 articles. A total of 42 articles fulfilled the inclusion criteria after reading the full texts.

Reasons for excluding the articles were: the study only included daily or dependent smokers ( $\mathrm{n}=21)$, the study did not use a cognitive task or investigate cognitive deficits $(n=18)$, the only analysis was a correlation of nicotine dependence with the main outcome $(n=9)$, there was no breakdown of smoking characteristics $(n=2)$, there was not a direct comparison between smoking groups $(\mathrm{n}=2)$, the study investigated the pharmacological effects of a drug on smokers $(n=1)$, high and low dependency was based on poly-substance use $(n=1)$, there were not two separate smoking groups $(n=1)$, and the sample included participants under the age of $18(n=1)$.

\subsubsection{Smoking labels and criteria}

Across the 42 studies included in the final review, there were 37 unique combinations of smoking labels and criteria. Table 2.1 provides the key demographic information, and Table 2.2 presents the labels and criteria used to define the smoking groups in each study. Both tables are presented at the end of the chapter to prevent disruption due to their length spanning several pages. Broken down into the separate components of labels and criteria, there were 17 unique combinations of smoking labels for two or more smoking groups. The most frequent labelling conventions were light and 
heavy smokers $(n=10)$, daily and non-daily smokers $(n=6)$, occasional and dependent smokers $(n=5)$, occasional and daily smokers $(n=3)$, low and high dependence smokers $(n=3)$, chippers and regular smokers $(n=2)$, non-dependent and dependent smokers $(\mathrm{n}=2)$, and weekly and daily smokers $(\mathrm{n}=2)$. The remaining studies had unique labelling conventions. The slightly unusual term chipper refers to nondependent smokers who smoke infrequently, originating from street slang for heroin users (Coggins et al. 2009).

For the criteria that determined which smoking group the participant was placed in, there was considerably more variability. There were 32 unique combinations of criteria that determined whether the participant was a lighter or heavier smoker. The most frequent criteria were differentiating between daily and non-daily smoking patterns $(n=7)$, smoking $<10$ or $>10$ cigarettes per day $(n=2),<20$ or $\geq 20$ cigarettes per day $(n=2),<10$ or $\geq 20$ cigarettes per day $(n=2)$, and $<3$ or $\geq 3$ on their FTCD score $(n=2)$. The remaining studies used unique ways of differentiating between lighter and heavier smokers.

\subsubsection{Narrative review}

In the pre-registration protocol for this systematic review, the plan was to conduct a narrative, qualitative review of the literature unless two or more similar studies were conducted. Some topics of research had two or more studies, but the systematic review remained a narrative review due to the large degree of heterogeneity in smoking labels and criteria. This means that within studies, the smoking groups were relatively lighter and heavier, but as indicated in Section 2.4.2, the heterogeneity in smoking definitions meant the groups were not necessarily comparable across studies. Therefore, there may have been several studies on the same topic, but the methodological differences would have made a meta-analysis misleading as a quantitative review (Siddaway, Wood, and Hedges, 2019). When describing individual studies, the labels used within each study are used to remain consistent with the author's terminology. However, for brevity, the smoking groups are described as lighter and heavier smokers when describing the results across multiple studies. This is to communicate the fact that the smokers consisted of two groups of relatively lighter and heavier smokers, but each study may not have used the same criteria to define them. 
The 42 articles spanned a range of topics relating to neurocognitive mechanisms. Similar to systematic reviews such as Luijten et al. (2014), the results are organised in themes. The themes are centred around the six brain networks implicated in addiction following the updated iRISA model (Zilverstand et al. 2018). However, the key difference in this review is the results focus on the difference between smoking groups, and encompasses both behavioural and imaging research. Each network is briefly outlined to describe the kind of behaviour it encompasses, before providing an overview of the findings from each study.

\subsubsection{Executive network}

This network underlies the selection of an appropriate behavioural response, and includes behavioural and cognitive inhibition, and cognitive flexibility. It is key for representing and maintaining goals in motivated behaviour. This network is consistent with inhibitory control which is involved with behaviours such as inhibition and error processing. Five studies examined behaviours relating to the executive network.

\section{Inhibitory control}

There were three studies which investigated inhibitory control using both behavioural and neuroimaging measures. Two studies focused on behavioural measures. Yakir et al. (2007) investigated a range of groups including non-smokers, past smokers, current smokers, and occasional smokers. Participants were an all female sample, and completed a battery of cognitive tests on attention, information processing, memory, working memory, and impulsivity. The only result to survive correcting for multiple comparisons across 16 tests was impulsivity, as measured by the matching familiar figures test. Participants must match figures to an exemplar, and a higher number of errors in matching the figures indicates greater impulsivity. Current and former smokers made significantly more commission errors than non-smokers. Occasional smokers made slightly more errors than non-smokers, but this test was not reported as part of the planned comparisons. Billieux et al. (2010) found that moderate smokers had worse inhibition capacity than light smokers. They used a go-stop task where participants had to inhibit a prepotent response. Light smokers had a lower stopsignal reaction time (SSRT) than moderate smokers. A regression analysis showed that inhibitory capacity predicted nicotine dependence after age, craving, and processing 
speed were controlled for. This suggests that moderate smokers had poorer inhibition capacity than light smokers.

There was one study that used neuroimaging measures. Brinkmeyer et al. (2011) studied P50 gating in a large cross-site sample of non-smokers, light smokers, and heavy smokers. Sensory gating is the ability to ignore repetitive stimuli and focus attention which is related to inhibitory control. Lower values indicate a deficit, as there is a smaller difference in response to repeated auditory tones. Heavy smokers had a significantly smaller difference in P50 activity than light smokers and non-smokers. Light smokers had a smaller difference than non-smokers, but the difference between the two groups was not significantly different. This means that heavy smokers displayed impaired inhibitory function through diminished P50 sensory gating.

Summary: Three studies included a measure of inhibitory control, but used different measures. Billieux et al. (2010) showed that moderate smokers had poorer response inhibition than light smokers. However, there was no control group to anchor the results. Brinkmeyer et al. (2011) and Yakir et al. (2007) included a non-smoking control group. Both studies found that their group of heavier smokers had poorer inhibitory control than non-smokers. The lighter smokers held an intermediary position in both studies, but neither reported that they were significantly different to non-smokers.

\section{Cognitive flexibility}

Nesic et al. (2011) investigated low and high dependent smokers on cognitive flexibility. Participants completed the intra-extra dimensional set-shift test (IED) which measures different facets of executive function such as inhibiting a habitual response and cognitive flexibility. Participants were further divided into groups of abstinent and satiated smokers. Satiated high dependent smokers made significantly more errors than abstinent high dependent smokers. There were no other significant differences between the groups. This shows that acute smoking decreased cognitive flexibility in high dependent smokers, but it did not have a significant impact on low dependent smokers.

Summary: Only one study investigated cognitive flexibility. Satiated high dependent 
smokers had worse cognitive flexibility than abstinent high dependent smokers. Further research is required to explore this finding, as it is not clear why abstinent high dependent smokers in the initial state of withdrawal would have greater cognitive flexibility than satiated high dependent smokers.

\section{Error processing}

Rass et al. (2014) investigated error monitoring in non-smokers, intermittent smokers, and daily smokers. Participants completed two tasks: the Eriksen Flanker and Go/No-Go task. The Eriksen Flanker task requires the participant to ignore peripheral distractors and identify a central cue. The Go/No-Go task requires participants to respond to one type of stimulus and inhibit a response to a less frequent stimulus. The ERN and Pe ERP components were measured as indicators of error monitoring when participants made mistakes. Intermittent smokers had greater Pe amplitude than daily smokers on the Go/No-Go task. Although intermittent smokers had greater Pe amplitude than non-smokers, the difference was not statistically significant. There were no significant effects for the Eriksen Flanker task. The Pe waveform is thought to represent conscious awareness of making a mistake (Luijten et al. 2014), therefore Rass et al. (2014) interpreted the results as intermittent smokers possessing certain characteristics which allows them to maintain control over their smoking behaviour.

Summary: Only one study studied error processing. Intermittent smokers had a significantly larger Pe amplitude than daily smokers, and a larger but not statistically significant Pe amplitude than non-smokers. Further research is required to explore this result. Rass et al. (2014) reported that they explored both inhibitory control and error processing, but only focused on the ERN and Pe in response to mistakes. Future research should focus on the widely researched N2 and P3 waveforms if they want to explore inhibitory control.

\subsubsection{Salience network}

The salience network responds to attention grabbing stimuli. It is involved in reallocating attention resources to salient stimuli. In drug use, this is often related to drugseeking behaviours, such as attending to drug-related cues. There were ten studies 
related to the salience network.

\section{Attentional bias}

There were six studies which explored attentional bias towards smoking cues. There was one observational study. Herman (1974) conducted one of the earliest known studies on light and heavy smokers. An elaborate procedure was created where participants completed a maze task at a desk. Some participants completed the task while a lamp illuminated a cup holding a cigarette in a high salience condition, while others completed the task without the lamp illuminating the cup in a low salience condition. After the maze task was completed, the time to smoking a cigarette and the number of cigarettes smoked was recorded in a 30 minute observation period. Heavy smokers had a small, non-significant difference in time until the first cigarette and number of cigarettes smoked. However, light smokers decreased the latency to smoking and increased the number of cigarettes smoked in the high salience condition. This shows that the salience of smoking cues can influence the smoking behaviour of light smokers, whereas heavy smokers continue at a similar rate.

There were four studies that used a visual probe task to measure attentional bias. Hogarth et al. (2003) conducted two studies where they compared non-smokers, light smokers, and heavy smokers on attentional bias towards smoking cues. Participants completed a visual probe task where they responded to a probe replacing a smoking or non-smoking image. Attentional bias to smoking cues is indicated by faster responses to probes replacing smoking images. In study one, light smokers showed the greatest attentional bias to smoking cues, but it was not significant using a traditional alpha level of .05. In experiment two with a larger sample size, light smokers had greater attentional bias to smoking cues than heavy smokers and non-smokers. There was not a significant difference between heavy smokers and non-smokers. This suggests that light smokers find smoking cues salient, and they attract their attention allowing them to respond faster. Mogg, Field, and Bradley (2005) compared low and moderate nicotine dependent smokers on their attentional bias towards smoking cues using a visual probe task and eye-tracking. The total gaze dwell time on smoking and non-smoking cues was measured. The low dependence group spent more time looking at the smoking images than moderate dependent smokers. However, there was not a significant 
effect when response times were used as the outcome measure. This study shows that low dependence smokers display greater attentional bias towards smoking cues, at least when using direct eye-tracking measures. Vollstädt-Klein et al. (2011) further investigated attentional bias in non-smokers, and different groups of light and heavy smokers. Participants completed a visual probe task with smoking and non-smokers images. The smoking groups were categorised in different ways to explore how robust the conclusions were. This included FTCD score, number of cigarettes smoked per day, and exhaled $\mathrm{CO}$ concentration. The heavier smoking group displayed significantly greater attentional bias towards smoking cues than non-smokers for all three ways of creating the smoking groups. Heavier smokers also had greater attentional bias than lighter smokers when categorised using the CO and FTCD, but not by the number of cigarettes per day. This comparison did not survive correcting for multiple comparisons. This shows that heavier smokers showed greater attentional bias, and this effect was robust to different ways of classifying smokers. Dickter and Forestell (2012) compared occasional and daily smokers on attentional bias towards active (human smokers) and inactive (smoking objects) smoking cues. They were also interested in comparing smokers who had a smoking parent to those who did not. There was no significant effect in occasional smokers. In daily smokers, those with a smoking parent showed greater attentional bias towards inactive smoking cues than those without a smoking parent. The effect was not significant for active smoking cues. This shows that for daily smokers from a parental smoking background, inactive smoking cues are salient.

There was one study that used a rapid serial visual presentation task. Brown, Forster, and Duka (2018) investigated attentional bias by manipulating whether distractors were task relevant or irrelevant. If smoking cues used as distractors are salient, they should grab attention regardless of whether they are task relevant or irrelevant. Top-down goals had a large influence on involuntary attention as distractors only had an effect when they were task relevant. There was no effect of smoking group (supported by Bayesian analyses), suggesting none of the groups found smoking stimuli more salient than neutral stimuli.

Summary: Three studies investigated attentional bias between lighter and heavier 
smokers using a visual probe task with smoking and neutral images. This produced conflicting results as Hogarth et al. (2003) and Mogg et al. (2005) found that lighter smokers displayed greater attentional bias to smoking cues than heavier smokers, but Vollstädt-Klein et al. (2011) found that heavier smokers displayed greater attentional bias than lighter smokers. Three studies explored other factors that may be involved in attentional bias. Herman (1974) conducted the earliest known study on salience and smoking behaviour. When smoking cues were salient in the environment, light smokers increased their smoking behaviour to similar levels as heavy smokers. Dickter and Forestell (2012) showed that only daily smokers with smoking parents showed attentional bias towards inactive smoking cues. However, caution should be exercised when interpreting these results due to the small sample sizes of each sub-group. The visual probe task allows participants to freely explore the images before a probe appears. Therefore, Brown et al. (2018) manipulated the task instructions to explore whether top-down goals would affect attentional bias to smoking cues. Only task relevant distractors affected cues, and this was consistent across all three groups. This suggests that smoking cues were not particularly salient for either smoking group in comparison to neutral cues. Due to the conflict in research on attentional bias, it is clear that further research is required to understand the subtleties in the design and findings of such studies.

\section{Startle response}

Cui et al. (2012) used three different groups of treatment seeking smokers to investigate startle cue reactivity. This task requires the participants to watch a series of cigarette-related, neutral, pleasant, and unpleasant images. On a small number of trials, an infrequent loud burst of sound is played through the participant's headphones. A smaller startle response indicates an image condition is salient, or more motivationally relevant. Relative to neutral images, heavy smokers showed the greatest startle attenuation to cigarette-related images. Light smokers had the smallest attenuation and moderate smokers held an intermediary position. Heavy smokers had a significantly higher startle attenuation than light smokers, but no other comparison survived correcting for multiple comparisons. This suggests that cigarette-related stimuli are more salient for heavy smokers than light smokers, as the stimuli produced a smaller startle 
response.

Summary: Using a different measure of salience to attentional bias studies, Cue et al. (2012) found that smoking cues are salient to heavy smokers in comparison to light smokers. This pattern of results supports the findings of Vollstädt-Klein et al. (2011) and Dickter and Forestell (2012) using the visual probe task as an alternate measure of the salience of smoking cues.

\section{Graphic warning labels and plain packaging}

Three recent studies explored how lighter and heavier smokers respond to graphic warning labels. In many countries, tobacco control policies enforce cigarette packaging to feature health warnings (Moodie et al. 2018). This ranges from small warnings on traditional branded packaging, to plain packaging with large warnings. Cochran et al. (2017) investigated the effect of graphic warning labels on cigarette packaging. They were interested in whether disgust or anxiety based warnings would affect motivated attention, measured using ERPs. Low nicotine dependence smokers had lower P3 amplitudes at the electrode site $\mathrm{Fz}$, but high nicotine dependence smokers had lower P3 amplitude at sites Cz and Pz. Increased P3 amplitude is thought to represent cue processing which requires more cognitive resources. These results tentatively suggest that more dependent smokers are habituated to smoking stimuli and the responses to the packaging are more automatic. In contrast, less dependent smokers find the stimuli more novel, and allocate greater attentional resources. Maynard et al. (2017) measured visual attention and its neural correlates to plain and branded cigarette packaging. The sample included non-smokers, weekly smokers, and daily smokers. Participants had their eye-movements measured and neural responses measured using fMRI to each stimulus. Daily smokers made significantly more fixations to the warnings on plain packs than branded packs. There were no significant effects in weekly smokers and non-smokers. For the imaging data, there was a significant effect in the right amygdala. Warning labels on plain packaging decreased activity in weekly smokers, but increased activity in daily smokers. The tentative interpretation is that branded packs create a positive or cue-induced response in weekly smokers. Gantiva et al. (2019) compared non-smokers, weekly, and daily smokers on their physiological 
response to cigarette packaging without graphic warning labels, and packaging with different amounts of label coverage. Across all groups, graphic warning labels that covered $60 \%$ of the pack produced a higher skin conductance response, corrugator muscle activity, and startle response than packaging without graphic warning labels. However, there was no significant interaction with smoking group in any outcome variable. This suggests that regardless of smoking group, cigarette packaging with large graphic warning labels produces a greater arousal response.

Summary: Three studies investigated the responses of lighter and heaviers smokers to graphic warning labels or combining them with plain cigarette packaging. Gantiva et al. (2019) did not find that smoking group moderated the physiological responses to graphic warning labels. Across all participants, larger warning labels produced higher responses. The remaining two studies used different imaging methods and found complicated, but potentially complementary results. Cochran et al. (2017) focused on the P3 component using EEG, and concluded that cigarette packaging containing graphic warning labels was more eye-catching through engaging attentional resources for low nicotine dependence smokers. On the other hand, high nicotine dependence smokers showed more of an attenuated attentional response, potentially as they are habituated to the sight of cigarette packaging. Maynard et al. (2017) found using fMRI that there was decreased activity in the right amygdala for plain packaging with warning labels for weekly smokers. They concluded that decreased activity could represent a cue-induced response in weekly smokers. This finding is potentially complementary to Cochran et al. (2017) as they concluded the warning labels require greater attentional resources. Amygdala activity cannot be detected using EEG, but the pattern of results suggest lighter smokers require more attentional resources when looking at graphic warning labels on cigarette packaging, as the response is not as automatic as heavier smokers.

\subsubsection{Reward network}

This network is involved in appraising the motivational value of stimuli, and reward expectations. For example, attributing greater value to a particular type of stimulus, or delay discounting, where short-term rewards are valued over long-term losses. This 
network was the most popular area of interest with 16 studies focusing on different behaviours.

\section{Reward processing}

Six studies investigated reward processing (or how groups evaluate the value of particular stimuli). This included three studies that evaluated the choice of rewards in lighter and heavier smoker. Bühler et al. (2010) investigated reward processing in occasional and dependent smokers. They looked at the physical effort participants would use to try and get monetary or cigarette rewards. Occasional smokers exerted more effort for monetary rewards, but there was not a significant difference between the rewards in dependent smokers. This pattern of results was also reflected in fMRI recordings, where there was greater activity in the mesocorticolimibic system in occasional smokers for monetary rewards relative to drug rewards. This suggests that non-drug rewards have decreased value in dependent smokers. Lawn et al. (2015) developed a novel task to investigate reward processing in dependent and occasional smokers. The task measured the relative preference for different rewards (chocolate, music, paper, or cigarettes) and the effort used to obtain the reward. In each trial, participants chose between two rewards, one of which indicated a larger reward and the other a smaller reward. Dependent smokers chose cigarettes more and chocolate less than occasional smokers. Within the groups, dependent smokers chose cigarettes more than chocolate and music, and occasional smokers chose chocolate more than cigarettes. For the effort used to obtain the rewards, dependent smokers exerted more effort for cigarettes than occasional smokers. However, within the groups, there were no significant differences within dependent smokers, but occasional smokers worked harder for chocolate rewards than cigarette rewards. This study suggests that rather than non-drug rewards being devalued, nicotine dependence is characterised by an increased sensitivity for drug-related rewards. Non-drug rewards were relatively similar for both groups. Lawn et al. (2018) investigated dependent and occasional smokers on reward choices, but they also explored whether Pramipexole, a dopamine agonist, could affect motivation. At a relatively low dose, it has an inhibitory effect on phasic dopamine firing, with the aim of deceasing motivation for cigarettes. Similar to Lawn et al. (2015), dependent smokers chose cigarettes more and chocolate less than 
occasional smokers. Dependent smokers also exerted more effort for cigarettes than occasional smokers. Supported by Bayesian analyses, Pramipexole did not affect reward choice or button pressing in either smoking group. Participants also completed a cigarette purchasing task, where dependent smokers consistently scored higher than occasional smokers on all measures including the amount of cigarettes they would smoke at zero cost. These results support Lawn et al. (2015) where dependent smokers have an increased sensitivity to drug-related rewards. However, an acute dose of Pramipexole did not reduce motivation for cigarettes in either smoking group.

Three studies focused on how reward processing could be manipulated. Venugopalan et al. (2011) studied low frequency and high-frequency smokers. Low-frequency smokers were further split into those that had smoked for less than a year (early), and those who had smoked for more than three years (stable). Participants performed a cigarette self-administration task where participants could exert effort to earn cigarettes on a progressive reward schedule. The authors were further interested in how depleting dopamine (through reducing thenylaline and tyrosine) would affect motivation to earn cigarettes. High-frequency smokers worked harder to earn nicotine cigarettes than both low-frequency groups. However, depleting dopamine reduced the breakpoint in all smokers. This suggests that reducing dopamine does not differentially affect particular types of smokers, but that dopamine is related to motivation for cigarette rewards in general. Hogarth and Chase (2012) conducted two studies where they compared daily and non-daily smokers. They used a concurrent choice procedure where participants could win cigarettes or chocolate. In study one, participants were trained to recognise stimuli that indicated a tobacco reward, and then tried to extinguish the relationship. This was to test whether dependence would impact a learnt relationship being extinguished. This rate of extinction did not differ by smoking group, as all participants decreased responding to stimuli that were no longer rewarded. In study two, the process was repeated, but the reinforcement rate was steadily reduced. Both daily and non-daily smokers showed an increase in tobacco choice as the reinforcement rate decreased. Importantly for reward processing, daily smokers chose the tobacco reward over the chocolate reward more than non-daily smokers, suggesting there was an oversensitivity to drug rewards in daily smokers. 
Hogarth, Chase, and Baess (2012) investigated goal-directed control in daily and nondaily smokers. Participants completed concurrent choice training where they learnt two instrumental responses to win water or chocolate. Participants were then assigned to consume one of the rewards until they were satiated. An extinction test was then performed where participants were not informed whether they won more water or chocolate until the end of the trials. All participants reduced responding to the devalued outcome in the extinction test, and there was no significant difference between the two smoking groups in their reduction. This suggests that there is not a larger difference between daily and non-daily smokers in goal-directed control to non-drug rewards.

Summary: Two studies showed that dependent smokers would choose cigarettes over chocolate rewards, and work harder to receive cigarettes than occasional smokers (Lawn et al. 2015; 2018). Hogarth and Chase (2012) showed that daily smokers would choose a cigarette reward over a chocolate reward more than non-daily smokers. Venugopalan et al. (2011) found that high-frequency smokers would work harder for cigarette rewards than both low-frequency smoking groups. This shows converging evidence that heavier smokers are overly sensitive to cigarette rewards in comparison to lighter smokers, supporting a common view of addictive behaviour suggesting that dependence is characterised by a blunted response to non-drug rewards (Zilverstand et al. 2018). Bühler et al. (2010) showed that occasional smokers would work harder for monetary rewards, whereas dependent smokers had a smaller difference in their response to cigarettes and money. This shows that non-drug rewards are less attractive for dependent smokers. However, the limitation of this study is money could easily be used to buy cigarettes, so it may not reflect a true non-drug stimulus. Hogarth et al. (2012) demonstrated that both daily and non-daily smokers exhibit goal-directed learning, and show a similar devaluation in non-drug rewards when satiated. Therefore, there is more consistent evidence that heavier smokers are characterised by a hypersensitivity to drug-related rewards in comparison to lighter smokers, rather than hyposensitivity to non-drug rewards. 


\section{Delay discounting}

Eight studies investigated delay discounting. Heyman and Gibb (2006) compared non-smokers, chippers, and regular smokers. They used two different versions of the delay discounting task: one version offered hypothetical rewards and the other offered actual rewards. Delay discounting tasks present the participant with different amounts of money which they can choose immediately, or they can choose a different amount of money after a delay. For actual rewards, regular smokers had significantly higher discounting rates than chippers and non-smokers, indicating that they preferred smaller short-term rewards over larger long-term rewards. The pairwise comparison between chippers and non-smokers was not reported. However, when hypothetical rewards were used, there were no significant differences across the groups. A limitation of their procedure was there was no counterbalancing, so participants always completed the hypothetical task first, and then actual rewards. This may have affected their pattern of responding if they knew they would win money in the second task. Johnson, Bickel, and Baker (2007) compared delay discounting to different outcomes in non-smokers, light smokers, and heavy smokers. For hypothetical money rewards, light and heavy smokers discounted rewards more than nonsmokers, but did not differ significantly between themselves with a small effect size. However, there was not a significant effect across groups when other outcomes were used, such as health discounting. This was also the case for both smoking groups when cigarette outcomes were discounted. Sweitzer et al. (2008) compared never smokers, non-smokers who had tried the occasional cigarette, ex-smokers, and current smokers. Current smokers were classified into lighter and heavier smokers in two different ways. The first through the number of cigarettes they smoked per day to create groups of light, moderate, and heavy smokers. The second through their FTCD scores to create groups of low and high dependence. When smokers were classified through the number of cigarettes per day, current smokers had higher discounting rates than never smokers, tried-it smokers, and ex-smokers. However, there was no significant effect across the three groups. When smokers were classified into low and high dependence, high dependence smokers had a higher discounting rate than low dependence smokers. Low dependence smokers were more similar to the other three groups with smaller discounting rates. This suggests that dependence may be 
more important than simply frequency of use in affecting discounting rates. Rezvanfard et al. (2010) used a sample of male medical students to complete a battery of neuropsychological tests. Participants were separated into non-smokers, lightly dependent smokers, and heavily dependent smokers. Part of the battery was a Persian version of the delay discounting task. Heavily dependent smokers had a higher discounting rate than lightly dependent smokers and non-smokers. Light dependent smokers and non-smokers scored similarly and did not differ significantly. Stillwell and Tunney (2011) compared a large sample of non-smokers, non-daily smokers, and daily smokers. Participants completed an online delay discounting task for delayed or immediate monetary rewards. The study included a range of participants from different countries and matched the rewards to their own currency. Daily smokers had a higher discounting rate than non-daily smokers, who in turn had a higher discounting rate than non-smokers. This suggests that there was a dose-response relationship with more frequent smokers having larger discounting rates and preferred sooner smaller rewards than non-daily smokers and non-smokers. Poltavski and Weatherly (2013) investigated probability and delay discounting in non-smokers, light, and heavy smokers. Similar to Johnson et al. (2007), participants were presented with a range of commodities to discount. Probability discounting refers to the choice between a large probabilistic reward and a smaller definite reward. In contrast to previous studies, there was no significant interaction between smoking group and type of commodity for delay discounting. However, across all commodities, heavy smokers had a shallower rate of discounting than non-smokers for probability discounting. Light smokers were at an intermediate level between heavy smokers and non-smokers, but did not differ significantly from either. Carim-Todd, Mitchell, and Oken (2016) compared chippers, heavy smokers, and non-smokers on a range of impulsivity tasks. Participants completed a delay discounting task and a flanker task to measure response inhibition. Both smoking groups were more impulsive than non-smokers, as they tended to prioritise short-term gains. However, there was not a significant difference between the smoking groups and the pattern of results was similar. There was no significant difference between any group on the flanker task. This study is also relevant to the executive network as the flanker task measures cognitive control (similar to Rass et al. 2014), but it was included in this section due to Carim-Todd et al. (2016) 
focusing on the delay discounting results. This shows that chippers and heavy smokers had a higher degree of discounting than non-smokers. Rass, Ahn, and O'Donnell (2016) compared non-smokers, non-daily smokers, and daily smokers on delay discounting and resting state EEG. Participants completed a standard delay discounting task. Daily and non-daily smokers had higher discounting rates than non-smokers for rewards at a 3 and 10 year delay. The pairwise comparison between each smoking group was not reported. However, the result for a 3 year delay should be interpreted with caution as the ANOVA was non-significant at the traditional alpha level of .05. This means there is tentative evidence suggesting daily and non-daily smokers show greater delay discounting than non-smokers.

Summary: The eight studies that investigated delay discounting produced largely consistent results for heavier smokers having a higher discounting rate than nonsmokers. Poltavski and Weatherly (2013) found similar responding for delay discounting across all groups, but did find that heavy smokers displayed shallower probability discounting than non-smokers. The remaining studies consistently showed that heavier smokers prioritised short-term rewards over long-term gains more than nonsmokers. However, the position of lighter smokers was more variable. Across the studies, four found they were similar to heavier smokers (Johnson et al. 2007; Sweitzer et al. 2008; Rass et al. 2016; Carim-Todd et al. 2016), one found they held an intermediary position (Stillwell and Tunney 2011), and two found they were similar to nonsmokers (Heyman and Gibb 2006; Rezvanfard et al. 2010). This is where the variability in smoking criteria makes it difficult to draw conclusions on mixed results when similar tasks are used. Studies such as Sweitzer et al. (2008) demonstrated that the results were sensitive to the criteria used, as there was an effect when smokers were divided using FTCD scores, but not on cigarettes smoked per day. Therefore, it is not clear whether the heterogeneity in findings is due to the differences in criteria used to define the smoking groups.

\section{Craving}

There were two studies that explored craving using cognitive measures. Knott et al. (2008) investigated craving-induced EEG indices in regular smokers and chippers. 
Resting-state EEG was measured while participants held a control object and a lit cigarette. EEG measurements were taken while the participants had their eyes closed over left and right frontal sites. Alpha one and alpha two frequency bands were measured and a ratio of left to right asymmetry was calculated. The two smoking groups were further separated into males and females. Female regular smokers had greater alpha one and alpha two ratio than male regular smokers and female chippers. This indicates that regular female smokers had relatively lower activity in the left frontal area. This is thought to represent craving as a negative state, with relatively less alpha activation being related to deficits in the approach system and reward-directed responding (Knott et al. 2008). However, caution should be exercised when interpreting the results as there was a maximum group size of six. This means it was only sensitive enough to detect implausibly large effects. The findings would need to be confirmed in a larger sample. Piasecki et al. (2017) investigated cue reactivity to smoking images in occasional and daily smokers. Participants completed a pictureviewing oddball task while the P3 ERP component was measured. Three stimulus types were used: neutral, smoking, and drinking straws (as more of an active control to look like cigarettes but without the nicotine connotations). There was greater P3 amplitude for smoking and straw stimuli in comparison to neutral stimuli. P3 activity was smaller for smoking stimuli than straws. However, there was no interaction effect between stimuli and smoking group. In another analysis, participants with higher P3 amplitude to smoking and straw stimuli had higher post-test craving. This suggests that if there is a difference between smoker's responses to smoking stimuli, this study was not sensitive enough to detect it.

Summary: Two studies explored craving related behaviours in lighter and heavier smokers. However, there was no common task used for the studies to be comparable. Knott et al. (2008) explored craving-induced EEG, but the design and sample size only made it sensitive enough to detect implausibly large effect sizes. Piasecki et al. (2017) found no significant effects across the smoking groups for cue reactivity. 


\subsubsection{Habit network}

The habit network is involved in the automatisation of behaviour or habit learning. The shift to addictive behaviour is characterised by changing from goal-directed behaviour to compulsive drug-seeking (Everitt and Robbins 2016). There were three studies which investigated behaviours associated with the habit network.

Sayette et al. (2001) compared tobacco chippers and heavy smokers on a range of craving and behavioural measures. Participants were further divided into minimallydeprived and deprived groups to investigate the role of nicotine deprivation. There were two tasks of interest to this review: a response time task to auditory tones before and after cue exposure, and a behavioural choice task which measured the amount of money it would take to not smoke for an additional five minutes. Response time to identify a tone increased in all participants during the smoking condition where participants held a cigarette in comparison to the control condition where they held a roll of tape. However, there were no significant differences between the smoking groups. Relevant to the reward network, heavy smokers required more money to delay smoking than chippers. Deprivation also had an effect where deprived smokers required more money than minimally deprived smokers. These tasks show that both smoking groups find it distracting to hold a cigarette while completing a response task. Heavy smokers valued cigarettes more than chippers, and would require more money to delay smoking. Field, Mogg, and Bradley (2006) compared infrequent and frequent smokers on their ability to respond to auditory probes while they they were smoking, pseudo-smoking, or not smoking. Participants had to listen out for a specific tone in a dual-task procedure. In comparison to the non-smoking baseline, infrequent smokers were significantly slower to respond to the tone when they were smoking, but there was no significant difference in frequent smokers. This suggests that smoking is not an automatic behaviour for infrequent smokers, and it interferes with completing another task. Both infrequent and frequent smokers were slower in the pseudo-smoking condition than non-smokers. Motschman and Tiffany (2016) investigated automatic processes in occasional and heavy smokers. In a similar task to Field et al. (2006), 
participants completed a dual-task procedure where they had to identify a particular auditory probe while smoking, pretending to smoke, or not smoking. The results replicated Field et al. (2006) as occasional smokers were significantly slower to respond to tones when smoking and pretending to smoke. Heavy smokers were slower when pretending to smoke, but there was not a significant difference when they were actually smoking. This provides further support that smoking is not an automatic process in occasional smokers.

Summary: Three similar studies were performed on the automatisation of smoking behaviour. Sayette et al. (2001) found that response times to respond to a tone increased when holding a cigarette over a neutral stimulus in both chippers and heavy smokers. However, the two other studies found more consistent results where lighter smokers produced longer response times to tone while they were holding a cigarette in comparison to a neutral stimulus (Field et al. 2006; Motschman and Tiffany 2016). There was no significant difference in heavier smokers. This shows that smoking behaviour is not automatic in lighter smokers, and requires cognitive resources which slows down performance on an unrelated task.

\subsubsection{Self-directed network}

The self-directed network is associated with higher cognitive regulatory functions. For example, self-awareness and self-reflection. There were two studies that explored higher cognitive functions through tasks measuring implicit affective responses and implicit attitudes.

Haight, Dickter, and Forestell (2012) compared daily and occasional smokers in their implicit affective responses to smoking and non-smoking cues. Participants indicated whether a Chinese pictograph was pleasant or unpleasant after a smoking or nonsmoking cue was used as a prime. Daily smokers responded with a significantly greater proportion of pleasant responses to smoking cues than non-smoking cues. Conversely, the effect flipped for occasional smokers as they had a greater number of pleasant responses to non-smoking cues, but there was not a significant difference 
between the two cue types. This shows that daily smokers held a positive affective attitude towards smoking cues. Wardell et al. (2018) compared non-smokers, non-daily, and daily smokers on their implicit attitudes towards smoking and alcohol cues. Participants completed two implicit association tests: one for smoking words and one for alcohol words. Participants had to categorise alcohol stimuli as active or passive, and smoking stimuli as positive or negative. Smokers and non-smokers did not differ significantly on implicit alcohol arousal, but non-daily smokers had stronger implicit alcohol associations than daily smokers. Each group displayed a stronger negative bias towards smoking cues in comparison to sweets. Non-smokers had significantly stronger negative associations towards smoking cues than smokers, but the two smoking groups were not significantly different.

Summary: Two studies investigated implicit attitudes towards smoking cues with each study using different measures. Haight et al. (2012) used a priming approach where cues were briefly flashed before a neutral stimulus, and Wardell et al. (2018) used an implicit association test where participants had to categorise stimuli into different categories. These methodological differences make it difficult to interpret the results as Haight et al. (2012) found that daily smokers responded with a greater proportion of pleasant responses to smoking cues. On the other hand, Wardell et al. (2018) found that non-smokers had the strongest negative bias than non-daily and daily smokers. The two smoking groups did not differ significantly.

\subsubsection{Memory network}

The memory network is involved in associative learning of multiple cues and the flexible use of learned information. There were five studies that investigated the role of memory or learning.

Stevens (1976) performed an early study comparing non-smokers, light smokers, and heavy smokers on several complex learning tasks. These included the Hunter-Pascal Concept task, the Wisconsin Card Sorting task, the Standard Anagram task, and the Word-in-Context test. Heavy smokers performed worse than light smokers and nonsmokers on all tasks but the Word-in-Context test. Non-smokers and light smokers 
did not differ significantly between themselves. This suggests that on several facets of learning, heavy smokers perform worse than light smokers and non-smokers. Tait et al. (2000) compared non-smokers, light smokers, and heavy smokers on visual memory using the Sternberg task. Participants had to indicate whether a probe was from a positive or negative set of two, four, or six digit strings. Smoking participants completed the task under both deprived or minimally deprived conditions. Decision time increased with increasing set sizes, but there was not a significant effect of smoking group. Heavy smokers performed worse than non-smokers after 12 hour deprivation, but the effect was smaller and not significant when they were minimally deprived. These results suggest that smokers may not differ substantially from non-smokers on visual memory. However, deficits in heavy smokers may be explained by being in a state of nicotine deprivation. Fried, Watkinson, and Gray (2006) compared light and heavy smokers to non-smokers and former smokers in their general neurocognitive ability. The study used a prospective design where participants completed the battery between the ages of 9-12 prior to smoking, and again in late adolescence. When taking pre-smoking scores into account, heavy smokers performed worse than non-smoking controls on verbal IQ, vocabulary, and the Wechsler Adult Intelligence Test (WAIS). Light smokers performed worse than non-smoking controls on vocabulary, auditory recognition, full scale IQ, and WAIS information and arithmetic subtests. The results followed a dose-response relationship with heavy smokers performing worse than light smokers, who performed worse than non-smokers. However, the comparisons between light and heavy smokers were not reported. These results demonstrate the negative effect of heavy smoking on neurocognitive ability, mainly centring on verbal skills and intelligence. Harmsen, Bischof, and Brooks (2006) compared nicotine dependent and non-dependent smokers using the gambling task card game. Participants had to select cards from different decks and accumulate as much fictitious money as possible. Some decks were more likely to return long-term gains, but the participants had to learn this as they made their way through the decks, keeping track of their losses and gains. Lower scores indicated that participants did not adapt their behaviour and minimise losses. Dependent smokers scored slightly worse than nondependent smokers on the total score, but this difference was not statistically significant. Paelecke-Habermann et al. (2013) compared non-smokers, occasional smokers, 
and dependent smokers on implicit and explicit reward learning. To test implicit reward learning, participants completed an ice-cream-seller task with two conditions. Participants had to recognise which cues were associated with different preferences. One condition provided implicit feedback and the other was an explicit observation condition. To test explicit reward learning, participants completed a card-playing task. Participants were presented with different cards, and they had to learn through trial and error which numbers were a target or non-target. Non-smokers were significantly more successful than each smoking group in implicit reward learning when they were provided feedback, but both smoking groups did not differ significantly between conditions. Dependent smokers and occasional smokers made more omission errors than non-smokers on the explicit learning task. However, caution should be exercised in interpreting these results as the pairwise comparisons were based on a non-significant ANOVA. These results suggest that both occasional and dependent smokers have deficits in reward learning in comparison to non-smokers, particularly in implicit reward learning.

Summary: Two studies were also relevant to the reward network, but they are included here as they centre on reward learning, rather than simply choosing rewards. Harmsen et al. (2006) found that dependent smokers performed worse than nondependent smokers, but the difference was not statistically significant. This result may be explained by the findings of Paelecke-Habermann et al. (2013) who included a nonsmoking control group. There was not a significant difference between occasional and dependent smokers, but both smoking groups performed worse than non-smokers. This suggests that different types of smokers may have deficits in reward learning, but it may only be observed when anchored with a non-smoking control. The remaining three studies were more heterogeneous, and explored different facets of memory and learning. Stevens (1976) found that heavy smokers performed worse on different learning tests. Tait et al. (2000) found that there were no substantial differences between the groups on visual memory, but heavy smokers displayed deficits while experiencing nicotine deprivation. Finally, Fried et al. (2006) performed a prospective study where heavy smokers mainly had deficits in verbal skills and intelligence. Although light smokers had deficits in several domains in comparison to non-smokers. 


\subsection{Discussion}

\subsubsection{Smoking labels and criteria}

One of the key findings of this systematic review was the large heterogeneity in labels and criteria used to classify people into groups of lighter and heavier smokers. This echoes the concerns of Husten (2009) who highlighted the range of terms and criteria used to define light and intermittent smokers. There were 17 unique combinations of smoking labels and 32 unique combinations of criteria used to categorise the smokers. This is problematic as it makes it all but impossible to meaningfully compare the findings of different studies as they are usually using two different ways of grouping the smokers. An extreme example included two articles from the same research group reporting slightly different criteria for the same participants (Rass et al. 2014; 2016). Both studies had identical demographic information, suggesting the same participants were used. Although this may be more of an issue with clarity in reporting than heterogeneity in criteria, as one of the studies may have explained the criteria used more comprehensively. Nevertheless, this demonstrates the nonchalance of reporting practices for different smoking groups.

There were also problems with a large amount of variability in how the same measures were used. There were nine unique ways of dividing participants using their average number of cigarettes per day, ranging from less than 10 and more than 10 , to smoking between 5 and 14 cigarette per day and 25 or more per day. There was also a broad use of the FTCD, with seven ways of splitting the participants. This included subtle differences such as one study using less than three and three or more, while another study used three or less and more than three. One reason for these subtle differences is several studies used a median split on their measure of choice to create two groups. Using the sample median is rarely a good choice as it can produce a new cutoff in each study, meaning the results across different studies are difficult to compare (MacCallum et al. 2002; Altman and Royston 2006). It is important that research uses criteria that are consistent across studies in order to meaningfully compare the results. The large heterogeneity, and often data driven way of defining the smoking groups, suggest there is not a strong enough theoretical backing to follow a consistent approach across studies. 
There are several potential solutions to this problem. The first option is studies could focus on using criteria that are consistent across different studies and stable in participants. Husten (2009) concluded that the most valid way of categorising smokers was using daily versus non-daily smoking patterns. In the studies included in this review, this distinction was the most popular with 7 of 42 studies using it, although they were not always referred to as daily and non-daily smokers. Daily and non-daily smoking patterns appear to be the most stable smoking behaviour, and is similar to the questions used in large population studies. However, it is unlikely that all researchers will converge on one way of categorising smokers as it has been over ten years since Husten (2009) originally commented on the variability in definitions used for light and intermittent smoking. The second option offers a more pragmatic approach as researchers can test how robust their conclusions are when different criteria are used. Two studies explored this approach in the current review. Vollstädt-Klein et al. (2011) used three different ways of categorising lighter and heavier smokers: using cigarettes per day, FTCD scores, and exhaled Carbon Monoxide. They found that the results were largely consistent regardless of the way the smokers were dichotomised. Conversely, Sweitzer et al. (2008) used two different ways and found conflicting results. There was no significant difference on delay discounting between smoking groups categorised using cigarettes per day, but high dependence smokers performed worse than low dependence smokers when using the FTCD. The benefits of this pragmatic option include the ability to illustrate whether results are sensitive to one method of dichotomising smokers out of several alternatives. This means researchers can use their preferred way of categorising smokers in the absence of a universally accepted theory, but the results should be compared to alternate methods of defining smokers to evaluate the consistency of the findings. The final option is analysing the results using continuous measures such as the number of cigarettes per day, instead of dichotomising the measures into distinct groups. In their recommendations against using a median split, MacCallum et al. (2002) argue that using regression to treat the variables as continuous avoids all the problems of dichotomisation. Although group membership can be successfully predicted using nicotine dependence measures, there is still unexplained variance within the groups (Shiffman et al. 2012b). The inclusion criteria for this systematic review was including studies that contained two or more groups of 
lighter and heavier smokers. This was included as many studies using a correlational approach compare smokers and non-smokers, and include a supplementary analysis to see whether there is a relationship between their outcome measure and a measure of nicotine dependence. However, these studies usually have high inclusion criteria, such as smoking ten or more cigarettes per day, meaning that they exclude non-daily smokers. Therefore, one approach could be to include both continuous and dichotomous variables to classify smokers into groups. This approach was used by Billieux et al. (2010), and may represent a way to test how robust the results are to different ways to treating nicotine dependence, similar to the second approach of testing how robust the conclusions are to different group classifications.

\subsubsection{Summary of findings}

The aim of this systematic review was to document previous research in daily and non-daily smokers. Unlike most systematic reviews (e.g., Smith et al. 2014), the focus was not on a particular measure or construct, but on the population of interest. This returned 42 studies on different aspects of neurocognitive functioning. These mechanisms are a popular area of study in addictive behaviours, with the aim of understanding how they contribute to problematic drug use and targeting them for interventions (Franken and van der Wetering 2015). The results were organised using the highly influential iRISA model, which focuses on dysfunction in the prefrontal cortex being behind a syndrome of maladaptive behaviours in addiction (Goldstein and Volkow 2002; 2011; Zilverstand et al. 2018). The iRISA model focuses on dependent drug users, and considers infrequent users as being in a state of transition. As lighter smokers can maintain an infrequent pattern of use long-term (Shiffman et al. 2012b; 2012c), but find it difficult to quit smoking long-term (Tindle and Shiffman 2011; Kotz et al. 2012; Herbec et al. 2014), this review documented what was known about neurocognitive mechanisms associated with addictive behaviour in daily and non-daily smokers. The results were organised using the six networks used in Zilverstand et al. (2018).

Five studies examined behaviours relating to the executive network. Three studies investigated inhibitory control using different measures. Heavier smokers had poorer inhibitory control than non-smokers (Yakir et al. 2007; Brinkmeyer et al. 2011). Lighter smokers held an intermediary position between the two. Billieux et al. (2010) did 
not include a non-smoking control group, and found that heavier smokers performed worse than lighter smokers. One study investigated cognitive flexibility, and found that satiated heavier smokers performed worse than abstinent heavier smokers (Nesic et al. 2011). One study investigated error processing, and found that heavier smokers had a deficit in error processing in comparison to lighter smokers (Rass et al. 2014). Carim-Todd et al. (2016) was placed in the reward network as the main focus of the article was delay discounting, but they also included the Flanker task. There were no significant differences between non-smokers, chippers, and heavy smokers, but this only focused on response time measures. No significant differences on response time measures were observed in Rass et al. (2014). Therefore, group differences may only be observable using ERPs. These studies suggest that across different areas of study, heavier smokers consistently show deficits in behaviours relating to the executive network. The position of lighter smokers is less consistent. In inhibitory control, there is almost a dose-response relationship with the deficits. However, in error processing, lighter smokers were more similar to non-smokers, and heavier smokers displayed the deficit.

Ten studies related to the salience network. There were six studies which focused on attentional bias. There were mixed results, as two studies found that lighter smokers displayed greater attentional bias to smoking cues than heavier smokers (Hogarth et al. 2003; Mogg et al. 2005), and one study found heavier smokers displayed greater attentional bias than lighter smokers (Vollstädt-Klein et al. 2011). Using different paradigms, one study found that increased salience to smoking cues increased the smoking behaviour of lighter smokers, but not heavier smokers (Herman 1974). Dickter and Forestell (2012) found that heavier smokers with smoking parents would show greater attentional bias to cues that only contained smoking paraphernalia. One study found that when there were top-down goals, no group found smoking cues particularly salient (Brown et al. 2018). There was one study which investigated the startle response and cue reactivity. Heavier smokers exhibited a smaller attenuation than lighter smokers, suggesting heavier smokers find smoking cues more salient than neutral cues (Cui et al. 2012). Three recent studies investigated graphic warning labels and plain cigarette packaging. One study did not find any evidence that physiological responses to graphic warning labels differed by smoking group (Gantiva et al. 2019). 
Two studies found potentially complementary results using different methods. Using EEG measures, lighter smokers showed signs of allocating greater attentional resources to graphic warning labels (Cochran et al. 2017). Using fMRI measures, lighter smokers had decreased right amygdala activity to warning labels on plain packaging, potentially representing a cue-induced response, or requiring greater attentional resources (Maynard et al. 2017). This is potentially due to lighter smokers having less exposure to cigarette packaging than heavier smokers. The results relating to the salience network were less consistent, with some studies showing that lighter smokers find smoking cues more salient than heavier smokers, whereas others find the opposite pattern.

Neurocognitive mechanisms associated with the reward network was the most widely researched domain with 16 studies. Five studies focused on reward processing. There was consistent evidence that heavier smokers are over-sensitive to drugrelated rewards. Smokers would choose cigarettes over rewards such as chocolate (Hogarth and Chase 2012; Lawn et al. 2015; 2018). One study found that heavier smokers showed a blunted response to non-drug related rewards, as lighter smokers would work harder for money (Bühler et al. 2010). However, the choice of money as a non-drug reward complicates the interpretation. Sayette et al. (2001) was presented in the habit network, but their results are applicable here too. Heavier smokers would require more money to delay smoking than lighter smokers, suggesting smoking, or avoiding abstaining from smoking, is valuable to them. These differences appear to be isolated to the context of drug and non-drug rewards, as heavier and lighter smokers do not appear to have any global deficits in goal-directed learning (Hogarth et al. 2012). The most widely researched area in lighter and heavier smokers was delay discounting with eight studies using largely the same measure. Poltavski and Weatherly (2013) found similar responding for delay discounting between lighter and heavier smokers, but heavier smokers displayed shallower probability discounting than nonsmokers. The remaining seven studies consistently showed that heavier smokers prioritised short-term rewards in comparison to non-smokers. However, the position of lighter smokers was less consistent. Across the seven studies, four found they were similar to heavier smokers (Johnson et al. 2007; Sweitzer et al. 2008; Rass et al. 2016; Carim-Todd et al. 2016), one found they held an intermediary position (Stillwell and 
Tunney 2011), and two found they were similar to non-smokers (Heyman and Gibb 2006; Rezvanfard et al. 2010). Two studies investigated craving, but focused on different tasks. One study found implausibly large effects with small group sizes using EEG measures (Knott et al. 2008), while Piasecki et al. (2017) found that lighter and heavier smokers were not significantly different on ERP components associated with cue reactivity.

Three studies focused on the habit network, using largely comparable methods. One study found that the response time to identify a tone was similar for heavier and lighter smokers while they held a cigarette (Sayette et al. 2001). Conversely, two studies found that lighter smokers would take longer to respond to a tone while they were holding a cigarette in comparison to heavier smokers (Field et al. 2006; Motschman and Tiffany 2016). This suggests that smoking behaviour in lighter smokers is not yet automatic, and it interferes with task performance.

Two studies focused on the self-directed network, with both studies focusing on implicit responses to smoking cues. One study found that heavier smokers would attribute greater pleasant responses to smoking cues than lighter smokers (Haight et al. 2012). The second study used a slightly different paradigm and found that nonsmokers had the greatest negative bias to smoking cues, but lighter and heavier smokers did not differ significantly from each other.

Finally, five studies focused on the memory network. Two studies focused on reward learning and found that lighter and heavier smokers did not differ significantly (Harmsen et al. 2006; Paelecke-Habermann et al. 2013). However, the interpretation of this is clarified by the addition of a non-smoking control group, as both smoking groups performed worse than non-smokers in Paelecke-Habermann et al. (2013). One study found than heavier smokers were largely worse than lighter smokers and nonsmokers on different complex learning tasks (Stevens 1976). One study found that smokers and non-smokers did not differ significantly on visual memory, but this may be affected by nicotine deprivation as 12 hour abstinence results in heavier smokers performing worse than non-smokers (Tait et al. 2000). The final study was the only example of a prospective design (Fried et al. 2006). Participants completed a battery of neurocognitive tests in childhood and again in late adolescence. Heavier smokers performed worse than non-smokers on different facets of intelligence, and lighter 
smokers performed worse than non-smokers on a smaller selection including facets of intelligence and auditory recognition.

\subsubsection{Integrative model}

The iRISA model (Goldstein and Volkow 2002; 2011; Zilverstand et al. 2018) has been one of the most influential theories for outlining how neurocognitive deficits are associated with problematic drug use. The main focus of the model is on drug users who have already reached a state of dependence. Dependence should be characterised by increased salience to drug-related stimuli and decreased control over drug use in comparison to non-dependent users (Goldstein and Volkow 2011). The aim of this systematic review was to collate research that had included both lighter and heavier smokers, to explore whether the literature supports the predictions of the iRISA model. There were two possible general patterns of results. If the degree of deficit is proportional to the level of dependence, there would be a dose-response like relationship as heavier smokers would have worse neurocognitive deficits than lighter smokers, who would in turn have worse deficits than non-smokers. Alternatively, as some lighter smokers can continue to smoke on an infrequent basis indefinitely (Shiffman et al. 2012b; 2012c), this would suggest they may possess a kind of protective characteristic that allows them to continue smoking infrequently and not transition into a state of dependence. In this scenario, lighter smokers would be expected to be similar to non-smokers, and heavier smokers would display worse deficits than lighter smokers and non-smokers.

Across the six areas, there was broadly consistent evidence that heavier smokers possessed greater deficits than non-smokers for those studies that included a nonsmoking control group. For example, heavier smokers had worse delay discounting than non-smokers, choosing short-term rewards over long-term gains (e.g., Heyman and Gibb 2006; Johnson et al. 2007), and heavier smokers had worse inhibitory control than non-smokers (Yakir et al. 2007; Brinkmeyer et al. 2011). This is in line with previous research that exclusively focused on dependent smokers and non-smokers. In some studies, the results were consistent with predictions that lighter smokers would be an intermediary between heavier smokers and non-smokers. Heavier smokers had 
worse inhibitory control than lighter smokers, who in turn had worse inhibitory control than non-smokers (Brinkmeyer at al. 2011). Heavier smokers worked harder or chose drug-related rewards more than lighter smokers (Venugopalan et al. 2011; Hogarth and Chase 2012; Lawn et al. 2015; 2018). This demonstrates that in areas typically associated with behavioural deficits associated with addictive behaviour, the results were in line with the predictions that heavier smokers would perform worse than lighter smokers.

On the other hand, the importance of focusing on different smoking groups was shown with the inconsistency of findings in other areas. In delay discounting, heavier smokers consistently performed worse than non-smokers, but lighter smokers were similar to heavier smokers (Johnson et al. 2007; Sweitzer et al. 2008; Rass et al. 2016; Carim-Todd et al. 2016), held an intermediary position (Stillwell and Tunney 2011), or were similar to lighter smokers (Heyman and Gibb 2006; Rezvanfard et al. 2010). Similarly, some research demonstrated that lighter smokers showed greater attentional bias or found smoking cues to be more salient than heavier smokers (Herman 1974; Hogarth et al. 2003; Mogg et al. 2005), while other research demonstrated that heavier smokers showed greater attentional bias than lighter smokers (Vollstädt-Klein et al. 2011; Cui et al. 2012). In reward learning, both lighter and heavier smokers showed deficits in comparison to non-smokers (Harmsen et al. 2006; Paelecke-Habermann et al. 2013). In the ability to complete a secondary task while smoking, lighter smokers performed worse than heavier smokers (Field et al. 2006; Motschman and Tiffany 2016), but this can be attributed to the lower frequency of smoking in lighter smokers meaning they have had less practice. Across the studies included in this systematic review, there was evidence that lighter smokers display deficits in neurocognitive mechanisms associated with addictive behaviour.

These results show the importance of including both lighter and heavier smokers in the sample. If the results focused on dependent users and non-users like previous reviews (e.g., Crunelle et al. 2012; Luijten et al. 2014), the results would be complementary with heavier smokers consistently displaying greater deficits than nonsmokers. However, when a group of lighter smokers are included, the results are less consistent with some studies finding them similar to heavier smokers, an intermediary, or similar to non-smokers. However, there is more evidence in favour of the first 
predicted pattern of results with lighter smokers either holding an intermediary position or being more similar to heavier smokers. There were fewer studies that reported lighter smokers performed similarly to non-smokers. This means there is evidence that deficits in neurocognitive mechanisms associated with addictive behaviour are present in lighter smokers. Although more research is required to investigate the precise position of lighter smokers in relation to heavier smokers and non-smokers, if these mechanisms are as important to maintaining addictive behaviour as the iRISA model predicts (Goldstein and Volkow 2002; 2011; Zilverstand et al. 2018), then these deficits may have a role in the addictive behaviour of lighter smokers. Lighter smokers find it almost as difficult to quit smoking long-term as heavier smokers (Tindle and Shiffman 2011; Kotz et al. 2012; Herbec et al. 2014). This means it is important to further explore deficits in neurocognitive mechanisms in lighter and heavier smokers.

In integrating the results of the studies presented in this systematic review, there are two related caveats to acknowledge. First, the review remained a narrative review to describe the results across studies as there was too much heterogeneity in the methods. Although it was sensible to not perform a meta-analysis, this meant that a "vote-counting" approach was taken (Siddaway et al. 2019), where the number and direction of significant and non-significant results was used to summarise the areas of research. Caution needs to be exercised as the presence or absence of a significant effect could be due to each study having enough statistical power to detect the effect size of interest or not. This means a difference in statistical significance may not represent substantive differences in findings, just a difference in the ability to detect an effect. Only four studies reported performing a power analysis, so for the remaining studies it cannot be ruled out that any non-significant effects were due to the study being simply underpowered, rather than represent effects too small to be practically interesting. In addition, only two studies appropriately tested for a null effect, with both studies used Bayesian statistics (Brown et al. 2018; Lawn et al. 2018). This means for studies concluding two groups were similar to each other, the majority of the studies in the review did not use the appropriate statistical methods. Second, the heterogeneity in methods that prevented a meta-analysis being performed could have contributed to the variation in results. The smoking groups used in each study were based on different labels and definitions, and the tasks they performed were different even within 
the groups of mechanisms. For example, three studies explored inhibitory control, but the three studies used a matching familiar figures test (Yakir et al. 2007), a go-stop task (Billieux et al. 2010), and an ERP measure of P50 gating (Brinkmeyer et al. 2011). This means there are different sources of variation within the studies that could explain the difference in results. In order to have more confidence in the consistency of findings, more methodologically similar studies are required, and in future, a meaningful meta-analysis could be performed.

\subsubsection{Limitations}

The first limitation of this systematic review is the dilemma of treating dependence on a continuum or as a dichotomy. The approach taken by this review was to focus on studies that included two or more smoking groups, which naturally excluded any studies that treated dependence on a continuum unless both strategies were reported. The reason for this was many studies that include dependence as a continuum when investigating neurocognitive measures use it as a supplementary analysis. For example, Goudriaan et al. (2010) studied cue reactivity and nicotine dependence using fMRI. However, despite comparing "high" and "low" dependence smokers after performing a median split on FTCD scores, the inclusion criterion was to smoke at least 15 cigarettes per day. This means there were two groups of daily smokers with relatively high rates of smoking in each group, and infrequent or non-daily smokers were excluded. Despite focusing on a dichotomy of smoking groups to specifically target studies including infrequent or non-daily smokers, there was a separate issue of studies using inconsistent labels and definitions for the smoking groups, even when the same measures were used. There were nine unique ways of creating the groups through different thresholds of cigarettes per day (e.g., less than 10 and between 1020 vs less than nine and more than nine) and seven unique ways of using the FTCD (e.g., three or less and more than three vs less than four and four or more). This demonstrates even when researchers used the same measures, they produced different group classifications. A large number of studies used a median split to create these dichotomies, so each study was prone to subtle differences in the range of scores in the sample. In order to mitigate this issue in future studies, there are different solutions available that were reviewed in Section 2.5.1. Studies can focus on consistent, 
theory-driven way of differentiating between smoking groups, test how robust the conclusions are to different criteria, or treat smoking behaviour as a continuum but ensuring non-daily smokers are included in the sample.

Despite focusing on neurocognitive mechanisms, very few studies used brain imaging methods such as EEG and fMRI. Previous reviews have focused on more narrow areas of research and methods, such as Luijten et al. (2014) who only included EEG and fMRI measures of inhibitory control and error processing. However, as previous reviews had not focused on neurocognitive mechanisms in lighter and heavier smokers, the inclusion criteria for the measures and methods was intentionally wide ranging in order to capture all the previous studies focusing on neurocognitive mechanisms in lighter and heavier smokers. The result of so few studies using imaging methods prevented the review from synthesising the results by the brain area associated with the deficits like previous systematic reviews (Luijten et al. 2014; Zilverstand et al. 2018). Therefore, the review cannot offer any concise summary of the pattern of deficits found in lighter and heavier smokers. Despite organising the results into themes based on the networks associated with the iRISA model, the conclusions are limited to the general pattern of behavioural results, rather than a concise synthesis of deficits in each network.

The studies presented in this review could not provide any insight into the causal relationship between deficits in neurocognitive mechanisms and problematic drug use as there was only one study which used a prospective design. Fried et al. (2006) measured the performance of 9-12 year olds on a battery of neurocognitive tests, and again in late adolescence after drug use had been initiated. Controlling for prior performance eliminated or reduced some relationships between heavy drug use and the cognitive outcome measures. This shows that some deficits were already evident before the onset of drug use, and subsequent smoking produced additive effects. Relatedly, there appears to be no longitudinal studies following how neurocognitive deficits develop in lighter and heavier smokers. To truly test whether neurocognitive deficits follow a dose-response relationship, it would be important to track performance as smokers transition from lighter to heavier smokers. The remaining studies in the review were cross-sectional and prevent any cause and effect conclusions. Therefore, it is not clear whether differential patterns of neurocognitive deficits lead to heavier 
smoking, or whether heavier smoking causes more severe neurocognitive deficits.

The reporting practices of studies could also be improved, especially when it comes to including a diverse range of smokers. Many studies did not include a breakdown of demographic information for each smoking group included in the study. Table 2.1 shows the demographic information for each study, and several studies have missing information. For example, some studies did not include the gender breakdown, number of cigarettes smoked per day, or a measure of nicotine dependence such as the FTCD. It was even rarer for a study to report a breakdown of ethnicities. This is surprising given that non-daily smoking is more prevalent in non-white smokers, and the health implications of smoking disproportionately affect non-white smokers (St. Helen et al. 2019). This shows that the level of reporting in studies focusing on smokers should be improved in order to get a better understanding of their particular characteristics and habits.

A notable deviation from the protocol for this systematic review was not performing a meta-analysis on any of the outcomes with two or more studies. This means that it remained a narrative review and described the pattern of results across studies. A narrative review is appropriate when studies have used heterogeneous methods (Siddaway et al. 2019). Although this would have been limited to a small number of measures, opting out of performing a meta-analysis was due to the large heterogeneity in smoking labels and criteria. With 32 unique combinations of criteria used to differentiate lighter and heavier smokers, there were rarely consecutive studies that used the same criteria. The aim of a meta-analysis is to synthesise the effect sizes of multiple studies (Borenstein et al. 2011). Even when a random effects model is used that does not assume one true effect size, it is usually focusing on one outcome using different measures, not focusing on different measures using different grouping criteria. This means the meta-analytic effect size would have had a large degree of heterogeneity, and it would have been misleading to interpret.

\subsubsection{Conclusion}

The aim of this systematic review was to document previous research on neurocognitive mechanisms associated with addictive behaviour in daily and non-daily smokers. Each of the six main networks associated with addictive behaviour (Zilverstand et al. 
2018) contained at least two studies, but some domains within the networks received more attention than others. For example, delay discounting received the most research attention with eight articles, while other areas such as the startle response or cognitive flexibility featured only one article. Each network would benefit from further research, but this would be beyond the scope of one thesis. Therefore, there is a choice of focusing on behaviours with fewer studies, or focusing on areas of controversy which have received conflicting or counterintuitive findings. This thesis will focus on some of these areas of controversy. Focusing on areas with more prior research is to ensure there is a sufficient background to inform the studies in this thesis, rather than explore areas with sparser literature.

Therefore, this thesis will focus on two components: attentional bias and cognitive control. These two topics were chosen as they represent the two opposing influences of drive and control mechanisms associated with addictive behaviour (Franken and van der Wetering 2015). Following the predictions of the iRISA model, addictive behaviour is characterised by an increase in the drive for drug seeking combined with the decreased ability to control drug use (Goldstein and Volkow 2002; 2011). There is a direct link between the two components (Marhe, Luijten, and Franken 2014) as attentional bias can affect cognitive control processes (Detandt, Bazan, Quertemont, and Verbanck 2017). Both components are popular areas of study in the area of addictive behaviours in general, but there is less research available and each component has an element of controversy to address in daily and non-daily smokers. In this systematic review, there were seven studies related to attentional bias or the salience of smoking cues. Some studies demonstrated that lighter smokers displayed greater attentional bias to smoking cues than heavier smokers, while others found heavier smokers displayed greater attentional bias than lighter smokers. Two studies using the visual probe task to investigate attentional bias will be presented in Chapter Three.

On the second components, there were four studies that explored cognitive control with three focusing on inhibitory control and one exploring error processing. There were three studies that explored inhibitory control in idiosyncratic ways, focusing on matching figures, a speeded response time task, and an EEG measure of sensory gating. There was only one study that explored error processing using EEG measures and a speeded response time task (Rass et al. 2014), and they found counterintuitive 
findings with non-daily smokers outperforming both non-smokers and daily smokers. The researchers purported to also investigate inhibitory control, but this was not consistent with their methods. Therefore, the gap here is identifying how nondaily smokers compare to non-smokers and daily smokers on inhibitory control and error processing as there was not a consistent methodological approach in previous research. One EEG study focusing on ERP components associated with inhibitory control and error processing will be presented in Chapter Four. 
TABLE 2.1: Overview of systematic review study demographics.

\begin{tabular}{|c|c|c|c|c|c|c|}
\hline Study & Participants & Mean age & $\%$ female & $\%$ white & Mean CPD & Mean FTCD \\
\hline \multirow[t]{2}{*}{ Billieux et al. (2010) } & 25 Light & Sample: 25.70 & Sample: $56 \%$ & $\mathrm{~N} / \mathrm{A}$ & $\mathrm{N} / \mathrm{A}$ & Sample: 2.07 \\
\hline & 25 Moderate & & & & & \\
\hline \multirow[t]{3}{*}{ Brinkmeyer et al. (2011) } & 907 Non-smokers & NS: 35.10 & NS: $60.40 \%$ & $\mathrm{~N} / \mathrm{A}$ & NS: N/A & NS: N/A \\
\hline & 463 Light & LS: 35.20 & LS: $56.50 \%$ & $\mathrm{~N} / \mathrm{A}$ & LS: 8.30 & LS: 1.11 \\
\hline & 353 Heavy & HS: 39.70 & HS: $52 \%$ & $\mathrm{~N} / \mathrm{A}$ & HS: 21.90 & LS: 5.64 \\
\hline \multirow[t]{3}{*}{ Brown et al. (2018) } & 25 Non-smokers & NS: 20.20 & NS: $76 \%$ & $\mathrm{~N} / \mathrm{A}$ & $\mathrm{N} / \mathrm{A}$ & NS: N/A \\
\hline & 25 Occasional & OS: 20.84 & OS: $84 \%$ & $\mathrm{~N} / \mathrm{A}$ & $\mathrm{N} / \mathrm{A}$ & OS: 0.73 \\
\hline & 20 Nicotine dependent & NDS: 23.15 & NDS: $44 \%$ & $\mathrm{~N} / \mathrm{A}$ & $\mathrm{N} / \mathrm{A}$ & NDS: 4.75 \\
\hline \multirow[t]{2}{*}{ Bühler et al. (2010) } & 21 Occasional & OS: 25.70 & $\mathrm{~N} / \mathrm{A}$ & $\mathrm{N} / \mathrm{A}$ & OS: 0.50 & OS: 0 \\
\hline & 21 Dependent & DS: 28.0 & $\mathrm{~N} / \mathrm{A}$ & $\mathrm{N} / \mathrm{A}$ & DS: 22.0 & DS: 6.50 \\
\hline \multirow[t]{3}{*}{ Carim-Todd et al. (2016) } & 25 Non-smokers & NS: 33.70 & NS: $56 \%$ & NS: $92 \%$ & NS: N/A & NS: N/A \\
\hline & 25 Non-dependent & NDS: 31.40 & NDS: $56 \%$ & NDS: 75\% & NDS: 2.61 & NDS: 0.63 \\
\hline & 23 Dependent & DS: 34.80 & DS: $61 \%$ & DS: $88 \%$ & DS: 17.21 & DS: 5.04 \\
\hline \multirow[t]{2}{*}{ Cochran et al. (2018) } & 33 Low nicotine dependence & LND: 26.52 & LND: $46 \%$ & LND: $48 \%$ & LND: 5.88 & Sample: 3.36 \\
\hline & 28 High nicotine dependence & HND: 30.86 & HND: $57 \%$ & HND: $43 \%$ & HND: 16.57 & \\
\hline
\end{tabular}


TABLE 2.1: Overview of systematic review study demographics.

\begin{tabular}{|c|c|c|c|c|c|c|}
\hline Study & Participants & Mean age & $\%$ female & $\%$ white & Mean CPD & Mean FTCD \\
\hline \multirow[t]{3}{*}{ Cui et al. (2012) } & 45 Light & LS: 41.60 & LS: $60 \%$ & LS: $49 \%$ & LS: 9.90 & LS: 3.00 \\
\hline & 61 Moderate & MS: 39.30 & MS: $39 \%$ & MS: $69 \%$ & MS: 19.30 & MS: 4.90 \\
\hline & 30 Heavy & HS: 47.00 & HS: $17 \%$ & HS: $80 \%$ & HS: 31.50 & HS: 6.30 \\
\hline \multirow[t]{3}{*}{ Dedandt et al. (2017) } & 28 Non-smokers & NS: 30.20 & NS: $86 \%$ & NS: N/A & NS: N/A & NS: N/A \\
\hline & 17 Low dependent & LDS: 28.80 & LDS: $47 \%$ & LDS: N/A & LDS: 12.00 & LDS: 2.80 \\
\hline & 15 Dependent & DS: 34.10 & DS: $93 \%$ & DS: N/A & DS: 10.80 & DS: 6.00 \\
\hline \multirow[t]{2}{*}{ Dickter et al. (2012) } & 27 Occasional & Sample: 19.80 & Sample: $71 \%$ & Sample: $71 \%$ & OS: $1.75 \mathrm{CPW}$ & OS: 0.27 \\
\hline & 34 Daily & & & & DS: $43.67 \mathrm{CPW}$ & DS: 2.07 \\
\hline \multirow[t]{2}{*}{ Field et al. (2006) } & 25 Infrequent & IS: 20.72 & IS: $40 \%$ & IS: N/A & IS: 4.18 & IS: $2.28 \mathrm{mFTQ}$ \\
\hline & 23 Frequent & FS: 23.26 & FS: $44 \%$ & FS: N/A & FS: 14.26 & FS: $3.39 \mathrm{mFTQ}$ \\
\hline \multirow[t]{4}{*}{ Fried et al. (2006) } & 64 Non-smokers & NS: N/A & NS: $45 \%$ & NS: N/A & NS: N/A & NS: N/A \\
\hline & 11 Former & FS: N/A & FS: $42 \%$ & FS: N/A & FS: N/A & FS: N/A \\
\hline & 19 Light & LS: N/A & LS: $47 \%$ & LS: N/A & LS: 4.90 & LS: N/A \\
\hline & 18 Heavy & HS: N/A & HS: $39 \%$ & HS: N/A & HS: 12.90 & HS: N/A \\
\hline Gantiva et al. (2019) & 35 Non-smokers & NS: 21.80 & NS: $49 \%$ & NS: N/A & NS: N/A & NS: N/A \\
\hline
\end{tabular}


TABLE 2.1: Overview of systematic review study demographics.

\begin{tabular}{|c|c|c|c|c|c|c|}
\hline Study & Participants & Mean age & $\%$ female & $\%$ white & Mean CPD & Mean FTCD \\
\hline & 35 Weekly & WS: 22.60 & WS: $32 \%$ & WS: N/A & WS: 0.42 & WS: N/A \\
\hline & 25 Daily & DS: 20.70 & DS: $49 \%$ & DS: N/A & DS: 7.80 & DS: N/A \\
\hline \multirow[t]{2}{*}{ Haight et al. (2012) } & 19 Occasional & OS: 19.20 & \multirow[t]{2}{*}{ Sample: $25.90 \%$} & OS: N/A & OS: $4.37 \mathrm{CPW}$ & OS: N/A \\
\hline & 34 Daily & DS: 20.10 & & DS: N/A & DS: $33.47 \mathrm{CPW}$ & DS: N/A \\
\hline \multirow[t]{2}{*}{ Harmsen et al. (2006) } & 47 Non-dependent & NDS: 43.50 & NDS: $36 \%$ & NDS: N/A & NDS: N/A & NDS: N/A \\
\hline & 61 Dependent & DS: 47.70 & DS: $36 \%$ & DS: N/A & DS: N/A & DS: N/A \\
\hline \multirow[t]{2}{*}{ Herman (1974) } & 54 Light & LS: 20.10 & \multirow[t]{2}{*}{ Sample: $0 \%$} & LS: N/A & LS: 9.10 & LS: N/A \\
\hline & 54 Heavy & HS: 20.20 & & HS: N/A & HS: 24.90 & HS: N/A \\
\hline \multirow[t]{3}{*}{ Heyman et al. (2006) } & 31 Non-smokers & NS: N/A & NS: N/A & NS: N/A & NS: N/A & NS: N/A \\
\hline & 21 Chippers & $\mathrm{C}: \mathrm{N} / \mathrm{A}$ & $\mathrm{C}: \mathrm{N} / \mathrm{A}$ & $C: N / A$ & C: $12 \mathrm{CPW}$ & C: 0.30 \\
\hline & 19 Regular & RS: N/A & RS: N/A & RS: N/A & RS: $97 \mathrm{CPW}$ & RS: 3.20 \\
\hline Hogarth et al. (2012a) & 22 Non-daily & NDS: 21.20 & NDS: $50 \%$ & NDS: N/A & NDS: 1.00 & NDS: N/A \\
\hline Study one & 22 Daily & DS: 21.30 & DS: 50\% & DS: N/A & DS: 8.00 & DS: N/A \\
\hline Hogarth et al. (2012a) & 18 Non-daily & NDS: 21.20 & NDS: $50 \%$ & NDS: N/A & NDS: 4.70 & NDS: N/A \\
\hline Study two & 18 Daily & DS: 21.50 & DS: $50 \%$ & DS: N/A & DS: 8.80 & DS: N/A \\
\hline
\end{tabular}


TABLE 2.1: Overview of systematic review study demographics.

\begin{tabular}{|c|c|c|c|c|c|c|}
\hline Study & Participants & Mean age & $\%$ female & $\%$ white & Mean CPD & Mean FTCD \\
\hline \multirow[t]{2}{*}{ Hogarth et al. (2012b) } & 32 Non-daily & NDS: 21.10 & NDS: $50 \%$ & NDS: N/A & NDS: 4.00 & NDS: N/A \\
\hline & 32 Daily & DS: 21.20 & DS: $50 \%$ & DS: N/A & DS: 9.20 & DS: N/A \\
\hline Hogarth et al. (2003) & 10 Non-smokers & NS: 20.50 & NS: $30 \%$ & NS: N/A & Sample: 16.80 & NS: N/A \\
\hline \multirow[t]{2}{*}{ Study one } & 6 Light & LS: 24.80 & LS: $67 \%$ & LS: N/A & & LS: N/A \\
\hline & 4 Heavy & HS: 21.50 & HS: 75\% & HS: N/A & & HS: N/A \\
\hline Hogarth et al. (2003) & 24 Non-smokers & NS: 22.20 & NS: $50 \%$ & NS: N/A & Sample: 13.80 & NS: N/A \\
\hline \multirow[t]{2}{*}{ Study two } & 25 Light & LS: 21.60 & LS: $48 \%$ & LS: N/A & & LS: N/A \\
\hline & 11 Heavy & HS: 22.20 & HS: $46 \%$ & HS: N/A & & HS: N/A \\
\hline \multirow[t]{3}{*}{ Johnson et al. (2007) } & 30 Non-smokers & NS: 33.00 & NS: $43 \%$ & NS: N/A & $\mathrm{NS}: \mathrm{N} / \mathrm{A}$ & NS: N/A \\
\hline & 30 Light & LS: 28.00 & LS: $43 \%$ & LS: N/A & LS: 5.90 & LS: 1.60 \\
\hline & 30 Heavy & HS: 30.90 & HS: $43 \%$ & HS: N/A & HS: 23.20 & HS: 6.60 \\
\hline \multirow[t]{2}{*}{ Knott et al. (2008) } & 21 Chippers & $C: 23.50$ & $C: 55 \%$ & $C: N / A$ & C: 3.60 & C: 2.00 \\
\hline & 11 Regular & RS: 24.00 & RS: $46 \%$ & RS: N/A & RS: 16.00 & RS: 3.75 \\
\hline \multirow[t]{2}{*}{ Lawn et al. (2015) } & 20 Occasional & OS: 22.90 & OS: $60 \%$ & OS: N/A & OS: 0.5 & OS: 0.00 \\
\hline & 20 Dependent & DS: 23.10 & DS: $50 \%$ & DS: N/A & DS: 16.70 & DS: 6.35 \\
\hline
\end{tabular}


TABLE 2.1: Overview of systematic review study demographics.

\begin{tabular}{|c|c|c|c|c|c|c|}
\hline Study & Participants & Mean age & $\%$ female & $\%$ white & Mean CPD & Mean FTCD \\
\hline \multirow[t]{2}{*}{ Lawn et al. (2018) } & 20 Occasional & OS: 22.60 & OS: $50 \%$ & OS: N/A & OS: 0.5 & OS: 0.00 \\
\hline & 20 Dependent & DS: 24.40 & DS: $50 \%$ & DS: N/A & DS: 16.50 & DS: 5.70 \\
\hline \multirow[t]{3}{*}{ Maynard et al. (2017) } & 19 Non-smokers & NS: 24.00 & NS: $47 \%$ & NS: N/A & NS: N/A & NS: N/A \\
\hline & 19 Weekly & WS: 21.30 & WS: 47\% & WS: N/A & WS: $10.90 \mathrm{CPW}$ & WS: 0.10 \\
\hline & 20 Daily & DS: 22.60 & DS: $45 \%$ & DS: N/A & DS: $81.50 \mathrm{CPW}$ & DS: 2.10 \\
\hline \multirow[t]{2}{*}{ Mogg et al. (2005) } & 19 Low dependence & LDS: 20.70 & LDS: $53 \%$ & LDS: N/A & LDS: 7.80 & LDS: $2.00 \mathrm{mFTQ}$ \\
\hline & 22 Moderate dependence & MDS: 24.20 & MDS: $50 \%$ & MDS: N/A & MDS: 13.40 & MDS: $4.10 \mathrm{mFTQ}$ \\
\hline \multirow[t]{2}{*}{ Motschman et al. (2016) } & 50 Occasional & OS: 20.20 & OS: $46 \%$ & OS: $74 \%$ & OS: 1.48 & OS: 0.12 \\
\hline & 55 Heavy & HS: 22.20 & HS: $46 \%$ & HS: 76\% & HS: 19.83 & HS: 5.52 \\
\hline \multirow[t]{2}{*}{ Nesic et al. (2011) } & 24 Low dependent & LDS: 24.00 & LDS: $50 \%$ & LDS: N/A & LDS: 6.80 & LDS: $3.40 \mathrm{FTQ}$ \\
\hline & 24 High dependent & HDS: 22.60 & HDS: $50 \%$ & HDS: N/A & HDS: 16.20 & HDS: $6.00 \mathrm{FTQ}$ \\
\hline Paelecke-Habermann & 25 Non-smokers & NS: 24.80 & NS: 72\% & NS: N/A & NS: N/A & NS: N/A \\
\hline & 20 Occasional & OS: 24.40 & OS: $85 \%$ & OS: N/A & OS: $34.60 \mathrm{CPM}$ & OS: 0.50 \\
\hline & 27 Dependent & DS: 25.90 & DS: $74 \%$ & DS: N/A & DS: $454.40 \mathrm{CPM}$ & DS: 2.93 \\
\hline Piasecki et al. (2017) & 44 Occasional & OS: 19.10 & OS: $48 \%$ & OS: $\mathrm{N} / \mathrm{A}$ & OS: 1.63 & OS: 0.27 \\
\hline
\end{tabular}


TABLE 2.1: Overview of systematic review study demographics.

\begin{tabular}{|c|c|c|c|c|c|c|}
\hline Study & Participants & Mean age & $\%$ female & $\%$ white & Mean CPD & Mean FTCD \\
\hline & 46 Daily & DS: 19.80 & DS: $46 \%$ & DS: N/A & DS: 8.93 & DS: 2.07 \\
\hline \multirow[t]{3}{*}{ Poltavski et al. (2013) } & 92 Non-smokers & Sample: 20.00 & Sample: $83 \%$ & Sample: $88 \%$ & NS: N/A & NS: N/A \\
\hline & 73 Light & & & & LS: N/A & LS: 1.35 \\
\hline & 17 Heavy & & & & HS: N/A & HS: 3.90 \\
\hline \multirow[t]{3}{*}{ Rass et al. (2016) } & 30 Non-smokers & NS: 25.20 & NS: $53 \%$ & NS: $67 \%$ & NS: N/A & NS: N/A \\
\hline & 31 Non-daily & NDS: 23.90 & NDS: $61 \%$ & NDS: 77\% & NDS: 3.60 & NDS: 0.50 \\
\hline & 22 Daily & DS: 27.00 & DS: $41 \%$ & DS: $82 \%$ & DS: 16.10 & DS: 5.40 \\
\hline \multirow[t]{3}{*}{ Rass et al. (2014) } & 30 Non-smokers & NS: 25.20 & NS: $53 \%$ & NS: $67 \%$ & NS: N/A & NS: N/A \\
\hline & 31 Non-daily & NDS: 23.90 & NDS: $61 \%$ & NDS: 77\% & NDS: 3.60 & NDS: 0.50 \\
\hline & 22 Daily & DS: 27.00 & DS: $41 \%$ & DS: $82 \%$ & DS: 16.10 & DS: 5.40 \\
\hline \multirow[t]{3}{*}{ Rezvanfard et al. (2010) } & 30 Non-smokers & NS: 24.80 & NS: $0 \%$ & NS: N/A & NS: N/A & NS: N/A \\
\hline & 37 Lightly dependent & LDS: 24.20 & LDS: $0 \%$ & LDS: N/A & LDS: 7.27 & LDS: $4.37 \mathrm{mFTQ}$ \\
\hline & 22 Heavily dependent & HDS: 24.00 & HDS: $0 \%$ & HDS: N/A & HDS: 15.68 & HDS: $9.12 \mathrm{mFTQ}$ \\
\hline \multirow[t]{2}{*}{ Sayette et al. (2001) } & 60 Tobacco chippers & TC: 24.10 & Sample: $53 \%$ & Sample: $77 \%$ & TC: 3.75 & TC: N/A \\
\hline & 67 Heavy & HS: 25.30 & & & HS: 24.85 & HS: N/A \\
\hline
\end{tabular}


TABLE 2.1: Overview of systematic review study demographics.

\begin{tabular}{|c|c|c|c|c|c|c|}
\hline Study & Participants & Mean age & $\%$ female & $\%$ white & Mean CPD & Mean FTCD \\
\hline \multirow[t]{3}{*}{ Stevens et al. (1976) } & 67 Non-smokers & Sample: 20.30 & Sample: $0 \%$ & NS: N/A & NS: N/A & NS: N/A \\
\hline & 23 Light & & & LS: N/A & LS: 6.65 & LS: N/A \\
\hline & 27 Heavy & & & HS: N/A & HS: 18.48 & HS: N/A \\
\hline \multirow[t]{3}{*}{ Stillwell et al. (2012) } & 6777 Non-smokers & Sample: 23.40 & Sample: $57 \%$ & NS: N/A & NS: N/A & NS: N/A \\
\hline & 669 Non-daily & & & LDS: N/A & LDS: N/A & LDS: 7.06 CDS-5 \\
\hline & 1592 Daily & & & DS: N/A & DS: N/A & DS: 16.03 CDS-5 \\
\hline \multirow[t]{3}{*}{ Sweitzer et al. (2008) } & 145 Never & Sample: 45.30 & NS: N/A & Sample: $87 \%$ & NS: N/A & NS: N/A \\
\hline & 50 Low dependence & & LDS: $50 \%$ & & LDS: 10.56 & LDS: 1.00 \\
\hline & 47 High dependence & & HDS: $49 \%$ & & HDS: 18.83 & HDS: 4.11 \\
\hline \multirow[t]{3}{*}{ Tait et al. (2000) } & 24 Non-smokers & NS: 22.00 & Sample: $71.40 \%$ & NS: N/A & NS: N/A & NS: N/A \\
\hline & 22 Light & LS: 22.00 & & LS: N/A & LS: 6.50 & LS: N/A \\
\hline & 19 Heavy & HS: 25.00 & & HS: N/A & HS: 23.00 & HS: N/A \\
\hline Venugopalan & 15 Low frequency early & LFE: 19.90 & LFE: $60 \%$ & LFE: N/A & LFE: 2.70 & LFE: 2.50 \\
\hline \multirow[t]{2}{*}{ et al. (2011) } & 16 Low frequency stable & LFS: 21.80 & LFS: $63 \%$ & LFS: N/A & LFS: 4.90 & LFS: 3.10 \\
\hline & 16 High frequency stable & HFS: 21.00 & HFS: $38 \%$ & HFS: N/A & HFS: 12.60 & HFS: 5.30 \\
\hline
\end{tabular}


TABLE 2.1: Overview of systematic review study demographics.

\begin{tabular}{|c|c|c|c|c|c|c|}
\hline Vollstädt-Klein & 41 Non-smokers & NS: 32.00 & NS: 63\% & NS: N/A & NS: N/A & NS: N/A \\
\hline et al. (2011) & 15 Heavy & HS: 37.10 & & HS: N/A & HS: 24.13 & HS: 6.07 \\
\hline \multirow[t]{2}{*}{ Wardell et al. (2018) } & 91 Non-smokers & NS: 19.70 & NS: $58 \%$ & NS: $50 \%$ & NS: N/A & NS: N/A \\
\hline & 14 Daily & DS: 19.70 & DS: $71 \%$ & DS: $64 \%$ & DS: 4.67 & DS: N/A \\
\hline \multirow[t]{3}{*}{ Yakir et al. (2007) } & 151 Non-smokers & NS: 23.10 & Sample: $100 \%$ & NS: $47 \%$ & NS: N/A & NS: N/A \\
\hline & 40 Past & PS: 24.70 & & PS: 39\% & PS: N/A & PS: N/A \\
\hline & 46 Occasional & OS: 23.40 & & OS: $78 \%$ & OS: N/A & OS: $1.00 \mathrm{FTQ}$ \\
\hline
\end{tabular}


TABLE 2.2: Overview of systematic review studies smoking definitions and labels.

\begin{tabular}{|c|c|c|c|c|}
\hline Study & Lighter smoking criteria & Heavier smoking criteria & Non-smoking control? & Only daily smokers? \\
\hline \multirow[t]{2}{*}{ Billieux et al. (2010) } & Light & Moderate & \multirow[t]{2}{*}{ No } & \multirow[t]{2}{*}{ Not specified } \\
\hline & $<10 \mathrm{CPD}$ & 10-20 CPD & & \\
\hline \multirow[t]{2}{*}{ Brinkmeyer et al. (2011) } & Light & Heavy & \multirow[t]{2}{*}{ Yes } & \multirow[t]{2}{*}{ No } \\
\hline & $<4$ FTCD & $\geq 4$ FTCD & & \\
\hline \multirow[t]{2}{*}{ Brown et al. (2018) } & Occasional & Nicotine dependent & \multirow[t]{2}{*}{ Yes } & \multirow[t]{2}{*}{ No } \\
\hline & $<3$ FTCD & $\geq 3$ FTCD & & \\
\hline \multirow[t]{3}{*}{ Bühler et al. (2010) } & Occasional & Dependent & \multirow[t]{3}{*}{ No } & \multirow[t]{3}{*}{ No } \\
\hline & 0 FTCD & DSM-IV nicotine dependence & & \\
\hline & $<6 \mathrm{CPW}$ & $\geq 5$ FTCD & & \\
\hline \multirow[t]{4}{*}{ Carim-Todd et al. (2016) } & Non-dependent & Dependent & \multirow[t]{4}{*}{ Yes } & \multirow[t]{4}{*}{ No } \\
\hline & 1-5 CPD & $\geq 15 \mathrm{CPD}$ & & \\
\hline & $\geq 2$ days per week & $\mathrm{CO}>10 \mathrm{ppm}$ & & \\
\hline & $\leq 4 \mathrm{FTCD}$ & $\geq 5$ FTCD & & \\
\hline \multirow[t]{2}{*}{ Cochran et al. (2018) } & Low nicotine dependence & High nicotine dependence & \multirow[t]{2}{*}{ No } & \multirow[t]{2}{*}{ No } \\
\hline & $\leq 3 \mathrm{FTCD}$ & $>3$ FTCD & & \\
\hline
\end{tabular}


TABLE 2.2: Overview of systematic review studies smoking definitions and labels.

\begin{tabular}{|c|c|c|c|c|}
\hline Study & Lighter smoking criteria & Heavier smoking criteria & Non-smoking control? & Only daily smokers? \\
\hline \multirow[t]{4}{*}{ Cui et al. (2012) } & Light & Moderate & No & Yes \\
\hline & 5-14 CPD & $15-24 \mathrm{CPD}$ & & \\
\hline & & Heavy & & \\
\hline & & $\geq 25 \mathrm{CPD}$ & & \\
\hline \multirow[t]{2}{*}{ Dedandt et al. (2017) } & Low dependent & Dependent & Yes & Not specified \\
\hline & 1-4 FTCD & $\geq 5$ FTCD & & \\
\hline \multirow[t]{2}{*}{ Dickter et al. (2012) } & Occasional & Daily & No & No \\
\hline & Non-daily smoking & $\geq 1 \mathrm{CPD}$ & & \\
\hline \multirow[t]{2}{*}{ Field et al. (2006) } & Infrequent & Frequent & No & No \\
\hline & $<10 \mathrm{CPD}$ & $\geq 10 \mathrm{CPD}$ & & \\
\hline \multirow[t]{2}{*}{ Fried et al. (2006) } & Light & Heavy & Yes & Yes \\
\hline & $<9$ CPD & $>9 \mathrm{CPD}$ & & \\
\hline \multirow[t]{3}{*}{ Gantiva et al. (2019) } & Weekly & Daily & Yes & No \\
\hline & $\geq 1 \mathrm{CPW}$ & $\geq 1 \mathrm{CPD}$ & & \\
\hline & Not daily & & & \\
\hline
\end{tabular}


TABLE 2.2: Overview of systematic review studies smoking definitions and labels.

\begin{tabular}{|c|c|c|c|c|}
\hline Study & Lighter smoking criteria & Heavier smoking criteria & Non-smoking control? & Only daily smokers? \\
\hline \multirow[t]{2}{*}{ Haight et al. (2012) } & Occasional & Daily & No & No \\
\hline & Non-daily smoking & $\geq 1 \mathrm{CPD}$ & & \\
\hline \multirow[t]{2}{*}{ Harmsen et al. (2006) } & Non-dependent & Dependent & No & Yes \\
\hline & Non-dependent (M-CIDI) & Dependent (M-CIDI) & & \\
\hline \multirow[t]{2}{*}{ Herman (1974) } & Light & Heavy & No & Yes \\
\hline & $\leq 15 \mathrm{CPD}$ & $\geq 20 \mathrm{CPD}$ & & \\
\hline \multirow[t]{2}{*}{ Heyman et al. (2006) } & Chippers & Regular & Yes & No \\
\hline & 2-39 CPW & $\geq 40 \mathrm{CPW}$ & & \\
\hline \multirow[t]{2}{*}{ Hogarth et al. (2012a) } & Non-daily & Daily & No & No \\
\hline & Non-daily smoking & Daily smoking & & \\
\hline \multirow[t]{2}{*}{ Hogarth et al. (2012b) } & Non-daily & Daily & No & No \\
\hline & Non-daily smoking & Daily smoking & & \\
\hline \multirow[t]{2}{*}{ Hogarth et al. (2003) } & Light & Heavy & Yes & Yes \\
\hline & $<20 \mathrm{CPD}$ & $\geq 20 \mathrm{CPD}$ & & \\
\hline Johnson et al. (2007) & Light & Heavy & Yes & Yes \\
\hline
\end{tabular}


TABLE 2.2: Overview of systematic review studies smoking definitions and labels.

\begin{tabular}{|c|c|c|c|c|}
\hline Study & Lighter smoking criteria & Heavier smoking criteria & Non-smoking control? & Only daily smokers? \\
\hline & 1-10 CPD & $\geq 20 \mathrm{CPD}$ & & \\
\hline \multirow[t]{3}{*}{ Knott et al. (2008) } & Chippers & Regular & No & No \\
\hline & $\leq 5 \mathrm{CPD}$ & $\geq 15 \mathrm{CPD}$ & & \\
\hline & $\geq 2$ smoking days per week & & & \\
\hline \multirow[t]{6}{*}{ Lawn et al. (2015) } & Occasional & Dependent & No & No \\
\hline & $\leq 5 \mathrm{CPW}$ & $\geq 10 \mathrm{CPD}$ & & \\
\hline & $\geq 1 \mathrm{CPM}$ & $\geq 5$ FTCD & & \\
\hline & $\mathrm{FTCD}=0$ & DSM-V tobacco use disorder & & \\
\hline & No DSM-5 tobacco use disorder & & & \\
\hline & Never smoked daily & & & \\
\hline \multirow[t]{3}{*}{ Lawn et al. (2018) } & Occasional & Dependent & No & No \\
\hline & 0.5-5 CPW & $\geq 10 \mathrm{CPD}$ & & \\
\hline & 0 FTCD & $\geq 5$ FTCD & & \\
\hline \multirow[t]{2}{*}{ Maynard et al. (2017) } & Weekly & Daily & Yes & No \\
\hline & $\geq 1 \mathrm{CPW}$ & $\geq 5 \mathrm{CPD}$ & & \\
\hline
\end{tabular}


TABLE 2.2: Overview of systematic review studies smoking definitions and labels.

\begin{tabular}{|c|c|c|c|c|}
\hline Study & Lighter smoking criteria & Heavier smoking criteria & Non-smoking control? & Only daily smokers? \\
\hline & Non-daily smoking & Smoke within hour of waking & & \\
\hline \multirow[t]{2}{*}{ Mogg et al. (2005) } & Low dependence & Moderate dependence & No & Yes \\
\hline & $<3$ FTCD & $\geq 3$ FTCD & & \\
\hline \multirow[t]{5}{*}{ Motschman et al. (2016) } & Occasional & Heavy & No & No \\
\hline & $\leq 5$ smoking days in last 30 & Daily smoking in last 30 & & \\
\hline & $\leq 5 \mathrm{CPD}$ & $\geq 15 \mathrm{CPD}$ & & \\
\hline & Never smoked $>14$ days per month & $\geq 10$ ppm CO & & \\
\hline & $<10$ ppm CO & & & \\
\hline \multirow[t]{2}{*}{ Nesic et al. (2011) } & Low dependent & High dependent & No & Yes \\
\hline & $\leq 4 \mathrm{FTQ}$ & $\geq 5$ FTQ & & \\
\hline \multirow{4}{*}{$\begin{array}{l}\text { Paelecke-Habermann } \\
\text { et al. (2013) }\end{array}$} & Occasional & Dependent & Yes & No \\
\hline & Non-daily smoking & DSM-IV dependence & & \\
\hline & $\leq 3$ withdrawal symptoms & Daily smoking & & \\
\hline & & $\geq 4$ withdrawal symptoms & & \\
\hline Piasecki et al. (2017) & Occasional & Daily & No & No \\
\hline
\end{tabular}


TABLE 2.2: Overview of systematic review studies smoking definitions and labels.

\begin{tabular}{|c|c|c|c|c|}
\hline Study & Lighter smoking criteria & Heavier smoking criteria & Non-smoking control? & Only daily smokers? \\
\hline & $\geq 1$ smoking day past month & $\geq 5 \mathrm{CPD}$ & & \\
\hline & Non-daily smoking & & & \\
\hline \multirow[t]{2}{*}{ Poltavski et al. (2013) } & Light & Heavy & Yes & No \\
\hline & $<10 \mathrm{CPD}$ & $\geq 10 \mathrm{CPD}$ & & \\
\hline \multirow[t]{5}{*}{ Rass et al. (2016) } & Non-daily & Daily & Yes & No \\
\hline & Smoked $\geq 3$ years & $\geq 12$ months daily smoking & & \\
\hline & $<27$ smoking days per month & $\geq 4 \mathrm{FTCD}$ & & \\
\hline & $\geq 10$ smoking days in past 90 & & & \\
\hline & or $\geq 20$ cigarettes in past 90 & & & \\
\hline \multirow[t]{6}{*}{ Rass et al. (2014) } & Non-daily & Daily & Yes & No \\
\hline & Smoked $\geq 3$ years & $\geq 12$ months daily smoking & & \\
\hline & $<27$ smoking days per month & $\geq 4$ FTCD & & \\
\hline & $\geq 10$ smoking days in past 90 & $<25 \mathrm{CPD}$ & & \\
\hline & or $\geq 20$ cigarettes in past 90 & & & \\
\hline & $<4$ FTCD & & & \\
\hline
\end{tabular}


TABLE 2.2: Overview of systematic review studies smoking definitions and labels.

\begin{tabular}{|c|c|c|c|c|}
\hline Rezvanfard et al. (2010) & Lightly dependent & Heavily dependent & Yes & Yes \\
\hline \multirow[t]{2}{*}{ Sayette et al. (2001) } & Tobacco chippers & Heavy & No & No \\
\hline & $\geq 2$ smoking days per week & $\geq 21 \mathrm{CPD}$ & & \\
\hline \multirow[t]{2}{*}{ Stevens et al. (1976) } & Light & Heavy & Yes & Yes \\
\hline & $<13 \mathrm{CPD}$ & $\geq 13 \mathrm{CPD}$ & & \\
\hline \multirow[t]{2}{*}{ Stillwell et al. (2012) } & Light & Daily & Yes & No \\
\hline & Non-daily smoking & Daily smoking & & \\
\hline Tait et al. (2000) & $\leq 10 \mathrm{CPD}$ & $\geq 20 \mathrm{CPD}$ & & \\
\hline Venugopalan & Low frequency early & High frequency stable & No & No \\
\hline et al. (2011) & $\leq 5 \mathrm{CPD}<1$ year & $\geq 10 \mathrm{CPD}$ & & \\
\hline
\end{tabular}


TABLE 2.2: Overview of systematic review studies smoking definitions and labels.

\begin{tabular}{|c|c|c|c|c|}
\hline Study & Lighter smoking criteria & Heavier smoking criteria & Non-smoking control? & Only daily smokers? \\
\hline & Non-daily smoking & & & \\
\hline & Low frequency stable & & & \\
\hline & $\leq 5 \mathrm{CPD} \geq 3$ years & & & \\
\hline & Non-daily smoking & & & \\
\hline Vollstädt-Klein & Light & Heavy & Yes & Yes \\
\hline et al. (2011) & $<20 \mathrm{CPD}$ & $\geq 20 \mathrm{CPD}$ & & \\
\hline \multirow[t]{3}{*}{ Wardell et al. (2018) } & Non-daily & Daily & Yes & No \\
\hline & Non-daily smoking & Daily smoking & & \\
\hline & $\geq 1$ cigarette previous 90 days & & & \\
\hline \multirow[t]{2}{*}{ Yakir et al. (2007) } & Occasional & Current & Yes & No \\
\hline & Smoking from time to time & Regular smoking & & \\
\hline
\end{tabular}

Abbreviations. CO (Carbon Monoxide); CPD (cigarettes per day); CPM (cigarettes per month); CPW (cigarettes per week); FTCD (Fagerström Test for Cigarette Dependence); FTQ (Fagerström Tolerance Questionnaire); M-CIDI (Munich Composite International Diagnostic Interview); mFTQ (modified Fagerström Tolerance Questionnaire). 


\section{Chapter 3}

\section{Attentional Bias Towards Smoking}

\section{Cues}

\subsection{Chapter overview}

The systematic review in Chapter Two found that there was mixed research on attentional bias in different smoking groups. Some studies found that lighter smokers showed greater attentional bias to smoking cues, whereas other studies found that heavier smokers showed greater attentional bias. The iRISA model of addiction outlines over-active drive processes as one component of problematic drug use, with cues associated with drug use having increased salience. Therefore, if attentional bias towards drug cues has a role in the maintenance of drug use, then it is important to investigate whether attentional bias is greater in daily or non-daily smokers. Chapter Three presents two studies investigating attentional bias in daily and non-daily smokers.

As a reminder from Chapter One, the smoking groups in the first study presented in this chapter (Study One) are referred to as light and heavy smokers instead of daily and non-daily smokers. This was due to the design of Study One being modelled on previous attentional bias research that used the FTCD score to dichotomise smokers and the study was planned before the systematic review was complete. Data on the number of smoking days was not collected, meaning it was not possible to reclassify them as daily and non-daily smokers. However, for the remaining studies in this thesis, the smoking groups are consistently referred to as daily and non-daily smokers. 


\subsection{Introduction}

Attentional bias is the tendency to fixate attention on environmental cues associated with smoking (Field and Cox 2008). It has been shown to have a positive but small relationship with the experience of craving (Field, Munafó and Franken 2009) and it is predictive of abstinence after one week of cessation (Powell et al. 2010). Previous research has consistently shown that in comparison to non-smokers, smokers exhibit greater attentional bias towards smoking-related cues (Ehrman, Robbins and Bromwell 2002; Mogg and Bradley 2002; Mogg, Bradley and Field 2003; Kang, Chang and Jahng 2012; Baschnagel 2013). However, these studies do not investigate the heterogeneity within different types of smokers. When studies have taken this heterogeneity into account, they have produced conflicting results. For example, some studies have shown that lighter smokers exhibit greater attentional bias towards smoking cues than heavier smokers (Bradley, Mogg and Wright 2003; Hogarth et al. 2003; Mogg et al. 2005). On the other hand, other studies have shown that heavier smokers exhibit greater attentional bias than lighter smokers (Zack, Belsito and Scher 2001; Chanon, Sours and Boettiger 2010; Vollstädt-Klein et al. 2011). These studies vary in how they define their smoking groups, and employ a range of different methods. The most common measure of attentional bias was the visual probe task. Crucially, despite using the same task, there were inconsistent design features that make it difficult to conclude whether the conflicting results were due to the differences in the samples used, or whether they were due to inconsistencies in the methods. For the first study in this thesis, the differences in designing the visual probe task were investigated by focusing on three key features: the stimulus onset asynchrony (SOA), differentiating vigilance from disengagement, and the use of individualised smoking stimuli.

The visual probe task infers attention through differences in response time (RT). Two images are presented and when they disappear, the participant is required to indicate the location of a small probe that replaced the location of one of the images. Faster RTs to particular types of stimuli are thought to reflect selective attention to a particular location. If the participant is already looking in the location that the probe appears in, the idea is that they can see it sooner and provide a faster response (Field and Cox 2008). As the location of attention is inferred through differences in RT after 
the stimuli disappear, the presentation time of the stimuli can be manipulated to allow the participant more or less time to attend to the screen. Short SOAs of 200ms or less are thought to measure involuntary attentional processes as there is only enough time for one fixation before the images disappear and a response is required (Field and Cox 2008). On the other hand, longer SOAs of 500ms or more target voluntary attention as multiple fixations can be made between stimuli. Previous research focusing on light and heavy smokers used a single SOA, with one study displaying the images for 500ms (Vollstädt-Klein et al. 2011), and two displaying the images for 2000ms (Hogarth et al. 2003; Mogg et al. 2005). This creates an element of uncertainty as both studies using a $2000 \mathrm{~ms}$ SOA found that light smokers exhibited attentional bias towards smoking cues, and the study using a 500ms SOA found that heavy smokers exhibited attentional bias. The conflict in results may be due to attentional bias being expressed through different processes of attention in light and heavy smokers. In addition, none of the studies used a very short SOA to measure involuntary attentional processes. Chanon et al. (2010) found that attentional bias was greater in smokers than non-smokers when a $200 \mathrm{~ms}$ SOA was used in comparison to a 550ms SOA. Therefore, to investigate whether the conflict in results between light and heavy smokers is due to attentional bias being expressed in different processes of attention, it is important to use multiple SOAs in the visual probe task. Inspired by the design of Field, Mogg and Zetteler (2004), the current study included three SOAs of 200ms, 500ms, and 2000ms to explore if attentional bias is expressed in different attentional processes for light and heavy smokers.

The second consideration is distinguishing whether attentional bias is the product of vigilance or a difficulty with disengagement. The assumption behind the visual probe task is that faster RTs to smoking stimuli indicate that people's attention has been oriented towards the smoking stimuli. However, the same difference in RT could also be produced by slower responses to neutral stimuli in the presence of smoking stimuli (Koster, Crombez and Verschuere 2004). Vigilance is defined as orienting attention towards smoking cues when they are presented with a neutral cue (Salemink, van den Hout, and Kindt 2010). Conversely, a difficulty to disengage with smoking cues is defined as delayed responding to probes replacing neutral cues due to attention being preoccupied with the smoking cues presented at the same time. This is a subtle 
but important distinction as the conceptualisation of attentional bias being expressed as a reflexive or involuntary response would be consistent with vigilance. A difficulty to disengage with smoking cues would be consistent with a voluntary allocation of attention. It does not appear that this consideration has been taken into account in previous research in smokers. The current study included three different trial conditions to distinguish between vigilance and a difficult to disengage. The first is when the probe replaces a smoking-image next to a neutral image (smoking-neutral or SN trials). The second is when the probe replaces a neutral image next to a smoking image (neutral-smoking or NS trials). These two trial types form the standard version of the visual probe task used in previous research to calculate a total bias index. The third condition is when two neutral images are presented together (neutral-neutral or NN trials). This means if RTs are faster to smoking-neutral trials than to neutral-neutral trials, then attentional bias can be inferred to be the product of vigilance. On the other hand, if neutral-smoking trials produce slower RTs than neutral-neutral trials, then attentional bias can be inferred to be the product of a difficulty to disengage attention (Salemink et al. 2010).

The final design consideration is the internal consistency of the visual probe task. Despite being commonly used in previous research, its reliability has been questioned (Schmukle 2005; Ataya, Adams and Mullings 2012). Previous research has reported estimates between 0 (Waechter, Nelson, and Wright 2014) and .28 (Schmukle 2005). This is problematic as low internal consistency not only reduces its validity as a measure, but it also decreases statistical power (Henson 2001). Therefore, it is important to take into consideration how the visual probe task can be used to reliably measure attentional bias. In a group of alcohol drinkers, the internal consistency of the visual probe task improved with the inclusion of individualised stimuli (Christiansen, Mansfield, and Duckworth 2015). Cronbach's alpha improved from .19 to .73 when the task contained images of the participant's drink of choice. Most studies use a set of standardised images for all of their participants. However, it is not realistic to expect all participants to have the same preferences. Salience is not a universal property, but rather it is specific to the individual and their experience of using their drug of choice (Hester and Luijten 2014). Individualised cues are predicted to increase cue reactivity for smokers, but little research has been done on the topic (Jasinska, Stein and Kaiser 
2014). The participants in Study One completed a version of the visual probe task containing either images of roll-up cigarettes or of manufactured cigarettes depending on their self-reported preferences.

Study One aimed to clarify the presence of attentional bias towards smokingrelated cues in light and heavy smokers by focusing on three key design features of the visual probe task. The hypotheses and sampling plan were pre-registered on the Open Science Framework (OSF; https://osf.io/h9ex7/). First, light smokers were expected to exhibit greater attentional bias towards smoking stimuli, but heavy smokers and non-smokers were not. Second, light smokers were predicted to exhibit attentional bias towards smoking stimuli specifically through vigilance and not a difficulty to disengage. No a priori predictions were made for how attentional bias would differ across the different SOA conditions.

\subsection{Study One}

\subsubsection{Method}

\subsubsection{Design}

A $3 \times 3 \times 3$ mixed quasi-experimental design was used as the smoking groups were self-selected. This consisted of one between-subjects Independent Variable (IV) of smoking group with three levels: light smoker, heavy smoker, or non-smoker. Light smokers were defined as those with FTCD scores of two or fewer, and heavy smokers had scores of three or more. In addition, there were two within-subject IVs. The SOA of the visual probe task with three levels $(200 \mathrm{~ms}, 500 \mathrm{~ms}, 2000 \mathrm{~ms})$ and the trial type with three levels (neutral-neutral, smoking-neutral, neutral-smoking). The Dependent Variable (DV) was the mean RT (ms) to respond to the probes replacing one of the images.

\subsubsection{Participants}

Based on the sample sizes of previous research in attentional bias (e.g. Field et al. 2004), the sampling plan for Study One was to recruit 20 non-smokers and 40 smokers. Consistent with this plan, a total of 20 non-smokers, 22 light smokers, and 18 
heavy smokers were recruited from university staff and students. Inclusion criteria included all participants should not possess any neurological deficits that may impact their ability to complete a computer-based task and have normal or corrected-normal vision. An additional non-smoker was originally recruited but they were excluded for reporting to have smoked 50 cigarettes in their lifetime. To remain consistent with the sampling plan, an additional participant was recruited to ensure 20 non-smokers were included. Participants signed up to the study through an undergraduate research participation scheme or responded to adverts placed around the university campus. Participants received research participation credits or a $£ 5$ store voucher in exchange for their time.

\subsubsection{Materials}

Fagerström Test for Cigarette Dependence (FTCD)

The FTCD (Heatherton et al. 1991; Fagerström 2011) is a self-report questionnaire of nicotine dependence. Six questions assess behaviours associated with dependence such as "How soon after you wake up do you smoke your first cigarette?" and "Which cigarette would you hate most to give up?". Participants complete the questionnaire using a fixed rating scale with answers corresponding to points. A greater number of points indicates a greater level of nicotine dependence, with scores ranging from zero to ten. The FTCD has good test-retest reliability and criterion validity (Pomerleau, Carton and Lutzke 1994), but despite being commonly used, internal consistency may be questionable. Previous studies have estimated Cronbach's alpha internal consistency to range between .61 (Heatherton et al. 1991) and .67 (Haddock et al. 1999). The internal consistency of the FTCD in Study One was lower than that reported in previous studies $(\alpha=.41,95 \% \mathrm{CI}=[.06, .61])$.

\section{Positive and Negative Affect Schedule (PANAS)}

The PANAS is a questionnaire to assess mood using two 10 item sub-scales measuring positive and negative affect (Watson, Clark, and Tellegen 1988). A total score is calculated separately for each scale, with a range of 10-50 for each. A lower score indicates low positive or negative affect, and a higher score indicates high positive or negative affect. Participants rate how they feel by rating different adjectives on a scale 
of one (very slightly or not at all) to five (extremely). Words relating to positive affect include interested, alert, enthusiastic, and determined. Words relating to negative affect include distressed, hostile, afraid, and jittery. Previous studies have reported high levels of internal consistency for both the positive $(\alpha=.89)$ and negative affect $(\alpha=$ .85) sub-scales (Crawford and Henry 2004). Internal consistency in Study One before completing the visual probe task was similar to previous estimates for positive affect $(\alpha=.84,95 \% \mathrm{CI}=[.76, .89)$, but negative affect $(\alpha=.79,95 \% \mathrm{CI}=[.59, .88])$ was slightly lower.

The Brief Questionnaire of Smoking Urges (QSU-brief)

The QSU-brief assesses the craving associated with the use of cigarettes (Cox, Tiffany, and Christen 2001). The questionnaire includes 10 statements which are answered using a seven-point Likert scale ranging from one (strongly disagree) to seven (strongly agree). It can be broken down into two factors that both contain five statements. Factor one measures the rewarding properties of smoking, and factor two measures the perceived relief from negative affect after smoking. The statements from each factor are summed to produce a score ranging from 5-35 for each factor. A high score indicates high craving. An example for a statement in factor one is "I have a desire for a cigarette right now". An example for a statement in factor two is "I could control things better right now if I could smoke". In a non-clinical sample, factor one $(\alpha=.96)$ and factor two $(\alpha=.93)$ both had very high levels of internal consistency (Cox et al. 2001). The estimate from this study when the scale was completed before the visual probe task is consistent with those reported by Cox et al. (2001) for factor one ( $\alpha=.91$, $95 \% \mathrm{CI}=[.86, .95])$, but marginally lower for factor two $(\alpha=.87,95 \% \mathrm{CI}=[.67, .94])$, despite still showing good internal consistency.

\section{Wisconsin Inventory of Smoking Dependence Motives (WISDM)}

The WISDM explores 13 different motives associated with smoking cigarettes that are assessed by 68 statements (Piper et al. 2004). There are 13 sub-scales relating to different smoking motives and each statement is rated with a seven-point Likert scale ranging from one (not true of me at all) to seven (extremely true of me). Each subscale receives a score by calculating the mean response in that sub-scale and can range 
from one to seven. A total score for the WISDM-68 can range between 13 and 91 which can be calculated by summing the means of each sub-scale, with a higher score indicating greater smoking dependence. Each sub-scale represents a primary or secondary smoking motive (Shiffman et al. 2012a).

Primary motives can be compared to the FTCD through targeting physical indicators of dependence such as tolerance, craving, automaticity, and loss of control over smoking opportunities (such as home or work smoking restrictions). An example statement for the tolerance motive is "I usually want to smoke right after I wake up". On the other hand, secondary motives target motivational aspects of dependence such as cue exposure, social goads, and positive reinforcement. Rather than explaining smoking behaviour purely through the avoidance of experiencing nicotine withdrawal, these motives target the supplementary aspects of smoking that drive people's behaviour. These include smoking while socialising, helping to control weight, and the pleasurable sensory sensations of smoking. An example statement for the cue exposure motive is "There are particular sights and smells that trigger strong urges to smoke". Previous estimates for the internal consistency for each sub-scale range from good to excellent with Cronbach's alpha scores ranging between .73 to .95 (Piper et al. 2004). The Cronbach's Alpha values for each sub-scale of the WISDM in this study are presented in Table 3.1. Values ranged from .67 to .94 with most values being highly consistent and within the same range as that reported by Piper et al. (2004). However, the cue exposure sub-scale $(\alpha=.67)$ displayed the lowest internal consistency in comparison to the other sub-scales reported in this study and Piper et al. (2004).

\subsubsection{Measures}

\section{Carbon monoxide}

To assess the amount of carbon monoxide (CO) present in the participant's lungs, a MicroCO Monitor (CareFusion Corporation, USA) was used. This handheld device measures the exhaled concentration of $\mathrm{CO}$ in the lungs in parts per million (ppm). Each participant was required to hold their breath for 20 seconds before blowing into a disposable mouthpiece. Measuring exhaled $\mathrm{CO}$ in one session has been shown to be an effective measure of cigarette use in both light and heavy smokers (Wray, Gass, and Miller 2015). 
TABLE 3.1: Internal consistency estimates using Cronbach's alpha for each sub-scale of the WISDM-68.

\begin{tabular}{cccc}
\hline Sub-scale & Lower CI & Alpha & Upper CI \\
\hline Affiliative Attachment & .78 & .90 & .95 \\
\hline Automaticity & .83 & .90 & .94 \\
\hline Loss of Control & .66 & .81 & .89 \\
\hline Behavioural Choice & .59 & .77 & .86 \\
\hline Cognitive Enhancement & .80 & .89 & .93 \\
\hline Craving & .74 & .83 & .89 \\
\hline Cue Exposure & .47 & .67 & .78 \\
\hline Negative Reinforcement & .74 & .83 & .89 \\
\hline Positive Reinforcement & .78 & .87 & .92 \\
\hline Social Goads & .70 & .86 & .94 \\
\hline Sensory Processes & .84 & .90 & .94 \\
\hline Tolerance & .49 & .71 & .84 \\
\hline Weight Control & .89 & .94 & .97
\end{tabular}

\section{International Affective Picture System images}

The visual probe task consisted of three types of trials to explore whether attentional bias was due to a difficulty to disengage with smoking-related stimuli, or vigilance towards the smoking-related stimuli. These trials consisted of a probe replacing either neutral-neutral (NN), smoking-neutral (SN), or neutral-smoking (NS) picture pairs. The neutral-neutral images consisted of 40 images arranged into pairs from the International Affective Picture System (IAPS; Lang et al. 2008). The pictures were chosen to include a variety of scenes and avoid any drug-related connotations. These scenes included a lamp, palm trees, and a light bulb (the full picture list can be found in Appendix B). Each picture was originally validated by being rated on valence and arousal using a nine-point scale ranging from one (low valence or arousal) to nine (high valence or arousal). In the original validation documents, the 40 images used in this study had mean valence and arousal scores of $6.19(S D=1.37)$ and $3.87(S D=2.13)$ respectively. This indicates that the pictures were considered moderately pleasant and weakly arousing.

\section{Matched smoking and neutral images}

The images for smoking-neutral and neutral-smoking trials were created for this study and validated in a pilot study. Salience is relative for drug-related stimuli (Hester and 
Luijten 2013), and the internal consistency of the visual probe task has improved (at least for alcohol cues) when individualised drug-related stimuli are used (Christiansen et al. 2015). Considering this, different matched picture sets were created to include one for smokers who use roll-up cigarettes and one for smokers who use filter or manufactured cigarettes. The sets were limited to these two types of cigarettes as tobacco smokers were the population of interest, and manufactured and roll-up cigarettes are the most popular forms of tobacco product (West 2017). Twenty-five image sets were created which consisted of one neutral image (e.g. a fork against a mouth) and a closely matched smoking-related image (e.g. a cigarette against a mouth). The models used to take the images were a white male and white female smoker. The smokingrelated image featured either a roll-up or manufactured cigarette to produce a triplet of closely matched images for each set. Four male and fourteen female smokers who smoked an average of $16.28(S D=8.81)$ cigarettes per day rated both sets of the 25 smoking images. Similar to the pilot study conducted by Vollstädt-Klein et al. (2011), participants rated the images on a one (not at all) to ten (extremely) scale for arousal ("How arousing do you find this picture?"), craving ("How strong is the craving induced by this picture?"), and valence ("How pleasant do you find this picture?"). The 20 image sets containing the highest rated craving properties were included in the visual probe task. The content of these images contained two images of cigarette packaging, two images of a male and female smoker together, and eight images each of an individual male and female smoker in each set.

\section{Visual probe task}

The visual probe task used in this study was created using PsychoPy (version 1.83; Peirce 2009) and Figure 3.1 provides an overview of the trial structure. Each trial started with a fixation cross in the centre of the screen before two images were presented horizontally to the left and right of the cross (Price et al. 2015). Consistent with Chanon et al. (2010), the fixation cross remained on the screen throughout the trials to ensure its appearance did not compete for attention. The pictures remained on the screen for either $200 \mathrm{~ms}, 500 \mathrm{~ms}$, or $2000 \mathrm{~ms}$ depending on the SOA of the trial. At picture offset, a colon (:) was used as a probe and appeared in the location vacated by one of the images. The probe remained on the screen until the participant responded 
either left or right using the arrow keys. This type of response is consistent with the probe position version of the visual probe task which has been reported to be superior to the probe differentiation version (Salemink et al. 2008). After a response was made, there was an inter-trial interval randomised between $500 \mathrm{~ms}$ and $1500 \mathrm{~ms}$ before the next trial began.

The visual probe task consisted of 20 image pairs for neutral-neutral trials, and 20 image pairs for smoking-neutral and neutral-smoking trials. The order of the trials was pseudo-randomised to ensure a smoking-neutral or neutral-smoking pair always followed a neutral-neutral pair. Each picture pair was presented four times to cover each combination of image (left and right) and probe location (left and right). For each picture pair, this process was repeated for each SOA condition to result in a total of 480 trials: 240 neutral-neutral, 120 smoking-neutral, and 120 neutral-smoking trials. The task was divided into three blocks of 160 trials separated by a 30 second break to produce a task lasting approximately 20 minutes.

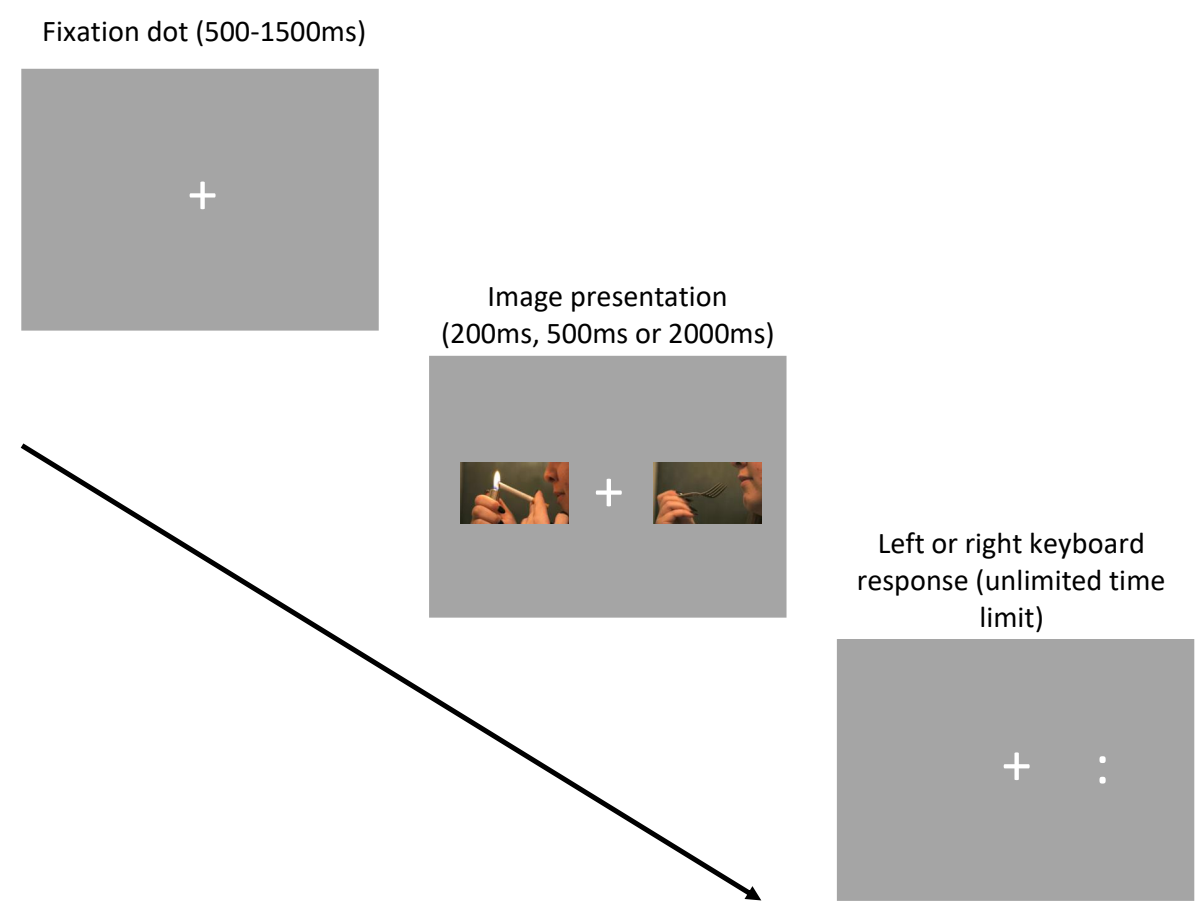

FIGURE 3.1: Overview of visual probe task used in Study One. This demonstrates a negative-smoking (NS) trial where the probe replaces the neutral image next to a smoking image. 


\subsubsection{Procedure}

At the start of each session, all participants were provided with an information sheet and informed consent form. This study was approved by the Faculty of Health and Life Sciences Ethical Approval board (Reference P37076, see Appendix C). All participants provided a measure of $\mathrm{CO}$ using a handheld monitor, before they completed online questionnaires to provide demographic information and a copy of the PANAS. Smoking participants completed additional copies of the QSU-brief, FTCD, and WISDM. The visual probe task was presented to participants on a desktop computer at a distance of $80 \mathrm{~cm}$ which was maintained throughout the experiment by the use of a chin rest. Smokers completed the visual probe task containing either the manufactured or roll-up images sets depending on their smoking preference. Non-smokers were randomly assigned either version of the task. Before starting the task, all participants received the same written instructions that outlined the length and procedure of the task. It began with 12 practice trials that provided feedback on the speed and accuracy of the response. The images used in the practice trials were additional images from the IAPS not used in the experimental trials. At the end of the practice trials, participants pressed a key to initiate the three blocks of experimental trials. At the end of the experiment, all participants completed another copy of the PANAS. Smokers also completed a second copy of the QSU-brief. Smokers completed the same picture rating task that was conducted in the picture pilot study to provide an additional measure of craving, valence, and arousal for the roll-up or manufactured cigarette image sets. All participants were provided with a debriefing letter outlining the aims and objectives of the study, and provided with the $£ 5$ store voucher if they chose this reimbursement option.

\subsubsection{Data analysis}

Data analysis was performed using R ( $R$ Core Team 2019). The R code for the results is available on the OSF project (https://osf.io/h9ex7/). The individual R packages used in each study are provided in Appendix C. Independent samples Welch's t-tests were used for variables that met parametric assumptions. This is because it provides 
better control over the type I error rate when the assumption of homogeneity of variance is violated (Delacre, Lakens and Leys 2017). For each ANOVA, the effect size generalised eta-squared $\left(\eta G^{2}\right)$ was used as between-subject and within-subject effects can be compared more easily than commonly used measured such as partial eta-squared (Bakeman 2005; Lakens 2013). Any within-subject factors that violated sphericity were adjusted using the Greenhouse-Geisser correction.

\subsubsection{Data reduction}

Incorrect responses on the visual probe task were removed from participant responses. The data were originally subjected to a data screening process similar to previous research using this task (e.g. Schoenmakers, Wiers, and Field 2008; Christiansen et al. 2015). This involved removing trials with responses outside $200 \mathrm{~ms}$ and $2000 \mathrm{~ms}$, and any responses outside three standard deviations of the mean. However, removing outliers using the mean and standard deviation approach is problematic (Ratcliff 1993). Therefore, this process was changed to any responses outside 2.5 times the median absolute deviation for each participant, SOA, and trial condition (Leys, Ley and Klein 2013). This process resulted in $7.82 \%$ of the total possible trials being removed, with the median number of excluded trials for each participant being 35 (range 12-87).

\subsubsection{Results}

\subsubsection{Demographic characteristics}

Table 3.2 shows the mean values for the participant demographic characteristics. Across the whole sample, participants were predominately white with the largest proportions being white British (45.00\%), white other (21.67\%), and white European (18.33\%). There was a smaller proportion of other (including uninformative responses such as Polish; $8.33 \%)$, black British or Caribbean (3.33\%), British Indian (1.67\%), and Indian/Pakistani (1.67\%). The key nicotine dependence measures are shown visually in Figure 3.2. 
TABLE 3.2: Study One mean (SD) values for participant characteristics and scale scores.

\begin{tabular}{lccc}
\hline Variable & Light smokers & Heavy smokers & Non-smokers \\
\hline Age & $21.14(3.55)$ & $24.11(8.12)$ & $23.15(5.63)$ \\
\hline CO & $3.59(3.11)$ & $10.61(7.41)$ & $0.25(0.55)$ \\
\hline $\begin{array}{l}\text { Gender } \\
\text { (female:male) }\end{array}$ & $17: 5$ & $10: 8$ & $10: 10$ \\
\hline $\begin{array}{l}\text { PANAS } \\
\text { positive before }\end{array}$ & $27.86(6.51)$ & $28.94(7.87)$ & $32.30(7.89)$ \\
\hline $\begin{array}{l}\text { PANAS } \\
\text { positive after }\end{array}$ & $25.14(6.94)$ & $26.17(8.99)$ & $30.20(11.11)$ \\
\hline $\begin{array}{l}\text { PANAS } \\
\text { negative before }\end{array}$ & $13.95(4.60)$ & $14.83(4.78)$ & $13.05(4.05)$ \\
\hline $\begin{array}{l}\text { PANAS } \\
\text { negative after }\end{array}$ & $14.14(4.48)$ & $12.72(3.14)$ & $12.70(3.96)$ \\
\hline FTCD & $1.00(0.82)$ & $3.89(0.89)$ & - \\
\hline CPD & $5.59(3.22)$ & $12.61(4.94)$ & - \\
\hline $\begin{array}{l}\text { WISDM total } \\
\text { Last cigarette } \\
\text { (minutes) }\end{array}$ & $47.74(10.93)$ & $47.96(11.16)$ & - \\
\hline Years as a smoker & $4.77(112.5)$ & $30.0(50.0)$ & - \\
\hline $\begin{array}{l}\text { QSU-B } \\
\text { factor 1 before }\end{array}$ & $17.68(8.44)$ & $20.00(8.22)$ & - \\
\hline $\begin{array}{l}\text { QSU-B } \\
\text { factor 1 after }\end{array}$ & $20.82(9.13)$ & $21.11(8.09)$ & - \\
\hline $\begin{array}{l}\text { QSU-B } \\
\text { factor 2 before }\end{array}$ & $12.23(6.94)$ & $11.39(6.27)$ & - \\
\hline $\begin{array}{l}\text { QSU-B } \\
\text { factor 2 after }\end{array}$ & $14.41(8.13)$ & $13.83(8.06)$ & - \\
\hline & & & \\
\hline
\end{tabular}

Notes and Abbreviations. CO (Carbon Monoxide); CPD (Cigarettes per day); FTCD (Fagerström Test for Cigarette Dependence); PANAS (Positive and Negative Affect Schedule); QSU-B (Brief Questionnaire of Smoking Urges); WISDM (Wisconsin Inventory for Smoking Dependence Motives). *Due to the large skew, these values represent the median and IQR.

\subsubsection{Control measures}

All participants completed the PANAS and all smokers completed the QSU-brief before and after completing the visual probe task. Mean values for each scale can be found in Table 3.2. A $3 \times 2$ mixed design ANOVA was performed on the PANAS data with one between-subjects IV of smoking group (heavy smoker, light smoker and non-smoker) and one within-subjects IV of time (pre and post visual probe task). A 2 x 2 mixed design ANOVA was performed on the QSU-brief data with one betweensubjects IV of smoking group (heavy smoker and light smoker) and one within-subjects IV of time (pre and post visual probe task). 

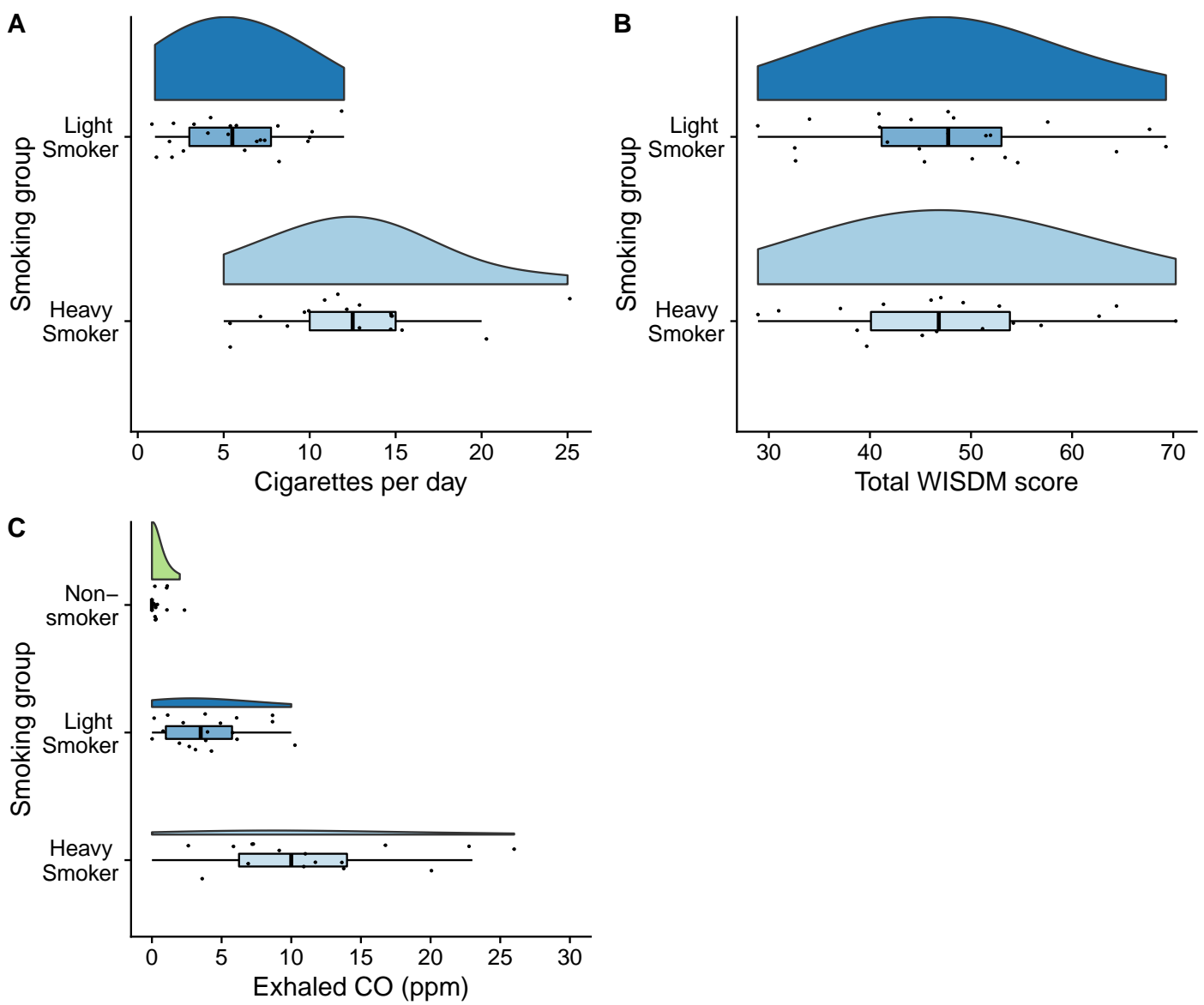

FIGURE 3.2: Three different measures of nicotine dependence: (A) number of cigarettes per day, (B) WISDM total score, and (C) exhaled concentration of $\mathrm{CO}(\mathrm{ppm})$. The data are presented as raincloud plots. The top element for each group represents the distribution of scores through the density. The bottom element presents the individual data points, with a boxplot superimposed to show summary statistics. Note the $\mathrm{x}$-axis of $\mathrm{B}$ ranges from $30-70$ due to the range of scores.

For the QSU-brief factor one, there was not a significant effect of smoking group $\left(\mathrm{F}(1,38)=0.29, p=.597, \eta G^{2}<.01\right)$, time $\left(\mathrm{F}(1,38)=3.56, p=.075, \eta G^{2}=.06\right)$, or an interaction $\left(\mathrm{F}(1,38)=0.76, p=.388, \eta G^{2}<.01\right)$. Similarly for the QSU-brief factor two, there was no significant effect of smoking group $\left(\mathrm{F}(1,38)=0.13, p=.725, \eta G^{2}<.01\right)$, time $\left(\mathrm{F}(1,38)=3.42, p=.072, \eta G^{2}=.06\right)$ or an interaction $\left(\mathrm{F}(1,38)=0.01, p=.917, \eta G^{2}\right.$ $<.01)$. This suggests that there was no substantial differences in the smoking groups reporting different levels of craving before or after completing the visual probe task.

For the positive affect sub-scale of the PANAS, there was a significant main effect of time $\left(\mathrm{F}(1,57)=12.38, p<.001, \eta G^{2}=.16\right)$. This shows that across all participants, there was a significant decrease in positive affect after completing the visual probe 
task (Cohen's $d=0.45,95 \% \mathrm{CI}=[0.20,0.73])$. On the other hand, there was no main effect of smoking group $\left(\mathrm{F}(2,57)=2.08, p=.134, \eta G^{2}=.04\right)$ or an interaction $(\mathrm{F}(2,57)$ $\left.=0.09, p=.913, \eta G^{2}<.01\right)$. For the negative affect sub-scale, there was no significant effect of smoking group $\left(\mathrm{F}(2,57)=0.54, p=.588, \eta G^{2}<.01\right)$, time $(\mathrm{F}(1,57)=2.51, p=$ $\left..119, \eta G^{2}=.02\right)$, or an interaction $\left(\mathrm{F}(2,57)=2.02, p=.142, \eta G^{2}=.03\right)$. This suggests that there was no substantial differences in negative affect before or after competing the visual probe task.

\subsubsection{Attentional bias}

Hypotheses

A significant interaction was expected between smoking group and trial type as light smokers were predicted to exhibit attentional bias specifically through vigilance. This would be indicated by faster reaction times to trials where the probe replaced smoking stimuli (smoking-neutral trials), than on trials where the probe replaced either neutral stimuli next to smoking stimuli (neutral-smoking trials) or two neutral stimuli presented together (neutral-neutral trials). Heavy smokers and non-smokers were not predicted to exhibit attentional bias to smoking stimuli. There was no a priori prediction for the three-way interaction between smoking group, SOA, and trial type.

\section{Confirmatory analyses}

Due to the large number of factors in a three-way ANOVA, the $p$ values were adjusted using the Holm correction (Holm 1979; Cramer et al. 2016). There was a significant main effect of trial $\left(\mathrm{F}(1.98,112.88)=11.38, p<.001, \eta G^{2}=.004\right)$. However, there was no main effect of SOA $\left(\mathrm{F}(1.77,100.73)=2.63, p=.334, \eta G^{2}=.004\right)$ or smoking group $\left(\mathrm{F}(2,57)=2.34, p=.334, \eta G^{2}=.07\right)$. This shows that across all participants, mean reaction time differed across trial conditions. However, in isolation this effect was not of interest. There was a significant interaction between trial and SOA $(F(3.70$, $\left.210.81)=22.55, p<.001, \eta G^{2}=.013\right)$, but not between smoking group and $\mathrm{SOA}(\mathrm{F}(3.53$, $\left.100.73)=1.54, p=.404, \eta G^{2}=.004\right)$. Importantly in regard to the hypotheses, there was no interaction between smoking group and trial $\left(\mathrm{F}(3.96,112.88)=2.57, p=.212, \eta G^{2}\right.$ $=.002)$, or a three-way interaction between smoking group, SOA, and trial $(\mathrm{F}(7.40$, $\left.210.81)=0.79, p=.605, \eta G^{2}=.001\right)$. The results are displayed graphically in Figure 
3.3. Light smokers were predicted to exhibit attentional bias towards smoking cues. However, contrary to predictions there was not a significant interaction to suggest that different smoking groups produced different responses towards different types of stimuli.

\subsubsection{Reliability}

Following the procedure of Christiansen et al. (2015), the internal consistency of the visual probe task was estimated by first creating an attentional bias score for each stimulus pair. This was calculated by subtracting the mean RT to smoking stimuli from the mean RT to neutral stimuli. This provides an attentional bias index for each stimulus pair in order to investigate whether participants respond similarly across the 20 stimulus pairs. This follows the procedure for determining whether responses to items within a scale is consistent. This procedure only involved the smoking participants as they completed either the roll-up or filter version of the visual probe task based on their self-reported smoking preferences. This indicated that in contrast to Christiansen et al. (2015), the internal consistency of the visual probe task in this study was poor despite using individualised smoking stimuli. This was the case for the 200ms $(\alpha=$ $.22,95 \% \mathrm{CI}=[.00, .42]), 500 \mathrm{~ms}(\alpha=.45,95 \% \mathrm{CI}=[.03, .67])$, and $2000 \mathrm{~ms}(\alpha=.42,95 \%$ $\mathrm{CI}=[.00, .63])$ SOA condition.

Calculating Cronbach's alpha has been reported for comparison with previous studies, but internal consistency may not be the best approach. Internal consistency measures assume the items, or in this case trials, are presented in the same order for each participant (Parsons, Kruijt, and Fox 2019). As cognitive tasks typically present stimuli in a randomised order, internal consistency may not be the best approach for reliability. A more suitable method is a permutation approach to calculating split-half reliability. This randomly splits the data set into two halves many times, and calculates the average correlation between each half for each condition. Using 5000 iterations, the reliability estimates using this method produced similarly suboptimal results for the $200 \mathrm{~ms}(r=.19,95 \% \mathrm{CI}=[-.21, .52]), 500 \mathrm{~ms}(r=.52,95 \% \mathrm{CI}=[.26, .73])$, and $2000 \mathrm{~ms}$ $(r=.36,95 \% \mathrm{CI}=[.04, .61]) \mathrm{SOA}$ condition. These estimates were corrected using the Spearman-Brown formula as splitting the data in half leads to underestimating the reliability (Parsons et al. 2019). Although thresholds for split-half reliability are not 
prescriptive, one guideline suggests estimates from .50 to .75 represent moderate reliability (Koo and Li 2016). This suggests the estimates here are suboptimal, with only the $500 \mathrm{~ms}$ condition in the lower boundary for moderate reliability.

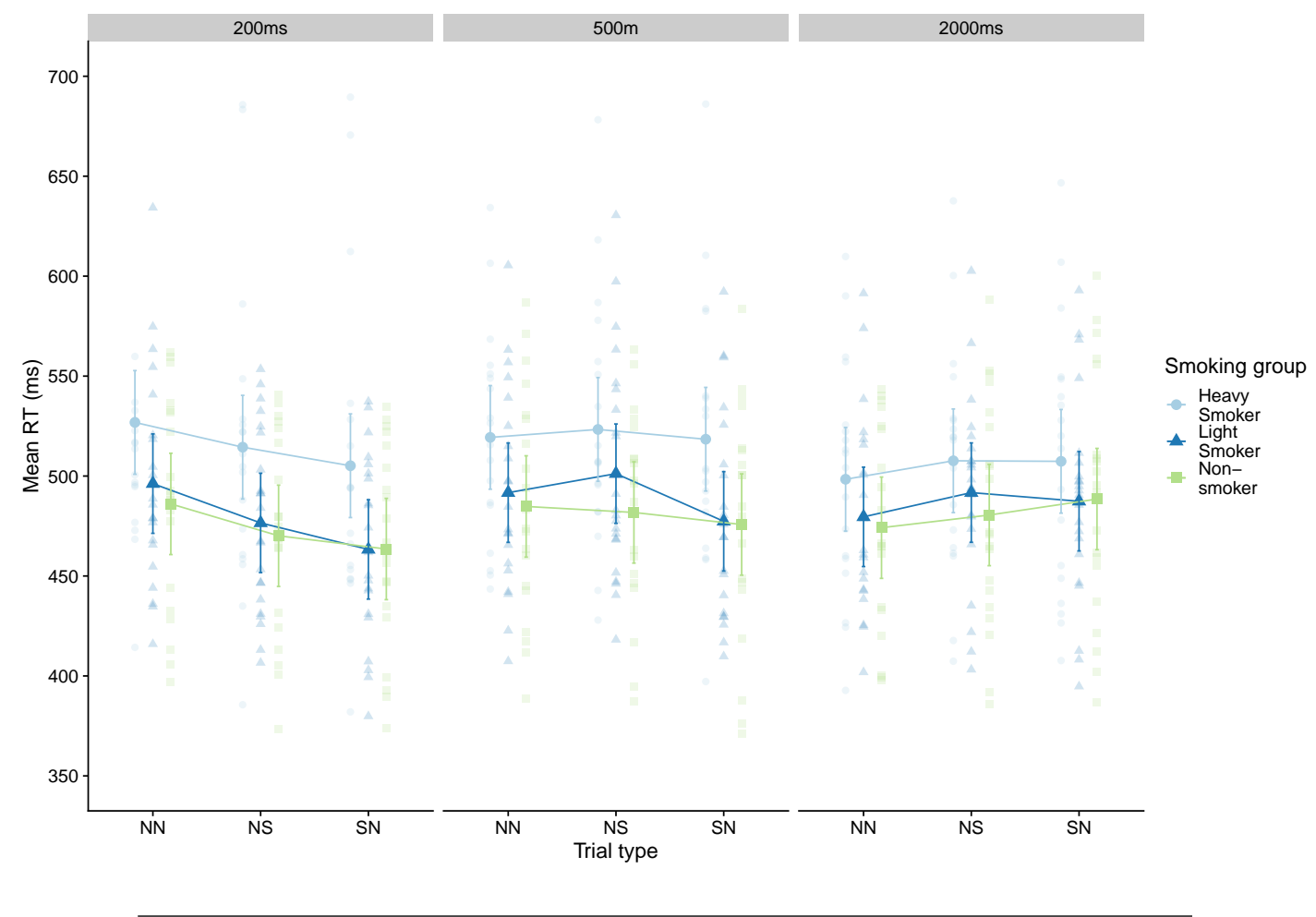

FIGURE 3.3: Interaction plot showing the mean attentional bias index across smoking group, SOA, and trial conditions. The error bars represent the $95 \% \mathrm{CI}$ around the mean. The individual data points are presented transparently in the background. $\mathrm{NN}$ = neutral-neutral trials; $\mathrm{NS}=$ neutral-smoking trials; $\mathrm{SN}=$ smoking-neutral trials.

\subsubsection{Picture ratings}

Table 3.3 shows the mean values for self-reported ratings of craving, valence, and arousal. A similar number of smokers in each group expressed a preference for roll-up and manufactured cigarettes with 11 light smokers completing each version, and 11 heavy smokers completing the manufactured version, and seven completing the rollup version. For each rating property, a 2 x 2 between-subjects ANOVA was performed with smoking group (heavy smokers and light smokers) and task version (roll-up and manufactured cigarettes) as between-group IVs. The DV was the mean rating across the image set. 
For craving, there was no main effect of smoking group $(\mathrm{F}(1,36)=0.80, p=.378$, $\left.\eta G^{2}<.001\right)$, task version $\left(\mathrm{F}(1,36)=0.09, p=.772, \eta G^{2}<.001\right)$, and no interaction effect $\left(\mathrm{F}(1,36)=0.23, p=.638, \eta G^{2}<.001\right)$. For valence, there was no main effect of smoking group $\left(\mathrm{F}(1,36)=0.06, p=.814, \eta G^{2}<.001\right)$, task version $\left(\mathrm{F}(1,36)=0.62, p=.436, \eta G^{2}\right.$ $<.001)$, and no interaction effect $\left(\mathrm{F}(1,36)=0.57, p=.455, \eta G^{2}<.001\right)$. Similarly, for arousal there was no main effect of smoking group $\left(\mathrm{F}(1,36)=0.05, p=.826, \eta G^{2}<\right.$ $.001)$, task version $\left(\mathrm{F}(1,36)=1.73, p=.197, \eta G^{2}=.019\right)$, and no interaction effect $(\mathrm{F}(1$, 36) $\left.=0.44, p=.513, \eta G^{2}<.001\right)$.

TABLE 3.3: Mean $(S D)$ values for each picture rating attribute for each version of the visual probe task.

\begin{tabular}{llcc}
\hline Attribute & Task type & Light smokers & Heavy smokers \\
\hline Craving & & & \\
& Roll-up & $5.80(3.02)$ & $4.49(3.96)$ \\
& Manufactured & $5.07(2.77)$ & $4.67(2.86)$ \\
\hline Valence & & & \\
& Roll-up & $5.28(2.69)$ & $4.35(3.67)$ \\
& Manufactured & $3.84(2.87)$ & $4.32(2.84)$ \\
\hline Arousal & & & \\
& Roll-up & $4.93(3.09)$ & $4.04(3.41)$ \\
& Manufactured & $2.92(3.11)$ & $3.37(3.18)$ \\
\hline
\end{tabular}

\subsubsection{Study One discussion}

In Study One, the results did not support the prediction that light smokers would show greater attentional bias towards smoking cues than heavy smokers. There was not a significant interaction effect between smoking group and trial type, nor a significant three-way interaction. This was contrary to previous attentional bias research using the visual probe task which has either shown evidence in favour of lighter smokers (Hogarth et al. 2003; Mogg et al. 2005) or heavier smokers (Vollstädt-Klein et al. 2011) exhibiting greater attentional bias towards smoking cues. Although the sample size of 20 participants per group was based on previous attentional bias research (e.g. Field et al. 2004), in hindsight this was not an optimal approach and likely led to the study being underpowered. Researchers commonly collect data based on rules of thumb, but this is often based on underestimating how many participants are required 
for a given effect (Bakker et al. 2016). Therefore, in order to test whether the lack of effect was truly a null result, or whether Study One was just not sensitive enough to detect an effect, Study Two was designed with statistical power in mind.

In order to sample a larger number of participants, data collection took place online where an increasingly large proportion of questionnaire-based research is conducted (Gosling and Mason 2015). Online samples provide access to a more diverse source of participants and in greater numbers than traditional undergraduate research participation schemes (Woods, Velasco and Levitan 2015). However, there has been scepticism about whether behavioural tasks that rely on accurately measuring response times can be conducted online. Several studies have tested whether web-based behavioural tasks can be used to replicate well-established effects in lab-based studies and tested the accuracy of the measurements. Using a range of tasks that rely on presenting stimuli and recording responses with millisecond accuracy, data collected from web-based samples are almost indistinguishable from lab-based samples (Germine, Nakayama and Duchaine 2012; Crump, McDonnell and Gureckis 2013; Hilbig 2016). Tasks ranging from the Stroop task to lexical decision making were successfully replicated. The limit appeared to be a task which required almost subliminal presentation of stimuli (64ms or less), as a priming effect with this presentation time did not replicate (Crump et al. 2013). However, this level of rapid presentation would require specialised hardware to be presented accurately even in lab-based studies. These studies suggest that behavioural tasks can be adequately conducted online.

Despite negligible consequences of conducting behaviour experiments online, there are still caveats to take into consideration. First, there is almost no control over the environment the participant completes the study in. When a participant completes a lab-based study, the researcher has full control over the environment, the number of distractions, and can at least make sure the participant looks like they are paying attention. Studies have found that a similar number of web-based and lab-based participants fail to understand the task instructions (Oppenheimer, Meyvis and Davidenko 2009; Crump et al. 2013; Clifford and Jerit 2014), showing that participant inattention is not necessarily a problem unique to online studies. However, online participants report substantially more distractions while they take part in studies, such as browsing the internet, listening to music, or watching TV (Clifford and Jerit 2014). Despite this, 
there was no association with performance on a manipulation check and the number of distractors. The effects of inattention can be countered by forcing participants to complete instructional manipulation checks until they demonstrate they understand the task instructions (Oppenheimer et al. 2009; Crump et al. 2013). Second, some studies found that the variance of responses is slightly higher in web-based samples (Hilbig 2016). In a simulation study, Brand and Bradley (2012) showed that even with increased technical variance from a range of hardware in online samples, the effect on statistical power is minimal. The worst case scenario resulted in a 3\% decrease in power, but this can be easily offset by the opportunity to sample more online participants in a shorter space of time. Third, as Study Two focused on smokers, their smoking habits are based entirely on self-report information and could not be verified using more objective measures like exhaled Carbon Monoxide. Ramo, Hall, and Prochaska (2011) showed that smoking-related information collected online displayed good reliability and validity, and correlated highly with a more in-depth timeline followback assessment. This shows that a lack of objective verification should be acknowledged as a limitation, but online estimates of smoking behaviour can still be valid and reliable. Taking into consideration the pros and cons of conducting a behavioural task online with smoking participants, the opportunity to sample far more participants than what is possible in a lab-based study outweighed the cons. Online samples are more diverse and representative of the population than traditional lab-based studies using undergraduate students (Woods et al. 2015). Moreover, providing that data quality is taken seriously, measures from web-based behavioural tasks are comparable to lab-based studies. Study Two recruited a larger online sample to address the limitation of low statistical power in Study One.

In addition to sample size, several other considerations were made to consolidate the design of Study Two. First, participants only completed one version of the visual probe task as opposed to being assigned one of two versions in Study One. The aim of including two versions of the task was to explore whether using individualised smoking stimuli improved internal consistency. This was based on Christiansen et al. (2015) as they demonstrated that individualised alcohol stimuli led to higher internal consistency than a task containing generic stimuli. In Study One, providing smoking participants with individualised smoking images still produced a task with poor 
levels of internal consistency. Using an alternative measure in the split-half method of reliability produced similarly sub-par results, with only the 500ms SOA condition reaching even the lower end of moderate consistency. Jones et al. (2018) reported further research into the ability to increase the internal consistency of the visual probe task. All their attempts failed to replicate the improved internal consistency estimates in Christiansen et al. (2015). This shows that there has been little success in improving the internal consistency of the visual probe task, even when more distinctive individualised alcohol stimuli were used. In Study Two, only one version of the visual probe task was used in order to focus on the comparison between smoking groups.

Second, the smoking groups in Study One were defined as light and heavy smokers using the FTCD with cut-off values used in previous research (e.g., Mogg et al. 2005; Vollstädt-Klein et al. 2011). The systematic review in Chapter Two demonstrated that the definition of different smoking groups is a contentious issue with a range of definitions used across studies such as light and heavy smokers, non-dependent and dependent smokers, and non-daily and daily smokers. Vollstädt-Klein et al. (2011) showed that differences in attentional bias between smoking groups were robust to different dichotomies. Although they sampled light smokers who smoked at least one cigarette per day, the authors did not report whether their sample included non-daily smokers. Nicotine dependence can be accurately viewed as a dichotomy between higher and lower dependence (Shiffman et al. 2012b), but differentiating between daily and non-daily smoking appears to be the most useful criteria (Husten 2009). It is the most stable across time, it can be ascertained using a small number of questions, and the distinction between daily and non-daily smoking is comparable to the questions used in large population studies.

Dichotomising the smokers using the FTCD in Study One also appeared to be problematic. There was a large amount of overlap between the two groups on other measures of nicotine dependence such as Carbon Monoxide, cigarettes per day, and the WISDM score. The individual data points in Figure 3.1 shows that some heavy smokers had a very low concentration of exhaled Carbon Monoxide which does not fit their smoking profile. The total WISDM score provides an additional self-report estimate of nicotine dependence through primary and secondary motives related to 
smoking behaviour (Piper et al. 2004). This measure demonstrated an almost identical distribution of scores to suggest splitting the groups based on FTCD did not capture the differences in the smoking behaviour effectively. In addition, both Study One and previous research (Heatherton et al. 1991; Haddock et al. 1999) reported less than adequate levels of internal consistency in the FTCD, which raises the question of whether it is a valid measure of nicotine dependence. Taking this into consideration, Study Two and the remainder of this thesis defined different smoking groups as daily and non-daily smokers as opposed to light and heavy smokers. This definition could not be used in Study One as the smokers did not complete questions relating to the number of days they smoked on.

There were two main aims of Study Two. The first was to investigate whether non-daily smokers showed greater attentional bias towards smoking cues than daily smokers. Study One failed to find an effect of smoking group, but as discussed previously, it is likely that this is explained by low statistical power. Therefore, Study Two aimed to investigate this by maximising statistical power and focusing on daily and non-daily smokers. In addition, a non-smoking control group was excluded in order to focus on the comparison of interest. Based on the direction of results of Study One and previous research, non-daily smokers were hypothesised to show greater attentional bias than daily smokers. The second aim was to investigate if attentional bias was greater in the initial orientation or maintenance of attention. Study One explored this using three different image presentation times. In Study Two, the design was consolidated to focus on the $200 \mathrm{~ms}$ and $500 \mathrm{~ms}$ SOA conditions, as these had the largest effect sizes. The protocol and hypotheses for this project were pre-registered on the OSF (https://osf.io/am9hd/). There was not an a priori hypothesis for the effect of SOA condition. Non-daily smokers were expected to show greater attentional bias than daily smokers, but it was not clear what the difference in magnitude would be under different SOA conditions. 


\subsection{Study Two}

\subsubsection{Method}

\subsubsection{Design}

Study Two used a $2 \times 2$ mixed quasi-experimental design as the smoking groups were self-selected. This consisted of one between-subjects IV of smoking group with two levels: daily and non-daily smokers. This was determined by the participants responding to the question "Do you usually smoke cigarettes every day?". Non-daily smokers were those who responded "No", and daily smokers were those who responded "Yes". In addition, there was a within-subjects IV of SOA of the visual probe task which had two levels: $200 \mathrm{~ms}$ and $500 \mathrm{~ms}$. The dependent variable was the attentional bias index (ms) calculated by subtracting the mean RT to smoking trials from the mean RT to neutral trials. Positive values indicate greater attentional bias towards smoking cues, and negative values indicate greater attentional bias towards neutral cues.

\subsubsection{Participants and sample size calculation}

In order to recruit a larger sample, data were collected online using the participant recruitment service Prolific (https://prolific.ac/). Participants were paid $£ 2$ for a study lasting 20 minutes. Inclusion criteria included all participants should have normal or corrected-normal vision, be between the ages of 18 to 60 , and smoke at least one cigarette per week or four cigarettes per month. Only smokers were included in this study in order to focus on comparing different smoking groups. The minimum smoking amount was low in order to detect non-daily smokers.

The sample size of Study Two was estimated by simulating a power analysis. The smallest effect size of interest was chosen based on estimates from Study One and previous research. In Study One, the mean difference in attentional bias score between the smoking groups was $6.13 \mathrm{~ms}(95 \% \mathrm{CI}=[-5.27,17.53])$ for a $200 \mathrm{~ms}$ SOA and $11.35 \mathrm{~ms}$ $(95 \% \mathrm{CI}=[-4.51,27.21]$ for a 500ms SOA. However, the confidence intervals around these estimates were wide due to the small sample size. Therefore, previous research in attentional bias was also consulted to investigate the smallest effects that have been 
reported. The smallest known effect size for a 200ms SOA was $5 \mathrm{~ms}$ (Chanon et al. 2009) and $11 \mathrm{~ms}$ for a 500ms SOA (Bradley et al. 2003). Taking these estimates into account, the smallest effect sizes of interest were $5 \mathrm{~ms}(200 \mathrm{~ms})$ and $10 \mathrm{~ms}(500 \mathrm{~ms})$. In addition to the effect size estimates, previous research was consulted to select a conservative estimate for the standard deviation of the mean difference. A standard deviation of 20ms around the attentional bias index in Vollstädt-Klein et al. (2011) appeared to be the largest estimate reported in previous attentional bias research. Together, the smallest effect sizes of interest were selected with the most conservative estimate for the standard deviation to estimate a sample size that would provide informative results.

These values were used to conduct a simulated power analysis for a $2 \times 2$ mixed ANOVA using $\mathrm{R}$. The code for the power analysis can be found in the pre-registration protocol for Study Two (https://osf.io/am9hd/). Based on the direction of results in Study One, and previous research on smoking motives, non-daily smokers were expected to display greater attentional bias towards smoking cues than daily smokers. The conditions of the power analysis were set as non-daily smokers having a $5 \mathrm{~ms}$ (200ms) and 10ms (500ms) greater mean difference in attentional bias score than daily smokers. For each condition, the values were sampled from a normal distribution with a conservative standard deviation of $20 \mathrm{~ms}$. The sample size for each smoking group was increased from $10(\mathrm{~N}=20)$ to $150(\mathrm{~N}=300)$ in steps of 10 . For each step, the procedure was repeated 10,000 times in a Monte Carlo simulation procedure. Alpha was set as .05 and beta was set as .20. Eighty percent power was reached between 50 and 60 participants per group. The target for the final sample size was 60 per group $(\mathrm{N}=120)$ to avoid underestimating power.

\subsubsection{Materials}

Fagerström Test for Cigarette Dependence (FTCD)

The FTCD (Heatherton et al. 1991; Fagerström 2011) was used as a self-report questionnaire of nicotine dependence. This was not used to classify the smokers, but to provide a comparative measure to previous research that reported FTCD scores. The internal consistency of the FTCD in Study Two was higher than in Study One and estimates reported in previous literature $(\alpha=.74,95 \% \mathrm{CI}=[.67, .79])$. 


\section{Visual probe task and images}

The images used in the visual probe task were the same as those used in Study One. However, four image pairs were removed because they contained branded cigarette packaging. In May 2017, cigarettes in the United Kingdom had to be sold in plain packaging (Moodie et al. 2018). As the images predated May 2017, cigarette packaging was still branded. Recent research has shown that smokers fixate on different areas of cigarette packaging depending on whether branded or plain packaging is shown (Retzler, Shiraj, and Retzler 2019). Therefore, images where branded packaging could be seen were removed. This left 16 smoking and matching neutral image pairs. Similarly, the same images from the IAPS (Lang et al. 2008) used in Study One were included, but reduced to maintain the number of picture pairs at 16. As Study One and repeated attempts in other research (Jones et al. 2018) have not found an increase in reliability when individualised stimuli are used, only one set of images were used.

The visual probe task used in this study was created using Gorilla (Anwyl-Irvine et al. 2019) and Figure 3.4 presents an overview of the trial structure. This is an online platform that presents behavioural tasks in the participant's browser. Gorilla was integrated with Prolific and each participant was directed to complete the task in a new browser window. Each trial started with a fixation cross in the centre of the screen. This screen was presented for $250 \mathrm{~ms}$ before two images were presented horizontally to the left and right of the cross. The fixation cross remained on the screen to ensure its appearance did not compete for attention. The pictures remained on the screen for either $200 \mathrm{~ms}$ or $500 \mathrm{~ms}$ depending on the SOA condition. When the pictures disappeared, a small dot was used as a probe and appeared in the location vacated by one of the images. The probe remained on the screen until the participant responded either left ( $Z$ key) or right ( $M$ key). This was a probe position version of the visual probe task. After a response was made, the screen containing only the fixation cross appeared to signal the start of the next trial.

The task consisted of 16 image pairs for neutral trials, and 16 image pairs for smoking and matching non-smoking trials. The order of the trials was randomised for each participant. Each picture pair was presented four times to cover each combination of image (left and right) and probe location (left and right). For each picture pair, this process was repeated for each SOA condition. To create a sufficient number of trials in 
each condition, this procedure was repeated twice and presented in two blocks. There were 384 trials overall with 64 trials in each SOA and trial type condition. Due to a coding error, there was not a $500 \mathrm{~ms}$ neutral condition, as the $200 \mathrm{~ms}$ neutral condition was duplicated. Therefore, the distinction between vigilance and disengagement could unfortunately not be fully explored as in Study One. Exploratory analyses are presented in the results comparing different attentional bias indexes for the $200 \mathrm{~ms}$ SOA condition.

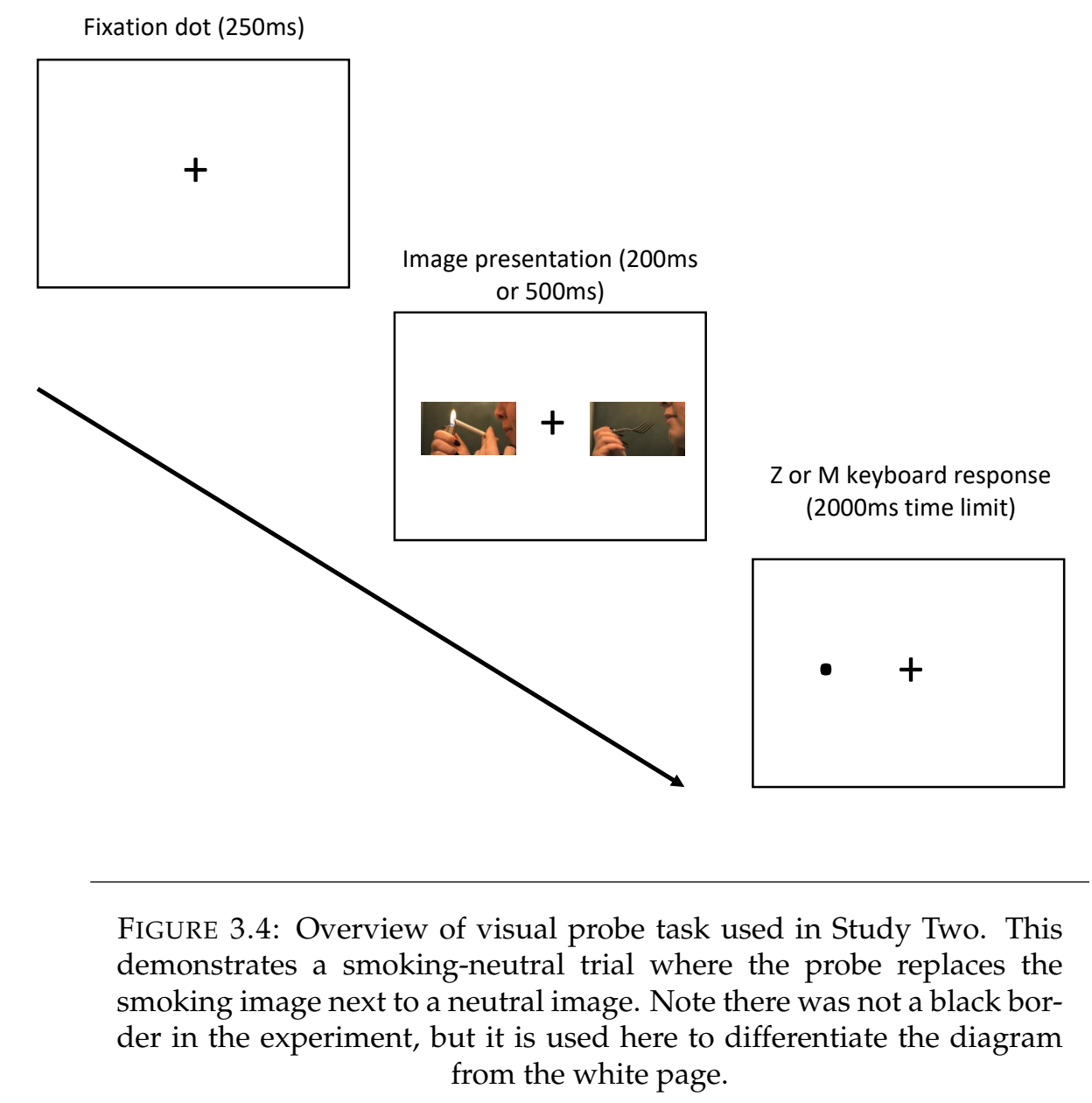

\subsubsection{Procedure}

When each participant opened the experiment, they were provided with an information sheet and provided informed consent by ticking a box. This study was approved by the Faculty of Health and Life Sciences Ethical Approval board (Reference P88261, see Appendix C). Participants completed a short questionnaire on their demographic information, smoking habits, and a version of the FTCD. The next page contained the 
visual probe task. This began with a set of instructions and asked the participant to complete the task in a quiet environment free of distractions. They then completed 12 practice trials which provided feedback on their responses and overall accuracy. The practice trials contained additional images from the IAPS which were not included in the experimental trials. The two experimental blocks were divided with a break screen that allowed the participant to start the second block as soon as they were ready. When the task was complete, participants completed four questions on their experience completing the study. These included whether they experienced any technical issues, whether they used an ineligible device, and if they had completed the study before. Similar to Clifford and Jerit (2014), the participants were also asked if they had any distractions while they completed the study such as listening to music or talking to someone. Participants were then provided with a debrief before they were redirected to Prolific. If the participants successfully reached the end of the study, they were paid $£ 2$ for their time.

\subsubsection{Data analysis}

Data analysis was performed using $\mathrm{R}$. The individual $\mathrm{R}$ packages are reported in Appendix $\mathrm{C}$. The $\mathrm{R}$ code for the results is available on the OSF project (https://osf .io/ am9hd/). Independent samples Welch's t-tests or ANOVA were used for variables that met parametric assumptions. For variables that did not meet parametric assumptions, non-parametric tests were performed where appropriate. For each ANOVA, the effect size generalised eta-squared $\left(\eta G^{2}\right)$ was reported.

\subsubsection{Data reduction}

Incorrect responses on the visual probe task were removed from participant responses. Responses faster than $200 \mathrm{~ms}$ were removed due to being preemptive responses. Outliers were defined as any responses outside 2.5 times the median absolute deviation for each participant, SOA, and trial condition (Leys et al. 2013). This process resulted in $9.72 \%$ of the total possible trials being removed, with the median number of excluded trials for each participant being 23 (range 7 - 98). 


\subsubsection{Results}

\subsubsection{Demographic characteristics}

From a sample of 218 people who accessed the study on Gorilla, 205 fully completed the experiment and received payment. After applying exclusion criteria, the final sample was 166, with 60 non-daily and 106 daily smokers. Participants were coded as being included if they did not violate any of the exclusion criteria. The reasons for the 39 participants being excluded were having fewer than $50 \%$ of the possible trials $(n=$ 4), experienced technical issues while completing the task $(n=16)$, incompatible participants who reported to smoke every day but not every week $(\mathrm{n}=3)$, and smokers who had not smoked in the past four weeks $(n=19)$. The total number equals 42 as some participants met more than one exclusion criterion.

The final sample size of 166 is highlighted as a deviation from the pre-registration protocol as the original plan was to sample 60 participants in both groups. The study advert on Prolific was originally aimed at frequent smokers, with a filter for daily smokers reporting to smoke at least one cigarette per day. When 60 daily smokers were recruited, the filter was changed to non-daily smokers reporting to smoke either one to six times per week, or less than four times per month. However, daily smokers still took part in the study despite the filter being changed, which led to oversampling.

The key demographic information for each smoking group is presented in Table 3.4. Consistent with their profile, daily smokers smoked more cigarettes per day and had a higher FTCD score. Non-daily smokers exemplified infrequent smoking as the median time since their last cigarette was 48 hours, while it was only one hour for daily smokers. Figure 3.5 shows the distribution of FTCD and cigarettes per day for both groups. The majority of non-daily smokers report low values, while daily smokers are distributed more evenly across both measures.

Non-daily smokers came from 18 different countries of origin, with the five most common being the United Kingdom (18: 30\%), Poland (9: 15\%), Italy (6: 10\%), Portugal (5: 8\%), and Estonia (3: 5\%). Daily smokers came from 26 different countries of origin, with the five most popular being the United Kingdom (24: 23\%), Poland (17: 16\%), Italy (12: 11\%), Portugal (8: 8\%), and Spain (7: 7\%).

In non-daily smokers, the percentages for the level of education were graduated 
TABLE 3.4: Study Two mean (SD) values for participant characteristics and scale scores.

\begin{tabular}{lcc}
\hline & Non-daily smokers & Daily smokers \\
\hline Age & $28.68(7.71)$ & $31.84(9.70)$ \\
\hline $\begin{array}{l}\text { Gender } \\
\text { (female:male) }\end{array}$ & $23: 37$ & $48: 58$ \\
\hline White (\%) & $93 \%$ & $92 \%$ \\
\hline FTCD & $0.52(1.31)$ & $2.58(2.17)$ \\
\hline CPD & $2.38(2.74)$ & $8.59(6.41)$ \\
\hline Age started to smoke & $18.51(3.65)$ & $17.93(3.47)$ \\
\hline $\begin{array}{l}\text { Time since last cigarette } \\
\text { (minutes) }\end{array}$ & $2880(4590)$ & $60(633.75)$ \\
\hline
\end{tabular}

Note. *Due to the large skew, these values represent the median and IQR.

university (17: $28 \%$ ), had some university education (16: $27 \%)$, had a postgraduate degree (14: 23\%), graduated secondary school (11: 18\%), and had less than secondary school education (2: 3\%). In daily smokers, the percentages for the level of education were graduated university (35: 33\%), graduated secondary school (28: 26\%), had some university education (24: $23 \%$ ), had a postgraduate degree (16: 15\%), and had less than secondary school education (3: 3\%).

\subsubsection{Attentional bias}

\section{Confirmatory analyses}

The mean (SD) attentional bias index in the $200 \mathrm{~ms}$ SOA condition was $1.95 \mathrm{~ms}(22.31)$ for daily smokers, and -0.30ms (18.57) for non-daily smokers. In the 500ms SOA condition, the mean bias score was $0.21 \mathrm{~ms}$ (21.93) for daily smokers, and -2.06ms (12.67) in non-daily smokers. This was in the opposite direction to the predicted effect, as non-daily smokers were expected to display a $5 \mathrm{~ms}$ bias index in the $200 \mathrm{~ms}$ condition, and $10 \mathrm{~ms}$ bias index in the $500 \mathrm{~ms}$. The results are displayed graphically in Figure 3.6.

Levene's test suggested homogeneity of variance was upheld for the 200ms SOA condition $(\mathrm{F}(1,164)=0.97, p=.325)$, but not for the 500ms SOA condition $(\mathrm{F}(1,164)$ $=4.54, p=.035)$. Q-Q plots suggested there may be some minor deviations with normality. Therefore, the results are reported as a parametric $2 \times 2$ mixed ANOVA for consistency with the pre-registration protocol. Two non-parametric Mann-Whitney U 

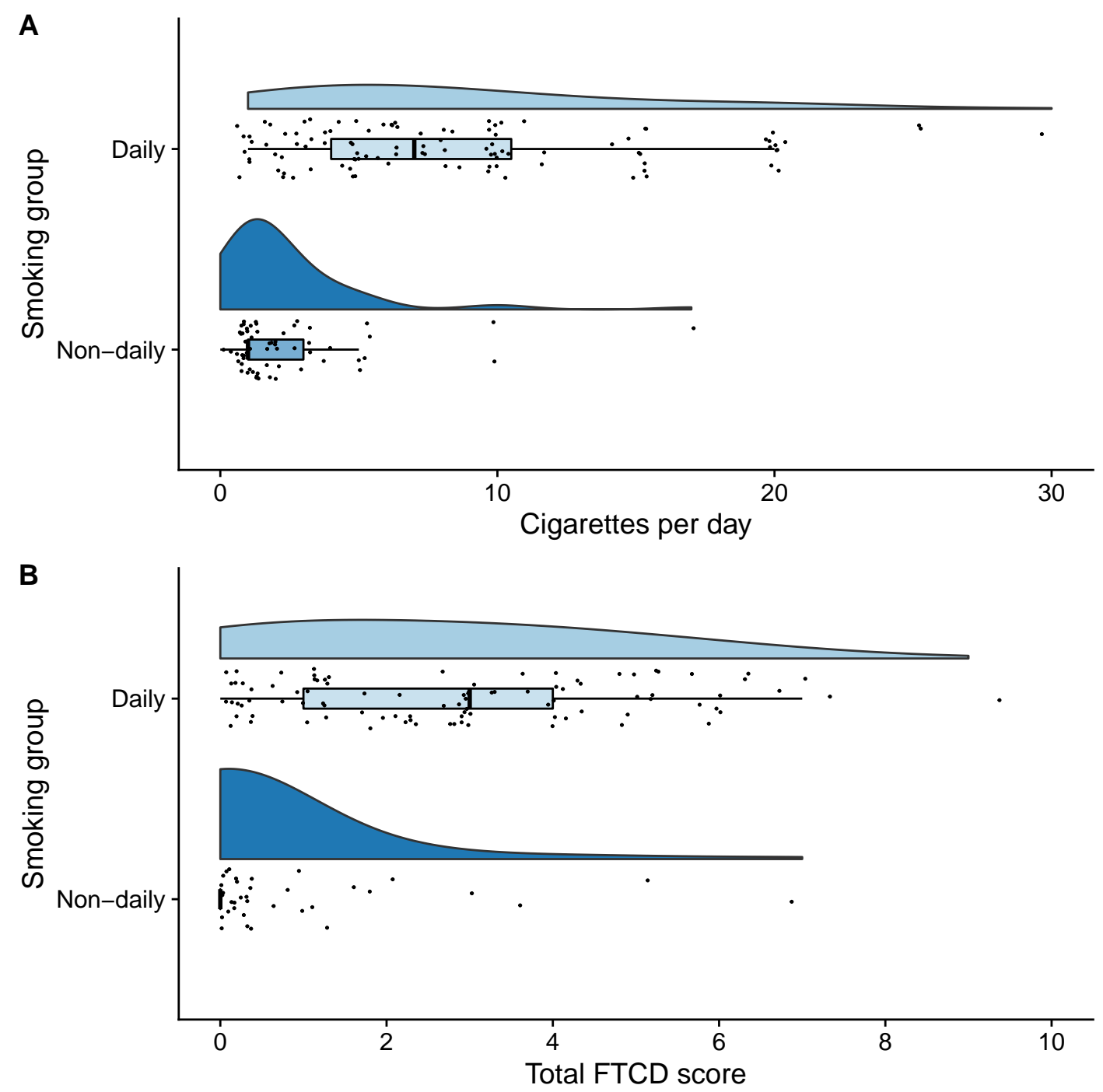

FIGURE 3.5: Two different measures of nicotine dependence: (a) number of cigarettes per day, and (b) FTCD score. The data are presented as raincloud plots. The top element for each group represents the distribution of scores through the density. The bottom element presents the individual data points, with a boxplot superimposed to show summary statistics.

tests are then presented as exploratory analyses to ensure the results are robust to the choice of parametric or non-parametric test.

A mixed ANOVA was conducted with SOA as a within subjects IV and smoking group as a between subjects IV. The mean attentional bias index was used as the DV. There was not a significant effect of SOA $\left(F(1,164)=0.58, p=.448, \eta G^{2}=.002\right)$ or smoking group $\left(\mathrm{F}(1,164)=0.97, p=.325, \eta G^{2}=.003\right)$. There was also no significant interaction between the two factors, $\mathrm{F}(1,164)<.001, p=.996, \eta G^{2}<.001$. Figure 3.6 demonstrates that the confidence intervals around the mean attentional bias index 
cross zero. This suggests that in addition to the smoking groups not being significantly different to each other, none of the groups or conditions expressed attentional bias towards smoking cues. None of these differences approached the smallest effect sizes of interest for each SOA condition. These findings did not support the pre-registered prediction that non-daily smokers would show greater attentional bias towards smoking cues than daily smokers.

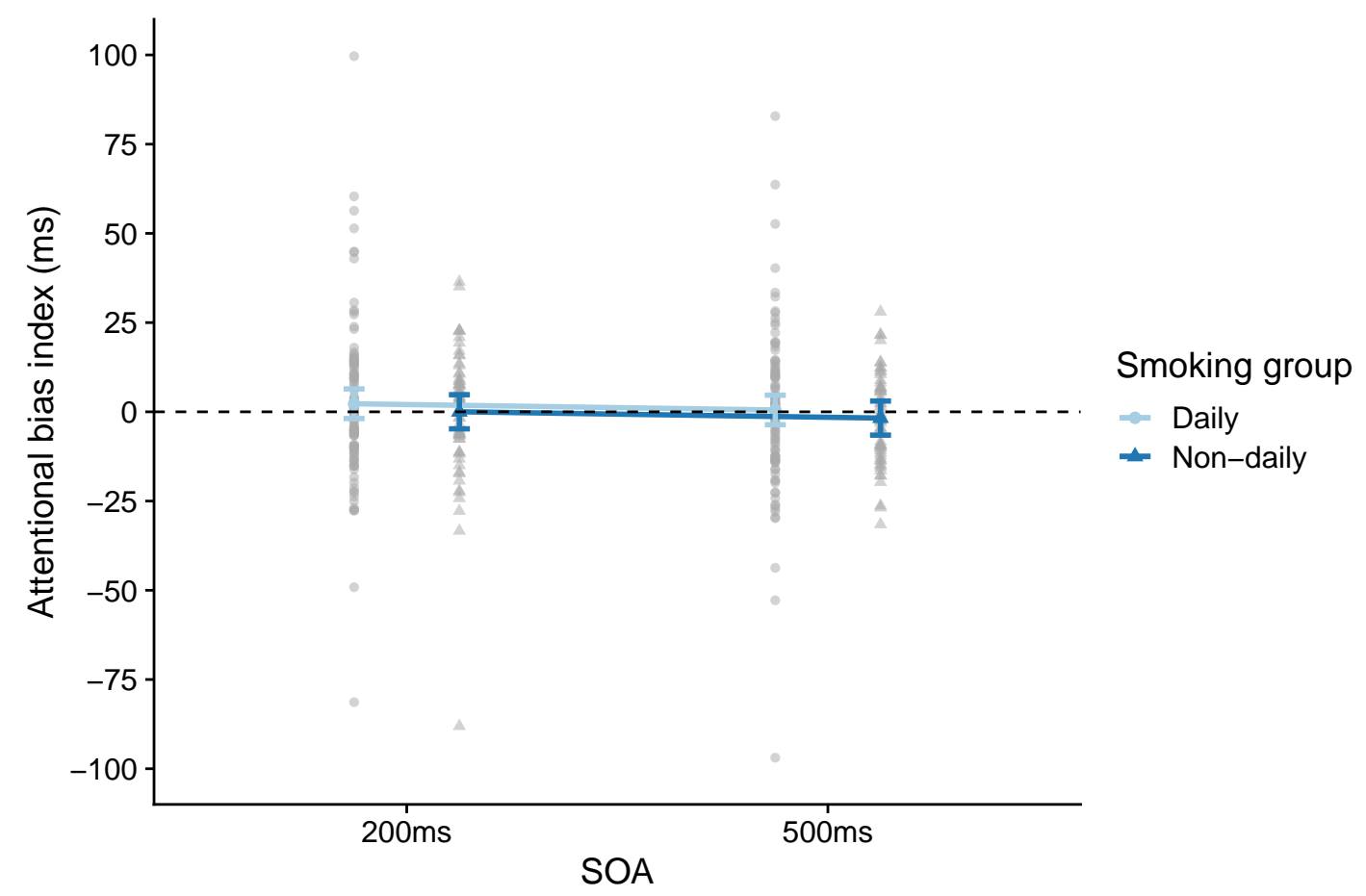

FIGURE 3.6: Interaction plot showing the mean attentional bias index for daily and non-daily smokers by SOA condition. The error bars represent the 95\% CI around the mean. Positive values indicate greater attentional bias towards smoking cues. Negative values indicate greater attentional bias towards neutral cues. The grey points show the individual scores per condition. 
Exploratory analyses: follow-up non-parametric tests

The following analyses are listed as exploratory as they were not specified in the preregistration protocol. As there were some minor violations of parametric assumptions, two Mann-Whitney U tests, one for each SOA condition, were performed in place of the $2 \times 2$ mixed ANOVA. There was not a significant difference between the two smoking groups for either the 200ms $(\mathrm{U}=3187, p=.983, r=.002,95 \% \mathrm{CI}=[-.179, .183])$ or 500ms $(\mathrm{U}=2937, p=.415, r=-.076,95 \% \mathrm{CI}=[-.254, .106])$ SOA condition. This shows that the non-significant effects were robust to the choice of parametric or nonparametric tests.

\section{Exploratory analyses: different smoking group definitions}

One of the issues highlighted in the systematic review in Chapter Two was the heterogeneity in smoking labels and definitions. The distinction between daily and nondaily smoking appears to have the most support, but one of the reasons there may have been no effect is it was sensitive to the choice of criteria used. Daily and nondaily smoking had not previously been used in attentional bias research. Therefore, exploratory analyses were performed to explore whether there was an effect when smokers were dichotomised using the number of cigarettes per day or their FTCD score. These analyses were performed on 163 participants, as there were technical issues on the number of cigarettes per day reported by three smokers. The entry was a date rather than the number of cigarettes per day, meaning the participant's computer potentially auto-filled the box based on a previously completed form.

First, smokers were categorised using the number of cigarettes per day. Light smokers $(n=118)$ were defined as those smoking fewer than ten cigarettes per day, and heavy smokers $(n=45)$ smoked ten or more cigarettes per day. The interaction plot for this analysis is shown in Figure 3.7. There was not a significant effect of SOA $\left(\mathrm{F}(1,161)=0.80, p=.372, \eta G^{2}=.002\right)$ or smoking group $\left(\mathrm{F}(1,161)=0.12, p=.728, \eta G^{2}\right.$ $<.001)$. There was also no significant interaction effect between the two factors, F (1, 161) $=0.06, p=.808, \eta G^{2}<.001$.

Second, smokers were categorised using FTCD scores. Non-dependent smokers ( $n=107)$ were defined as those with scores of two or fewer, and dependent smokers $(n=56)$ had scores of three or more. The interaction plot for this analysis is shown in 


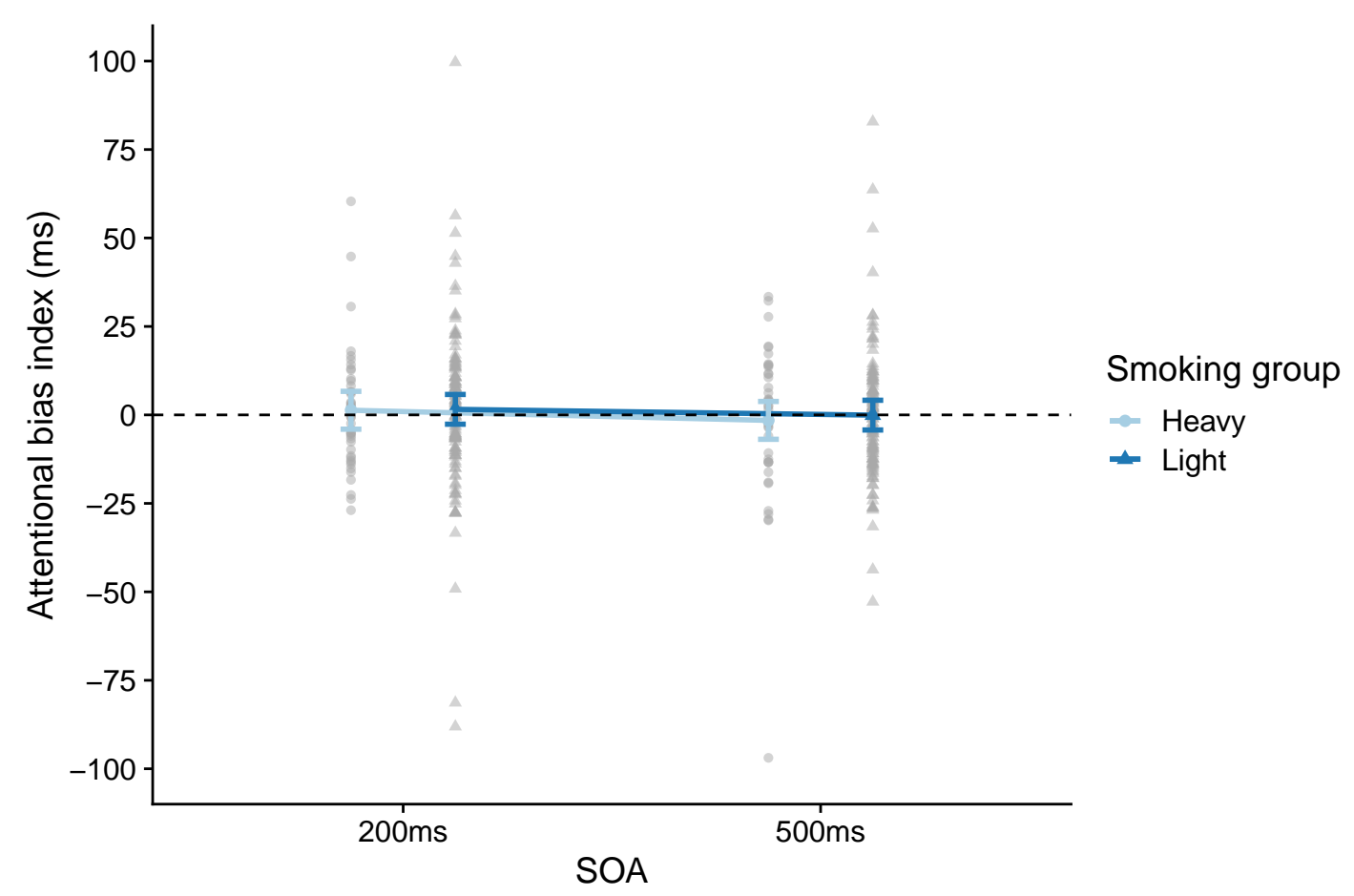

FIGURE 3.7: Interaction plot showing the mean attentional bias index for exploratory analyses when smokers are reclassified by the number of cigarettes they smoke per day. The error bars represent the $95 \% \mathrm{CI}$ around the mean. Positive values indicate greater attentional bias towards smoking cues. Negative values indicate greater attentional bias towards neutral cues. The grey points show the individual scores per condition.

Figure 3.8. There was not a significant effect of SOA $\left(F(1,161)=0.79, p=.376, \eta G^{2}\right.$ $=.002)$ or smoking group $\left(\mathrm{F}(1,161)=0.19, p=.661, \eta G^{2}=.001\right)$. There was also no significant interaction effect between the two factors, $\mathrm{F}(1,161)=0.03, p=.865, \eta G^{2}<$ .001 .

Exploratory analyses: orienting versus disengaging attentional bias index

Due to a coding error, the distinction between orienting and disengaging could not be explored as in Study One as there was no 500ms SOA condition for the neutral condition. Therefore, the $200 \mathrm{~ms}$ SOA condition was explored to compare the orienting and disengaging index in daily and non-daily smokers. Two Welch's t-tests were performed with smoking group as the IV and the attentional bias index (orienting or 


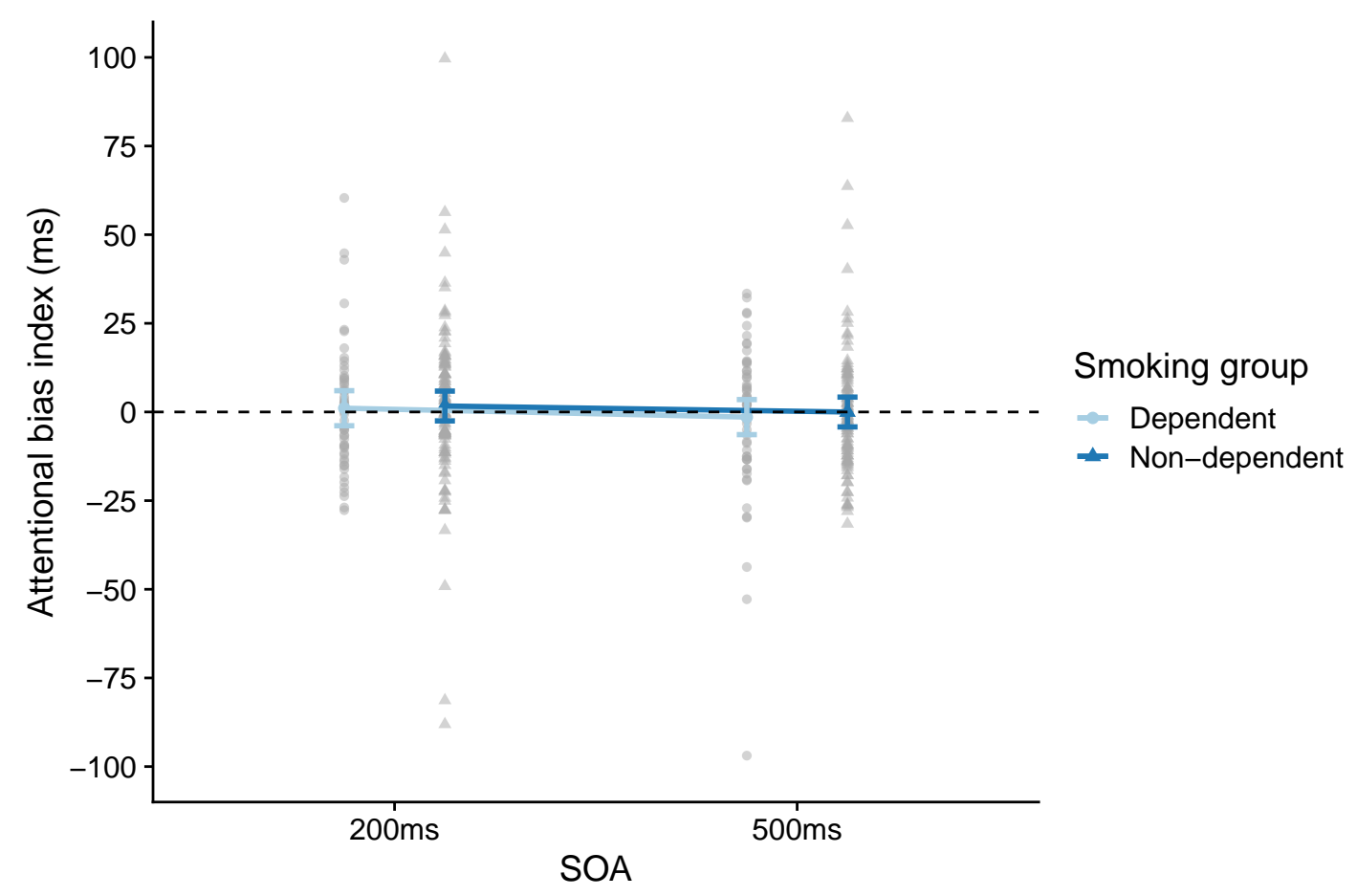

FIGURE 3.8: Interaction plot showing the mean attentional bias index for exploratory analyses when smokers are reclassified by their FTCD score. The error bars represent the $95 \% \mathrm{CI}$ around the mean. Positive values indicate greater attentional bias towards smoking cues. Negative values indicate greater attentional bias towards neutral cues. The grey points show the individual scores per condition.

disengaging) as the DV. Figure 3.9 shows the traditional attentional bias index presented next to the orienting and disengaging index.

The orienting index was created by subtracting the mean response time to smoking trials (smoking images presented next to non-smoking images) from the mean response time to neutral trials (two neutral images). Positive values mean there was greater attentional bias towards smoking images as responses were faster than neutral trials. Daily smokers $(M=5.59, S D=22.25)$ had a higher orienting index than nondaily smokers $(M=4.56, S D=16.52)$, but there was no significant difference with a negligible effect size, $t(152.19)=0.34, p=.736$, Cohen's $\mathrm{d}=0.05,95 \% \mathrm{CI}=[-0.27,0.37]$.

The disengaging index was created by subtracting the mean response time to neutral trials from the mean response time to non-smoking trials (non-smoking images presented next to smoking images). Positive values here mean responses were slower when probes replaced non-smoking images due to smoking images being distracting 
and delaying responses. Daily smokers $(M=-7.54, S D=22.89)$ had a lower disengaging index than non-daily smokers $(M=-4.27, S D=13.64)$, but there was no significant difference and a small effect size, $t(163.52)=-1.15, p=.250$, Cohen's $\mathrm{d}=-0.16,95 \% \mathrm{CI}$ $=[-0.48,0.16]$.

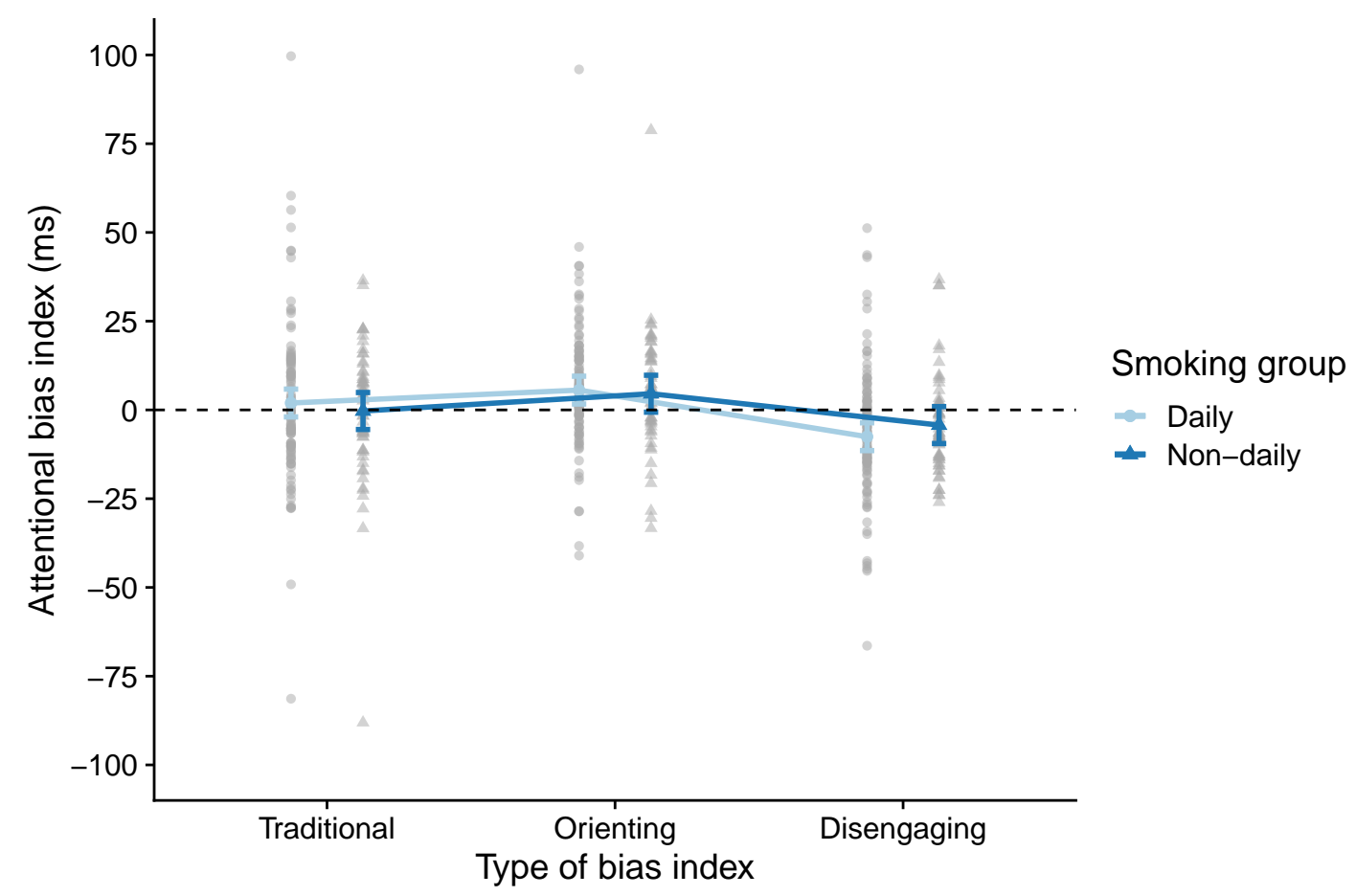

FIGURE 3.9: Interaction plot showing the mean attentional bias for traditional, orienting, and disengaging indexes (200ms SOA only). Positive values for the traditional index indicate faster responses towards smoking cues than non-smoking cues. Positive values for the orienting index mean faster responses towards smoking images in comparison to neutral trials. Positive values for the disengaging index mean slower responses towards non-smoking images in comparison to neutral trials. The error bars represent the $95 \% \mathrm{CI}$ around the mean. The grey points show the individual scores per condition.

Exploratory analyses: Bayesian test to explore null results

The a priori power analysis specifically focused on the main effect for smoking groups, as non-daily smokers were expected to show a consistent positive effect size over daily smokers. However, the mixed ANOVA and non-parametric tests produced nonsignificant $p$ values which cannot be used to support the null hypothesis (Greenland et al. 2016). Therefore, a Bayesian version of the mixed $2 \times 2$ ANOVA was performed 
as it can provide evidence for a null model over alternative models.

For a brief overview of Bayesian statistics, it is based on an alternate theory of probability as the frequentist statistics that $p$ values are based on (Dienes 2008). Frequentist statistics assign probability through long-run behaviour or procedures. The aim of this branch of statistics is to help make decisions and limit the number of type I and type II errors made in the long-run (Neyman 1977). A non-significant $p$ value does not mean the null hypothesis should be accepted, just that the data are not surprising assuming the null hypothesis is true. It is the probability of the data given a hypothesis, as opposed to the probability of a hypothesis given data. On the other hand, Bayesian statistics are based on a subjective theory of probability where probability can be assigned to specific events, rather than only long-run procedures (Dienes 2008). This can be applied to hypothesis testing where the probability of one hypothesis in relation to a second hypothesis can be calculated, providing the probability of a hypothesis given data. For example, the probability of the null hypothesis relative to an alternative hypothesis. This provides the Bayes factor which is a ratio of the probability of one hypothesis given the observed data, relative to another hypothesis. A Bayes factor of one means there is equal support for both hypotheses, and more data are required. A Bayes factor of five would mean one hypothesis is five times more likely given the data than the other hypothesis. There are no clear cut thresholds in Bayesian statistics, but a Bayes factor of three or more is taken as moderate evidence of one hypothesis relative to another, and ten or more is taken as strong evidence (van Doorn et al. 2019).

A Bayesian equivalent of the $2 \times 2$ mixed ANOVA was performed using JASP (JASP Team 2019). A default prior probability distribution (referred to from now as the prior) was used with an $r$ scale of 0.5 for fixed effects and 1.0 for random effects. A prior is important in Bayesian statistics as it informs the model of any expectations before seeing any data and constrains the model parameters. A default prior for an ANOVA model is helpful computationally and applicable across a wide range of scenarios (Rouder et al. 2012). The model results were compared to a null model which stated the results can just be explained through the individual variance of each participant. The null model was the best model, with a Bayes factor in support of $\mathrm{BF}_{m}$ over equal odds for each effect, $\mathrm{BF}_{m}=9.62$. This indicates moderate to strong support, 
with the null model explaining the data 9.62 times better than equal odds assigned to each effect. The pre-registration protocol outlined two effects of interest: the main effect of smoking group, and the interaction between smoking group and SOA. For both effects, there was evidence in favour of the null model. There was moderate evidence for the null model over the smoking group effect, $\mathrm{BF}_{01}=4.76$ (error percentage $=1.07 \%)$. There was strong evidence for the null model over the smoking group and SOA interaction effect, $\mathrm{BF}_{01}=161.24$ (error percentage, $3.03 \%$ ). This demonstrates support for the null model over both pre-registered effects of interest.

\subsubsection{Reliability}

Similar to Study One, the internal consistency of the visual probe task in this study was poor for both the $200 \mathrm{~ms}(\alpha=.28,95 \% \mathrm{CI}=[.00, .58])$ and $500 \mathrm{~ms}(\alpha=.19,95 \%$ $\mathrm{CI}=[.00, .43]) \mathrm{SOA}$ condition. This was also reflected in the permutation based splithalf estimate (corrected using the Spearman-Brown formula) with suboptimal results for the $200 \mathrm{~ms}(r=.48,95 \% \mathrm{CI}=[.35, .60])$ and $500 \mathrm{~ms}(r=.36,95 \% \mathrm{CI}=[.21, .50]) \mathrm{SOA}$ condition. This suggests the estimates here were suboptimal even for the lower bound of moderate reliability.

\subsubsection{Study Two discussion}

The aims of Study Two were to determine whether non-daily smokers would show greater attentional bias to smoking cues than daily smokers, and investigate whether attentional bias would be present in the initial orientation or maintenance of attention. In order to address the limitations of Study One, the design was consolidated to focus on smokers and two SOA conditions. The sample size was determined using a simulated power analysis to address the limitation of low statistical power. Despite these changes, there was not a significant difference in attentional bias between daily and non-daily smokers, nor an interaction between smoking group and SOA condition.

Exploratory analyses were conducted to investigate whether this could be due to the way the smokers were categorised as daily and non-daily smokers. Previous research in attentional bias used different criteria for their smoking groups such as the number of cigarettes per day (Hogarth et al. 2003) and their FTCD score (VollstädtKlein et al. 2011). Smokers were reclassified using the number of cigarettes per day 
and FTCD score. Both of these reclassifications did not change the outcomes with no significant difference between smoking groups, nor an interaction between smoking group and SOA condition. Further exploratory analyses were conducted to investigate whether the pattern of results supported a null effect. This was supported by a Bayesian equivalent of the $2 \times 2$ mixed ANOVA which showed that there was moderate evidence for a null effect over smoking group, and strong evidence for a null effect over the interaction between smoking group and SOA condition. Overall, this suggests there was not a meaningful difference in attentional bias between daily and non-daily smokers. As the effect sizes of Studies One and Two were in opposite directions, the results were combined using a mini meta-analysis to synthesise the effect sizes across studies.

\subsubsection{Meta-analysis}

Mini meta-analyses can be conducted on a small number of studies in order to synthesise potentially counterintuitive findings and increase statistical power through combining the participants of several studies (Goh, Hall, and Rosenthal 2016). Two separate meta-analyses were performed, one for each of the SOA conditions included in both studies (200ms and 500ms). When conducting meta-analyses, there is a choice between using a fixed effects model and a random effects model (Borenstein et al. 2010). A fixed effects model assumes each study is measuring the same effect size but differs due to sampling error. A random effects model assumes there is not one true effect size, and each study exists along a distribution of effects. For this analysis, a random effects model was used as Studies One and Two have several differences. The sample size of Study Two was much larger, the participants were older, different criteria were used to categorise the smokers, and participants completed the experiment online instead of in the lab. Therefore, the effects from each study were not assumed to be identical, and a random effects model was used. The results were converted to Cohen's d in order to express a standardised mean difference in attentional bias index between smoking groups.

Across both studies, the effect for a 200ms SOA was not significant, $d=0.03,95 \%$ $\mathrm{CI}=[-0.37,0.43], \mathrm{Z}=0.14, p=.890$. Similarly, the effect for a 500ms SOA was not significant, $\mathrm{d}=0.09,95 \% \mathrm{CI}=[-0.44,0.63], \mathrm{Z}=0.35, p=.730$. The results are shown as 
a forest plot for each SOA condition in Figure 3.10. This shows that synthesising the results of both studies returns a very small effect size. However, even with a combined sample of 206 participants, there were large confidence intervals that spanned from a small to moderate negative effect in favour of daily smokers displaying greater attentional bias, to a small to moderate positive effect size in favour of non-daily smokers displaying greater attentional bias.

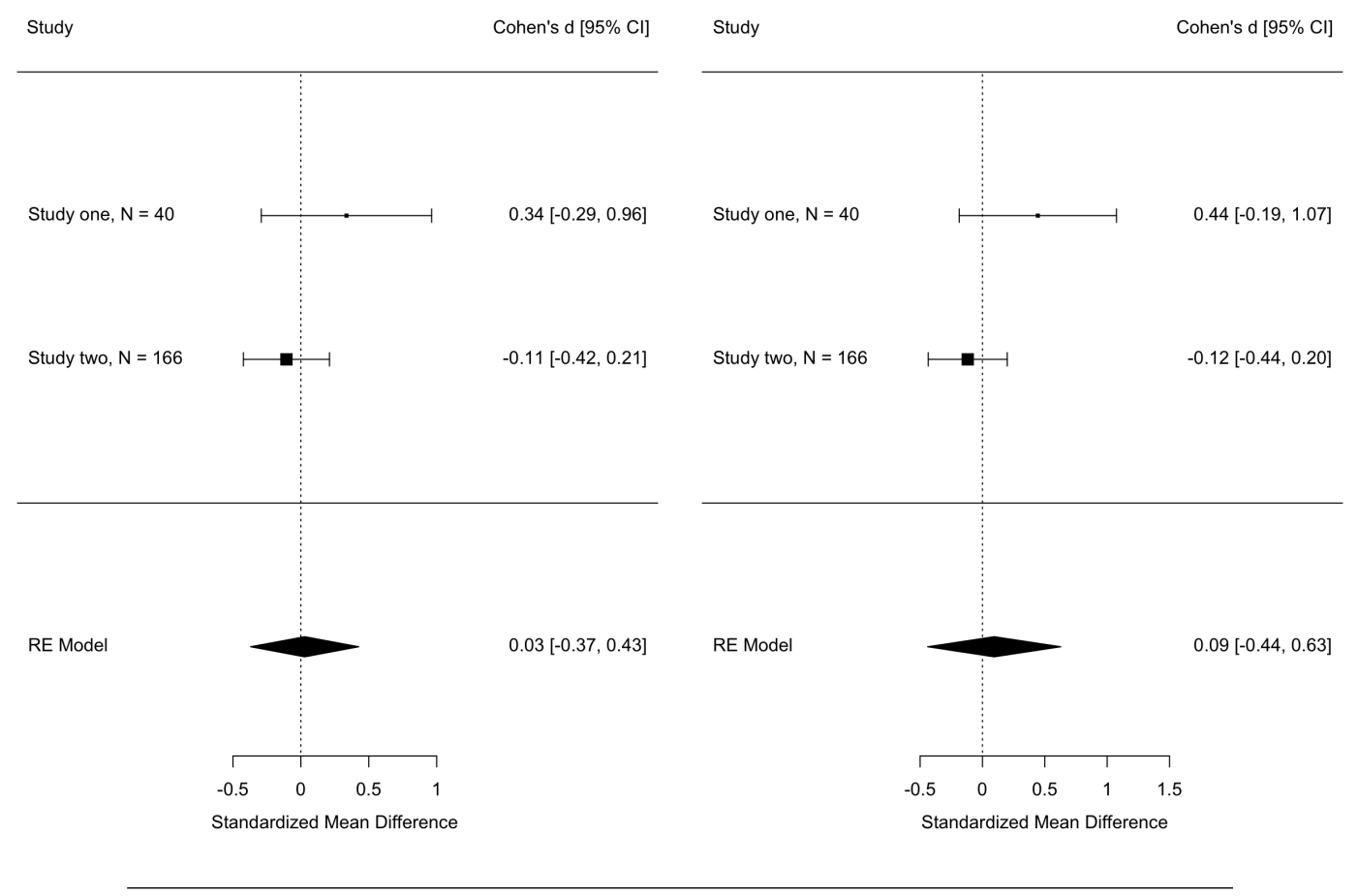

FIGURE 3.10: Forest plots show the meta-analytic effect size for Studies One and Two. The left plot shows Cohen's $\mathrm{d}=0.03,95 \% \mathrm{CI}=[-0.37$, 0.43 ] for the $200 \mathrm{~ms}$ SOA condition. The right plot shows Cohen's $\mathrm{d}=$ $0.09,95 \% \mathrm{CI}=[-0.44,0.63]$ for the $500 \mathrm{~ms}$ SOA condition .

\subsection{General discussion}

Previous research investigating attentional bias in daily and non-daily smokers reported conflicting results. Some studies found that non-daily smokers exhibited greater attentional bias towards smoking cues than daily smokers (Bradley et al. 2003; Hogarth et al. 2003; Mogg et al. 2005). On the other hand, other studies found that 
daily smokers displayed greater attentional bias towards smoking cues than non-daily smokers (Zack et al. 2001; Chanon et al. 2010; Vollstädt-Klein et al. 2011). Across the two studies presented in this chapter, there was not a meaningful difference between the smoking groups. Study One produced inconclusive results due to the relatively small sample size. Therefore, the lack of effect could have been reasonably due to a lack of statistical power rather than evidence for no effect. Study Two addressed these limitations and focused on increasing statistical power and consolidating the design of the study. With a much larger sample size, there were still no significant effects, and Bayesian exploratory analyses showed that this provided moderate to strong evidence in favour of a null effect. In further exploratory analyses, there were negligible effect sizes between daily and non-daily smokers when alternative attentional bias indexes (orienting and disengaging) were used. Finally, the results of Studies One and Two were synthesised in a mini meta-analysis in order to calculate the average effect size across both studies. Despite a combined sample size of 206 participants, there was no significant effect across both SOA conditions and the meta-analytic effect sizes were very small with large confidence intervals. Therefore, the studies in this chapter suggest there is not a meaningful difference in attentional bias towards smoking cues between daily and non-daily smokers. There are two ways to interpret these results. First, these two studies accurately represent no meaningful difference in attentional bias between the smoking groups. Second, there are potential reasons why there could be a meaningful difference in attentional bias, but these studies were unable to detect it. These possibilities are explored in turn.

Studies One and Two might represent no meaningful difference in attentional bias as previous published research may have problems with inflated effect sizes due to low statistical power. The largest known sample size in previous research was 51 smokers used by Vollstädt-Klein et al. (2011). In the simplest scenario, splitting these into two groups of 25 and 26 participants, a sensitivity power analysis using G*Power (Faul et al. 2009) indicates that this sample size would be sensitive to detect effect sizes of Cohen's $\mathrm{d}=0.80$ (alpha $=.05$, power $=.80$ ). Incidentally, a recent study showed that the median effect size in a random selection of 684 articles that were not pre-registered was Cohen's d $=0.80$ (Schäfer and Schwarz 2019). In the long-run, G*Power shows that Study Two would have 99\% power to detect an effect size of 0.80 . 
Therefore, it is unlikely the effect between daily and non-daily smokers is this large, or Study Two would have had enough power to detect it. In contrast, 89 articles that were pre-registered had a median effect size of Cohen's d = 0.35 (Schäfer and Schwarz 2019). This is due to pre-registered articles having larger sample sizes. Larger sample sizes reduce the sampling error, and typically produce smaller effect sizes (Open Science Collaboration 2015). The problem here is that small sample sizes are only sensitive enough to detect large effects, and due to publication bias where only significant results are published, studies with smaller effects are not reported (Etz and Vandekerkhove 2016). Study Two had the largest known sample size to investigate attentional bias with 60 non-daily smokers and 106 daily smokers. A sensitivity power analysis shows that this was sensitive to detect effect sizes of Cohen's $d=0.46$ (alpha $=.05$, power $=.80)$. Although it was not powered to detect an effect as small as $\mathrm{d}$ $=0.35$, Study Two was sensitive to detect an effect size of almost half the size of the next biggest sample in Vollstädt-Klein et al. (2011). This suggests that previous studies in attentional bias represent gross overestimates of any effect due to overinflated effects from small sample sizes. No previous study reported a power analysis which makes it difficult to ascertain what effect sizes they were interested in. Given that exploratory Bayesian analyses suggested there is moderate to strong evidence in favour of a null model in Study Two, there may not be a meaningful difference in attentional bias between smoking groups, at least in its current implementation where the effect is assumed to represent stable trait-like group differences.

Overinflated effect sizes from small samples may be combined with an issue in how attentional bias is typically studied. Attentional bias may not be a trait-like phenomenon that can produce stable differences between groups. Field, Werthmann and Franken (2016) outlined a contemporary theory of attentional bias and suggested that it could vary from moment to moment depending on how substance cues are being evaluated. This suggests that rather than being a stable trait between smoking groups, it fluctuates with the incentive value of a cue which makes within-group differences more important. Initial support for this theory was provided by Begh, Smith and Ferguson (2016) who found that laboratory measures of attentional bias such as the Stroop task and the visual probe task did not predict subsequent smoking behaviour in the real-world. However, assessments of craving and awareness of smoking cues 
in the environment measured through ecological momentary assessment did predict subsequent smoking behaviour. Therefore, the conflicting results in previous studies and the null results in these two studies may be a product of the fluctuating nature of attentional bias in response to momentary evaluations of smoking cues (Field et al. 2016). In smaller samples, attentional bias could fluctuate one way or the other, but in larger samples like Study Two, the fluctuating nature of attentional bias would cancel out and converge to a mean difference around zero. Therefore, future research may benefit from investigating which factors affect the momentary evaluation of substance cues and the subsequent expression of attentional bias.

It may be important to explore what factors lead to changes in the momentary evaluation of smoking cues in different smoking groups as it is well established that lighter and heavier smokers report different smoking motives. Daily smokers are predominantly motivated by the physical indicators of nicotine dependence such as craving and habit (Shiffman et al. 2012a; Scheuermann et al. 2015). On the other hand, nondaily smokers are characterised by being responsive to smoking cues and smoking in specific situations (Darlow and Lobel 2012; Shiffman et al. 2012a; Shifmann et al. 2014b). As daily and non-daily smokers have different smoking motives, they may not evaluate substance cues in the same way. Therefore, it would be interesting to investigate how attentional bias fluctuates when the momentary evaluation of substance cues changes. One influence could be how smoking cues are evaluated in the presence of alcohol. There appears to be a special relationship between smoking and drinking alcohol, and this relationship appears to be particularly pronounced in nondaily smokers (Tong et al. 2006; Hoeppner et al. 2014). Non-daily smokers are known to increase their smoking intake when drinking alcohol (Witkiewitz, Desai and Steckler 2012), and experimental research has shown that cigarette craving increases after consuming alcohol (McGrath, Peloquin and Ferdinand 2015). Therefore, exploring how attentional bias towards smoking cues is affected by different influences could be the next step in research. This would help determine whether there is no meaningful difference in attentional bias between daily and non-daily smokers, or whether investigating attentional bias as a stable trait-like characteristic has limited what can be learnt from only exploring between group differences.

The visual probe task is popular, but it may be a problematic measure of attentional 
bias. It is no secret that there are vocal critics of the task due to its questionable level of internal consistency (Schmukle 2005; Ataya et al. 2012). Previous estimates have ranged between 0 (Waechter et al. 2014) and .28 (Schmukle 2005). A reliability estimate of 0 means there is no reliability as each trial in the task appears to be measuring a different construct (Henson 2001). Study One aimed to improve the psychometric properties of the task by using individualised smoking stimuli. This was based on Christiansen et al. (2015) as they demonstrated that including individualised alcohol stimuli led to an adequate level of internal consistency. However, in Study One, providing smoking participants with individualised smoking cues still produced a task with poor levels of internal consistency. Study Two also had suboptimal levels of internal consistency and split-half reliability. The previous improvement by Christiansen et al. (2015) failed to replicate in a range of studies (Jones et al. 2018). Low internal consistency suggests the visual probe task is not reliably measuring a single process (Clayson and Miller 2017). It also has implications for statistical power as lower internal consistency caps statistical power (Henson 2001). Kruijt, Field and Fox (2016) showed in simulations that attentional bias cannot be isolated from the high measurement error of the visual probe task. The reliability of cognitive tasks is rarely reported unless it is the main focus of the article (Parsons et al. 2019), which means it is difficult to fully assess how reliable the tasks were in previous research. Low reliability may not be as problematic for experimental research as unreliable tasks can produce consistent effects between groups (Hedge et al. 2018), but in order to investigate momentary evaluations of smoking cues on attentional bias, several measurements would be needed. This would require a stable internally consistent measure, which the visual probe task has been consistently shown not to be. For future research, it may be beneficial to use eye-tracking as a direct measure of visual attention to measure attentional bias. This has several desirable properties such as larger effect sizes (Field et al. 2009), higher internal consistency (Waechter et al. 2014; Christiansen et al. 2015; Price et al. 2015), and the fact that it does not rely on inferring the location of attention, but directly measuring it. If future research was to focus on measuring how attentional bias fluctuates instead of being a stable trait-like characteristic, then using response times from visual probe task in isolation is strongly discouraged.

Alternative explanations for the lack of effect could mean there might be an effect 
like in previous studies, but the design of Studies One and Two masked it. First, there was evidence for the null in favour of effects of smoking group or an interaction in Study Two. However, there was a key methodological difference in Study Two with the experiment being conducted online as opposed to being in a controlled lab situation. This meant the participants completed the experiment on their own computers in an environment of their choosing. This creates more heterogeneity, but studies have shown that online experiments utilising cognitive tasks replicate as well as if they were performed in the lab (Germine et al. 2012; Crump et al. 2013; Hilbig 2016). The only limit was subliminal presentation of stimuli (64ms or less), which was not the case in this experiment as the fastest presentation time was $200 \mathrm{~ms}$. Online studies produce greater variance in responses (Brand and Bradley 2012; Hilbig 2016), but with a sample size three times as large as the next biggest study, this was more than compensated for. Furthermore, following the recommendations of Clifford and Jerit (2014), participants were removed if they experienced any technical issues which could affect measurements. Although the experiment being performed online was a key methodological difference, it followed stringent recommendations from studies replicating cognitive tasks online. Therefore, it is unlikely to explain the lack of effect here.

Second, the sample in Studies One and Two consisted of predominantly white caucasian participants, with 85\% of participants in Study One, and 93\% in Study Two. This may be problematic for studying differences between daily and non-daily smokers, as there is consistent evidence that non-daily smoking is more prevalent in ethnic minority groups (Fagan and Rigotti 2009; Levy et al. 2009). In addition, this issue was also reflected in the images used in the visual probe task as the models were a white male and female. However, this is unlikely to have contributed to the lack of effect in attentional bias. Birkás, Dzhelyova, and Lábadi (2014) showed that faces are evaluated differently depending on ethnicity. There is an own ethnicity bias in caucasians, as they evaluate faces as being more trustworthy even when features are manipulated to be less trustworthy. This suggests that as the sample was predominantly white and the images contained white models, it is unlikely the smaller proportion of nonwhite participants had a large enough counteractive effect to diminish any effect on attentional bias. Therefore, the main limitation of the sample in this study is a lack of generalisability to other ethnicities, especially those where non-daily smoking is more 
prevalent.

Taking these considerations into account, the most plausible explanation is the results could represent a null effect due to previous studies reporting a combination of low statistical power and the fluctuating nature of attentional bias. If attentional bias fluctuates based on the evaluation of smoking cues, it may not be best studied as a stable trait between groups. Small sample sizes can produce inflated effect sizes due to larger sampling error (Open Science Collaboration 2015). Inflated effect sizes are worsened by publication bias, as only significant results are published, meaning studies with smaller, more realistic effects are not reported (Etz and Vandekerkhove 2016). This means in smaller studies, attentional bias can fluctuate one way or the other in favour of lighter or heavier smokers. These are then published as significant but inflated effects. When a larger study was performed in the case of Study Two, these fluctuations cancelled out as sampling error decreased, and there was not an observable trait like difference between groups in attentional bias.

\subsubsection{Strengths and limitations}

There are some strengths and limitations that should be acknowledged about the studies presented in this chapter. The strengths include the large sample size of Study Two. Recruiting a larger sample size addressed the limitation of the sample size in Study One being determined by previous research. Using a simulated power analysis, the sample size was set to be powered for the smallest effect size of interest, which resulted in a sample size three times larger than the next biggest study on attentional bias in daily and non-daily smokers. This helped to provide enough evidence in favour of a null effect using Bayesian statistics, rather than an inconclusive non-significant effect. In addition, conducting the experiment online helped to recruit a more diverse sample in terms of age, country of origin, and education history. This also avoided relying on university undergraduates as the primary sample.

There were also some limitations to consider when interpreting the results. First, the sample in Study Two may have been more diverse in some ways, but both studies still contained predominantly white participants. Non-daily smoking is more prevalent in ethnic minority groups (Fagan and Rigotti 2009; Levy et al. 2009), and the health implications of smoking disproportionately affect non-white smokers (St.Helen et al. 
2019). Therefore, future research would benefit from recruiting a larger proportion of non-white smokers, in order for the results to generalise beyond white smokers.

Second, in Study One participants completed one of two versions of the visual probe task containing either roll-up cigarette or manufactured cigarette stimuli depending on their self-reported preferences. The aim of this was to explore whether using individualised stimuli improved internal consistency. Although there was not a higher level of internal consistency, participants only completed one version of the task. In contrast, Christiansen et al. (2015) compared the internal consistency of the visual probe task for both general and individualised stimuli in the same participants. This enabled them to investigate whether it was the stimuli that led to the increase in internal consistency. As participants only completed one task version in Study One, there was no way to determine how the individualised stimuli would have compared to a set of generic stimuli.

Third, the online nature of Study Two meant the participants' smoking levels could not be verified objectively using $\mathrm{CO}$. Measuring exhaled $\mathrm{CO}$ in one session has been shown to be an effective measure of cigarette use (Wray et al. 2015). Nevertheless, Ramo et al. (2011) demonstrated that smoking-related information collected online has good reliability and validity. This means the lack of $\mathrm{CO}$ verification should be acknowledged as a limitation, but the smoking measures were not invalid.

Fourth, as participants completed the study in an environment of their choosing, there was no control over the time since their last cigarette. Daily and non-daily smokers have naturally different smoking rates which was demonstrated perfectly in Study Two: daily smokers had a cigarette an hour prior to the study on average, while nondaily smokers last smoked two days prior on average. This did lead to some idiosyncrasies as some smokers reported to smoke while they were completing the study. In Study One, all smokers had their last cigarette at least one hour prior to the beginning of the study by the time participants began the experiment. This may be a limitation as the longer the time since the last cigarette, the greater the craving which can increase attentional bias towards smoking cues (Field and Cox 2008; Field et al. 2016). Differences in the time since their last cigarette may represent a more naturalistic environment for the smokers as non-daily smokers typically do not increase craving within two days of their last cigarette (Shiffman et al. 2012b). Nevertheless, this ultimately 
meant Study Two had less control over the smokers' deprivation levels.

Fifth, a coding error prevented the design of the the visual probe task in Study Two being closer to the task used in Study One. The 200ms SOA condition for neutral control trials was duplicated instead of creating trials for the 500ms SOA condition. Salemink et al. (2007) highlighted that traditional versions of the visual probe task do not distinguish whether attentional bias is the product of vigilance towards smoking cues or a difficulty with disengagement causing slower responses on nonsmoking trials. By comparing the response times to a set of neutral control trials, the distinction can be made between vigilance and disengagement by comparing whether attentional bias is caused by faster responses to smoking cues, or slower responses to non-smoking cues. Due to the coding error, only the $200 \mathrm{~ms}$ SOA condition could be explored, but consistent with the confirmatory analyses, there was still no significant difference between daily and non-daily smokers. It is unlikely the missing 500ms SOA condition would have produced alternate results as all the attentional bias indexes in the 200ms condition had negligible effect sizes.

Finally, a random effects model was used for the mini meta-analysis. This model was chosen due to the heterogeneity in methods between Studies One and Two, and a fixed effect size was not assumed. However, a limitation of a random effects model is when a small number of studies are used, it produces an imprecise estimate of the between-study variance (Borenstein et al. 2010). This makes it more difficult to interpret the results due to the lack of information on the between-study variance. Considering a fixed effects model would have been inappropriate, a mixed effects model was used while acknowledging the limitations. Furthermore, internal mini meta-analyses are descriptive in nature and can increase the type I error rate if some studies are suppressed (Vosgerau et al. 2018). The reader will need to take it on face value that this analysis included all the studies on attentional bias conducted for this thesis, but the effect was very small as opposed to being artificially inflated. 


\subsubsection{Conclusion}

The aim of this chapter was to investigate the mixed results in previous attentional bias research highlighted in the systematic review presented in Chapter Two. Some studies reported that non-daily smokers showed greater attentional bias towards smoking cues, while others reported that daily smokers showed greater attentional bias. The two studies presented in this chapter attempted to clarify this pattern of results. Both studies produced null results, with Study Two providing moderate to strong evidence in favour of the null model over models including the smoking group and an interaction between smoking group and the SOA condition. When the effect sizes of Studies One and Two were combined in a mini meta-analysis, the combined effect sizes were very small with wide confidence intervals. The most plausible explanation for the null results is a combination of two factors in typical attentional bias experiments. Attentional bias may not be a stable trait between groups, but fluctuates with the evaluation of smoking cues. Most attentional bias experiments, including these two studies, measure attentional bias once without manipulating factors that can affect the evaluation of smoking cues. Therefore, each study captures a limited view of attentional bias fluctuating in daily and non-daily smokers. The fluctuating nature of attentional bias is combined with previous studies being underpowered and reporting potentially inflated effect sizes when attentional bias coincidentally peaked in daily or non-daily smokers. When a larger sample size is used, as in Study Two, these fluctuations and peaks are cancelled out, and there is no meaningful difference between daily and non-daily smokers when attentional bias is measured as a stable between-group trait. Future research would need to explore whether manipulating how smoking cues are evaluated would affect the expression of attentional bias.

Chapter Three presented research focusing on the drive component of the iRISA model of addictive behaviour. In the next chapter, the focus turns to cognitive control as the second component of the iRISA model. Where drive processes like attentional bias are predicted to stimulate drug use, control processes are theorised to contribute to the ability to inhibit or adapt behaviour. In the systematic review presented in Chapter Two, there were disparate studies investigating inhibitory control and error processing. Across three studies, inhibitory control was explored using three different 
paradigms, and the one study focusing on error processing produced counter-intuitive results. Therefore, Chapter Four presents one study exploring inhibitory control and error processing in daily and non-daily smokers to target the gap identified in the systematic review. 


\section{Chapter 4}

\section{Error Processing and Inhibitory}

\section{Control}

\subsection{Introduction}

The second area of interest highlighted in Chapter Two was cognitive control as it received marginal attention in previous research when considering daily and non-daily smokers. Cognitive control is an umbrella term for different processes that regulate behaviour, including inhibitory control and error processing. Three studies explored inhibitory control and one study explored error processing in lighter and heavier smokers. Given the idiosyncratic methods used in these studies, Chapter Four focuses on further investigating cognitive control in daily and non-daily smokers.

Cognitive control is the second key component of the iRISA model of drug addiction (Goldstein and Volkow 2011; Zilverstand et al. 2018). Where attentional bias may contribute to the drive to use a particular drug, cognitive control is the ability to control or adapt behaviour. Inhibitory control and error processing are two key facets of cognitive control which share a neural network (de Ruiter et al. 2012; Luijten et al. 2014). Inhibitory control stops or inhibits inappropriate behaviour, and error processing (alternatively known as performance monitoring) identifies mistakes for adapting future behaviour. Both of these processes are complementary in the sense that they provide the ability to stop a behaviour and then learn from these for future situations which is key for adaptive behaviour. 
Inhibitory control is the ability to withhold a behavioural response once it has begun to be initiated (Smith et al. 2014), and successfully withholding an inappropriate response allows you to choose a more appropriate action (Verbruggen and Logan 2009). Deficits in inhibitory control may be a proxy for difficulties in controlling drug use, as if smoking becomes an automatic process, a deficit in inhibitory control means it would be more difficult to resist smoking once the initial behaviour has been initiated (Luijten et al. 2011a). For example, if someone was trying to quit smoking and they reach for a cigarette out of habit, it may be difficult to suppress the response once it has been initiated.

Error processing is the ability to learn from mistakes and is part of the general ability of performance monitoring (Luijten et al. 2011b; Rass et al. 2014). Learning from previous experience is key to adapting, but drug dependent users are characterised by their inability to adapt their behaviour based on previous negative experiences (Luijten et al. 2014). Like inhibitory control, tasks measuring error processing are intended to be a proxy for problematic drug use. Deficits in error processing would be failing to adapt behaviour despite adverse consequences, either in personal health or family life (Franken and van der Wetering 2015).

\subsubsection{The association between cognitive control and risk of relapse}

These two facets of cognitive control are important as they may be associated with the risk of relapse. Three months after attempting to give up smoking, an electrophysiological measure of inhibitory control - the P3 ERP component - was negatively associated with the risk of relapse (Luijten et al. 2016). Lower inhibitory control (indicated by lower P3 amplitude) was associated with an increased risk of relapse. This may also be the case for fMRI measures of inhibitory control. Before monitoring craving and subsequent smoking in participants for 21 days after cessation, participants completed an inhibitory control task (Berkman et al. 2011). Greater activation in regions associated with inhibitory control such as the inferior frontal gyrus and basal ganglia were associated with a weaker relationship between craving and smoking. This shows that greater inhibitory control can moderate the relationship between wanting to smoke a cigarette and being able to withhold the behaviour. This also appears to be the case for young, relatively inexperienced smokers. Before enrolling on a four-week 
high-school smoking cessation programme, a group of 14-18 year olds completed a battery of measures including a continuous performance task where participants had to withhold a response to particular stimuli (Krishnan-Sarin et al. 2007). Although the sample size was very small, the 14 participants who relapsed made more errors where they failed to avoid responding to the stimuli. This shows that the participants who relapsed found it harder to inhibit prepotent responses, demonstrating poorer inhibitory control. Measuring impulsivity using a questionnaire is also associated with relapse. Before a group of low socioeconomic status smokers completed cognitive behaviour therapy, they completed a battery of measures including behavioural inhibitory control (Go/NoGo task) and cognitive impulsivity using the Behavioural Inhibition Scale (Sheffer et al. 2012). Although the results of the Go/NoGo task were not reported, greater cognitive impulsivity was associated with higher relapse rates. This collection of studies shows participants who display poorer inhibitory control across behavioural and neuroimaging methods have worse treatment outcomes, either relapsing or finding it harder to resist smoking. Given daily and non-daily smokers have similar relapse rates when attempting to quit smoking (Tindle and Shiffman 2011; Kotz et al. 2012; Herbec et al. 2014), understanding whether they show similar deficits in cognitive control would be an important development for identifying those at risk of relapse during a quit attempt, or monitoring the effectiveness of an intervention.

\subsubsection{Inhibitory control and smoking}

Starting with inhibitory control, there is evidence across different methods in favour of smokers showing greater deficits in comparison to non-smokers. The Go/NoGo task is frequently used where participants must respond rapidly to a frequent stimulus (Go), but inhibit their response to an infrequent stimulus (NoGo). The infrequency of the stimulus creates a prepotent response which requires participants to inhibit their behaviour (Wessel 2018). When the stimuli are presented rapidly, smokers made significantly more errors on both Go (omission errors) and NoGo (commission errors) trials in comparison to non-smokers (Zhao et al. 2016). This suggests that using behavioural measures, smokers have worse inhibitory control where they provide an inappropriate response regardless of the trial. 
In ERP measures of inhibitory control, studies have focused on the N2 and P3 components which have greater activity during NoGo trials than Go trials. Different studies have shown smokers have lower amplitude than non-smokers in one component or the other, but rarely both in the same study. Smokers had lower N2 amplitude on NoGo trials in comparison to non-smokers (Luijten et al. 2011a; Buzzell et al. 2014). Using an oddball task, a large sample of smokers had a significantly lower P3 amplitude than non-smokers and ex-smokers (Anokhin et al. 2000). Using the more standard Go/NoGo task, Evans et al. (2009) found that both satiated and abstinent smokers had lower P3 amplitude than non-smokers. There is consistently a deficit in ERP measures of inhibitory control in smokers in comparison to non-smokers, but there is less consistency with the specific component.

With its greater spatial resolution, fMRI measures focus on activity in different regions of the brain (Woodman 2010). During inhibition, studies showed that smokers had lower activity than non-smokers in regions associated with inhibitory control such as the anterior cingulate cortex, dorsomedial prefrontal cortex, and inferior frontal gyrus (de Ruiter et al. 2011; Nestor et al. 2011; Luijten et al. 2013). There was not a significant difference in activity during inhibition between smokers and non-smokers in Galvan et al. (2011), although there was a negative relationship between heaviness of smoking and activity during inhibition in smokers. This shows that there may not have been a significant difference between smokers and non-smokers, but heavier smokers showed greater deficits than relatively lighter smokers. As a whole, this body of literature found that smokers, in comparison to non-smokers, show deficits in inhibitory control as indexed by lower ERP amplitudes or lower Blood-Oxygen-LevelDependent (BOLD) activity.

\subsubsection{Error processing and smoking}

There is a similar pattern of results in error processing, where daily smokers show deficits in recognising they have made mistakes in comparison to non-smokers using behavioural and neuroimaging methods. Participants completed a reinforcement learning task where they had to learn rules after receiving rewards or punishments on each trial (Butler et al. 2017). Smokers took longer to recognise a change in rules than non-smokers and former smokers, and did not reduce their response time as much 
after making a mistake. This shows that using a behavioural measure, smokers found it more difficult to adapt their behaviour after making mistakes.

In ERP measures of error processing, participants usually complete a speeded response time task such as the Eriksen Flanker. Correct trials are compared to incorrect trials to investigate whether participants recognise making mistakes. Studies have usually focused on the Error Related Negativity (ERN) and Positive error (Pe) components which typically have greater ampltitude after participants make a mistake. Smokers have consistently lower Pe amplitude than non-smokers during incorrect trials (Franken et al. 2010; Luijten et al. 2011b), but only one study observed significantly lower ERN amplitudes in smokers (Luijten et al. 2011b). Similar to inhibitory control, this research suggests smokers display deficits in ERP components of error processing, but it is not clear whether this is consistent across both components.

Studies using fMRI measures use similar speeded response-time tasks, where they compare BOLD activity on correct and incorrect trials in regions of interest. Smokers typically have lower activity during incorrect trials in comparison to non-smokers, predominantly in the right middle and right superior frontal gyrus (Nestor et al. 2011) and dorsal anterior cingulate cortex (de Ruiter et al. 2011). Conversely, Carroll et al. (2015) found there was not a significant difference between smokers and non-smokers, but there was a negative relationship between BOLD activity and externalising personality traits in smokers. This suggests that a higher externalising personality trait which encapsulates impulsive behaviours - is associated with lower BOLD activity in response to errors in the dorsal anterior cingulate cortex and right insular. However, given the low sample size and supplementary analyses, this result should be interpreted tentatively. Together, these studies show that smokers typically display lower BOLD activity in regions associated with error processing than non-smokers.

There is converging evidence that smokers, in comparison to non-smokers, display deficits in inhibitory control and error processing through ERP and fMRI measures. However, all of the research stipulated only daily smokers could participate, with the most inclusive study using five or more cigarettes per day. This means the vast majority of research in the area has excluded non-daily smokers. 


\subsubsection{The meaning of cognitive control deficits}

In the previous sections, deficits in inhibitory control and error processing were established by comparing smokers and non-smokers on ERP amplitude in EEG studies and BOLD activity in fMRI studies. Deficits were defined as performance relative to nonsmokers, but this does not necessarily mean smaller neurocognitive responses lead to worse behavioural outcomes. Behavioural indices of inhibitory control and error processing were rarely significantly different between smokers and non-smokers. These observations could be interpreted in two ways. The first is that there may be differences in neurocognitive measures, but it may not be reflected in actual behaviour. The second is behavioural effects are smaller than neuroimaging measures, but the sample sizes typically used in this area of research are not big enough to reliably detect them. This option could also be interpreted as neurocognitive measures are a more sensitive marker of deficits in cognitive control performance. However, supporting one of these options is difficult as previous studies have not focused on effect sizes or establishing normative levels in order to rank performance. For example, Brinkmeyer et al. (2011) established deficits in smokers by comparing them to non-smokers. Deficits were indicated by smaller P50 amplitude relative to non-smokers, but in the supplementary materials, the authors provide normative levels of performance for smokers and non-smokers. The next step would be to link these neurocognitive deficits to specific behavioural outcomes. This means we typically do not know whether deficits relative to non-smokers represent real behavioural problems that affect smoking. Therefore, it is important to keep in mind both the relative performance of neurocognitive mechanisms in smokers compared to non-smokers, and whether these deficits impact behaviour.

\subsubsection{Cognitive control in daily and non-daily smokers}

The studies reported in the systematic review in Chapter Two provided an idiosyncratic exploration of inhibitory control in different smoking groups. Using behavioural measures, heavier smokers had lower performance than lighter smokers (Billieux et al. 2010) and non-smokers (Yakir et al. 2007). However, Yakir et al. (2007) did 
not report the comparison between current and occasional smokers. Heavy smokers showed deficits in P50 gating, a form of inhibitory control of repetitive auditory tones, in comparison to light smokers and non-smokers (Brinkmeyer et al. 2011). Light smokers and non-smokers were not significantly different. This shows that using different measures, heavier smokers consistently perform worse on inhibitory control than non-smokers. Lighter smokers normally hold an intermediary position, but not significantly different to non-smokers.

There only appears to be one study that has purposely included non-daily smokers when investigating error processing. Rass et al. (2014) explored error processing in intermittent and daily smokers. Intermittent smokers were defined as smoking on fewer than 27 days per month, which means the sample was more inclusive than other ERP research in cognitive control. Rass et al. (2014) found that intermittent smokers had greater Pe amplitude on incorrect trials than daily smokers using a Go/Nogo task. Intermittent smokers had greater amplitude than non-smokers, but this difference was not statistically significant. Their interpretation was intermittent smokers may possess a kind of protective characteristic, demonstrated through higher Pe amplitude, which allows them to smoke infrequently and prevent them from transitioning into nicotine dependence.

The finding that non-daily smokers may outperform daily smokers is largely consistent with other research included in the systematic review in Chapter Two. Studies that have included lighter and heavier smokers have either found similar neurocognitive deficits, or worse deficits in heavier smokers. However, outperforming nonsmokers, albeit not a significant difference, has not been reported in previous studies. This opened up two avenues for the study presented in this chapter. First, the findings from Rass et al. (2014) are largely inconsistent with previous research. As the only study reporting non-daily smokers may outperform non-smokers, it is important to replicate the study to see if a similar pattern of results can be observed. Second, Rass et al. (2014) report that their findings relate to both error processing and inhibitory control, but this is not consistent with their methods. Their study used two cognitive tasks: the Eriksen Flanker and Go/NoGo task. While the Go/NoGo task is commonly used to study inhibitory control, this study compared correct and incorrect trials and measured the ERN and Pe ERP components. These are used as 
indices of error processing. Only Brinkmeyer et al. (2011) explored ERP measures of inhibitory control, but this was not based on a speeded response-time task. Therefore, the study presented in this chapter aimed to contribute to the field of addictive behaviour in two key ways. The first aim was to replicate the comparison between daily and non-daily smokers on error processing. The second aim was to extend the area of research by comparing daily and non-daily smokers on inhibitory control using a speeded response-time task.

The tasks included in this study focused on ERP measures of cognitive control. Few studies reported behavioural effects between groups, potentially as they would require more participants than are typically used in neuroimaging studies to provide an appropriate level of statistical power. Conti et al. (2019) reported that there was a small non-significant effect in a meta-analysis of four studies focusing on motor impulsivity. Alternatively, fMRI measures could be used but there are two main problems. Logistically, there were no facilities in this institution to conduct fMRI experiments. Practically, fMRI experiments are expensive which creates barriers for experiments and future applied use. The end goal of investigating cognitive control deficits in daily and non-daily smokers is applying the insights to improve diagnosis and cessation outcomes. EEG equipment is relatively inexpensive and mobile in comparison to MRI scanners. If ERPs can provide reliable biomarkers for smokers at risk of relapse or evaluate the effectiveness of cessation interventions, they would provide a more practical solution to a larger number of patients (Houston and Schlienz 2018). This means that ERP experiments have the capability to provide insights into the temporal properties of neurocognitive deficits with the practical value of being available in an applied treatment setting.

\subsubsection{ERP components associated with inhibitory control}

The Go/NoGo task presents participants with a frequent Go stimulus that requires a rapid response, but on a small number of trials a NoGo stimulus is presented which requires the response to be suppressed. There are two ERP components observed during NoGo trials that require inhibiting a response: the N2 and the P3. The N2 is a negative wave appearing between $200-300 \mathrm{~ms}$ post-stimulus presentation on trials that 
require the suppression of a pre-potent response (Luijten et al. 2014). It has a frontocentral distribution with neural generators appearing to include the inferior frontal cortex, mid-cingulate cortex, and anterior cingulate cortex (Huster et al. 2013; Luijten et al. 2014). The N2 may reflect the conflict monitoring and decision making process preceding a suppressed response (Kok, Ramautar, and de Ruiter 2004).

After the N2, the $\mathrm{P} 3$ is a positive wave appearing between $300-500 \mathrm{~ms}$ post-stimulus presentation (Rietdijk, Franken and Thurik 2014). This component has more of a fronto-central to centro-parietal distribution with neural generators including the midcingulate cortex, precentral cortex, and motor and premotor cortices (Huster et al. 2013; Smith et al. 2013; Luijten et al. 2014). In contrast to the N2, the P3 may reflect a more direct process of the motor response being inhibited (Luijten et al. 2014). Together, these components reflect two processes underlying inhibitory control.

\subsubsection{ERP components associated with error processing}

The Eriksen Flanker task is typically used where participants must rapidly respond to congruent and incongruent stimuli. Encouraging the participant to respond rapidly leads to a number of errors. These error trials are then compared to trials where a correct response was provided. There are two ERP components present after making a mistake: the ERN and Pe. The ERN is typically present immediately after making an incorrect response, present as a negative wave $0-150 \mathrm{~ms}$ after an error (Rietdijk et al. 2014). The ERN has a fronto-central distribution (Wessel, Danielmeier, and Ullsperger 2011), with more consistent evidence suggesting that the neural generator of the ERN is the anterior cingulate cortex (Botvinick, Cohen, and Carter 2004; Luijten et al. 2014). The ERN is thought to represent the initial realisation of making an error (Luijten et al. 2011b).

This is followed by the Pe component which emerges 200-400ms after making a mistake (Rietdijk et al. 2014). This component has a wide spread distribution (Ridderinkhof, Ramautar, and Wijnen 2009), typically towards the parietal region, but the neural generator of the Pe is less certain (Wessel et al. 2011). The Pe component may represent the conscious evaluation of or motivational significance associated with making a mistake (Ridderinkhof et al. 2009; Luijten et al. 2014). The ERN and Pe are 
thought to reflect two independent components underlying error processing (Overbeek et al. 2005).

\subsubsection{Hypotheses}

The hypotheses for inhibitory control and error processing were pre-registered on the OSF (https://osf.io/frgsw/). Daily smokers were predicted to display a deficit in inhibitory control indicated by a smaller mean amplitude on NoGo trials in daily smokers than non-daily smokers and non-smokers. Non-daily smokers were expected to exhibit a smaller mean amplitude in response to NoGo stimuli than non-smokers. These effects were expected for both the N2 and P3 components.

A similar pattern of results was expected for error processing. Daily smokers were predicted to have a smaller mean amplitude on incorrect trials than non-daily smokers and non-smokers. In addition, non-daily smokers were expected to have a smaller mean amplitude on incorrect trials than non-smokers. The deficits were expected for both the ERN and Pe components.

\subsection{Methods}

\subsubsection{Participants}

All participants had to be between the ages of 18-50, fluent in speaking English, and have normal or corrected normal vision. In addition, non-smokers were excluded if they reported to have smoked more than 10 lifetime cigarettes. Smokers were required to have smoked for at least six months before participating in the study and to not have smoked in the hour preceding their study session to standardise the time before their last cigarette in all smokers. Daily smokers were required to smoke every day. Non-daily smokers were required to smoke on 4-27 days out of the month.

The required sample size was based on a reanalysis of Luijten et al. (2011b). Their original classification of smokers and non-smokers was changed to divide the smokers into a group of light and heavy smokers based on their FTCD scores as daily versus non-daily smoking information was not available. This resulted in a significant interaction between correctness and smoking group on ERN amplitude $(\mathrm{F}(2,24)=7.73, p$ $=.003, \eta G^{2}=.09$ ). $G^{*}$ Power (Faul et al. 2009) indicated that with an alpha level of .05, 
power of .80, 3 groups and 2 measurements, 105 participants were required to detect a within-between interaction with an effect size of $\eta G^{2}=.09$. As this was only one study utilising different methods, the aim was to use sequential analysis to improve the efficiency of data collection while still controlling the Type I error rate (Lakens 2014). The pre-registration protocol indicated that data collection should have paused when 18 participants were sampled in each group to conduct a preliminary analysis. As previous research did not consistently demonstrate an effect in all components for error processing and inhibitory control, data collection should have stopped if there was a significant interaction between smoking group and correctness/stimulus in at least one component. It was important that there should have been a significant effect for at least one component in both error processing and inhibitory control. If this was not the case, then data collection was planned to continue until the full sample of 35 participants per group were recruited. To control for an increase in Type I error rate due to optional stopping (Simmons et al. 2014), an adjusted alpha level of .029 was used based on the Pocock boundary outlined in Lakens (2014) for looking at the data twice. This was calculated using the gsDesign package (Anderson 2016) in R.

\subsubsection{Design}

For both tasks, the plan was to use a three-way $(3 \times 2 \times 3)$ mixed design. This consisted of one between-subjects IV of smoking group (non-smoker, non-daily smoker, or daily smoker), and two within-subject IVs of trial condition and electrode position (Fz, Cz, and $\mathrm{Pz}$ ). The trial condition in the Eriksen Flanker task was either a correct or incorrect response made by the participant, and the trial condition in the Go/NoGo task was either a Go or NoGo trial. For each task, there were two DVs based on ERP timewindows observed in previous research. These time-windows were pre-registered following the recommendations of Luck and Gaspelin (2017) to avoid finding spurious results resulting from post-hoc selection of ERP time windows. In the Eriksen Flanker task, the ERN was defined as the mean amplitude $(\mu V) 25-75 \mathrm{~ms}$ after making a response, and the Pe was defined as the mean amplitude $200-400 \mathrm{~ms}$ after making a response (Overbeek et al. 2005; Rietdijk et al. 2014; Luijten et al. 2016). In the Go/NoGo task, the N2 was defined as the mean amplitude $(\mu V)$ 175-250ms after stimulus onset, and the P3 was defined as the mean amplitude $300-500 \mathrm{~ms}$ after stimulus onset 
(Rietdijk et al. 2014; Luijten et al. 2016).

\subsubsection{Materials}

Participants completed the same questionnaires as Study One (see Section 3.3.1.3 for a description of each questionnaire and example items). The estimates for the internal consistency of each sub-scale of the WISDM-68 in this sample are reported in Table 4.1. The internal consistency (Cronbach's alpha) of the FTCD was marginally higher than in Study One but less than Study Two $(\alpha=.51,95 \% \mathrm{CI}=[.00, .78])$, but there are large confidence intervals due to the small number of smokers in the sample. There was a similar pattern of internal consistency estimates as Study One for the PANAS in the positive $(\alpha=.89,95 \% \mathrm{CI}=[.79, .93])$ and negative affect sub-scales $(\alpha=.79,95 \%$ $\mathrm{CI}=[.63, .87])$. The internal consistency of the QSU was similar to Study One with a higher estimate for factor $1(\alpha=.93,95 \% \mathrm{CI}=[.75, .97])$ than factor $2(\alpha=.81,95 \% \mathrm{CI}=$ $[.53, .95])$.

TABLE 4.1: Internal consistency estimates using Cronbach's alpha for each sub-scale of the WISDM-68.

\begin{tabular}{cccc}
\hline Sub-scale & Lower CI & Alpha & Upper CI \\
\hline Affiliative Attachment & .67 & .92 & .98 \\
\hline Automaticity & .74 & .89 & .96 \\
\hline Loss of Control & .02 & .85 & .96 \\
\hline Behavioural Choice & .30 & .77 & .90 \\
\hline Cognitive Enhancement & .94 & .98 & .99 \\
\hline Craving & .60 & .89 & .96 \\
\hline Cue Exposure & .24 & .75 & .91 \\
\hline Negative Reinforcement & .57 & .87 & .94 \\
\hline Positive Reinforcement & .40 & .82 & .95 \\
\hline Social Goads & .81 & .94 & .99 \\
\hline Sensory Processes & .82 & .92 & .96 \\
\hline Tolerance & .84 & .95 & .98 \\
\hline Weight Control & .42 & .80 & .93 \\
\hline
\end{tabular}

\subsubsection{Measures}

The two tasks for this study were the Eriksen Flanker task and the Go/NoGo task which were programmed using OpenSesame (version 3.1.9; Mathôt, Schreij, and Theeuwes 2012). For a brief overview of each task, the trial structures are displayed in Figure 4.1. 
The Eriksen Flanker task (Eriksen and Eriksen 1974) was designed based on Rass et al. (2014). The task assesses how well participants can ignore distracting information and can be considered a measure of attentional control. The task began with a central fixation dot that remained on the screen for a random duration between 500ms and $1000 \mathrm{~ms}$ to deter habitual responding. A string of five letters was then presented in the centre of the screen. The letter strings were either congruent (HHHHH or SSSSS) or incongruent (HHSHH or SSHSS). The participant was required to identify the central letter in the string using the corresponding letters on the keyboard ( $\mathrm{s}$ or $\mathrm{h}$ ). The stimulus remained on the screen until the participant made a response. After the participant made a response, a fixation dot was presented for $1000 \mathrm{~ms}$ either in green if the participant made the correct response, or in red if they made an incorrect response. The next trial then began with a regular white fixation dot.

The task was divided into one practice block and four experimental blocks. The practice block contained 20 trials to familiarise the participant with the task and provide personalised feedback. As it was important for the participant to make some errors, they were informed that their responses were great if their accuracy was between $75 \%$ and $90 \%$. If their accuracy was below $75 \%$, they were informed to be more careful, and if their accuracy was above $90 \%$, they should provide faster responses (Olvek and Hajcak 2009). Each experimental block contained 100 trials with each congruent and incongruent stimulus repeated 25 times in a randomised order. This meant there were 200 congruent and 200 incongruent trials across the task. At the end of each experimental block, the participants were provided with one of the three personalised messages and a 30 second break. This produced a task lasting approximately 20 minutes.

The Go/NoGo task was used based on Rass et al. (2014). The task measures behavioural response inhibition by requiring the participant to make a frequent rapid button press to Go stimuli and withhold a response to infrequent NoGo stimuli (Smith et al. 2014). The task began with a central fixation dot that was presented for 500$1000 \mathrm{~ms}$. An X or an O was then presented in the centre of the screen for $200 \mathrm{~ms}$. When an $\mathrm{X}$ appeared, the participant was required to press the letter $\mathrm{X}$ on the keyboard as quickly as possible. When an $\mathrm{O}$ appeared, the participant was required to withhold 
A

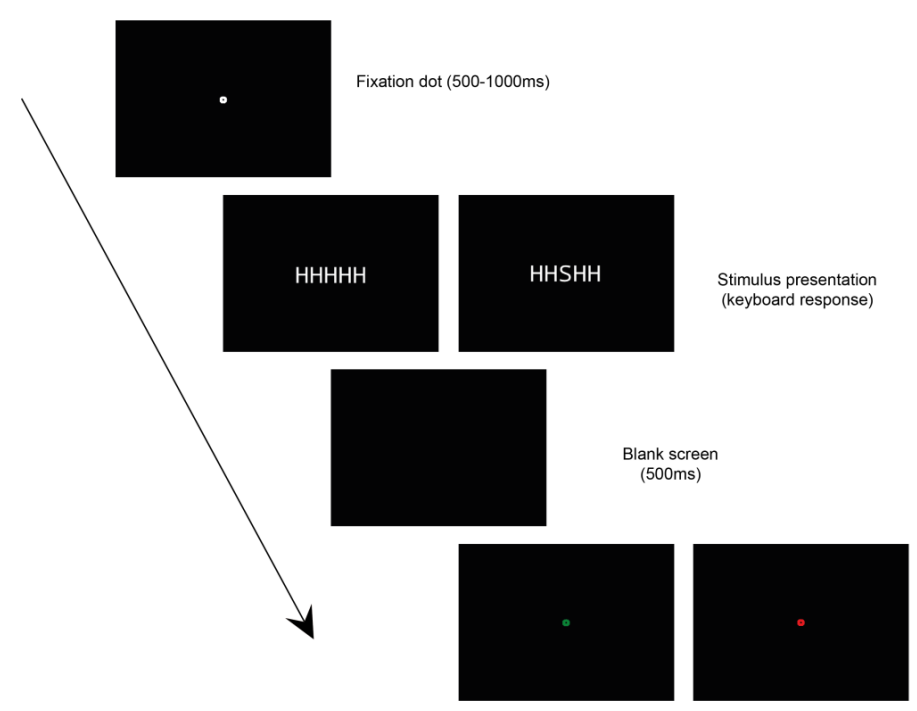

Response feedback (500ms)

B

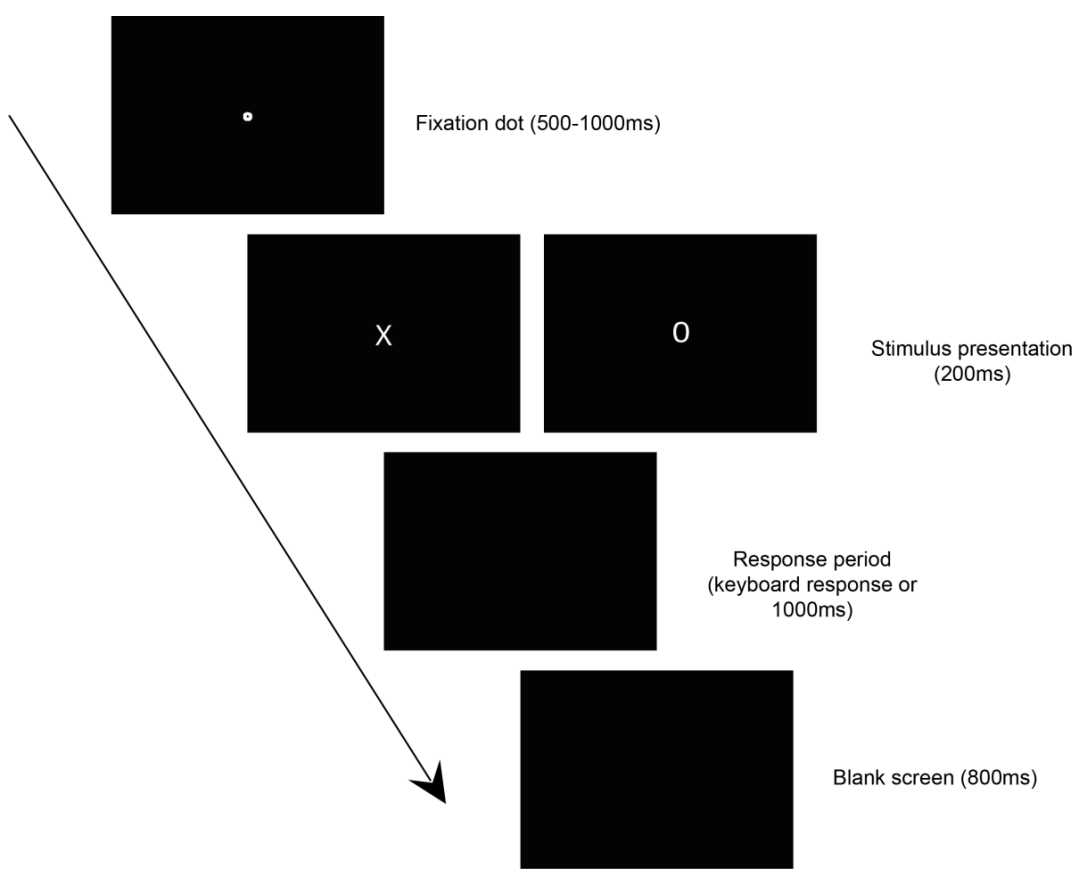

FIGURE 4.1: Overview of Eriksen Flanker (A) and Go/NoGo (B) task. 
making any response. The letter $\mathrm{X}$ appeared frequently (80\% of trials) and the letter O appeared infrequently ( $20 \%$ of trials; Rietdijk et al. 2014). Having infrequent NoGo trials and rapid trial presentation is crucial for creating a pre-potent response, and activity related to inhibitory control is reduced when this is not the case (Wessel 2018). The order of stimuli presentation was randomised for each participant. The participant had $1000 \mathrm{~ms}$ to make a response or withhold a response until timeout. The participant was then displayed a blank screen for $800 \mathrm{~ms}$ regardless of the response. The next trial then began with a white fixation dot.

Similar to the Eriksen Flanker task, the task was divided into one block of practice trials and four experimental blocks. The practice block contained 20 trials and each experimental block contained 100 trials. This meant there were 320 Go trials and 80 NoGo trials across the task. The participant received a 30 second break after each experimental block. This produced a task lasting approximately 20 minutes.

\subsubsection{Procedure}

At the start of the session, all participants read an information letter and provided written informed consent. This study was approved by the Faculty of Health and Life Sciences Ethical Approval board (Reference P61175, see Appendix C). All participants were asked a series of questions to determine if they met all the inclusion criteria. Smoking participants abstained from smoking for an hour before the start of their session. All participants completed a demographics questionnaire and the PANAS at the start of the session. Smokers provided additional information on their smoking habits, and completed a copy of the FTCD and WISDM. All smokers provided a measure of exhaled CO using the MicroCO Monitor, although two measurements were missing due to technical issues with the monitor. Participants were prepared to wear the EEG cap before completing each task in a counterbalanced order across participants. In between each task, participants were provided with a five minute break. When all tasks were completed, the EEG cap was removed before the participant completed a final version of the PANAS (plus QSU-brief for smokers), and finally debriefed. Each session lasted approximately two hours. Participants received research participation credits in exchange for their time. 


\subsubsection{EEG recording and processing}

The electroencephalogram (EEG) was recorded using a Biosemi ActiveTwo measurement system $(1024 \mathrm{~Hz}$ sampling rate). Thirty two electrodes were mounted in a cap using the 10/20 system with four auxiliary electrodes fitted to the outside of each eye, and above and below the left eye. Impedence was maintained below $20 k \Omega$ during the recording for each electrode.

Offline processing was performed using MNE-Python (Gramfort, Luessi and Larson 2013), an open-source software package for EEG data analysis. The Python and R code for the pre-processing pipeline can be found on Github (https://github.com/ BartlettJE/PhD_EEG). The continuous recording was referenced to an average of all the electrodes and filtered with a bandpass of $0.15-30 \mathrm{~Hz}$ (Rietdijk et al. 2014). Ocular correction was performed using Independent Component Analysis (ICA) on the filtered continuous data (Hoffman and Falkenstein 2008), and the components containing artefacts were removed. Epochs were created in 1000ms segments (200ms pre-stimulus and $800 \mathrm{~ms}$ post-stimulus). The data were baseline corrected using the 200ms pre-stimulus period for the Go/NoGo task and the 200ms pre-response period for the Eriksen Flanker task. In each task, epochs with voltages exceeding $\pm 100 \mu \mathrm{V}$ were removed (Rietdijk et al. 2014). Epochs from trials containing incorrect responses were removed (responding to NoGo trials, missing Go trials, pressing the wrong key on the Flanker task).

The minimum number of trials included for each participant was 8 correct and incorrect trials on the Flanker task, and 20 Go and NoGo trials on the Go/NoGo task. These numbers were selected based on research that has evaluated how many trials are required to produce an internally consistent ERP component (Rietdijk et al. 2014; Steele, Anderson and Claus 2016; Boudewyn, Luck, and Farrens 2017). Participants were only excluded from the task where they contributed too few trials. They were retained in the other task if they contributed enough trials to retain as much data as possible. 


\subsubsection{Data analysis}

Data analysis was performed using $\mathrm{R}$. The $\mathrm{R}$ code for the results is available on the OSF project (https://osf .io/yuxmk/). The individual R packages used in each study are provided in Appendix C. For variables that did not meet parametric assumptions, non-parametric tests were performed where appropriate. Any within-subject factors that violated sphericity were adjusted using the Greenhouse-Geisser correction.

\subsection{Results}

\subsubsection{Data screening}

Due to slow data collection stemming from too few smokers signing up, only 21 nonsmokers and 12 smokers completed the study. For smokers, this was lower than the minimum number of participants identified in the pre-registration protocol and power analysis. The plan was to include 18 daily and 18 non-daily smokers per group before performing an interim analysis. This means smokers were divided into smaller sub-groups than specified in the power analysis and therefore the results should be considered exploratory.

\subsubsection{Inhibitory control}

The minimum number of trials in the Go and NoGo conditions in order to be internally consistent was 20. Five non-smokers and two smokers had fewer than 20 trials in one or more conditions, and were not included in the analysis. This left 10 smokers and 16 non-smokers. There were four non-daily and six daily smokers. The mean number of Go trials was 266.00 ( $S D=64.90$; range 124-320) and the mean number of NoGo trials was $51.81(S D=15.22$; range 21-74).

\subsubsection{Error processing}

The minimum number of trials in the correct and incorrect conditions in order to be internally consistent was 8 . Three non-smokers and two smokers had fewer than 8 trials in one or more conditions, and were not included in the analysis. This left 10 smokers and 18 non-smokers. There were four non-daily and six daily smokers. The 
mean number of correct trials was $294.75(S D=77.20$; range 75-374) and the mean number of incorrect trials was $38.36(S D=17.69$; range 12-80).

\subsubsection{Demographics}

The demographics cover the 19 non-smokers and 10 smokers that were included in at least one task. The key demographic characteristics for each smoking group is presented in Table 4.2. Across the whole sample, participants were predominantly white, with the two largest groups identifying themselves as white British (44.83\%: 13) or white European (20.69\%: 6). There were smaller proportions of black British (10.34\%: 3), Asian (6.9\%: 2), Chinese (6.9\%: 2), British Asian (3.45\%: 1), Greek (3.45\%: 1), and Hispanic (3.45\%: 1) participants. Figure 4.2 shows the distribution of the key smoking variables. Despite the small sample sizes, there is a clear distinction between daily and non-daily smokers.

\subsubsection{Inhibitory control}

\subsubsection{Behavioural data}

The behavioural analyses were not outlined in the pre-registration protocol as previous research had not consistently established any effects. Therefore, the behavioural results should also be considered exploratory. For the Go/NoGo task, the DV was the percentage error where participants provided an incorrect response. A $3 \times 2$ mixed ANOVA was performed with one between-subject factor of smoking group (nonsmoker vs daily smokers vs non-daily smoker) and one within-subject factor of trial type (Go vs NoGo).

There was not a significant effect of smoking group $\left(\mathrm{F}(2,26)=1.43, p=.257, \eta G^{2}\right.$ $=.061)$, but there was a significant main effect of trial type $(F(1,26)=46.59, p<.001$, $\left.\eta G^{2}=.425\right)$. Participants had greater percentage error on NoGo trials $(M=21.16 \%$, $S D=12.41 \%)$ than Go trials $(M=3.07 \%, S D=4.98 \%)$. Due to potential issues with normality, this effect was corroborated with a non-parametric equivalent. A Wilcoxon paired samples test showed this result was robust, with higher percentage error on NoGo trials than Go trials, $Z=-4.50, p<.001, r=0.84$. There was not a significant 
TABLE 4.2: Study Three mean $(S D)$ values for participant characteristics and scale scores.

\begin{tabular}{lccc}
\hline & Non-daily smokers & Daily smokers & Non-smokers \\
\hline Age & $20.50(1.29)$ & $21.17(1.33)$ & $24.47(6.49)$ \\
\hline CO $(\mathrm{ppm})^{*}$ & $4.33(5.13)$ & $11.00(3.39)$ & - \\
\hline $\begin{array}{l}\text { Gender } \\
\text { (female:male) }\end{array}$ & $2: 2$ & $3: 3$ & $12: 7$ \\
\hline $\begin{array}{l}\text { PANAS } \\
\text { positive before }\end{array}$ & $36.75(5.38)$ & $28.50(9.46)$ & $29.74(6.76)$ \\
\hline $\begin{array}{l}\text { PANAS } \\
\text { positive after }\end{array}$ & $32.00(12.46)$ & $29.50(9.46)$ & $32.00(12.46)$ \\
\hline $\begin{array}{l}\text { PANAS } \\
\text { negative before }\end{array}$ & $18.25(3.69)$ & $15.17(4.71)$ & $15.63(5.77)$ \\
\hline $\begin{array}{l}\text { PANAS } \\
\text { negative after }\end{array}$ & $16.50(1.91)$ & $18.00(7.80)$ & $12.89(3.16)$ \\
\hline FTCD & $1.00(1.41)$ & $2.83(2.23)$ & - \\
\hline CPD & $2.00(0.82)$ & $10.33(6.15)$ & - \\
\hline WISDM total & $28.90(7.07)$ & $52.31(8.70)$ & - \\
\hline $\begin{array}{l}\text { Last cigarette } \\
\text { (minutes) }\end{array}$ & $900.00(300.00)$ & $133.50(201.75)$ & - \\
\hline Years as a smoker & $3.50(2.65)$ & $4.33(2.34)$ & - \\
\hline $\begin{array}{l}\text { QSU-B } \\
\text { factor 1 before }\end{array}$ & $11.75(2.87)$ & $24.67(6.71)$ & - \\
\hline $\begin{array}{l}\text { QSU-B } \\
\text { factor 1 after }\end{array}$ & $13.00(4.24)$ & $29.33(7.55)$ & - \\
\hline $\begin{array}{l}\text { QSU-B } \\
\text { factor 2 before }\end{array}$ & $5.25(0.50)$ & $13.50(6.19)$ & - \\
\hline $\begin{array}{l}\text { QSU-B } \\
\text { factor 2 after }\end{array}$ & $6.25(1.26)$ & $19.17(7.25)$ & - \\
\hline & & & \\
\hline
\end{tabular}

Abbreviations. CO (Carbon Monoxide); CPD (Cigarettes per day); FTCD (Fagerström Test for Cigarette Dependence); PANAS (Positive and Negative Affect Schedule); QSU-B (Brief Questionnaire of Smoking Urges); WISDM (Wisconsin Inventory for Smoking Dependence Motives).

Note. ${ }^{*}$ The $\mathrm{CO}$ data from one daily and one non-daily smoker was not available. ${ }^{* *}$ Last cigarette data shows median and IQR due to large skew in non-daily smokers. 

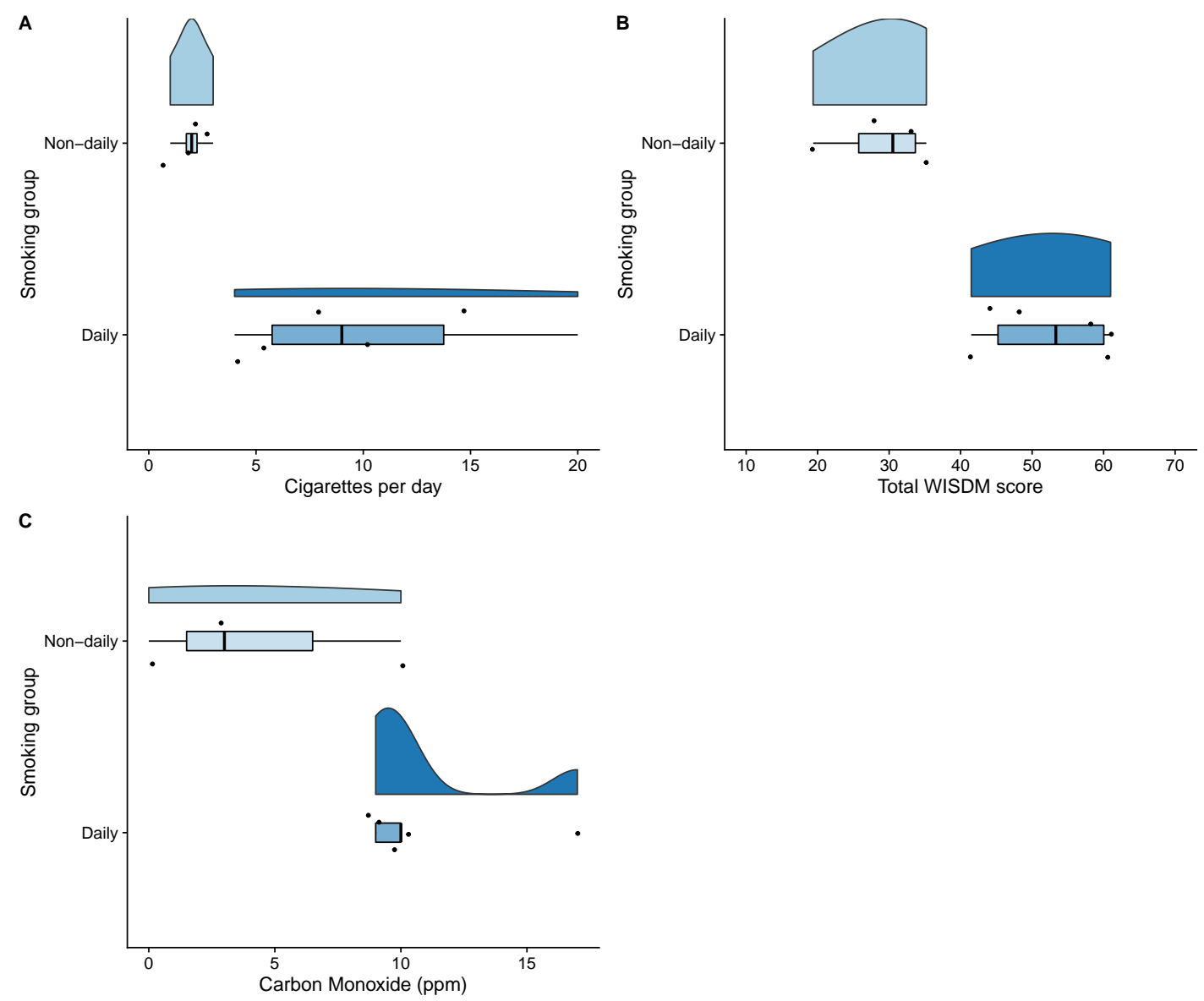

FIGURE 4.2: Three different measures of nicotine dependence: (A) number of cigarettes per day, (B) WISDM total score, and (C) exhaled concentration of $\mathrm{CO}(\mathrm{ppm})$. The data are presented as raincloud plots. The top element for each group represents the distribution of scores through the density. The bottom element presents the individual data points, with a boxplot superimposed to show summary statistics. Note the $\mathrm{x}$-axis of $\mathrm{B}$ ranges from 10-70 due to the range of scores.

interaction between smoking group and trial type, $\mathrm{F}(2,26)=0.19, p=.825, \eta G^{2}=.006$.

The results for the behavioural data are shown graphically in Figure 4.3.

\subsubsection{ERP components}

The DV was the difference in mean amplitude (NoGo - Go) measured in Microvolts $(\mu V)$ with higher absolute values indicating relatively greater NoGo activity in either a positive direction for positive components (P3), or a negative direction for negative components (N2). Figure 4.4 shows the time-locked waveforms for each smoking group split by electrode. A 3 × 3 mixed ANOVA was performed with one betweensubjects IV of smoking group (non-smoker vs daily smokers vs non-daily smoker) and 


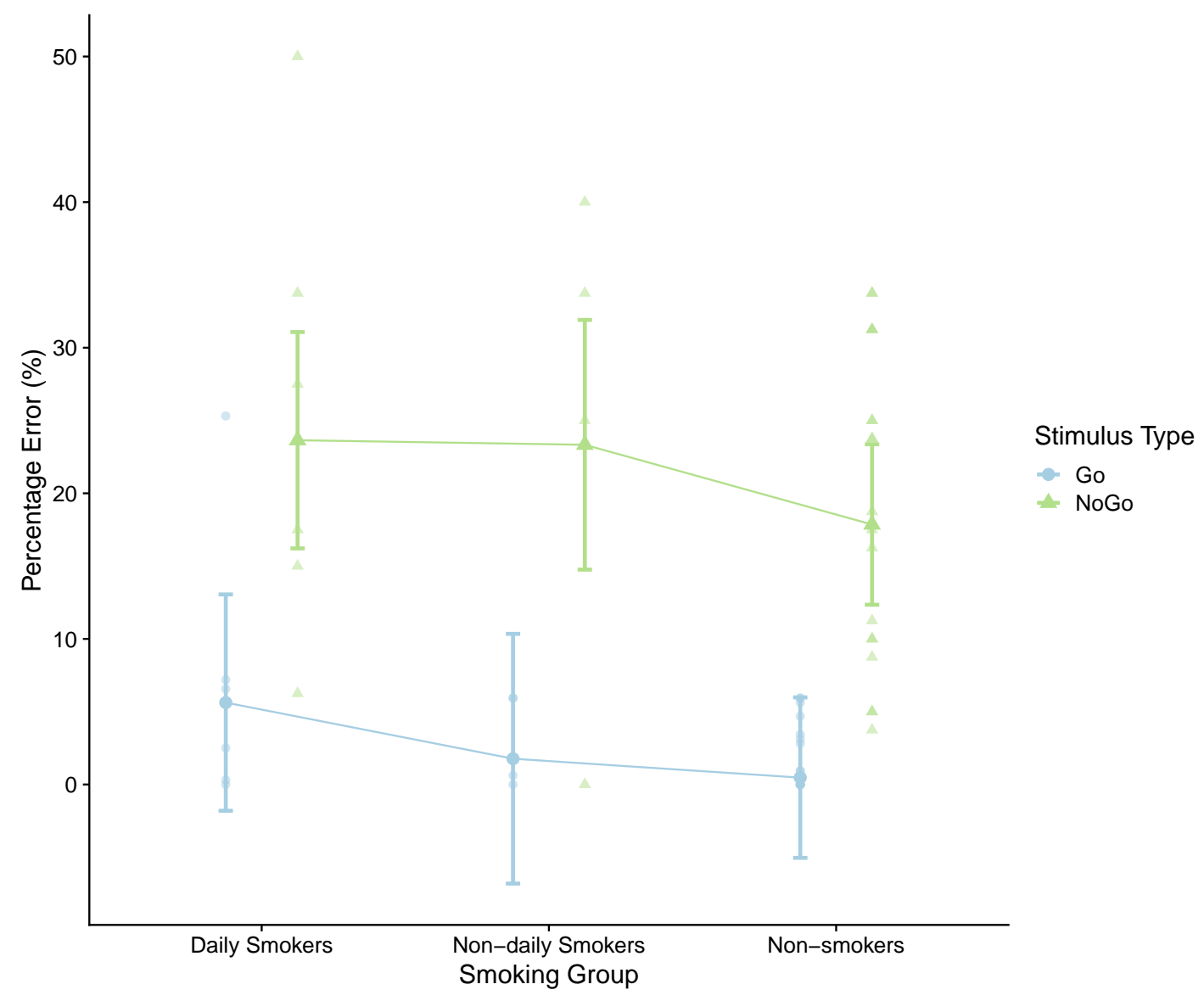

FIGURE 4.3: Interaction plot showing the percentage error (\%) on Go and NoGo trials for each smoking group. The error bars represent the 95\% CI. Individual data points are included semi-transparent in the background.

one within-subjects IV of electrode ( $\mathrm{Cz}$ vs Fz vs Pz). This is highlighted as a deviation from the pre-registration protocol as the original plan was to use a $3 \times 3 \times 2$ mixed ANOVA with an additional factor of trial type (Go vs NoGo). However, given the smaller sample size, removing one factor reduces the increased Type I error rate associated with a larger number of factors and prevents a further reduction in the degrees of freedom (Cramer et al. 2016; Luck and Gaspelin 2017). The degrees of freedom for the main effect of electrode and the interaction effect were corrected using the Greenhouse-Geisser correction.

For the N2 time window, there was not a significant main effect of smoking group $\left(\mathrm{F}(2,23)=0.29, p=.748, \eta G^{2}=.011\right)$ or electrode $\left(\mathrm{F}(1.73,39.77)=0.48, p=.595, \eta G^{2}\right.$ $=.012)$. There was also no interaction between smoking group and electrode $(\mathrm{F}(3.46$, $\left.39.77)=1.59, p=.203, \eta G^{2}=.074\right)$. For the P3 time window, there was no main effect of 

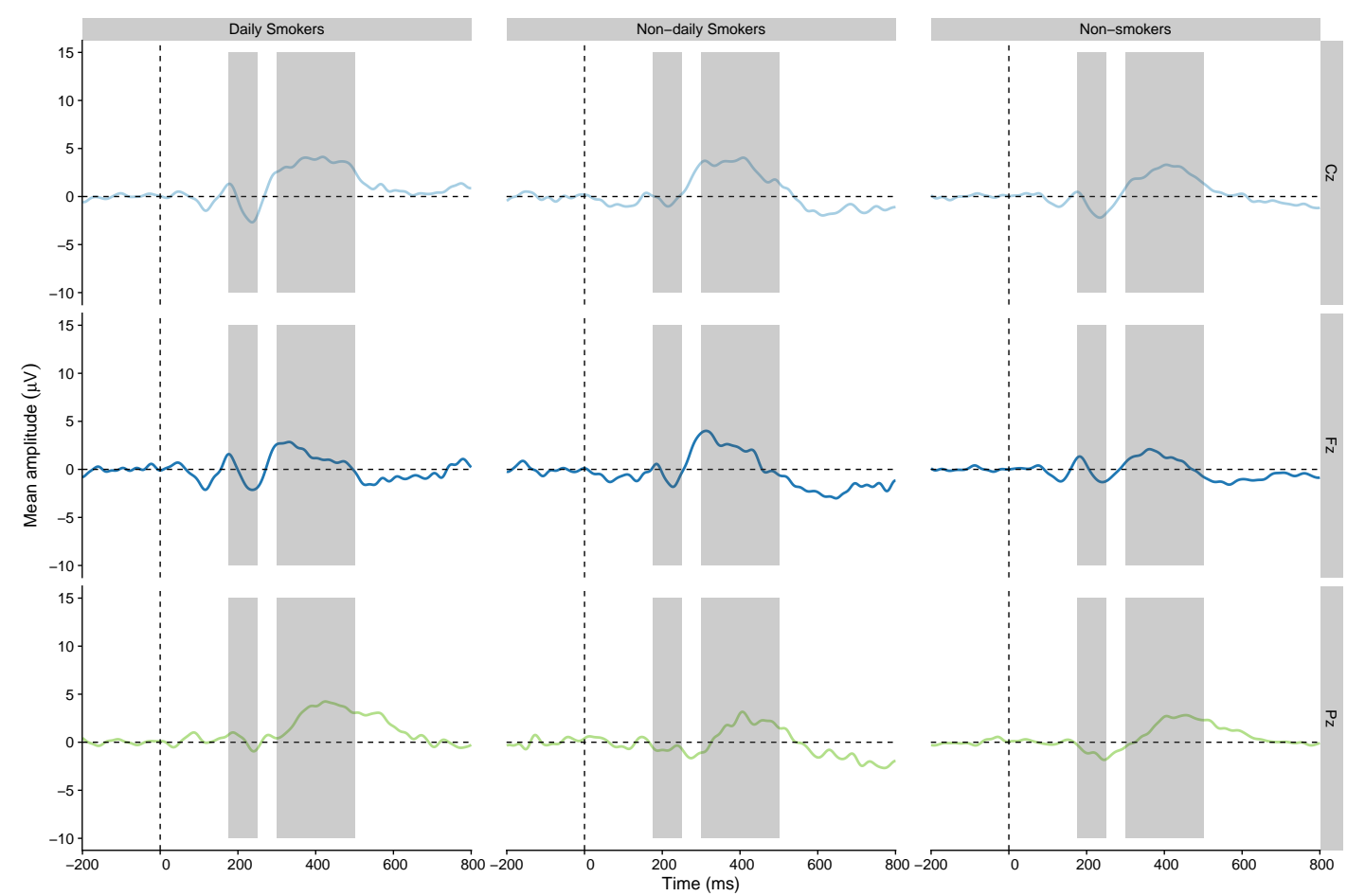

FIGURE 4.4: Grand average waveform for the difference score (NoGo $\mathrm{Go})$ in mean amplitude $(\mu V)$. The grey boxes show the time windows of interest for each component. The N2 is the first box between $175-250 \mathrm{~ms}$ and the P3 is the second box between $300-500 \mathrm{~ms}$.

smoking group $\left(\mathrm{F}(2,23)=1.12, p=.342, \eta G^{2}=.053\right)$, but there was a significant effect of electrode $\left(\mathrm{F}(1.99,45.81)=5.62, p=.007, \eta G^{2}=.094\right)$. The pairwise comparisons were not followed up as a main effect of electrode was not of interest. There was no significant interaction between smoking group and electrode $(\mathrm{F}(3.98,45.81)=0.55, p$ $\left.=.697, \eta G^{2}=.020\right)$. The mean amplitude in each component per smoking group and electrode is displayed in Figure 4.5.

\subsubsection{Error processing}

\subsubsection{Behavioural data}

For the Eriksen Flanker task, the DV was the percentage error where participants provided the incorrect response. A $3 \times 2$ mixed ANOVA was performed with one between-subjects IV of smoking group (non-smoker vs daily smokers vs non-daily smoker) and one within-subjects IV of congruency (congruent vs incongruent). These results should also be considered exploratory as they were not outlined in the preregistration protocol. 

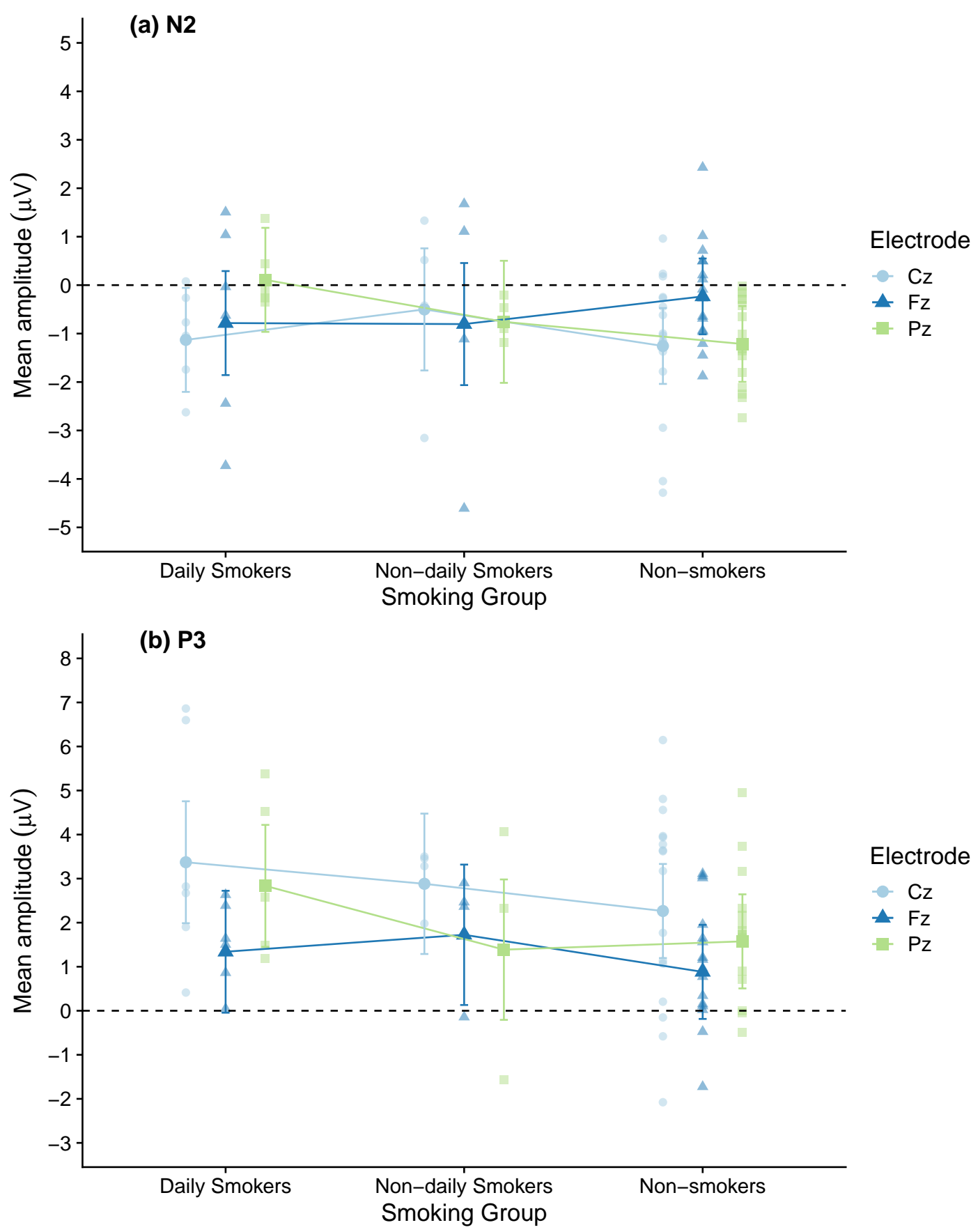

FIGURE 4.5: Interaction plot showing the difference in mean amplitude (NoGo - Go) for each smoking group and electrode. Panel A shows the N2 component and Panel B shows the P3 component. The error bars represent the $95 \%$ CI. Individual data points are included semitransparent in the background.

There was not a significant effect of smoking group $\left(\mathrm{F}(2,25)=2.38, p=.113, \eta G^{2}=\right.$ $.101)$, but there was a significant main effect of congruency $(\mathrm{F}(1,25)=53.52, p<.001$, $\left.\eta G^{2}=.468\right)$, with participants having greater percentage error on incongruent $(M=$ $21.62 \%, S D=6.23 \%)$ trials than congruent $(M=10.02 \%, S D=4.25 \%)$ trials. A Wilcoxon 
paired samples test showed this result was robust to minor violations of parametric assumptions, with higher percentage error on incongruent trials than congruent trials, $\mathrm{Z}=-4.54, p<.001, r=0.86$. There was not a significant interaction between smoking group and congruency, $\mathrm{F}(2,25)=0.09, p=.915, \eta G^{2}=.003$. The results for the behavioural data are shown graphically in Figure 4.6.

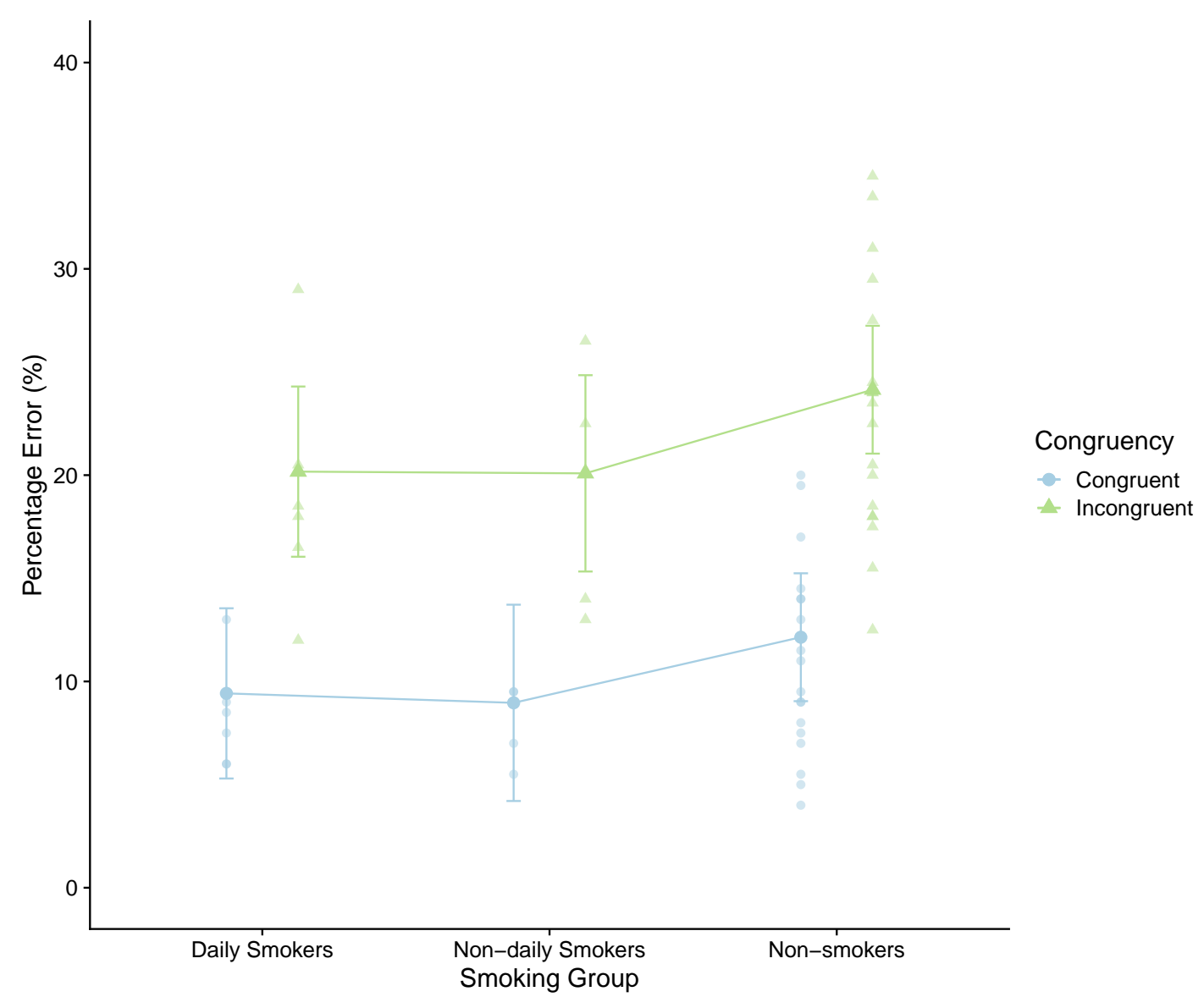

FIGURE 4.6: Interaction plot showing the percentage error (\%) on congruent and incongruent trials for each smoking group. The error bars represent the $95 \%$ CI. Individual data points are included semitransparent in the background.

\subsubsection{ERP components}

A similar change in analysis in comparison to the pre-registration protocol was performed for the Eriksen Flanker task. The DV was the difference in mean amplitude $(\mu V)$ between correct and incorrect trials (incorrect - correct), with higher absolute values indicating relatively greater incorrect activity in either a positive direction for 
positive components (Pe), or a negative direction for negative components (ERN). Figure 4.7 shows the time-locked waveforms for each smoking group split by electrode. A $3 \times 3$ mixed ANOVA was performed with one between-subjects IV of smoking group (non-smoker vs daily smokers vs non-daily smoker) and one within-subjects IV of electrode ( $\mathrm{Cz}$ vs Fz vs $\mathrm{Pz}$ ). The deviation from the pre-registration protocol also applies here. The degrees of freedom for the main effect of electrode and the interaction effect were corrected using the Greenhouse-Geisser correction.
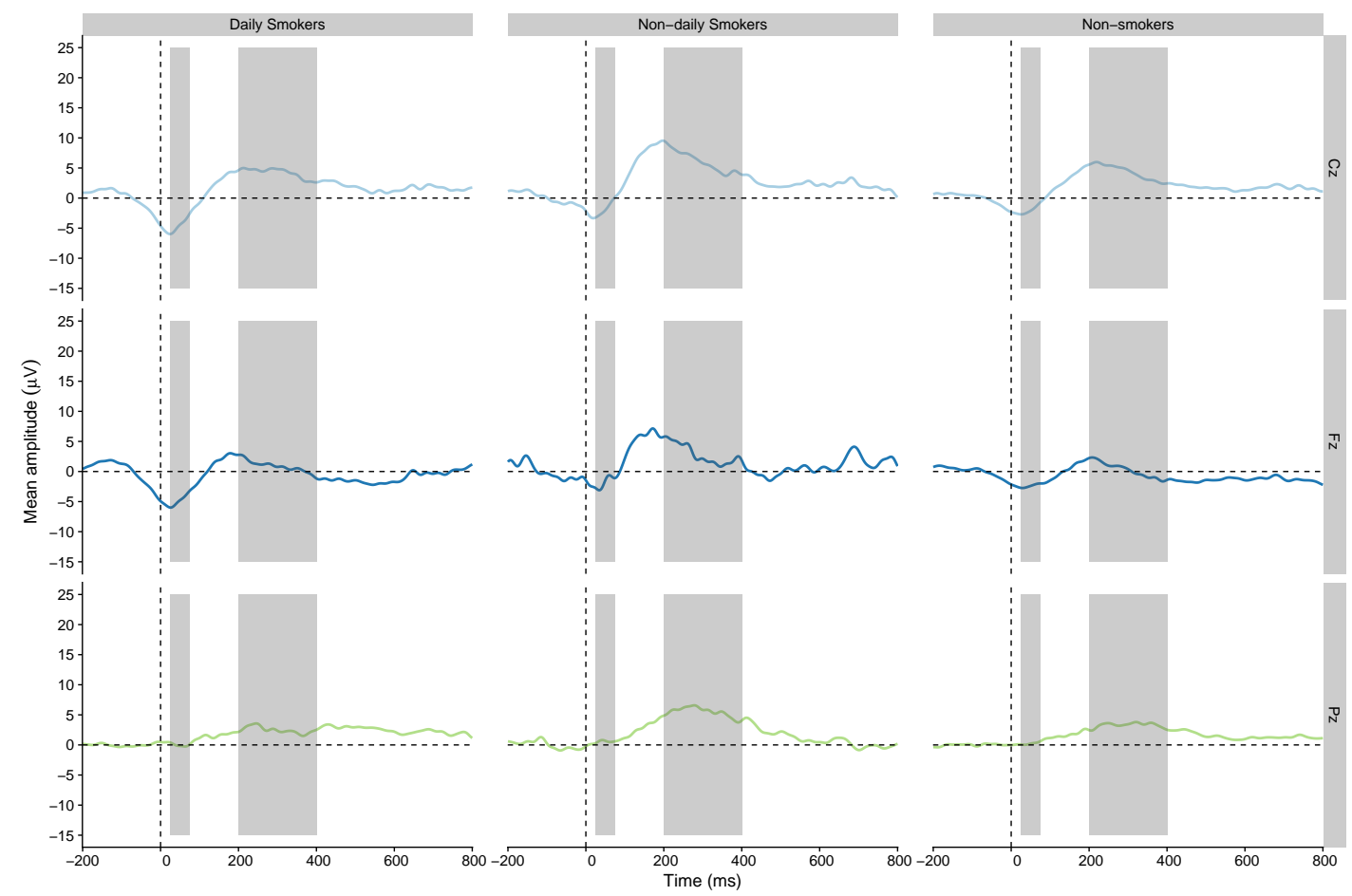

FIGURE 4.7: Grand average waveform for the difference score (Incorrect - Correct) in mean amplitude $(\mu V)$. The grey boxes show the time windows of interest for each component. The ERN is the first box between $25-75 \mathrm{~ms}$ and the Pe is the second box between $200-400 \mathrm{~ms}$.

For the ERN time window, there was not a significant effect of smoking group (F $\left.(2,25)=3.27, p=.055, \eta G^{2}=.122\right)$, but there was a main effect of electrode (F (1.91, $47.77)=19.45, p<.001, \eta G^{2}=.268$ ). The pairwise comparisons were not followed up as a main effect of electrode was not of interest. There was not a significant interaction between smoking groups and electrode $\left(\mathrm{F}(3.82,47.77)=1.17, p=.336, \eta G^{2}=.042\right)$. For the Pe time window, there was not a significant main effect of smoking group $(\mathrm{F}(2$, $\left.25)=2.29, p=.122, \eta G^{2}=.090\right)$, but there was a main effect of electrode $(\mathrm{F}(1.54,38.48)$ $\left.=13.08, p<.001, \eta G^{2}=.195\right)$. There was no significant interaction between smoking 
group and electrode, $\mathrm{F}(3.08,38.48)=0.30, p=.827, \eta G^{2}=.011$. The mean amplitude in each component per smoking group and electrode is displayed in Figure 4.8.

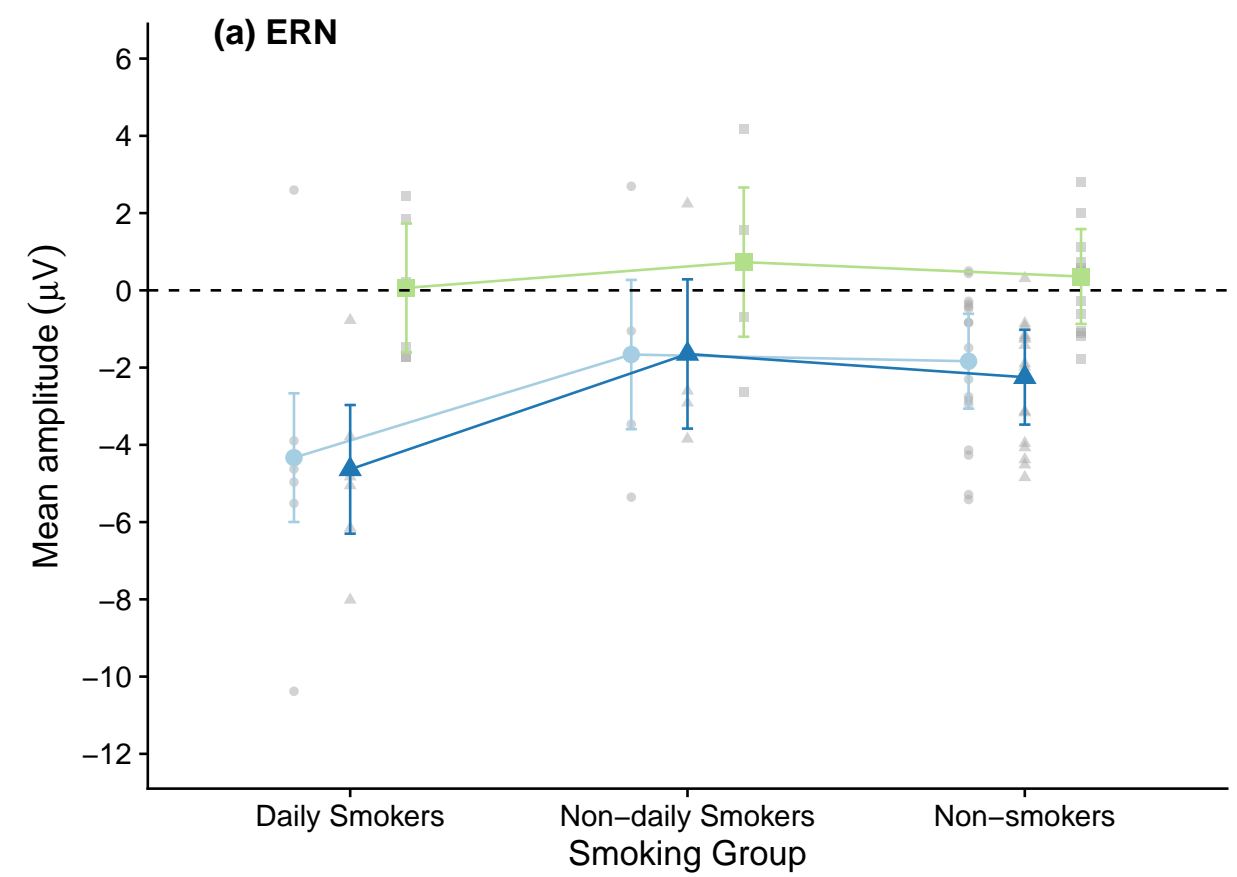

Electrode

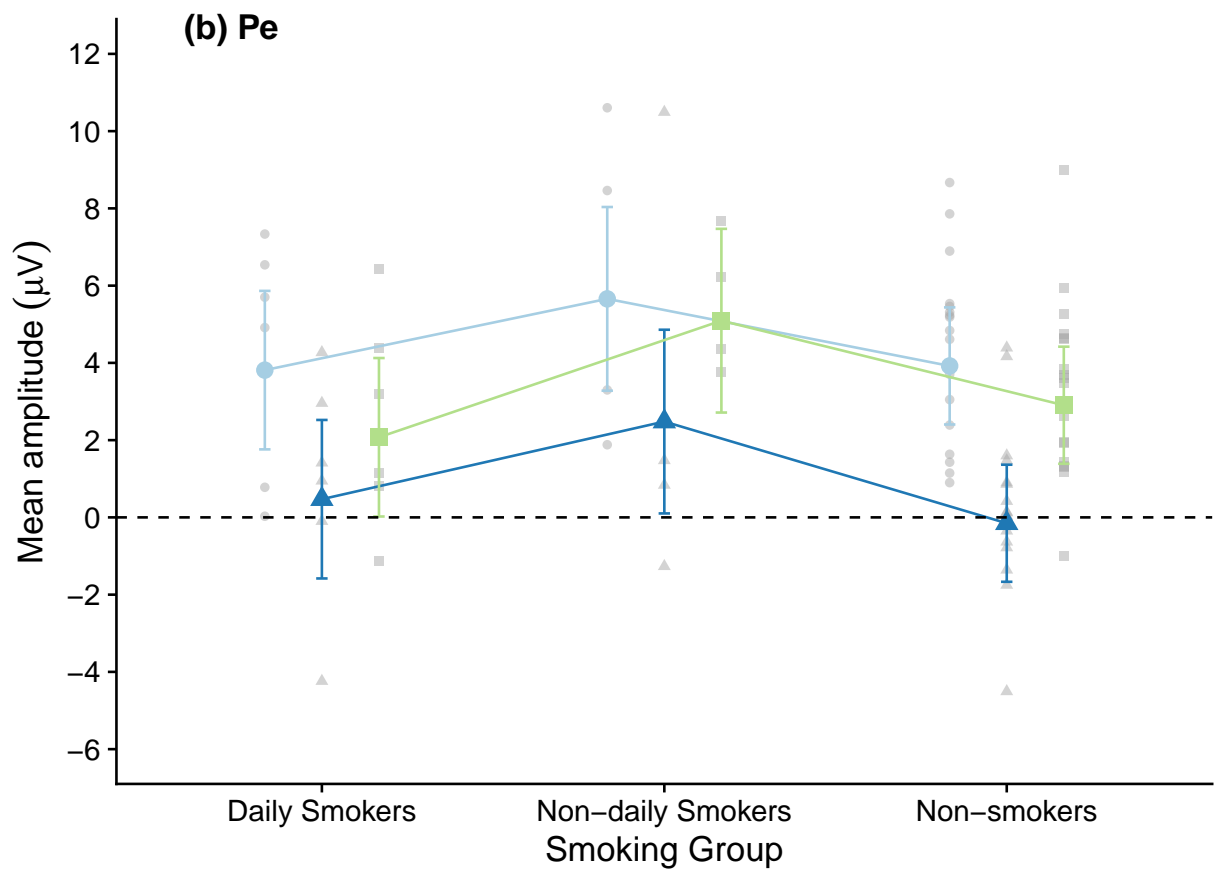

Electrode

- $\mathrm{Cz}$

$\pm \mathrm{Fz}$

- $\mathrm{Pz}$

FIGURE 4.8: Interaction plot showing the difference in mean amplitude (Incorrect - Correct) for each smoking group and electrode. Panel A shows the ERN component and Panel B shows the Pe component. The error bars represent the $95 \%$ CI. Individual data points are included semi-transparent in the background. 


\subsubsection{Reliability estimates}

In order to estimate the reliability of the measures, the permutation based split-half reliability was calculated (Parsons et al. 2019). The procedure for preparing the data followed Rietdijk et al. (2014) where the mean amplitude per component was calculated for each trial in the task.

For the Eriksen Flanker task, this meant calculating the mean amplitude between 25-75ms post-response for the ERN and between 200-400ms post-response for the Pe. Only the incorrect trials were included where the amplitude was expected to be larger. This left a mean amplitude per each valid trial from the processing steps followed for the main analyses. The splithalf $\mathrm{R}$ package was used to calculate split-half reliability using 5000 permutations. For the ERN component, there was moderate to high splithalf reliability for the $\mathrm{Fz}(r=.84,95 \% \mathrm{CI}=[.73, .92]), \mathrm{Pz}(r=.71,95 \% \mathrm{CI}=[.51, .86])$, and Cz electrodes $(r=.90,95 \% \mathrm{CI}=[.82, .95])$. For the Pe component, there was high splithalf reliability for the $\mathrm{Fz}(r=.91,95 \% \mathrm{CI}=[.85, .96]), \mathrm{Pz}(r=.91,95 \% \mathrm{CI}=[.86, .95])$, and $\mathrm{Cz}$ electrodes $(r=.91,95 \% \mathrm{CI}=[.83, .96])$. As in Chapter Three, these estimates were corrected using the Spearman-Brown formula (Parsons et al. 2019).

For the Go/NoGo task, the mean amplitude per trial was calculated between 175250ms for the N2 component and 300-500ms for the P3 component. Consistent with Rietdijk et al. (2014), the analysis focused on NoGo trials. For the N2 component, there was moderate to high split-half reliability for the $\mathrm{Fz}(r=.83,95 \% \mathrm{CI}=[.71, .92]), \mathrm{Pz}(r$ $=.74,95 \% \mathrm{CI}=[.58, .86])$, and $\mathrm{Cz}$ electrodes $(r=.84,95 \% \mathrm{CI}=[.71, .92])$. For the P3 component, there was high split-half reliability for the $\mathrm{Fz}(r=.83,95 \% \mathrm{CI}=[.70, .92])$, $\mathrm{Pz}(r=.89,95 \% \mathrm{CI}=[.82, .95])$, and $\mathrm{Cz}$ electrodes $(r=0.95,95 \% \mathrm{CI}=[.91, .97])$.

\subsection{Discussion}

The hypotheses for this study were that daily smokers were expected to show a smaller mean amplitude in comparison to non-daily smokers, who were expected to show a smaller mean amplitude than non-smokers. This pattern of results was predicted for both the Go/NoGo and Eriksen Flanker task, and each ERP component associated with these. There were no significant effects involving smoking group, nor any significant interactions between electrode and smoking group. There was a significant 
effect of electrode for the P3 component during the Go/NoGo task, and for both the ERN and Pe component during the Eriksen Flanker task. This suggests the mean amplitude differed across electrodes, but this was not followed up as it was not an effect of interest without interacting with the smoking group.

Previous research consistently found that smokers displayed deficits in ERP measures of inhibitory control and error processing. Smokers had lower N2 (Luijten et al. 2011a; Buzzell et al. 2014) and P3 (Anokhin et al. 2000; Evans et al. 2009) amplitude in comparison to non-smokers using inhibitory control measures. Similarly, smokers had lower ERN (Franken et al. 2010; Luijten et al. 2011b) and Pe (Luijten et al. 2011b) amplitude than non-smokers in error processing measures. Although studies did not consistently find lower amplitudes in each ERP component, there was usually at least one. The rationale for this study was there were three studies that explored inhibitory control in idiosyncratic ways and only one known study had explored error processing in daily and non-daily smokers. Rass et al. (2014) explored error processing using a Go/NoGo and Eriksen Flanker task, finding that intermittent smokers had greater Pe amplitude than daily smokers. However, no study had looked at ERP components associated with inhibitory control using a speeded response-time task. This study was the first known study to explore these mechanisms in daily and non-daily smokers, but all the effects of interest were non-significant. The main caveat here is the sample size prevents any firm conclusions.

The sample size of the smokers was lower than planned in the pre-registration protocol due to difficulties collecting a sufficient number of daily and non-daily smokers. A sensitivity power analysis suggests that to test the prediction that daily smokers had a significantly lower mean amplitude than non-daily smokers, this would only be sensitive enough to detect effects of Cohen's $\mathrm{d}=1.76$ or larger (power $=.80$, alpha $=.05$, one-tailed). Even the comparison with the largest collective sample size - testing for the effect of a smaller mean amplitude in non-daily smokers than non-smokers in the Eriksen Flanker task - would only be sensitive enough to detect effects of Cohen's d = 1.38 or larger. In a large analysis of cognitive neuroscience articles, the median effect size for significant pairwise comparisons was lower than these values $(d=0.93)$, and much lower for non-significant effects ( $d=0.24$; Szucs and Ioannidis 2017). Although this study did not break these estimates down by method to specify EEG research, 
the current study did not have enough statistical power to detect realistic effect sizes for cognitive neuroscience research. In order to detect these effects, future research should include a sufficiently large sample in each sub-group of interest.

The behavioural data for each task was analysed to explore whether there was a difference in error rates between smokers and non-smokers. This was not included in the pre-registration protocol as previous research had not consistently observed an effect between smoking groups. In a meta-analysis, Conti et al. (2019) reported that there was not a significant difference between smokers and non-smokers in four studies focusing on motor impulsivity. However, no study used the Go/NoGo task. In this study, there was a significantly higher error rate on NoGo trials than Go trials in the Go/NoGo task. Similarly, there was a significantly higher error rate on incongruent trials than congruent trials in the Eriksen Flanker task. Both of these effects were large and are consistent with previous research showing that cognitive tasks produce highly consistent effects (Hedge et al. 2017), with the differences between conditions replicating even when participants are told what to expect (Zwaan et al. 2017). In isolation, this effect shows that the tasks worked as intended as all participants produced more errors when faced with incongruent stimuli or stimuli requiring the inhibition of a prepotent response. There was not an interaction with smoking group to suggest one group had a lower or higher error rate. The same caveat applies here as to the ERP results as the sample size in each group was too small to detect realistic effect sizes. Therefore, this would need to be followed-up in future research ensuring a sample size with the ability to detect effect sizes of interest.

In future research, it would be important to establish whether neurocognitive deficits in smokers relative to non-smokers actually means a performance deficit. Previous research did not focus on effect sizes or normative levels of performance to comment on whether the deficits observed in smokers is problematic. Brinkmeyer et al. (2011) provided normative levels of P50 gating amplitude for non-smoking control participants. However, in the manuscript, the discussion did not focus on how much smokers deviated from non-smokers, just that there was a significant difference. The sample size in Study Three was too small to establish normative levels, but future research using ERP measures could learn from other areas of interest. Turetsky et al. (2009) explored deficits in information processing in patients with schizophrenia. Similar to smoking, 
schizophrenia is associated with deficits in neurocognitive mechanisms. Rather than simply compare the performance of schizophrenia patients relative to controls, Turetsky et al. (2009) established a normative level of performance in healthy controls and converted measures such as P3 amplitude in response to auditory oddball tones to z-scores. Schizophrenic patients were on average 0.5 standard deviations worse than the normative levels set by controls. Framing deficits in neurocognitive mechanisms in this way treats the findings like psychometric testing with known distributions of scores, but this level of insight does not appear to have been explored in smoking research. Future studies could focus more on effect sizes and establish whether cognitive control deficits reach an established threshold linked to behavioural impairments, rather than just concluding smokers have lower ERP amplitude than non-smokers and assuming this relates to behavioural problems.

One of the positive findings from this study is the split-half reliability for both tasks across all electrodes ranged from moderate to very high, with the lowest estimate being $r=.71$. Given that estimates of .50 to .75 represent moderate reliability (Koo and Li 2016), the lowest estimate in the study was near the upper bound. This suggests that ERP measures may be best suited for investigating deficits in inhibitory control and error processing. Psychophysiology research has been more scrupulous with establishing the psychometric properties of their measures, with a succession of articles demonstrating the internal consistency of different ERP components including the N2, P3, ERN, and Pe (e.g., Olvet and Hajcak 2009; Cassidy, Robertson, and O'Connell 2012; Clayson and Larson 2013; Rietdijk et al. 2014). Psychology in general has been criticised for not reporting the psychometric properties of the cognitive tasks they use (Hedge et al. 2017; Parsons et al. 2019). The measures used in this study were consistent with previous psychophysiology research as each ERP component was sufficiently stable. This is important as lower internal consistency leads to lower statistical power (Henson 2001). Poor internal consistency would have compounded the issue with power from the smaller than anticipated sample size. As ERP measures show high levels of internal consistency, they are better placed to be used in identifying those at risk of relapse or evaluating the success of cessation interventions. EEG recording is a more practical solution for investigating cessation outcomes as they are relatively inexpensive and mobile in comparison to MRI scanners (Parvaz 
et al. 2011; Houston and Schlienz 2018). Although response time measures would be the most resource friendly, they do not have sufficiently robust psychometric properties (Hedge et al. 2017; Parsons et al. 2019). Study Three demonstrated that the ERP components associated with inhibitory control and error processing are sufficiently stable, and based on previous research, they may be best suited as predictors or markers of cessation outcomes as they have sufficiently high internal consistency.

An interesting observation was the later components in each task ( $\mathrm{P} 3$ and $\mathrm{Pe}$ ) had consistently higher reliability than earlier components (N2 and ERN). This may be due to later components containing more timepoints in their estimates. In earlier components the time windows spanned fewer than $100 \mathrm{~ms}$, whereas later components both had time windows of $200 \mathrm{~ms}$. Using the mean amplitude is less sensitive to noise than the peak amplitude (Luck 2014), and increasing the number of time points included in the estimate increases the signal to noise ratio (Thigpen, Kappenman, and Keil 2017). This means components with larger time windows have more noise averaged out and the underlying signal should be more consistent if it is present. However, there is a limit to this increase. There appears to be a sweet spot for the number of timepoints included in the average, as beyond a point the reliability will begin to decrease. This is because the time window should include the component of interest (Thigpen et al. 2017). If the time window starts to include multiple components, especially when the components have different polarities, then the estimate will not be as stable across trials.

One psychometric property that could not be explored in this study was test-retest reliability. This study showed that components associated with inhibitory control and error processing were moderately to highly consistent across trials within one measure. The measures should also be consistent across multiple measurements to gauge the success of cessation interventions. In order to be confident a change in ERP component is associated with an effective intervention, the measurement of that ERP should be consistent across measurements, or a change in amplitude could be due to the instability of the measure rather than the effectiveness of the intervention. Previous research has largely shown this to be the case. The ERN has strong test-retest reliability after two weeks (Larson et al. 2010) and both the ERN and Pe are reliable after one month (Cassidy et al. 2012). Over long periods, The ERN and Pe are stable across 
four different measurements (Ip et al. 2018), and moderately stable up to 2.5 years (Weinberg and Hajcak 2011). For components associated with inhibitory control, the N2 (Clayson and Larson 2013) and P3 (Hämmerer et al. 2013) has strong test-retest reliability after two weeks. Together, this body of evidence suggests ERP components associated with inhibitory control and error processing are both internally consistent and consistent across time. This means they are best placed to be used in intervention work as they are stable enough within and across sessions to be able to detect any changes associated with an intervention.

When designing this study, there was a choice over different methods. The tasks were modelled closely to those used by Rass et al. (2014) as that was the only previous study to investigate cognitive control mechanisms using speeded response-time tasks in daily and non-daily smokers. The Go/NoGo task was intended to be a "pure" measure of inhibitory control. In this configuration, a prepotent motor response is created by an infrequent NoGo stimulus, where the participants must inhibit a response after it has been initiated (Wessel 2018). However, this is not the only design of the Go/NoGo task. Most previous research used a similar Go/NoGo task where the NoGo trial is created by a consistent infrequent stimulus (Buzzell et al. 2014; Luijten et al. 2011a; Rass et al. 2014). Other research used a version of the task which requires an element of working memory. For example, Evans et al. (2009) presented a series of y or $x$ letters, and the NoGo trial is signalled when the current trial used a different letter to the preceding trial. This involves an aspect of working memory as the participant must retain the previous stimulus before recognising whether the current stimulus is the same or different, and only then can the decision to inhibit the response be made.

These two versions have been compared in an fMRI study with both tasks providing similar patterns of activation during Go trials, but diverging results during NoGo trials (Mostofsky et al. 2003). The pre-supplementary motor area was more active during NoGo trials for both task versions, but working memory tasks which involve counting the stimuli also cause activation in the dorsolateral prefrontal cortex. This is also reflected in ERPs as there does not appear to be any clear differences between the tasks for the $\mathrm{N} 2$ component, but the traditional version led to greater P3 activity in comparison to the counting version (Smith et al. 2013). This suggests the counting version of the Go/NoGo task is not a pure measure of inhibitory control, but requires 
extra working memory resources. This also means the working memory capacity of participants would need to be taken into consideration. When a counting version of the Go/NoGo task was used, participants with a lower working memory capacity had lower accuracy rates than those with higher working memory capacity on trials which required inhibiting a response based on the preceding trial (Redick et al. 2011). On traditional versions of the task, there was a much smaller and non-significant effect between groups differing in working memory capacity. This means it is important to design the task carefully and consider whether you want a pure measure of inhibitory control or the additional influence of working memory. This has the potential to complicate findings in two ways. First, EEG has relatively poor spatial resolution (Light et al. 2010). The activity detected by EEG recordings is the result of activity from several regions, causing a smearing effect (Burle et al. 2015). If you have two studies that use a different design of the task, this could produce different patterns of activation in frontal electrodes as there is greater activity from both the pre-supplementary motor area and dorsolateral prefrontal cortex. Second, if one group of participants has a lower working memory capacity, this could present a confounding variable as this may lead to a difference between Go and NoGo trials, rather than a deficit associated with smoking groups. Fortunately, most previous research has consistently used the traditional version of the task, rather than a counting version, reducing the probability of this being a confound.

Although the sample size limited the conclusions that could be made, there were several strengths to this study surrounding the methods. A number of recommendations have been outlined for improving the rigour of psychophysiology research (Baldwin 2017). First, the Go/NoGo task had an 80:20 split between Go and NoGo trials. Presenting the stimuli rapidly and creating a prepotent response by having $20 \%$ or fewer NoGo trials is crucial for eliciting an inhibitory process (Wessel 2018). A large number of articles use a 50:50 split between trials, meaning the participant just has to decide whether to respond or not, not inhibit a response that has already begun to be initiated. Second, the time windows used for the components were preregistered before data collection. This was important for preventing potential falsepositives, although the small sample size reduced the chance of this happening. Luck and Gaspelin (2017) demonstrated that hand picking a time window based on the 
observed waveforms and choosing the largest electrodes allows you to find bogus effects between two conditions when the trials are randomly selected, rather than reflect real underlying processes. Third, the minimum number of trials included in each condition was supported by previous research on the number of trials required for internally consistent estimates (e.g., Rietdijk et al. 2014). Although this led to some participants being excluded for not having enough trials, it ensured the components could be reliably observed across trials for each eligible participant.

In addition to the smaller than planned sample size, a further limitation should be acknowledged. The location for data collection did not take place in a room specially designed for EEG research. This was due to room availability and being able to keep the EEG equipment consistently in one place. In order to ensure high data quality, the testing environment should be carefully controlled for electrical interference, temperature, and humidity, or this can lead to higher impedance which affects statistical power (Kappenman and Luck 2010). Although using the mean amplitude is more robust to increased levels of background noise (Clayson, Baldwin, and Larson 2013), fewer trials and participants may have been discarded if a room designed for EEG research was used.

\subsubsection{Conclusion}

The aim of this study was to replicate and extend previous research on cognitive control in daily and non-daily smokers. A number of articles found that smokers showed deficits - demonstrated by a lower mean amplitude in ERP components associated with inhibitory control and error processing - in comparison to non-smokers. However, only one known study had directly compared daily and non-daily smokers on error processing, and previous research on inhibitory control had not used a speeded response-time task. This study found no significant effects across smoking group, and no significant interactions between smoking group and electrode. There was a large caveat of the sample size of the two smoking groups being substantially lower than outlined in the pre-registration protocol due to issues with data collection. This had implications on statistical power which meant the study was only sensitive to detect effects far larger than what can reasonably be expected in cognitive neuroscience research. This means future research should repeat this study, but have the resources 
necessary to collect a sufficient sample of daily and non-daily smokers.

The main positive of this research is the focus on measurement. The pre-registration protocol outlined the number of eligible trials across conditions in each participant for them to be included in the analyses. This was based on previous research which outlined the number of trials necessary for an internally consistent ERP measure for inhibitory control and error processing. Each ERP component in this study had moderate to high internal consistency. This means the ERPs associated with these cognitive control mechanisms could be promising in their uses for identifying smokers at risk of relapse or evaluating the effectiveness of an intervention.

Chapter Five presents a general discussion of the research presented in this thesis to integrate the findings of the studies and explore the implications, identify overall strengths and limitations, and outline directions for future research. 


\section{Chapter 5}

\section{General Discussion}

\subsection{Introduction}

In neurocognitive models of addiction such as iRISA (Goldstein and Volkow 2002; 2011; Zilverstand et al. 2018), addictive behaviour is modelled as a syndrome of two complementary processes: overactive drive mechanisms which stimulate behaviour and under-active control mechanisms which monitor and adapt behaviour. These mechanisms could be important for translating the findings of neuroscience research in the lab to improving outcomes in the clinic (Franken and van der Wetering 2015). The systematic review in Chapter Two highlighted that when focusing on these neurocognitive mechanisms in daily and non-daily smokers, research produced either conflicting results or received little empirical attention. Historically, the majority of research focused on comparing drug dependent users to non-users. However, it is important to focus on daily and non-daily smokers as they show similar long-term relapse rates (Tindle and Shiffman 2011; Kotz et al. 2012; Herbec et al. 2014) despite fundamentally different habits and motives (Shiffman et al. 2012a; 2014b). If drive and control processes are associated with addictive behaviour, then it is important to explore how these different smoking groups differ (or not) on these processes.

In this final chapter, the three main contributions of this thesis and their implications will be presented. First, the systematic review in Chapter Two highlighted how there is a problematic level of heterogeneity in the labels and criteria used to define different smoking groups. Second, the findings of Studies One and Two suggest that trait-level differences in attentional bias are at best much smaller than reported in previous research. Finally, Study Three found that the internal consistency of ERP 
components associated with inhibitory control and error processing was moderate to high. Importantly, this was a secondary aim of Study Three. The sample size was smaller than anticipated, meaning no meaningful conclusions could be made about differences in inhibitory control and error processing in daily and non-daily smokers. The general limitations of this thesis will then be outlined before directions for future research. The chapter will then end on a general conclusion summarising the contribution of this thesis to the field of addictive behaviour.

\subsection{Heterogeneity of terminology for smoking groups}

The first important finding in this thesis was highlighting the heterogeneity of labels and criteria used to define smoking groups. The systematic review in Chapter Two provided the starting point by reviewing previous research focusing on neurocognitive mechanisms in daily and non-daily smokers. This was the first known systematic review focusing on these neurocognitive mechanisms in different smoking groups. There were 37 unique combinations of smoking labels and criteria, almost as many combinations as the 42 studies included in the review. In isolation, there were 17 different labels and 32 different definitions. One study referring to "light" and "heavy" smokers may have used very different criteria to another studying reporting to use "light" and "heavy" smokers. This degree of heterogeneity is problematic as it is difficult to directly compare the results of different studies. The most common definition used was daily and non-daily smoking with seven studies, but the remaining studies were idiosyncratic with two or fewer studies using the same definition.

Heterogeneity in the terminology used for light or non-daily smoking is not a novel observation. Husten (2009) highlighted the same problem, but at this point in time, relatively little was known about non-daily smoking (Fagan and Rigotti 2009). The novel observation here is the even larger degree of heterogeneity when daily smokers are taken into consideration. Daily smokers were the stereotypical image of a smoker and the majority of research considered all smokers to being on the path to becoming daily smokers (Shiffman 2009). Non-daily smoking was just a phase all smokers pass through. However, despite dominating the field, daily smokers could not be consistently defined either. The inconsistencies included subtle differences across 
studies such as having a FTCD score of three or more in one study, and more than three in another study.

The problem of using different criteria was not avoided across the studies presented in this thesis either. Study One was designed before the systematic review was complete and the labels and definitions were based on previous research in attentional bias. Once the benefits of splitting smokers using daily and non-daily smoking habits was acknowledged, Studies Two and Three were consistent with this method. Daily and non-daily smoking was the most common criterion highlighted in the systematic review, and it is the most stable behaviour exhibited by smokers, being frequently used in large population studies (Husten 2009). Unfortunately, daily and nondaily smoking habits were not recorded in Study One and the groupings could not be amended.

Daily and non-daily smokers appears to be the most valid approach for creating smoking groups, but it is unlikely one definitive approach could be lobbied, meaning there are two potential solutions that could improve future research. The first is to test how robust the conclusions are to the criteria used. This was utilised in this thesis when conclusive findings were presented. Study Two was designed to compare daily and non-daily smokers, but the robustness of the conclusions were tested under different criteria. Regardless of whether dichotomies using daily versus nondaily smoking, FTCD scores, or cigarettes per day were used, the results consistently showed there was no significant effect. This has been used effectively in previous research, such as Vollstädt-Klein et al. (2011) and Sweitzer et al. (2008) who used three and two ways of creating smoking groups respectively. Testing the robustness of conclusions under different criteria offers a pragmatic solution where researchers have a favoured approach to defining smoking groups.

The second solution is researchers could avoid dichotomising variables altogether and focus on a correlational approach to continuous data. Several studies in the systematic review used a median split to create smoking groups, but in small samples this will be highly data dependent, creating a new cutoff in each study (MacCallum et al. 2002; Altman and Royston 2006). This explains the subtle differences like the FTCD score example presented above where one study uses three or more and another uses more than three. Studies using a continuous measure were excluded in this systematic 
review as research would often use a token measure of nicotine dependence to explore a correlation with the outcome of interest, but these studies would often be restricted to daily smokers with high inclusion criteria such as in Goudriaan et al. (2010). This meant any studies using a correlational approach were excluded by design, but this was to ensure the studies purposely included non-daily smokers.

\subsection{Attentional bias in daily and non-daily smokers}

The bulk of the thesis investigated attentional bias in daily and non-daily smokers. The first core component of the iRISA model is there should be increased salience to drug-related cues, meaning smokers value the cues and their attention gravitates towards them (Zilverstand et al. 2018). Traditionally, attentional bias was studied in a trait-like manner where different groups were assumed to possess a set level of attentional bias towards drug-related cues. This produced conflicting research with some studies showing that attentional bias towards smoking cues was greater in lighter smokers than heavier smokers (Bradley et al. 2003; Hogarth et al. 2003; Mogg et al. 2005). On the other hand, further research found that heavier smokers displayed greater attentional bias than lighter smokers (Zack et al. 2001; Chanon et al. 2010; Vollstädt-Klein et al. 2011). Study One compared non-smokers, light smokers, and heavier smokers using sample sizes based on previous studies. However, there was no significant difference in response times between the smoking groups and different trial types. This meant smoking cues were not attracting attention and allowing smokers to respond faster. A limitation of this study was the sample size - despite being consistent with previous research - was too small to detect effect sizes of theoretical interest. In response, Study Two was designed to replicate Study One with a larger sample and constrained design.

Study Two was conducted online to gather a large enough sample of daily and non-daily smokers. Despite a sample size including four times as many smokers as Study One, there was still no significant difference in attentional bias towards smoking cues in daily and non-daily smokers. The strength of this study was the sample size was carefully calculated to be informative regardless of the result, and there was evidence in favour of the null using Bayesian analyses. Furthermore, when the results 
of Study One and Two were combined in a mini meta-analysis, there was a negligible, non-significant effect between the two smoking groups. This suggested the effect size was at best much smaller than previous research had assumed. With a sample size approximately three times the size of the next largest study, there was no discernible difference in attentional bias between daily and non-daily smokers.

The combined results of the studies can be viewed in light of the contemporary theory of attentional bias (Field et al. 2016). Rather than view attentional bias as a stable trait-level construct that some types of drug users possess, it is more likely a state-level construct that fluctuates in line with how drug cues are being evaluated. This could be positive evaluations encouraging drug use, or negative evaluations discouraging drug use. Investigating how attentional bias fluctuates was not within the scope of these studies, meaning direct support for this theory had to be provided by other research. Begh et al. (2016) found that measuring attentional bias in the lab as a trait-level construct did not predict subsequent smoking behaviour, but short-term craving and evaluation of drug-cues through ecological momentary assessment did. This suggests that moment to moment evaluations of drug cues may be more informative for drug use than one measurement of attentional bias in a lab environment. What the two studies in this thesis can conclude is trait-level differences between smoking groups are at best much smaller than previously reported.

With 166 participants, Study Two was powered to detect effect sizes almost half as small as the next largest study (Vollstädt-Klein et al. 2011). The observation of previous research reporting significant differences in attentional bias between lighter and heavier smokers can be viewed in line with inflated effect sizes in smaller samples (Etz and Vandekerkhove 2016). If we assume attentional bias fluctuates with the evaluation of smoking cues and participants arrive in their own state, then some studies could observe higher attentional bias in daily smokers and others could observe it in non-daily smokers, purely by chance. However, in larger samples, if there is no manipulation affecting how smoking cues are being evaluated, the differences in attentional bias could cancel each other out and converge towards zero. In order to investigate this, future research should use a large sample of daily and non-daily smokers, while also exploring state-level differences to see if attentional bias changes with the evaluation of smoking cues. 
One of the secondary aims of Studies One and Two was to report the psychometric properties of the visual probe task. The reliability and validity of questionnaires is ubiquitously reported, but cognitive tasks often get a free pass (Hedge et al. 2017; Parsons et al. 2019). Previous research has questioned the internal consistency of the visual probe task, with studies consistently showing that response-time measures are less than adequate (Schmukle 2005; Ataya et al. 2012; Waechter et al. 2014). Christiansen et al. (2015a) reported that the internal consistency of response-time and eyetracking measures could be improved using individualised stimuli. However, more recent attempts showed that this could not be replicated (Jones et al. 2018a). Study One aimed to explore this by using individualised smoking stimuli, providing some participants with images of roll-up cigarettes and others with manufactured cigarettes depending on their preferences. The internal consistency estimate was still less than moderate. A caveat of this study was participants only completed one version, meaning the conclusion was limited to the internal consistency being less than adequate in general rather than comparing two versions using generalised and individualised stimuli like Christiansen et al. (2015a). The poor internal consistency of the task was also demonstrated in Study Two, where a larger sample of smokers completed the visual probe task, but this time using only one version across all participants. Collectively, this shows converging evidence that the visual probe task has inadequate levels of internal consistency.

Low levels of internal consistency may not be an issue for research that focuses on comparing groups or conditions, but it is a problem if unreliable tasks are used for investigating individual differences. Cognitive tasks are designed to exacerbate group differences (Hedge et al. 2017), but this property also means they cannot be ranked and provide consistent measurements for each participant. Low internal consistency in the visual probe task means it is not reliably measuring a single construct across the trials. This has implications for research that aims to use the visual probe task for measuring the effectiveness of an intervention. For example, cognitive bias modification is a large area of research that aims to train attentional bias away from drug-related cues, with the assumption that there is a causal mechanism for attentional bias and drug use. Therefore, if attentional bias can be decreased, then drug use can decrease or become less likely. A narrative review of attentional bias modification studies concluded 
that there was no convincing evidence that it could decrease alcohol or cigarette consumption (Christiansen, Schoenmakers, and Field 2015b). This was also supported by a meta-analysis of 25 studies as there was a very small non-significant effect of cognitive bias modification on attentional bias (Cristea, Kok, and Cuijpers 2016). These studies typically measure attentional bias using a visual probe task, perform a training routine, then measure attentional bias again. If the visual probe task has inadequate internal consistency, it is not reliably measuring a single construct, and a change from one point in time to another could be due to the instability of the measure, rather than a real difference in attentional bias. Based on previous studies (Schmukle 2005; Ataya et al. 2012; Waechter et al. 2014; Jones et al. 2018) and the estimates in Studies One and Two, recording a response-time measure of attentional bias with the visual probe task should not be used for individual differences research as the internal consistency is simply inadequate.

\subsection{Internal consistency of ERP measures of cognitive control}

The primary aim of Study Three was to investigate ERP measures of inhibitory control and error processing in daily and non-daily smokers. The systematic review in Chapter Two highlighted that there were no previous studies that evaluated inhibitory control using a speeded response-time task in daily and non-daily smokers, but one study explored error processing (Rass et al. 2014). The results of Study Three were expected to follow a dose-response relationship with smaller ERP amplitudes in daily smokers than non-daily smokers, and smaller amplitude in non-daily smokers than non-smokers. However, there were no significant effects between the smoking groups across any ERP component associated with inhibitory control and error processing. The main caveat was the sample size of each smoking group was much smaller than what was outlined in the pre-registration protocol. Data collection was slower than expected due to a lack of funding for participants, and the full sample size could not be recruited due to time running out on the PhD programme. This meant the study would only have been able to detect implausibly large effect sizes for neuroscience research. In order to test the substantive hypotheses of Study Three, future research would need to be conducted and ensure the full sample size is recruited. 
The secondary aim of Study Three produced more positive findings. Franken and van der Wetering (2015) was one of the most influential articles in the development of this thesis and they highlighted how it is important to establish the psychometric properties of neurocognitive measures. Study Three showed that ERP components associated with error processing and inhibitory control have moderate to high internal consistency. These estimates support previous work evaluating the psychometric properties of psychophysiology measures. The N2, P3, ERN, and Pe have all been shown to have moderate to high internal consistency (Olvet and Hajcak 2009; Cassidy et al. 2012; Clayson and Larson 2013; Rietdijk et al. 2014). Although test-retest reliability was not explored in Study Three, previous research has demonstrated the four ERP components are moderately stable across measurements from two weeks up to 2.5 years (e.g., Larson et al. 2010; Weinberg and Hajcak 2011). In contrast to the psychometric properties of the visual probe task, the ERP components measured in Study Three may be suitable for individual differences research. Hedge et al. (2017) stated that high internal consistency is critical for correlational research, as the measures should have the ability to consistently distinguish or rank different participants. Therefore, the next step would be to explore how these measures can be used to improve addiction outcomes, either being able to predict cessation success or guide new treatments.

Examining these opportunities in turn, a small number of studies have explored how well ERP components can predict smoking cessation outcomes. Luijten et al. (2016) found that smaller P3 amplitude on the Go/NoGo task was associated with an increased risk of relapse after 12 weeks. However, the N2, ERN, and Pe were not significant predictors. This appears to be the only study investigating ERP components associated with error processing and inhibitory control in smoking cessation outcomes. Given the modest sample size of relapsers and non-relapsers in Luijten et al. (2016), more research is needed to investigate whether other ERP components associated with inhibitory control and error processing can predict relapse rates.

There has been promising research using ERP components of cue reactivity as a predictor of relapse. Versace et al. (2012) showed that a cluster of smokers were less likely to abstain smoking at 24 weeks if they had a lower late positive potential amplitude - an ERP component associated with cue reactivity - towards smoking cues than 
pleasant cues. Placing participants in clusters was based on group-level data that was largely descriptive, preventing individual smokers from being identified as at risk of relapse. The data from Versace et al. (2012) was used to develop a classification algorithm to investigate whether individual smokers higher in late positive potential amplitude towards smoking cues would be less likely to quit smoking (Frank et al. 2019). Smokers classified as at risk were 2.5 times more likely to relapse within 12 months, but there was not a significant decrease due to the small number of successful abstainers. This means that, while promising, the evidence available for individual smokers being identified as at risk of relapse is tentative. More research is needed with a larger sample of smokers who are successfully abstinent at follow-up. It would be interesting to see how this applied to ERP components associated with error processing and inhibitory control as Frank et al. (2019) only focused on components associated with cue reactivity. In summary, there are promising lines of research evaluating how ERP components can be used to predict cessation success. Given the modest sample sizes, further research is necessary to identify if these patterns persist.

The second opportunity for applying neurocognitive measures is guiding new treatments. Given that deficits in inhibitory control have been observed in smokers and associated with an increased risk of relapse, training regimes have been created to manipulate inhibitory control. Similar to cognitive bias modification, inhibitory control training is an intervention designed to improve inhibitory control through participants practicing to inhibit response while being paired with drug-related cues. There is a more substantial body of evidence applying it to alcohol and eating behaviour. Jones et al. (2016) demonstrated that a single session of inhibitory control training led to a small reduction in alcohol or food consumption in a meta-analysis of 18 studies. Allom, Mullan, and Hagger (2016) also found a small reduction in alcohol or food consumption in their meta-analysis of 19 studies. One potential issue is the length of the anticipated effect. Jones et al. (2016) focused on a single session of inhibitory control training and Allom et al. (2016) found that the effect was smaller when measured at a later point in time in comparison to immediately. In addition, these studies are typically cross-sectional and lack adequate controls. The most methodologically sound evidence can be found in a randomised controlled trial where different internetbased inhibitory control training tasks were compared to an active control (Jones et al. 
2018b). All participants lowered their alcohol consumption, but there was no meaningful difference between groups supported by Bayesian analyses. This suggests that inhibitory control training was no better than an active control, with all groups lowering their consumption. This suggests a general effect of study enrolment encouraging people to reduce their consumption levels rather than the interventions. Together these results suggest that inhibitory control training may produce a short-term small reduction in alcohol or food consumption, but when evaluated in a more stringent randomised controlled trial, it may be no better than a control.

The evidence available for the effect of inhibitory control training on smoking behaviour is more sparse and has less promising findings. Implementing a singlesession of inhibitory control training on daily smokers did not reduce cigarette use in comparison to a control group (Adams et al. 2017). This was also the case in a randomised controlled trial implementing internet-based training over a two-week period. All smokers decreased their cigarette use, but there was no significant difference between the training and control group (Bos et al. 2019). This suggests that similar to Jones et al. (2018b), there may be a general effect of study enrolment decreasing cigarette consumption, but no meaningful effect attributable to inhibitory control training. Conversely, inhibitory control training may be able to devalue smoking cues. Scholten et al. (2019) found that smokers and non-smokers would provide more negative evaluations of smoking cues after a single-session of inhibitory control training. Although devaluing cigarette cues may be related to reduced cigarette consumption, they did not directly measure cigarette consumption nor did they include a control group. Together, there is less evidence that inhibitory control training can decrease cigarette consumption, although it may lead to more negative evaluations of cigarette cues.

Considering both opportunities for how neurocognitive measures can be be applied, either in predicting the risk of relapse or being used as an intervention, there is currently more evidence supporting their use in predicting the risk of relapse. The P3 component in inhibitory control (Luijten et al. 2016) and the late positive potential component in cue reactivity (Versace et al. 2012) could predict the risk of relapse at a group-level. There were also promising findings suggesting the late positive potential could be used at an individual level to predict the risk of relapse (Frank et al. 2019), 
but this finding was more tentative. Smoking research has received less attention than other areas including eating behaviour and alcohol consumption. Further research in larger samples could lead to promising insights into how these neurocognitive mechanism can be used to transition from the addiction lab to improving outcomes in the addiction clinic.

\subsection{Limitations}

The limitations specific to each study presented in this thesis were outlined in their respective chapters. The following limitations apply across all the studies, and in most cases to addictive behaviour research in general. In Study One and Three, there was a problem of low statistical power. This was largely due to the thesis topic where the minimum requirement was separate groups of daily and non-daily smokers. Study Two managed to overcome this limitation as it could be conducted online. This meant the study provided more flexibility in recruiting participants and a sufficiently large sample to test the hypotheses could be recruited. However, this limitation was still present in Study Three as it could not be moved online due to the EEG equipment. This meant when fewer participants than expected signed up to the study and there were no resources to recruit further participants, there were few options remaining to collect a sample consistent with the power analysis.

This is not a problem unique to this thesis. Neuroscience research has been criticised for routinely performing studies with low statistical power (Button et al. 2013). In their review of meta-analyses, the studies in each meta-analysis had low power to detect the effect size estimated in that particular meta-analysis. A common retort to the pervasive problem of low statistical power in neuroscience research is studies that use brain imaging methods like EEG and fMRI require more resources for data collection than an online study. While this may be the case for individual research groups, one of the solutions raised by Button et al. (2013) is to work collaboratively and pool resources. This means that individual groups may only be able to collect a limited amount of data, but when data is pooled together from different groups, there will be a large enough sample to have adequate statistical power to test substantive predictions. This approach has recently grown in psychology with the creation of the 
Psychological Science Accelerator (Moshontz et al. 2018), a network of labs across the world that pools resources to achieve a more representative and larger sample than would be possible with the means of a single research group. This approach has also been applied to undergraduate students with the Collaborative Replications and Education Project (CREP; Wagge et al. 2019) and large replication attempts like the Many Labs projects (e.g., Ebersole et al. 2016). Although the motivation behind these projects differs, the main similarity is the use of a distributed network of research groups to pool resources and conduct more informative research than would be capable alone. In future, a similar approach would be beneficial for projects like Study Three as it involved specific populations (two smoking sub-groups) and resource intensive data collection. In PhD programmes, each doctoral student could design an element of the study to test their novel contribution, but data collection would be pooled across different research groups to obtain a larger sample. This would have the potential to perform more informative research while still meeting the requirements of doctoral research.

The next limitation applies to the limit of what neurobiological models of addiction can offer in terms of explanation and treatment. The systematic review in Chapter Two was organised using the iRISA model of drug addiction (Goldstein and Volkow 2002; 2011; Zilverstand et al. 2018) which explains addictive behaviour through a syndrome of overactive drive processes and under-active control processes. The model has been hugely influential for research in addictive behaviour and builds on the work of animal models of addiction, but models like iRISA have come under vocal criticism. Field and Kersbergen (2019) argued that animal models of addiction and theories built on them have been oversold and possess severe limitations. Due to uniquely human qualities characterising addiction such as wanting to stop drug use but being unable to, animal models can only provide a reductionist overview of addictive behaviour that do not take these human elements into account. Therefore, the neurobiological basis of addiction should only be considered one factor in a collection of influences (Field, Heather, and Wiers 2019). For example, the iRISA model considers drug use to lead to permanent deficits in regions such as the prefrontal cortex. If there is a causal relationship between these deficits and addictive behaviour, it does not explain how some people with drug use problems can permanently cease their drug use without 
the use of treatment (Field et al. 2019). Consistent with many psychological phenomena, it is almost certainly more complicated than being explained by one factor. It may be a better approach to consider a network of different influences that includes biological factors, but in addition to wider socioeconomic problems that influence and perpetuate problematic drug use (Borsboom, Cramer, and Kalis 2018). The studies presented in this thesis only focused on neurocognitive mechanisms, with these individual factors only contributing to the puzzle of addictive behaviour, meaning they are not going to provide the full explanation. This is not meant to downplay the contribution of neurocognitive mechanisms to addictive behaviour, but it is important to outline what remit the findings in this thesis contribute to.

Within the scope of neurocognitive mechanisms, there are also more complicated factors relating to addictive behaviour. In West and Brown's (2013) PRIME theory of addiction, responses, impulses, and inhibitions only take up the first two levels of addiction, with the concepts in this thesis (attentional bias, error processing, and inhibitory control) relating to these two levels. There are more complicated factors which encapsulates plans, motives, and evaluations. Plans are the highest level and require deliberation to determine whether a behaviour should or should not be executed. Higher levels can moderate the effects of lower levels, for example, there could be a delayed plan where a particular behaviour is not appropriate at one moment in time, but it will be in future. One demonstration of how these levels may interact is the effect of restraint on inhibitory control training (Jones et al. 2016). In their meta-analysis, there was a moderate reduction in food consumption when participants reported exercising restraint, but there was a small non-significant effect when they reported low restraint. Although there is the caveat that this research applies to eating behaviour and not smoking, there may be an interaction between different factors relating to addictive behaviour where simple mechanisms are expressed differently depending on more complicated factors relating to planning and deliberation. The studies presented in this thesis only focused on behaviours relating to the first two levels of the PRIME theory (West and Brown 2013), and more complicated factors were not taken into consideration. In future research, factors such as attentional bias and inhibitory control may be key as building blocks, but investigating how higher level neurocognitive factors such as plans and motives moderate their expression will 
be important for understanding addictive behaviour.

The studies presented in this thesis and all but one study in the systematic review were cross-sectional. This means they could not address whether neurocognitive deficits are a cause or consequence of drug use. Several prospective studies have demonstrated that deficits in neurocognitive functioning can precede the onset of drug use. Harakeh et al. (2012) tracked adolescents from age 11 to age 16. Lower response inhibition at age 11 - as measured through a shifting-set task - was associated with a greater risk of starting to smoke by age 16. Poor sustained attention was also associated with being a daily smoker at age 16. Ersche et al. (2012) compared healthy non-smoking controls with stimulant dependent participants and a non-dependent biological sibling. Both the stimulant dependent participants and their biological siblings had significantly worse inhibitory control than healthy controls. Shared deficits in inhibitory control despite differences in stimulant dependence suggests that there may be a biological predisposition to problematic drug use. The caveat of applying this study here is smoking rates were higher in the sibling pairs, but the sample focused on poly-drug use, rather than isolating the effect of smoking. There is also evidence that reduced ERN amplitude predicts smoking initiation by age 18 (Anokhin and Golosheykin 2015). Adolescents were followed in a longitudinal study from the age of 12, and had repeated ERP recordings at ages 14 and 16. There was not a significant effect of ERN amplitude at age 12, but smaller amplitude at ages 14 and 16 were associated with greater risk of smoking at age 18. Collectively, these studies suggest that pre-existing deficits in neurocognitive performance may be a risk factor for starting to smoke. The prospective nature of the studies were important for demonstrating that the risk factors preceded drug use. As the studies presented in this thesis were cross-sectional, it would not have been possible to comment on the development of mechanisms like inhibitory control and error processing, only how they appeared at one point in time.

These prospective studies can also be viewed in line with the previous limitation of addiction being the results of myriad factors as despite controlling for gender, socioeconomic status, and baseline performance, the predictors in Harakeh et al. (2012) explained less than $5 \%$ of the variance in smoking behaviour. Wellman et al. (2016) performed a comprehensive review of 53 prospective studies investigating the onset 
of smoking behaviour in later adolescence. Among the studies, there were 98 unique predictors of smoking onset, demonstrating the multitude of risk factors. Some of the most consistent factors across studies were lower socio-economic status, parental and peer smoking, lower self-esteem, and more prevalent tobacco marketing. Deficits in neurocognitive mechanism may contribute to the onset of smoking behaviour, but they are only a small piece of the puzzle. Additional factors are required to explain the onset and development of complex patterns of behaviour such as addiction.

There is also evidence that deficits in neurocognitive functioning can be reduced after stopping smoking. Fried et al. (2006) was the only prospective study included in the systematic review presented in Chapter Two. They compared light and heavy smokers to non-smokers and former smokers in their general neurocognitive ability between the ages of 9-12 and in later adolescence. Smoking was associated with poorer performance on measures of vocabulary, verbal IQ, and verbal comprehension. However, the deficits were much smaller in former smokers who had quit smoking for six months or more. There appears to be few studies evaluating how well neurocognitive functioning can return in smokers beyond adolescence. A review of longitudinal studies evaluating the recovery of neurocognitive functioning found that different drug users including alcohol, cocaine, and methamphetamine could partially recover after long-term abstinence in comparison to healthy controls (Schulte et al. 2014). This should be interpreted with the caveat of none of the studies included a group of smokers, meaning the results suggest that neurocognitive functioning can partially recover, but studies on smokers would need to be included to be directly applicable. These studies suggest that neurocognitive functioning may also be malleable across the lifespan. Long-term cessation may lead to (at least partially) reversing deficits to neurocognitive functioning.

The studies included in this thesis were cross-sectional, meaning that they could not provide inferences about how deficits in neurocognitive mechanisms could precede drug-use, or change along the life-span as drug use continues or stops. Prospective studies have shown that deficits in neurocognitive mechanisms early in adolescence can precede drug use and increase the likelihood of smoking later in life. Longitudinal studies have also shown neurocognitive functioning can return with the cessation of drug-use, with former smokers similar to non-smokers in adolescence (Fried 
et al. 2006), and adult drug users at least partially recovering (Schulte et al. 2014). Therefore, the studies presented in this thesis only demonstrate a snapshot of neurocognitive mechanisms at one point in time, where they may fluctuate across the lifecycle of drug use.

Most of these studies include a single smoking group who are nicotine dependent. It would be interesting to investigate how neurocognitive functioning changes in daily and non-daily smokers. Fried et al. (2006) included a group of light and heavy smokers in their longitudinal study on general neurocognitive functioning, but they did not report comparisons between the two smoking groups, only one or the other in comparison to non-smokers. Robertson et al. (2016) reported a longitudinal study following smokers over a 17 year period. There was some fluctuation in whether people were non-daily, low-rate daily, and high-rate daily at age 21 and then at age 38. Some smokers remained in the same groups, with the majority of non-daily smokers continuing to be non-daily smokers or becoming non-smokers. High-rate daily smokers were split between transitioning to non-smokers or non-daily smokers, or continuing as high-rate smokers. This suggests that there is some long-term fluctuation in the smoker's habits, and it would be interesting to see how neurocognitive functioning changes as they transition between daily and non-daily smokers. There does not appear to be any research that has explored this.

\subsection{Future research}

Areas for future research have been identified in response to the general limitations, but there are some specific opportunities for future research that follow on from the studies presented in this thesis. Studies One and Two found that trait-level differences in attentional bias between daily and non-daily smokers are at best much smaller than previously reported. This is consistent with a contemporary theory of attentional bias where it may be better explored as a state-level construct that changes with the moment to moment evaluation of drug cues (Field et al. 2016). An alternative approach would be to investigate what factors can influence the expression of attentional bias. One particularly important factor for daily and non-daily smokers is the influence of alcohol. There is a close relationship with smoking and drinking alcohol, and this 
relationship appears to be particularly pronounced in non-daily smokers (Tong et al. 2006; Witkiewitz et al. 2012; Hoeppner et al. 2014; McGrath et al. 2015). One study has shown that attentional bias towards smoking cues is greater after drinking alcohol in daily smokers (Field et al. 2005), but this has not been extended to compare daily and non-daily smokers. Taking their smoking motives into consideration where there appears to be a stronger relationship between alcohol and smoking, non-daily smokers may show greater attentional bias towards smoking cues than daily smokers. Given there is only one known study on the effect of alcohol on attentional bias in smokers, this could be an interesting extension.

The research presented in this thesis focused on attentional bias and cognitive control. While they were investigated separately here, there is a direct link between the two concepts (Marhe et al. 2014). The iRISA model (Goldstein and Volkow 2002; 2011; Zilverstand et al. 2018) predicts there is a combination of poor cognitive control and excessive salience to drug cues underlying addiction. This means that it is particularly difficult to inhibit drug use when presented with salient drug cues. To more comprehensively test the predictions of the iRISA model, the two mechanisms could be studied together in future research. Some types of smoker may find it more difficult to inhibit their responses when distracted by a smoking cue in comparison to a neutral cue that is less salient.

There have been mixed findings in the small number of studies that have attempted to investigate the effects of smoking cues on cognitive control. Luijten et al. (2011a) used an adapted version of the Go/NoGo task where Go and NoGo cues were coloured borders around smoking or neutral images. Despite smokers having lower N2 amplitude than non-smokers on NoGo trials requiring inhibition, there was no significant interaction with the type of image used. Smokers and non-smokers responded to each image type similarly. Luijten et al. (2011b) investigated the effect of smoking and neutral images on an adapted version of the Eriksen Flanker task for error processing, but there was not a significant interaction with response time measures. There were too few error trials in each image condition to investigate the effects on ERP components. Detandt et al. (2017) used an adapted version of the Go/NoGo task with the smoking and neutral background images being presented for whole blocks, rather than changing on each trial. In contrast to Luijten et al. (2011a; 2011b), smokers made fewer 
mistakes and had a higher P3 amplitude than non-smokers when a smoking background was used. This suggests that it may help smokers to focus in the region where the stimuli are presented, as smoking images attract the smoker's attention, and allow them to accurately respond to the Go and NoGo cues.

There are two components here that would justify future research. First, there is only a small number of studies reporting mixed findings on the effect of a smoking background on smokers' cognitive control ability. Second, these studies only included one smoking group, who smoked at a daily level. None of the studies included nondaily smokers in their sample. Therefore, future research could replicate the effect of higher P3 amplitude in smokers when a smoking background is used (Dedandt et al. 2017), and extend it to include daily and non-daily smokers.

\subsection{Conclusions}

The aim of this thesis was to create a focused profile of neurocognitive mechanisms associated with addictive behaviour in daily and non-daily smokers. There is a large body of research investigating these mechanisms by comparing nicotine dependent smokers to non-smokers, but there is less research focusing on different types of smokers, namely daily and non-daily smokers. Research focusing on smokers as a single group has generally found more severe deficits are associated with greater dependence and worse cessation outcomes, but these studies typically exclude non-daily smokers. It was important to investigate daily and non-daily smokers as despite displaying fundamentally different smoking habits, they demonstrate similar long-term relapse rates. This means if these neurocognitive mechanisms are associated with the maintenance of drug use, then exploring the differences between daily and non-daily smokers would be an important area of research to understand factors associated with their smoking behaviour.

This thesis provided three main contributions to understanding neurocognitive mechanisms in daily and non-daily smokers. First, the systematic review was the first to explore these mechanisms in daily and non-daily smokers. Previous reviews had explored neurocognitive mechanisms associated with drug use and different smoking groups in isolation, but this systematic review was the first to combine the topics. 
One of the key findings from this review was the observation that there is a problematic level of heterogeneity in the labels and definitions used to investigate different smoking groups. There were almost as many unique ways of labelling and defining lighter and heavier smokers as the number of studies included in the review. Future research should think carefully about how different smoking groups are created, with potential solutions including using more valid approaches like differentiating daily and non-daily smokers, exploring how robust conclusions are to different ways of categorising smokers, or using correlational approaches to treat nicotine dependence as a continuum.

Second, Studies One and Two explored attentional bias to smoking cues with Study Two using the largest known sample size to date. Using a visual probe task, there was no discernible difference in attentional bias between daily and non-daily smokers. This can be interpreted as stable trait-level differences in attentional bias are unlikely and previous research may have reported false positives where attentional bias fluctuated in the direction of daily or non-daily smokers. However, when a larger sample size was used in Study Two, these fluctuations may have cancelled out and there was a very small effect between daily and non-daily smokers. Future research should focus on exploring state-level fluctuations in attentional bias rather than conceptualise attentional bias as a stable trait-level construct. As a secondary aim, Studies One and Two supported previous research highlighting the poor psychometric properties of the visual probe task. Therefore, if future research explores how attentional bias fluctuates across multiple time points, more internally consistent measures are required such as direct measurements of eye movements.

Finally, in contrast to the psychometric properties of the visual probe task, the internal consistency of ERP measures of inhibitory control and error processing was moderate to high. This means they are best placed for predicting cessation outcomes or being directly targeted in interventions due to their stability within- and acrosssessions. This supported previous research which demonstrated the good psychometric properties of ERP measures of inhibitory control and error processing. However, the main aim of Study Three was to investigate the difference in inhibitory control and error processing in daily smokers, non-daily smokers, and non-smokers. There were no significant effects of interest, but this was more than likely due to the sample 
size being too small to make meaningful conclusions. Difficulties with data collection meant the pre-registered sampling plan was not fulfilled and the results were inconclusive. This means the main contribution of Study Three was a secondary aim about the moderate to high psychometric properties of the measures used.

In conclusion, this thesis presented a focused profile of measures relating to drive and control neurocognitive mechanisms in daily and non-daily smokers. The rationale behind this aim was if neurocognitive mechanisms were important to the maintenance of addictive behaviour, they might be able to explain how daily and non-daily smokers can display fundamentally different smoking habits, but similar long-term cessation rates. In Studies Two and Three where smokers were categorised as daily and nondaily smokers, their characteristics were compatible with previous research as nondaily smokers had consistently lower levels of nicotine dependence across different measures. However, the studies presented in this thesis were less positive about the utility of drive and control components, particularly attentional bias. This means if these neurocognitive mechanisms contribute to addictive behaviour, future research will have to investigate their role using alternative designs. For attentional bias, it will be important to focus on differences across states, and for cognitive control, it will be important to include a sufficiently large sample size. As it stands with the methods used in this thesis, there were no meaningful differences between daily and non-daily smokers that could explain their shared difficulty in quitting smoking long-term. 


\section{Chapter 6}

\section{References}

Adams, S., Mokrysz, C., Attwood, A.S., and Munafó, M.R. (2017) 'Resisting the Urge to Smoke: Inhibitory Control Training in Cigarette Smokers'. Royal Society Open Science 4 (8), 1-7

Advokat, C. D., Comaty, J. E. and Julien, R. M. (2014) Julien's Primer of Drug Action. New York: Worth Publishers

Action on Smoking and Health (2020) Smoking Statistics [online] available from https : //ash.org.uk/wp-content/uploads/2019/10/SmokingStatistics.pdf [24April2020]

Allom, V., Mullan, B., and Hagger, M. (2016) 'Does Inhibitory Control Training Improve Health Behaviour? A Meta-Analysis'. Health Psychology Review 10 (2), 168-186

Altman, D.G. and Royston, P. (2006) 'The Cost of Dichotomising Continuous Variables'. British Medical Journal 332 (7549), 1080

Anokhin, A.P. and Golosheykin, S. (2015) 'Neural Correlates of Error Monitoring in Adolescents Prospectively Predict Initiation of Tobacco Use'. Developmental Cognitive Neuroscience 16, 166-173

Anokhin, A.P., Vedeniapin, A.B., Sirevaag, E.J., Bauer, L.O., O'Connor, S.J., Kuperman, S., Porjesz, B., Reich, T., Begleiter, H., Polich, J., and Rohrbaugh, J.W. (2000) 'The P300 Brain Potential Is Reduced in Smokers'. Psychopharmacology 149 (4), 409-413 
Anwyl-Irvine, A., Massonnié, J., Flitton, A., Kirkham, N., and Evershed, J. (2019) 'Gorilla in Our Midst: An Online Behavioral Experiment Builder'. Behavior Research Methods $52,388-407$

Ashe, M. L., and Wilson, S. J. (2020). Very Light Daily Smoking in Emerging Adults: Relationships Between Dependence and Lapse. Preprint retrieved from https://doi . org/10.31234/osf.io/z2n9v

Ataya, A.F., Adams, S., Mullings, E., Cooper, R.M., Attwood, A.S., and Munafó, M.R. (2012) 'Internal Reliability of Measures of Substance-Related Cognitive Bias'. Drug and Alcohol Dependence 121 (1), 148-151

Bakeman, R. (2005) 'Recommended Effect Size Statistics for Repeated Measures Designs'. Behavior Research Methods 37 (3), 379-384

Bakker, M., Hartgerink, C.H.J., Wicherts, J.M., and van der Maas, H.L.J. (2016) 'Researchers' Intuitions About Power in Psychological Research'. Psychological Science 27 (8), 1069-1077

Baldwin, S.A. (2017) 'Improving the Rigor of Psychophysiology Research'. International Journal of Psychophysiology 111, 5-16

Balfour, D.J.K. (2004) 'The Neurobiology of Tobacco Dependence: A Preclinical Perspective on the Role of the Dopamine Projections to the Nucleus Accumbens'. Nicotine $\mathcal{E}$ Tobacco Research 6 (6), 899-912

Balfour, D.J.K. (2008) 'The Psychobiology of Nicotine Dependence'. European Respiratory Review 17 (110), 172-181

Baschnagel, J.S. (2013) 'Using Mobile Eye-Tracking to Assess Attention to Smoking Cues in a Naturalized Environment'. Addictive Behaviors 38 (12), 2837-2840 
Beard, E., West, R., Michie, S., and Brown, J. (2020) 'Association of Prevalence of Electronic Cigarette Use with Smoking Cessation and Cigarette Consumption in England: A Time?Series Analysis between 2006 and 2017'. Addiction 115 (5), 961 ?974

Begh, R., Smith, M., Ferguson, S., Shiffman, S., Munafó, M., and Aveyard, P. (2016) 'Association between Smoking-Related Attentional Bias and Craving Measured in the Clinic and in the Natural Environment'. Psychology of Addictive Behaviors 30 (8), 868-875

Benowitz, N. (2010) 'Nicotine Addiction'. The New England Journal of Medicine 362 (24), 2295-2303

Berkman, E.T., Falk, E.B., and Lieberman, M.D. (2011) 'In the Trenches of Real-World Self-Control: Neural Correlates of Breaking the Link between Craving and Smoking'. Psychological Science 22 (4), 498-506

Billieux, J., Gay, P., Rochat, L., Khazaal, Y., Zullino, D., and Van der Linden, M. (2010) 'Lack of Inhibitory Control Predicts Cigarette Smoking Dependence: Evidence from a Non-Deprived Sample of Light to Moderate Smokers'. Drug and Alcohol Dependence $112(1-2), 164-167$

Birge, M., Duffy, S., Miler, J.A., and Hajek, P. (2018) 'What Proportion of People Who Try One Cigarette Become Daily Smokers? A Meta-Analysis of Representative Surveys'. Nicotine $\mathcal{E}$ Tobacco Research 20 (12), 1427-1433

Birkás, B., Dzhelyova, M., Lábadi, B., Bereczkei, T., and Perrett, D.I. (2014) 'CrossCultural Perception of Trustworthiness: The Effect of Ethnicity Features on Evaluation of Faces' Observed Trustworthiness across Four Samples'. Personality and Individual Differences 69, 56-61

Bogdanovica, I., Godfrey, F., McNeill, A., and Britton, J. (2011) 'Smoking Prevalence in the European Union: A Comparison of National and Transnational Prevalence Survey 
Methods and Results'. Tobacco Control 20 (1), 1-9

Borenstein, M., Hedges, L.V., Higgins, J.P.T., and Rothstein, H.R. (2010) 'A Basic Introduction to Fixed-Effect and Random-Effects Models for Meta-Analysis'. Research Synthesis Methods 1 (2), 97-111

Borenstein, M., Hedges, L. V., Higgins, J. P., and Rothstein, H. R. (2011). Introduction to Meta-analysis. John Wiley \& Sons

Borsboom, D., Cramer, A., and Kalis, A. (2018) 'Brain Disorders? Not Really? Why Network Structures Block Reductionism in Psychopathology Research'. The Behavioral and Brain Sciences 24, 1-54

Bos, J., Staiger, P.K., Hayden, M.J., Hughes, L.K., Youssef, G., and Lawrence, N.S. (2019) 'A Randomized Controlled Trial of Inhibitory Control Training for Smoking Cessation and Reduction'. Journal of Consulting and Clinical Psychology 87 (9), 831-843

Botvinick, M.M., Cohen, J.D., and Carter, C.S. (2004) 'Conflict Monitoring and Anterior Cingulate Cortex: An Update'. Trends in Cognitive Sciences 8 (12), 539-546

Boudewyn, M.A., Luck, S.J., Farrens, J.L., and Kappenman, E.S. (2017) 'How Many Trials Does It Take to Get a Significant ERP Effect? It Depends'. Psychophysiology 55 (6), $1-16$

Bradley, B.P., Mogg, K., Wright, T., and Field, M. (2003) 'Attentional Bias in Drug Dependence: Vigilance for Cigarette-Related Cues in Smokers'. Psychology of Addictive Behaviors 17 (1), 66-72

Brand, A. and Bradley, M.T. (2012) 'Assessing the Effects of Technical Variance on the Statistical Outcomes of Web Experiments Measuring Response Times'. Social Science Computer Review 30 (3), 350-357 
Breslau, N. and Peterson, E.L. (1996) 'Smoking Cessation in Young Adults: Age at Initiation of Cigarette Smoking and Other Suspected Influences'. American Journal of Public Health 86 (2), 214-221

Brinkmeyer, J., Mobascher, A., Musso, F., Schmitz, M., Wagner, M., Frommann, I., Gründer, G., Spreckelmeyer, K.N., Wienker, T., Díaz-Lacava, A., Holler, D., Dahmen, N., Thuerauf, N., Clepce, M., Kiefer, F., de Millas, W., Gallinat, J., and Winterer, G. (2011) 'P50 Sensory Gating and Smoking in the General Population: P50 Sensory Gating and Smoking'. Addiction Biology 16 (3), 485-498

Brown, A.E., Carpenter, M.J., and Sutfin, E.L. (2011) 'Occasional Smoking in College: Who, What, When and Why?'. Addictive Behaviors 36 (12), 1199-1204

Brown, C.R.H., Forster, S., and Duka, T. (2018) 'Goal-Driven Attentional Capture by Appetitive and Aversive Smoking-Related Cues in Nicotine-Dependent Smokers'. Drug and Alcohol Dependence 190, 209-215

Bühler, M., Vollstädt-Klein, S., Kobiella, A., Budde, H., Reed, L.J., Braus, D.F., Büchel, C., and Smolka, M.N. (2010) 'Nicotine Dependence Is Characterized by Disordered Reward Processing in a Network Driving Motivation'. Biological Psychiatry 67 (8), 745-752

Burle, B., Spieser, L., Roger, C., Casini, L., Hasbroucq, T., and Vidal, F. (2015) 'Spatial and Temporal Resolutions of EEG: Is It Really Black and White? A Scalp Current Density View'. International Journal of Psychophysiology 97 (3), 210-220

Butler, K., Rusted, J., Gard, P., and Jackson, A. (2017) 'Performance Monitoring in Nicotine Dependence: Considering Integration of Recent Reinforcement History'. Pharmacology Biochemistry and Behavior 156, 63-70

Button, K.S., Ioannidis, J.P.A., Mokrysz, C., Nosek, B.A., Flint, J., Robinson, E.S.J., and Munafó, M.R. (2013) 'Power Failure: Why Small Sample Size Undermines the Reliability of Neuroscience'. Nature Reviews Neuroscience 14 (5), 365-376 
Buzzell, G.A., Fedota, J.R., Roberts, D.M., and McDonald, C.G. (2014) 'The N2 ERP Component as an Index of Impaired Cognitive Control in Smokers'. Neuroscience Letters $563,61-65$

Carim-Todd, L., Mitchell, S.H., and Oken, B.S. (2016) 'Impulsivity and Stress Response in Nondependent Smokers (Tobacco Chippers) in Comparison to Heavy Smokers and Nonsmokers'. Nicotine E Tobacco Research 18 (5), 547-556

Carroll, A.J., Sutherland, M.T., Salmeron, B.J., Ross, T.J., and Stein, E.A. (2015) 'Greater Externalizing Personality Traits Predict Less Error-Related Insula and Anterior Cingulate Cortex Activity in Acutely Abstinent Cigarette Smokers'. Addiction Biology 20 (2), 377-389

Cassidy, S.M., Robertson, I.H., and O'Connell, R.G. (2012) 'Retest Reliability of EventRelated Potentials: Evidence from a Variety of Paradigms'. Psychophysiology 49 (5), 659-664

Chaffee, B.W., Watkins, S.L., and Glantz, S.A. (2018) 'Electronic Cigarette Use and Progression From Experimentation to Established Smoking'. Pediatrics 141 (4), 1-6

Chanon, V.W., Sours, C.R., and Boettiger, C.A. (2010) 'Attentional Bias toward Cigarette Cues in Active Smokers'. Psychopharmacology 212 (3), 309-320

Christiansen, P., Mansfield, R., Duckworth, J., Field, M., and Jones, A. (2015a) 'Internal Reliability of the Alcohol-Related Visual Probe Task Is Increased by Utilising Personalised Stimuli and Eye-Tracking'. Drug and Alcohol Dependence 155, 170-174

Christiansen, P., Schoenmakers, T.M., and Field, M. (2015b) 'Less than Meets the Eye: Reappraising the Clinical Relevance of Attentional Bias in Addiction'. Addictive Behaviors $44,43-50$ 
Clayson, P.E., Baldwin, S.A., and Larson, M.J. (2013) 'How Does Noise Affect Amplitude and Latency Measurement of Event-Related Potentials (ERPs)? A Methodological Critique and Simulation Study: ERP Measurement'. Psychophysiology 50 (2), 174-186

Clayson, P.E. and Larson, M.J. (2013) 'Psychometric Properties of Conflict Monitoring and Conflict Adaptation Indices: Response Time and Conflict N2 Event-Related Potentials: Conflict Adaptation Psychometrics'. Psychophysiology 50 (12), 1209-1219

Clayson, P.E. and Miller, G.A. (2017) 'ERP Reliability Analysis (ERA) Toolbox: An Open-Source Toolbox for Analyzing the Reliability of Event-Related Brain Potentials'. International Journal of Psychophysiology 111, 68-79

Clifford, S. and Jerit, J. (2014) 'Is There a Cost to Convenience? An Experimental Comparison of Data Quality in Laboratory and Online Studies'. Journal of Experimental Political Science 1 (2), 120-131

Cochran, J.R., Kydd, R.R., Lee, J.M.J., Walker, N., and Consedine, N.S. (2018) 'Disgust but Not Health Anxiety Graphic Warning Labels Reduce Motivated Attention in Smokers: A Study of P300 and Late Positive Potential Responses'. Nicotine E Tobacco Research 20 (7), 819-826

Coggins, C.R.E., Murrelle, E.L., Carchman, R. A, and Heidbreder, C. (2009) 'Light and Intermittent Cigarette Smokers: A Review (1989-2009)'. Psychopharmacology 207 (3), $343-63$

Conti, A.A., McLean, L., Tolomeo, S., Steele, J.D., and Baldacchino, A. (2019) 'Chronic Tobacco Smoking and Neuropsychological Impairments: A Systematic Review and Meta-Analysis'. Neuroscience \& Biobehavioral Reviews 96, 143-154

Cooper, T. V., Taylor, T., Murray, A., DeBon, M.W., Vander Weg, M.W., Klesges, R.C., and Talcott, G.W. (2010) 'Differences between Intermittent and Light Daily Smokers in 
a Population of U.S. Military Recruits'. Nicotine E Tobacco Research 12 (5), 465-473

Cox, L.S., Tiffany, S.T., and Christen, A.G. (2001) 'Evaluation of the Brief Questionnaire of Smoking Urges (QSU-Brief) in Laboratory and Clinical Settings'. Nicotine $\mathcal{E}$ Tobacco Research 3, 7-16

Cramer, A.O.J., van Ravenzwaaij, D., Matzke, D., Steingroever, H., Wetzels, R., Grasman, R.P.P.P., Waldorp, L.J., and Wagenmakers, E.-J. (2016) 'Hidden Multiplicity in Exploratory Multiway ANOVA: Prevalence and Remedies'. Psychonomic Bulletin $\mathcal{E}$ Review 23 (2), 640-647

Crawford, J.R. and Henry, J.D. (2004) 'The Positive and Negative Affect Schedule (PANAS): Construct Validity, Measurement Properties and Normative Data in a Large Non-Clinical Sample'. The British Journal of Clinical Psychology 43, 245-265

Cristea, I.A., Kok, R.N., and Cuijpers, P. (2016) 'The Effectiveness of Cognitive Bias Modification Interventions for Substance Addictions: A Meta-Analysis'. PLoS ONE 11 (9), $\mathrm{e} 0162226$

Crunelle, C.L., Veltman, D.J., Booij, J., Van Emmerik-van Oortmerssen, K., and Van Den Brink, W. (2012) 'Substrates of Neuropsychological Functioning in Stimulant Dependence: A Review of Functional Neuroimaging Research'. Brain and Behavior 2 (4), 499-523

Crump, M.J.C., McDonnell, J.V., and Gureckis, T.M. (2013) 'Evaluating Amazon's Mechanical Turk as a Tool for Experimental Behavioral Research'. PLoS ONE 8 (3), e57410

Cui, Y., Robinson, J.D., Versace, F., Lam, C.Y., Minnix, J.A., Karam-Hage, M., Dani, J.A., Kosten, T.R., Wetter, D.W., Brown, V.L., and Cinciripini, P.M. (2012) 'Differential Cigarette-Related Startle Cue Reactivity among Light, Moderate, and Heavy Smokers'. Addictive Behaviors 37 (8), 885-889 
Cyders, M.A. and Coskunpinar, A. (2011) 'Measurement of Constructs Using SelfReport and Behavioral Lab Tasks: Is There Overlap in Nomothetic Span and Construct Representation for Impulsivity?'. Clinical Psychology Review 31 (6), 965-982

Darlow, S. and Lobel, M. (2012) 'Smoking Behavior and Motivational Flexibility in Light and Heavy Smokers'. Addictive Behaviors 37 (5), 668-673

de Ruiter, M.B., Oosterlaan, J., Veltman, D.J., van den Brink, W., and Goudriaan, A.E. (2012) 'Similar Hyporesponsiveness of the Dorsomedial Prefrontal Cortex in Problem Gamblers and Heavy Smokers during an Inhibitory Control Task'. Drug and Alcohol Dependence 121 (1-2), 81-89

Detandt, S., Bazan, A., Quertemont, E., and Verbanck, P. (2017) 'Smoking addiction: The shift from head to hands: Approach bias towards smoking-related cues in lowdependent versus dependent smokers'. Journal of Psychopharmacology 31(7), 819-829

Delacre, M., Lakens, D., and Leys, C. (2017) 'Why Psychologists Should by Default Use Welch's T-Test Instead of Student's T-Test'. International Review of Social Psychology $30(1), 92-101$

Dickter, C.L. and Forestell, C.A. (2012) 'Peering through the Smoke: The Effect of Parental Smoking Behavior and Addiction on Daily Smokers' Attentional Bias to Smoking Cues'. Addictive Behaviors 37 (2), 187-192

Dienes, Z., (2008). Understanding psychology as a science: An introduction to scientific and statistical inference. Macmillan International Higher Education.

Dunn, T.J., Baguley, T., and Brunsden, V. (2014) 'From Alpha to Omega: A Practical Solution to the Pervasive Problem of Internal Consistency Estimation'. British Journal of Psychology 105 (3), 399-412

Dutra, T., Formagini, B., Pinto, H., Perales, J., Antonio, F., and Colugnati, B. (2017) 
'Prevalence and Correlates of Light and Non-Daily Smoking in Brazil: Results from a Nationwide Representative Survey'. Drug and Alcohol Dependence 178, 15-19

Ebersole, C.R., Atherton, O.E., Belanger, A.L., Skulborstad, H.M., Allen, J.M., Banks, J.B., Baranski, E., Bernstein, M.J., Bonfiglio, D.B.V., Boucher, L., Brown, E.R., Budiman, N.I., Cairo, A.H., Capaldi, C.A., Chartier, C.R., Chung, J.M., Cicero, D.C., Coleman, J.A., Conway, J.G., Davis, W.E., Devos, T., Fletcher, M.M., German, K., Grahe, J.E., Hermann, A.D., Hicks, J.A., Honeycutt, N., Humphrey, B., Janus, M., Johnson, D.J., Joy-Gaba, J.A., Juzeler, H., Keres, A., Kinney, D., Kirshenbaum, J., Klein, R.A., Lucas, R.E., Lustgraaf, C.J.N., Martin, D., Menon, M., Metzger, M., Moloney, J.M., Morse, P.J., Prislin, R., Razza, T., Re, D.E., Rule, N.O., Sacco, D.F., Sauerberger, K., Shrider, E., Shultz, M., Siemsen, C., Sobocko, K., Weylin Sternglanz, R., Summerville, A., Tskhay, K.O., van Allen, Z., Vaughn, L.A., Walker, R.J., Weinberg, A., Wilson, J.P., Wirth, J.H., Wortman, J., and Nosek, B.A. (2016) 'Many Labs 3: Evaluating Participant Pool Quality across the Academic Semester via Replication'. Journal of Experimental Social Psychology $67,68-82$

Ehrman, R.N., Robbins, S.J., Bromwell, M.A., Lankford, M.E., Monterosso, J.R., and O'Brien, C.P. (2002) 'Comparing Attentional Bias to Smoking Cues in Current Smokers, Former Smokers, and Non-Smokers Using a Dot-Probe Task'. Drug and Alcohol Dependence 67 (2), 185-191

Eriksen, B.A. and Eriksen, C.W. (1974) 'Effects of Noise Letters upon the Identification of a Target Letter in a Nonsearch Task'. Perception \& Psychophysics 16 (1), 143-149

Ersche, K.D., Jones, P.S., Williams, G.B., Turton, A.J., Robbins, T.W., and Bullmore, E.T. (2012) 'Abnormal Brain Structure Implicated in Stimulant Drug Addiction'. Science $335,601-604$

Etz, A. and Vandekerckhove, J. (2016) 'A Bayesian Perspective on the Reproducibility Project: Psychology'. PLoS ONE 11 (2), e0149794 
Evans, D.E., Park, J.Y., Maxfield, N., and Drobes, D.J. (2009) 'Neurocognitive Variation in Smoking Behavior and Withdrawal: Genetic and Affective Moderators'. Genes, Brain and Behavior 8 (1), 86-96

Everitt, B.J. and Robbins, T.W. (2016) 'Drug Addiction: Updating Actions to Habits to Compulsions Ten Years On'. Annual Review of Psychology 67 (1), 23-50

Fagan, P. and Rigotti, N. A. (2009) 'Light and Intermittent Smoking: The Road Less Traveled'. Nicotine E Tobacco Research 11 (2), 107-110

Fagerström, K.-O. (2011) 'Determinants of Tobacco Use and Renaming the FTND to the Fagerström Test for Cigarette Dependence'. Nicotine \& Tobacco Research 14 (1), 7578

Faul, F., Erdfelder, E., Buchner, A., and Lang, A.-G. (2009) 'Statistical Power Analyses Using GPower 3.1: Tests for Correlation and Regression Analyses'. Behavior Research Methods 41 (4), 1149-1160

Field, M. and Cox, W.M. (2008) 'Attentional Bias in Addictive Behaviors: A Review of Its Development, Causes, and Consequences'. Drug and Alcohol Dependence 97 (1-2), $1-20$

Field, M., Heather, N., and Wiers, R.W. (2019) 'Indeed, Not Really a Brain Disorder: Implications for Reductionist Accounts of Addiction'. Behavioral and Brain Sciences 42, e9

Field, M. and Kersbergen, I. (2019) 'Are Animal Models of Addiction Useful?'. Addiction $115(1), 6-12$

Field, M., Mogg, K., and Bradley, B.P. (2004) 'Eye Movements to Smoking-Related Cues: Effects of Nicotine Deprivation'. Psychopharmacology 173 (1-2), 116-23 
Field, M., Mogg, K., and Bradley, B.P. (2005) 'Alcohol Increases Cognitive Biases for Smoking Cues in Smokers'. Psychopharmacology 180 (1), 63-72

Field, M., Mogg, K., and Bradley, B.P. (2006) 'Automaticity of Smoking Behaviour: The Relationship between Dual-Task Performance, Daily Cigarette Intake and Subjective Nicotine Effects'. Journal of Psychopharmacology 20 (6), 799-805

Field, M., Mogg, K., Zetteler, J., and Bradley, B.P. (2004) 'Attentional Biases for Alcohol Cues in Heavy and Light Social Drinkers: The Roles of Initial Orienting and Maintained Attention'. Psychopharmacology 176 (1), 88-93

Field, M., Munafó, M.R., and Franken, I.H.A. (2009) 'A Meta-Analytic Investigation of the Relationship between Attentional Bias and Subjective Craving in Substance Abuse'. Psychological Bulletin 135 (4), 589-607

Field, M., Werthmann, J., Franken, I.H.A., Hofmann, W., Hogarth, L., and Roefs, A. (2016) 'The Role of Attentional Bias in Obesity and Addiction'. Health Psychology 35 (8), 767-780

Frank, D.W., Cinciripini, P.M., Deweese, M.M., Karam-Hage, M., Kypriotakis, G., Lerman, C., Robinson, J.D., Tyndale, R.F., Vidrine, D.J., and Versace, F. (2019) 'Toward Precision Medicine for Smoking Cessation: Developing a Neuroimaging-Based Classification Algorithm to Identify Smokers at Higher Risk for Relapse'. Nicotine \& Tobacco Research [online] available from https://academic . oup.com/ntr/advance-article-abstract/ doi/10.1093/ntr/ntz211/5625395?redirectedFrom=fulltext [15March2020]

Franken, I.H.A., van Strien, J.W., and Kuijpers, I. (2010) 'Evidence for a Deficit in the Salience Attribution to Errors in Smokers'. Drug and Alcohol Dependence 106 (2-3), 181185

Franken, I.H.A. and van de Wetering, B.J.M. (2015) 'Bridging the Gap between the Neurocognitive Lab and the Addiction Clinic'. Addictive Behaviors 44, 108-114 
Fried, P.A., Watkinson, B., and Gray, R. (2006) 'Neurocognitive Consequences of Cigarette Smoking in Young Adults: A Comparison with Pre-Drug Performance'. Neurotoxicology and Teratology 28 (4), 517-525

Galván, A., Poldrack, R.A., Baker, C.M., McGlennen, K.M., and London, E.D. (2011) 'Neural Correlates of Response Inhibition and Cigarette Smoking in Late Adolescence'. Neuropsychopharmacology 36 (5), 970-978

Gantiva, C., Sotaquirá, M., Marroquín, M., Carné, C., Parada, L., and Muñoz, M.A. (2019) 'Size Matters in the Case of Graphic Health Warnings: Evidence from Physiological Measures'. Addictive Behaviors 92, 64-68

García-rodríguez, O., Blanco, C., Wall, M.M., Wang, S., Jin, C.J., and Kendler, K.S. (2014) 'Toward a Comprehensive Developmental Model of Smoking Initiation and Nicotine Dependence'. Drug and Alcohol Dependence 144, 160-169

Germine, L., Nakayama, K., Duchaine, B.C., Chabris, C.F., Chatterjee, G., and Wilmer, J.B. (2012) 'Is the Web as Good as the Lab? Comparable Performance from Web and Lab in Cognitive/Perceptual Experiments'. Psychonomic Bulletin E Review 19 (5), 847857

Goldstein, R.Z. and Volkow, N.D. (2002) 'Drug Addiction and Its Underlying Neurobiological Basis: Neuroimaging Evidence for the Involvement of the Frontal Cortex'. American Journal of Psychiatry 159 (10), 1642-1652

Goldstein, R.Z. and Volkow, N.D. (2011) 'Dysfunction of the Prefrontal Cortex in Addiction: Neuroimaging Findings and Clinical Implications'. Nature Reviews: Neuroscience $12(11), 652-69$

Gosling, S.D. and Mason, W. (2015) 'Internet Research in Psychology'. Annual Review of Psychology 66 (1), 877-902 
Goh, J.X., Hall, J.A., and Rosenthal, R. (2016) 'Mini Meta-Analysis of Your Own Studies: Some Arguments on Why and a Primer on How: Mini Meta-Analysis'. Social and Personality Psychology Compass 10 (10), 535-549

Goudriaan, A.E., De Ruiter, M.B., Van Den Brink, W., Oosterlaan, J., and Veltman, D.J. (2010) 'Brain Activation Patterns Associated with Cue Reactivity and Craving in Abstinent Problem Gamblers, Heavy Smokers and Healthy Controls: An FMRI Study: Neuroimaging Cue Reactivity in Problem Gamblers and Smokers'. Addiction Biology $15(4), 491-503$

Gramfort, A., Luessi, M., Larson, E., Engemann, D.A., Strohmeier, D., Brodbeck, C., Goj, R., Jas, M., Brooks, T., Parkkonen, L., and Hämäläinen, M. (2013) 'MEG and EEG Data Analysis with MNE-Python'. Frontiers in Neuroscience 7, 1-13

Greenland, S., Senn, S.J., Rothman, K.J., Carlin, J.B., Poole, C., Goodman, S.N., and Altman, D.G. (2016) 'Statistical Tests, P Values, Confidence Intervals, and Power: A Guide to Misinterpretations'. European Journal of Epidemiology 31 (4), 337-350

Haddock, K.C., Lando, H., Klesges, R.C., Talcott, G.W., and Renaud, E.A. (1999) 'A Study of the Psychometric and Predictive Properties of the Fagerström Test for Nicotine Dependence in a Population of Young Smokers'. Nicotine E Tobacco Research 1, $59-66$

Haight, J., Dickter, C.L., and Forestell, C.A. (2012) 'A Comparison of Daily and Occasional Smokers' Implicit Affective Responses to Smoking Cues'. Addictive Behaviors 37 (3), 234-239

Hakulinen, C., Hintsanen, M., Munafó, M.R., Virtanen, M., Kivimäki, M., Batty, G.D., and Jokela, M. (2015) 'Personality and Smoking: Individual-Participant Meta-Analysis of Nine Cohort Studie'. Addiction 110 (11), 1844-1852 
Hämmerer, D., Li, S.-C., Völkle, M., Müller, V., and Lindenberger, U. (2013) 'A Lifespan Comparison of the Reliability, Test-Retest Stability, and Signal-to-Noise Ratio of Event-Related Potentials Assessed during Performance Monitoring: Lifespan Differences in ERP Reliabilities'. Psychophysiology 50 (1), 111-123

Harakeh, Z., de Sonneville, L., van den Eijnden, R.J.J.M., Huizink, A.C., Reijneveld, S.A., Ormel, J., Verhulst, F.C., Monshouwer, K., and Vollebergh, W.A.M. (2012) 'The Association between Neurocognitive Functioning and Smoking in Adolescence: The TRAILS Study'. Neuropsychology 26 (5), 541-550

Harmsen, H., Bischof, G., Brooks, A., Hohagen, F., and Rumpf, H.-J. (2006) 'The Relationship between Impaired Decision-Making, Sensation Seeking and Readiness to Change in Cigarette Smokers'. Addictive Behaviors 31 (4), 581-592

Heatherton, T.F., Kozlowski, L.T., Frecker, R.C., and Fagerström, K.O. (1991) 'The Fagerström Test for Nicotine Dependence: A Revision of the Fagerström Tolerance Questionnaire'. British Journal of Addiction 86 (9), 1119-27

Hecht, S.S. (2010) 'Tobacco Carcinogenesis: Mechanisms and Biomarkers'. in Tobacco: Science, Policy, and Public Health. ed. by Boyle, P., Gray, N., Henningfield, J., Seffrin, J. and Zatonski, W. Oxford: Oxford University Press, 127-154

Hedge, C., Powell, G., and Sumner, P. (2018) 'The Reliability Paradox: Why Robust Cognitive Tasks Do Not Produce Reliable Individual Differences'. Behavior Research Methods 50 (3), 1166-1186

Henningfield, J.E. and Benowitz, N.L. (2010) 'Pharmacology of Tobacco Addiction'. in Tobacco: Science, Policy, and Public Health. ed. by Boyle, P., Gray, N., Henningfield, J.E., Seffrin, J. and Zatonski, W. Oxford: Oxford University Press, 155-170

Henson, R.K. (2001) 'Understanding Internal Consistency Reliability Estimates: A Conceptual Primer on Coefficient Alpha'. Measurement and Evaluation in Counseling 
and Development 34 (3), 177-189

Herbec, A., Brown, J., and West, R. (2014) 'Non-Daily Smoking In England: Addressing Common Misconceptions'. Smoking in Britain 1 (5), 1-13

Herman, C.P. (1974) 'External and Internal Cues as Determinants of the Smoking Behavior of Light and Heavy Smokers'. Journal of Personality and Social Psychology 30 (5), $664-672$

Hester, R. and Luijten, M. (2014) 'Neural Correlates of Attentional Bias in Addiction'. CNS spectrums 19 (3), 231-8

Heyman, G.M. and Gibb, S.P. (2006) 'Delay Discounting in College Cigarette Chippers'. Behavioural Pharmacology 17 (8), 669-679

Hilbig, B.E. (2016) 'Reaction Time Effects in Lab-versus Web-Based Research: Experimental Evidence'. Behavior Research Methods 48 (4), 1718-1724

Hoeppner, B.B., Bidwell, L.C., Colby, S.M., and Barnett, N.P. (2014) 'Smoking Patterns and Their Relationship to Drinking among First-Year College Students'. Nicotine $\mathcal{E}$ Tobacco Research 16 (6), 743-752

Hoffmann, S. and Falkenstein, M. (2008) 'The Correction of Eye Blink Artefacts in the EEG: A Comparison of Two Prominent Methods'. PLoS ONE 3 (8), 1-11

Hogarth, L. and Chase, H.W. (2012) 'Evaluating Psychological Markers for Human Nicotine Dependence: Tobacco Choice, Extinction, and Pavlovian-to-Instrumental Transfer'. Experimental and Clinical Psychopharmacology 20 (3), 213-224

Hogarth, L., Chase, H.W., and Baess, K. (2012) 'Impaired Goal-Directed Behavioural Control in Human Impulsivity'. Quarterly Journal of Experimental Psychology 65 (2), 305-316 
Hogarth, L.C., Mogg, K., Bradley, B.P., Duka, T., and Dickinson, A. (2003) 'Attentional Orienting towards Smoking-Related Stimuli'. Behavioural Pharmacology 14 (2), 153-160

Holm, S. (1979) 'A Simple Sequentially Rejective Multiple Test Procedure'. Scandinavian Journal of Statistics 6 (2), 65-70

Houston, R.J. and Schlienz, N.J. (2018) 'Event-Related Potentials as Biomarkers of Behavior Change Mechanisms in Substance Use Disorder Treatment'. Biological Psychiatry: Cognitive Neuroscience and Neuroimaging 3 (1), 30-40

Husten, C.G. (2009) 'How Should We Define Light or Intermittent Smoking? Does It Matter?'. Nicotine E Tobacco Research 11 (2), 111-121

Ip, C.-T., Ganz, M., Ozenne, B., Sluth, L.B., Gram, M., Viardot, G., l'Hostis, P., Danjou, P., Knudsen, G.M., and Christensen, S.R. (2018) 'Pre-Intervention Test-Retest Reliability of EEG and ERP over Four Recording Intervals'. International Journal of Psychophysiology $134,30-43$

Jasinska, A.J., Stein, E.A., Kaiser, J., Naumer, M.J., and Yalachkov, Y. (2014) 'Factors Modulating Neural Reactivity to Drug Cues in Addiction: A Survey of Human Neuroimaging Studies'. Neuroscience \& Biobehavioral Reviews 38, 1-16

JASP Team (2019). JASP (Version 0.11.1)[Computer software]

Jemal, A., Bray, F., Ferlay, J., Ward, E., and Forman, D. (2011) 'Global Cancer Statistics'. CA: A Cancer Journal for Clinicians 61, 69-90

Johnson, M.W., Bickel, W.K., and Baker, F. (2007) 'Moderate Drug Use and Delay Discounting: A Comparison of Heavy, Light, and Never Smokers'. Experimental and Clinical Psychopharmacology 15 (2), 187-194 
Jones, A., Christiansen, P., and Field, M. (2018a) 'Failed Attempts to Improve the Reliability of the Alcohol Visual Probe Task Following Empirical Recommendations'. Psychology of Addictive Behaviors 32 (8), 922-932

Jones, A., Di Lemma, L.C.G., Robinson, E., Christiansen, P., Nolan, S., Tudur-Smith, C., and Field, M. (2016) 'Inhibitory Control Training for Appetitive Behaviour Change: A Meta-Analytic Investigation of Mechanisms of Action and Moderators of Effectiveness'. Appetite 97, 16-28

Jones, A., McGrath, E., Robinson, E., Houben, K., Nederkoorn, C., and Field, M. (2018b) 'A Randomized Controlled Trial of Inhibitory Control Training for the Reduction of Alcohol Consumption in Problem Drinkers'. Journal of Consulting and Clinical Psychology 86 (12), 991

Kale, D., Stautz, K., and Cooper, A. (2018) 'Impulsivity Related Personality Traits and Cigarette Smoking in Adults: A Meta-Analysis Using the UPPS-P Model of Impulsivity and Reward Sensitivity'. Drug and Alcohol Dependence 185, 149-167

Kalkhoran, S., Chang, Y., and Rigotti, N.A. (2020) 'Electronic Cigarette Use and Cigarette Abstinence Over 2 Years Among U.S. Smokers in the Population Assessment of Tobacco and Health Study'. Nicotine \& Tobacco Research 22 (5), 728-733

Kalman, D. (2002) 'The Subjective Effects of Nicotine: Methodological Issues, A Review of Experimental Studies, and Recommendations for Future Research'. Nicotine $\mathcal{E}$ Tobacco Research 4 (1), 25-70

Kang, O.-S., Chang, D.-S., Jahng, G.-H., Kim, S.-Y., Kim, H., Kim, J.-W., Chung, S.-Y., Yang, S.-I., Park, H.-J., Lee, H., and Chae, Y. (2012) 'Individual Differences in SmokingRelated Cue Reactivity in Smokers: An Eye-Tracking and fMRI Study'. Progress in Neuropsychopharmacology \& Biological Psychiatry 38 (2), 285-93

Kappenman, E.S. and Luck, S.J. (2010) 'The Effects of Electrode Impedance on Data 
Quality and Statistical Significance in ERP Recordings'. Psychophysiology 47 (5), 888904

Kassel, J.D., Stroud, L.R., and Paronis, C.A. (2003) 'Smoking, Stress, and Negative Affect: Correlation, Causation, and Context across Stages of Smoking'. Psychological Bulletin 129 (2), 270-304

Kendler, K.S., Schmitt, E., Aggen, S.H., and Prescott, C.A. (2008) 'Genetic and Environmental Influences on Alcohol, Caffeine, Cannabis, and Nicotine Use from Early Adolescence to Middle Adulthood'. Archives of General Psychiatry 65 (6), 675-682

Knott, V.J., Naccache, L., Cyr, E., Fisher, D.J., McIntosh, J.F., Millar, A.M., and Villeneuve, C.M. (2008) 'Craving-Induced EEG Reactivity in Smokers: Effects of Mood Induction, Nicotine Dependence and Gender'. Neuropsychobiology 58 (3-4), 187-199

Kok, A., Ramautar, J.R., De Ruiter, M.B., Band, G.P.H., and Ridderinkhof, K.R. (2004) 'ERP Components Associated with Successful and Unsuccessful Stopping in a StopSignal Task'. Psychophysiology 41 (1), 9-20

Koo, T. K., and Li, M. Y. (2016) 'A guideline of selecting and reporting intraclass correlation coefficients for reliability research'. Journal of Chiropractic Medicine, 15 (2), 155163

Koontz, J.S., Harris, K.J., Okuyemi, K.S., Mosier, M.C., Grobe, J., Nazir, N., and Ahluwalia, J.S. (2010) 'Healthcare Providers' Treatment of College Smokers'. Journal of American College Health 53 (3), 117-126

Koster, E.H.W., Crombez, G., Verschuere, B., and De Houwer, J. (2004) 'Selective Attention to Threat in the Dot Probe Paradigm: Differentiating Vigilance and Difficulty to Disengage'. Behaviour Research and Therapy 42 (10), 1183-1192 
Kotz, D., Fidler, J., and West, R. (2012) 'Very Low Rate and Light Smokers: Smoking Patterns and Cessation-Related Behaviour in England, 2006-11'. Addiction 107 (5), 995-1002

Krishnan-Sarin, S., Reynolds, B., Duhig, A.M., Smith, A., Liss, T., McFetridge, A., Cavallo, D.A., Carroll, K.M., and Potenza, M.N. (2007) 'Behavioral Impulsivity Predicts Treatment Outcome in a Smoking Cessation Program for Adolescent Smokers'. Drug and Alcohol Dependence 88 (1), 79-82

Kruijt, A.-W., Field, A.P., and Fox, E. (2016) 'Capturing Dynamics of Biased Attention: Are New Attention Variability Measures the Way Forward?'. PLoS ONE 11 (11), $\mathrm{e} 0166600$

Lakens, D. (2013) 'Calculating and Reporting Effect Sizes to Facilitate Cumulative Science: A Practical Primer for T-Tests and ANOVAs'. Frontiers in Psychology 4, 1-12

Lakens, D. (2014) 'Performing High-Powered Studies Efficiently with Sequential Analyses'. European Journal of Social Psychology 44 (7), 701-710

Lang, P. J., Bradley, MM., and Cuthbert, B. N. (2008). International affective picture system (IAPS): Affective ratings of pictures and instruction manual. Technical Report A-8. Gainesville: University of Florida.

Larson, M.J., Baldwin, S.A., Good, D.A., and Fair, J.E. (2010) 'Temporal Stability of the Error-Related Negativity (ERN) and Post-Error Positivity (Pe): The Role of Number of Trials: ERN Test-Retest Reliability'. Psychophysiology 47, 1167-1171

Lasser, K., Boyd, J.W., Woolhandler, S., Himmelstein, D.U., McCormick, D., and Bor, D.H. (2000) 'Smoking and Mental Illness: A Population-Based Prevalence Study'. JAMA $284(20), 2606-2610$

Lawn, W., Freeman, T.P., East, K., Gaule, A., Aston, E.R., Bloomfield, M.A.P., Das, 
R.K., Morgan, C.J.A., and Curran, H.V. (2018) 'The Acute Effects of a Dopamine D3 Receptor Preferring Agonist on Motivation for Cigarettes in Dependent and Occasional Cigarette Smokers'. Nicotine E Tobacco Research 20 (7), 800-809

Lawn, W., Freeman, T.P., Hindocha, C., Mokrysz, C., Das, R.K., Morgan, C.J.A., and Curran, H.V. (2015) 'The Effects of Nicotine Dependence and Acute Abstinence on the Processing of Drug and Non-Drug Rewards'. Psychopharmacology 232 (14), 2503-2517

Levinson, A.H., Campo, S., Gascoigne, J., Jolly, O., Zakharyan, A., and Tran, Z.V. (2007) 'Smoking, but Not Smokers: Identity among College Students Who Smoke Cigarettes'. Nicotine \& Tobacco Research 9 (8), 845-852

Levy, D.E., Biener, L., and Rigotti, N.A. (2009) 'The Natural History of Light Smokers: A Population-Based Cohort Study'. Nicotine E Tobacco Research 11 (2), 156-163

Leys, C., Ley, C., Klein, O., Bernard, P., and Licata, L. (2013) 'Detecting Outliers: Do Not Use Standard Deviation around the Mean, Use Absolute Deviation around the Median'. Journal of Experimental Social Psychology 49 (4), 764-766

Light, G.A., Williams, L.E., Minow, F., Sprock, J., Rissling, A., Sharp, R., Swerdlow, N.R., and Braff, D.L. (2010) 'Electroencephalography (EEG) and Event-Related Potentials (ERPs) with Human Participants'. Current Protocols in Neuroscience 52 (1), 6.25.16.25 .24

Løchen, M.-L., Gram, I.T., Mannsverk, J., Mathiesen, E.B., Njølstad, I., Schirmer, H., Wilsgaard, T., and Jacobsen, B.K. (2017) 'Association of Occasional Smoking with Total Mortality in the Population-Based Tromsø Study, 2001-2015'. BMJ Open 7 (12), 1-6

Luck, S.J. and Gaspelin, N. (2017) 'How to Get Statistically Significant Effects in Any ERP Experiment (and Why You Shouldn't)'. Psychophysiology 54, 146-157 
Luijten, M., Kleinjan, M., and Franken, I.H.A. (2016) 'Event-Related Potentials Reflecting Smoking Cue Reactivity and Cognitive Control as Predictors of Smoking Relapse and Resumption'. Psychopharmacology 233 (15-16), 2857-2868

Luijten, M., Littel, M., and Franken, I.H.A. (2011a) 'Deficits in Inhibitory Control in Smokers during a Go/Nogo Task: An Investigation Using Event-Related Brain Potentials'. PLOS ONE 6 (4)

Luijten, M., O'Connor, D.A., Rossiter, S., Franken, I.H.A., and Hester, R. (2013) 'Effects of Reward and Punishment on Brain Activations Associated with Inhibitory Control in Cigarette Smokers: Reward, Punishment and Inhibition in Smokers'. Addiction 108 (11), 1969-1978

Luijten, M., Van Meel, C.S., and Franken, I.H.A. (2011b) 'Diminished Error Processing in Smokers during Smoking Cue Exposure'. Pharmacology Biochemistry and Behavior 97 (3), $514-520$

Luijten, M., Machielsen, M.W.J., Veltman, D.J., Hester, R., de Haan, L., and Franken, I.H.A. (2014) 'Systematic Review of ERP and fMRI Studies Investigating Inhibitory Control and Error Processing in People with Substance Dependence and Behavioural Addictions'. Journal of Psychiatry and Neuroscience 39 (3), 149-169

MacCallum, R.C., Zhang, S., Preacher, K.J., and Rucker, D.D. (2002) 'On the Practice of Dichotomization of Quantitative Variables'. Psychological Methods 7 (1), 19-40

Marhe, R., Luijten, M., and Franken, I.H.A. (2014) 'The Clinical Relevance of Neurocognitive Measures in Addiction'. Frontiers in Psychiatry 4 (185), 1-7

Mathôt, S., Schreij, D., and Theeuwes, J. (2012) 'OpenSesame: An Open-Source, Graphical Experiment Builder for the Social Sciences'. Behavior Research Methods 44 (2), 314324 
Maynard, O.M., Brooks, J.C.W., Munafó, M.R., and Leonards, U. (2017) 'Neural Mechanisms Underlying Visual Attention to Health Warnings on Branded and Plain Cigarette Packs'. Addiction $112(4), 662-672$

McGrath, D.S., Peloquin, M.P., Ferdinand, J.C., and Barrett, S.P. (2015) 'Acute Effects of Nicotine on Alcohol Cue-Reactivity in Nondependent and Dependent Smokers'. Experimental and Clinical Psychopharmacology 23 (1), 29-36

Mogg, K. and Bradley, B.P. (2002) 'Selective Processing of Smoking-Related Cues in Smokers: Manipulation of Deprivation Level and Comparison of Three Measures of Processing Bias'. Journal of Psychopharmacology 16 (4), 385-392

Mogg, K., Bradley, B.P., Field, M., and De Houwer, J. (2003) 'Eye Movements to SmokingRelated Pictures in Smokers: Relationship between Attentional Biases and Implicit and Explicit Measures of Stimulus Valence'. Addiction 98 (6), 825-836

Mogg, K., Field, M., and Bradley, B.P. (2005) 'Attentional and Approach Biases for Smoking Cues in Smokers: An Investigation of Competing Theoretical Views of Addiction'. Psychopharmacology 180, 333-341

Moher, D., Shamseer, L., Clarke, M., Ghersi, D., Liberati, A., Petticrew, M., Shekelle, P., and Stewart, L.A. (2015) 'Preferred Reporting Items for Systematic Review and MetaAnalysis Protocols (PRISMA-P) 2015 Statement'. Systematic Reviews 4 (1), 1

Moodie, C., Hoek, J., Scheffels, J., Gallopel-Morvan, K., and Lindorff, K. (2018) 'Plain Packaging: Legislative Differences in Australia, France, the UK, New Zealand and Norway, and Options for Strengthening Regulations'. Tobacco Control 28, 485-492

Moshontz, H., Campbell, L., Ebersole, C.R., IJzerman, H., Urry, H.L., Forscher, P.S., Grahe, J.E., McCarthy, R.J., Musser, E.D., Antfolk, J., Castille, C.M., Evans, T.R., Fiedler, S., Flake, J.K., Forero, D.A., Janssen, S.M.J., Keene, J.R., Protzko, J., Aczel, B., Solas, S.Á., Ansari, D., Awlia, D., Baskin, E., Batres, C., Borras-Guevara, M.L., Brick, 
C., Chandel, P., Chatard, A., Chopik, W.J., Clarance, D., Coles, N.A., Corker, K.S., Dixson, B.J.W., Dranseika, V., Dunham, Y., Fox, N.W., Gardiner, G., Garrison, S.M., Gill, T., Hahn, A.C., Jaeger, B., Ka'már, P., Kaminski, G., Kanske, P., Kekecs, Z., Kline, M., Koehn, M.A., Kujur, P., Levitan, C.A., Miller, J.K., Okan, C., Olsen, J., OviedoTrespalacios, O., Özdo?ru, A.A., Pande, B., Parganiha, A., Parveen, N., Pfuhl, G., Pradhan, S., Ropovik, I., Rule, N.O., Saunders, B., Schei, V., Schmidt, K., Singh, M.M., Sirota, M., Steltenpohl, C.N., Stieger, S., Storage, D., Sullivan, G.B., Szabelska, A., Tamnes, C.K., Vadillo, M.A., Valentova, J.V., Vanpaemel, W., Varella, M.A.C., Vergauwe, E., Verschoor, M., Vianello, M., Voracek, M., Williams, G.P., Wilson, J.P., Zickfeld, J.H., Arnal, J.D., Aydin, B., Chen, S.-C., DeBruine, L.M., Fernandez, A.M., Horstmann, K.T., Isager, P.M., Jones, B., Kapucu, A., Lin, H., Mensink, M.C., Navarrete, G., Silan, M.A., and Chartier, C.R. (2018) 'The Psychological Science Accelerator: Advancing Psychology Through a Distributed Collaborative Network'. Advances in Methods and Practices in Psychological Science 1 (4), 501-515

Mostofsky, S.H., Schafer, J.G.B., Abrams, M.T., Goldberg, M.C., Flower, A.A., Boyce, A., Courtney, S.M., Calhoun, V.D., Kraut, M.A., Denckla, M.B., and Pekar, J.J. (2003) 'fMRI Evidence That the Neural Basis of Response Inhibition Is Task-Dependent'. Cognitive Brain Research 17 (2), 419-430

Motschman, C.A. and Tiffany, S.T. (2016) 'Cognitive Regulation of Smoking Behavior within a Cigarette: Automatic and Nonautomatic Processes'. Psychology of Addictive Behaviors 30 (4), 494-499

Munafó, M.R., Zetteler, J.I., and Clark, T.G. (2007) 'Personality and Smoking Status: A Meta-Analysis'. Nicotine E Tobacco Research 9 (3), 405-413

Nees, F. (2014) 'The Nicotinic Cholinergic System Function in the Human Brain'. Neuropharmacology 96, 289-301

Nesic, J., Rusted, J., Duka, T., and Jackson, A. (2011) 'Degree of Dependence Influences the Effect of Smoking on Cognitive Flexibility'. Pharmacology Biochemistry and 
Behavior 98 (3), 376-384

Nestor, L., McCabe, E., Jones, J., Clancy, L., and Garavan, H. (2011) 'Differences in 'Bottom-up' and 'Top-down' Neural Activity in Current and Former Cigarette Smokers: Evidence for Neural Substrates Which May Promote Nicotine Abstinence through Increased Cognitive Control'. NeuroImage 56 (4), 2258-2275

Neyman, J. (1977) 'Frequentist Probability and Frequentist Statistics'. Synthese 36(1), 97-131

Okuyemi, K.S., Harris, K.J., Scheibmeir, M., Choi, W.S., Powell, J., and Ahluwalia, J.S. (2002) 'Light Smokers: Issues and Recommendations'. Nicotine \& Tobacco Research 4 (Supplement 2), S103-S112

Olvet, D.M. and Hajcak, G. (2009) 'Reliability of Error-Related Brain Activity'. Brain Research 1284, 89-99

Open Science Collaboration (2015) 'Estimating the Reproducibility of Psychological Science'. Science 349 (6251), 1-8

Oppenheimer, D.M., Meyvis, T., and Davidenko, N. (2009) 'Instructional Manipulation Checks: Detecting Satisficing to Increase Statistical Power'. Journal of Experimental Social Psychology 45 (4), 867-872

Overbeek, T.J.M., Nieuwenhuis, S., and Ridderinkhof, K.R. (2005) 'Dissociable Components of Error Processing: On the Functional Significance of the Pe Vis-a-Vis the ERN/Ne'. Journal of Psychophysiology 19 (4), 319-329

Paelecke-Habermann, Y., Paelecke, M., Giegerich, K., Reschke, K., and Kübler, A. (2013) 'Implicit and Explicit Reward Learning in Chronic Nicotine Use'. Drug and Alcohol Dependence 129 (1-2), 8-17 
Parsons, S., Kruijt, A.-W., and Fox, E. (2019) 'Psychological Science Needs a Standard Practice of Reporting the Reliability of Cognitive-Behavioral Measurements'. Advances in Methods and Practices in Psychological Science 2 (4), 378-395

Parvaz, M.A., Alia-Klein, N., Woicik, P.A., Volkow, N.D., and Goldstein, R.Z. (2011) 'Neuroimaging for Drug Addiction and Related Behaviors'. Reviews in the Neurosciences $22(6), 609-624$

Peirce, J.W. (2009) 'Generating Stimuli for Neuroscience Using PsychoPy'. Frontiers in Neuroinformatics 2 (10), 1-8

Piasecki, T.M., Fleming, K.A., Trela, C.J., and Bartholow, B.D. (2017) 'P3 Event-Related Potential Reactivity to Smoking Cues: Relations with Craving, Tobacco Dependence, and Alcohol Sensitivity in Young Adult Smokers'. Psychology of Addictive Behaviors 31 $(1), 61-72$

Pierce, J.P., White, M.M., and Messer, K. (2009) 'Changing Age-Specific Patterns of Cigarette Consumption in the United States, 1992 - 2002: Association with SmokeFree Homes and State-Level Tobacco Control Activity'. Nicotine E Tobacco Research 11 (2), 171-177

Piper, M.E., Bolt, D.M., Kim, S.-Y., Japuntich, S.J., Smith, S.S., Niederdeppe, J., Cannon, D.S., and Baker, T.B. (2008) 'Refining the Tobacco Dependence Phenotype Using the Wisconsin Inventory of Smoking Dependence Motives'. Journal of Abnormal Psychology 117 (4), 747-61

Poltavski, D.V. and Weatherly, J.N. (2013) 'Delay and Probability Discounting of Multiple Commodities in Smokers and Never-Smokers Using Multiple-Choice Tasks'. Behavioural Pharmacology 24 (8), 659-667

Pomerleau, C.S., Carton, S.M., Lutzke, M.L., Flessland, K.A., and Pomerleau, O.F. (1994) 'Reliability of the Fagerström Tolerance Questionnaire and the Fagerström Test 
for Nicotine Dependence'. Addictive Behaviors 19 (1), 33-39

Powell, J., Dawkins, L., West, R., Powell, J., and Pickering, A. (2010) 'Relapse to Smoking during Unaided Cessation: Clinical, Cognitive and Motivational Predictors'. Psychopharmacology 212 (4), 537-549

Price, R.B., Kuckertz, J.M., Siegle, G.J., Ladouceur, C.D., Silk, J.S., Ryan, N.D., Dahl, R.E., and Amir, N. (2015) 'Empirical Recommendations for Improving the Stability of the Dot-Probe Task in Clinical Research'. Psychological Assessment 27 (2), 365-376

R Core Team (2019). R: A language and environment for statistical computing. R Foundation for Statistical Computing, Vienna, Austria. https://www.R-project.org/.

Ramo, D.E., Hall, S.M., and Prochaska, J.J. (2011) 'Reliability and Validity of SelfReported Smoking in an Anonymous Online Survey with Young Adults'. Health Psychology 30 (6), 693-701

Rass, O., Ahn, W.-Y., and O'Donnell, B.F. (2016) 'Resting-State EEG, Impulsiveness, and Personality in Daily and Nondaily Smokers'. Clinical Neurophysiology 127 (1), 409418

Rass, O., Fridberg, D.J., and O'Donnell, B.F. (2014) 'Neural Correlates of Performance Monitoring in Daily and Intermittent Smokers'. Clinical Neurophysiology 125 (7), 14171426

Redick, T.S., Calvo, A., Gay, C.E., and Engle, R.W. (2011) 'Working Memory Capacity and Go/No-Go Task Performance: Selective Effects of Updating, Maintenance, and Inhibition'. Journal of Experimental Psychology: Learning, Memory, and Cognition 37 (2), 308-324

Retzler, C., Shiraj, N., and Retzler, J. (2019) 'Eye Movement Data Reveal Increased 
Attention to Combined Health Warnings on Cigarette Packs'. Drug and Alcohol Dependence $194,336-340$

Rezvanfard, M., Ekhtiari, H., Mokri, A., Djavid, G.E., and Kaviani, H. (2010) 'Psychological and Behavioral Traits in Smokers and Their Relationship with Nicotine Dependence Level'. Archives of Iranian Medicine 13 (5), 395-405

Ridderinkhof, K.R., Ramautar, J.R., and Wijnen, J.G. (2009) 'To Pe or Not to Pe: A P3-like ERP Component Reflecting the Processing of Response Errors'. Psychophysiology 46 (3), $531-538$

Rietdijk, W.J.R., Franken, I.H.A., and Thurik, A.R. (2014) 'Internal Consistency of Event-Related Potentials Associated with Cognitive Control: N2/P3 and ERN/Pe'. PLOS ONE 9 (7), 3-9

Robertson, L., Iosua, E., McGee, R., and Hancox, R.J. (2016) 'Non-Daily, Low-Rare Daily and High-Rate Daily Smoking in Young Adults: A 17 Year Follow-Up'. Nicotine $\mathcal{E}$ Tobacco Research 15 (5), 943-949

Robinson, T.E. and Berridge, K.C. (1993) 'The Neural Basis of Drug Craving: An Incentive-Sensitization Theory of Addiction'. Brain Research Reviews 18, 247-291

Robinson, T.E. and Berridge, K.C. (2003) 'Addiction'. Annual Review of Psychology 54, $25-53$

Robinson, T.E. and Berridge, K.C. (2008) 'The Incentive Sensitization Theory of Addiction: Some Current Issues'. Philosophical transactions of the Royal Society of London, Series B 363, 3137-3146

Rouder, J.N., Morey, R.D., Speckman, P.L., and Province, J.M. (2012) 'Default Bayes Factors for ANOVA Designs'. Journal of Mathematical Psychology 56 (5), 356-374 
Rubinstein, M.L., Rait, M.A, Sen, S., and Shiffman, S. (2014) 'Characteristics of Adolescent Intermittent and Daily Smokers'. Addictive Behaviors 39 (9), 1337-41

Russell, M.A.H. (1971) 'Cigarette Smoking: Natural History of a Dependence Disorder'. British Journal of Medical Psychology 44 (1), 1-16

Salemink, E., van den Hout, M.A., and Kindt, M. (2007) 'Selective Attention and Threat: Quick Orienting versus Slow Disengagement and Two Versions of the Dot Probe Task'. Behaviour Research and Therapy 45 (3), 607-615

Sayette, M.A., Martin, C.S., Wertz, J.M., Shiffman, S., and Perrott, M.A. (2001) 'A Multi-Dimensional Analysis of Cue-Elicited Craving in Heavy Smokers and Tobacco Chippers'. Addiction 96 (10), 1419-1432

Schäfer, T. and Schwarz, M.A. (2019) 'The Meaningfulness of Effect Sizes in Psychological Research: Differences Between Sub-Disciplines and the Impact of Potential Biases'. Frontiers in Psychology 10 (813), 1-13

Schane, R.E., Glantz, S.A., and Ling, P.M. (2009) 'Nondaily and Social Smoking an Increasingly Prevalent Pattern'. Archives of Internal Medicine 169 (19), 1742-1744

Schane, R.E., Ling, P.M., and Glantz, S. A. (2010) 'Health Effects of Light and Intermittent Smoking: A Review'. Circulation 121 (13), 1518-1522

Schauer, G.L., Malarcher, A.M., and Berg, C.J. (2014) 'Differences in Smoking and Cessation Characteristics among Adult Nondaily Smokers in the United States: Findings from the 2009-2010 National Adult Tobacco Survey'. Nicotine \& Tobacco Research 16 (1), $58-68$

Scheuermann, T.S., Nollen, N.L., Cox, L.S., Reitzel, L.R., Berg, C.J., Guo, H., Resnicow, K., and Ahluwalia, J.S. (2015) 'Smoking Dependence across the Levels of Cigarette Smoking in a Multiethnic Sample'. Addictive Behaviors 43, 1-6 
Schmukle, S.C. (2005) 'Unreliability of the Dot Probe Task'. European Journal of Personality 19, 595-605

Schoenmakers, T., Wiers, R.W., and Field, M. (2008) 'Effects of a Low Dose of Alcohol on Cognitive Biases and Craving in Heavy Drinkers'. Psychopharmacology 197 (1), $169-178$

Scholten, H., Scheres, A., de Water, E., Graf, U., Granic, I., and Luijten, M. (2019) 'Behavioral Trainings and Manipulations to Reduce Delay Discounting: A Systematic Review'. Psychonomic Bulletin \& Review 26 (6), 1803-1849

Schulte, M.H.J., Cousijn, J., den Uyl, T.E., Goudriaan, A.E., van den Brink, W., Veltman, D.J., Schilt, T., and Wiers, R.W. (2014) 'Recovery of Neurocognitive Functions Following Sustained Abstinence after Substance Dependence and Implications for Treatment'. Clinical Psychology Review 34 (7), 531-550

Sheffer, C., Mackillop, J., McGeary, J., Landes, R., Carter, L., Yi, R., Jones, B., Christensen, D., Stitzer, M., Jackson, L., and Bickel, W. (2012) 'Delay Discounting, Locus of Control, and Cognitive Impulsiveness Independently Predict Tobacco Dependence Treatment Outcomes in a Highly Dependent, Lower Socioeconomic Group of Smokers'. The American Journal on Addictions 21 (3), 221-232

Shiffman, S. (2009) 'Light and Intermittent Smokers: Background and Perspective'. Nicotine and Tobacco Research 11 (2), 122-125

Shiffman, S., Dunbar, M.S., Scholl, S.M., and Tindle, H.A. (2012a) 'Smoking Motives of Daily and Non-Daily Smokers: A Profile Analysis'. Drug and Alcohol Dependence 126 (3), 362-368

Shiffman, S., Ferguson, S.G., Dunbar, M.S., and Scholl, S.M. (2012b) 'Tobacco Dependence among Intermittent Smokers'. Nicotine \& Tobacco Research 14 (11), 1372-1381 
Shiffman, S., Tindle, H., Li, X., Scholl, S., Dunbar, M., and Mitchell-Miland, C. (2012c) 'Characteristics and Smoking Patterns of Intermittent Smokers'. Experimental and Clinical Psychopharmacology 20 (4), 264-277

Shiffman, S., Dunbar, M.S., and Benowitz, N.L. (2014a) 'A Comparison of Nicotine Biomarkers and Smoking Patterns in Daily and Nondaily Smokers'. Cancer Epidemiology Biomarkers E Prevention 23 (7), 1264-1272

Shiffman, S., Dunbar, M.S., Li, X., Scholl, S.M., Tindle, H.A., Anderson, S.J., and Ferguson, S.G. (2014b) 'Smoking Patterns and Stimulus Control in Intermittent and Daily Smokers'. PloS ONE 9 (3), e89911

Siddaway, A. P., Wood, A. M., and Hedges, L. V. (2019) 'How to Do a Systematic Review: A Best Practice Guide for Conducting and Reporting Narrative Reviews, MetaAnalyses, and Meta-Syntheses'. Annual Review of Psychology 70(1), 1-24

Simmons, J.P., Nelson, L.D., and Simonsohn, U. (2011) 'False-Positive Psychology: Undisclosed Flexibility in Data Collection and Analysis Allows Presenting Anything as Significant'. Psychological Science 22 (11), 1359-1366

Siqueira, L., Diab, M., Bodian, C., and Rolnitzky, L. (2000) 'Adolescents Becoming Smokers: The Roles of Stress and Coping Methods'. Journal of Adolescent Health 27 (6), 399-408

Smith, J.L., Jamadar, S., Provost, A.L., and Michie, P.T. (2013) 'Motor and Non-Motor Inhibition in the Go/NoGo Task: An ERP and FMRI Study'. International Journal of Psychophysiology 87 (3), 244-253

Smith, J.L., Mattick, R.P., Jamadar, S.D., and Iredale, J.M. (2014) 'Deficits in Behavioural Inhibition in Substance Abuse and Addiction: A Meta-Analysis'. Drug and Alcohol Dependence $145,1-33$ 
St. Helen, G., Benowitz, N.L., Ahluwalia, J.S., Tyndale, R.F., Addo, N., Gregorich, S.E., Pérez-Stable, E.J., and Cox, L.S. (2019) 'Black Light Smokers: How Nicotine Intake and Carcinogen Exposure Differ Across Various Biobehavioral Factors'. Journal of the National Medical Association 111 (5), 509-520

Stahl, S. M. (2013). Stahl's Essential Psychopharmacology. Cambridge: Cambridge University Press.

Steele, V., Anderson, N., Claus, E.D., Bernat, E.M., Rao, V., Assaf, M., Pearlson, G., Calhoun, V.D., and Kiehl, K.A. (2016) 'Neuroimaging Measures of Error-Processing: Extracting Reliable Signals from Event-Related Potentials and Functional Magnetic Resonance Imaging'. NeuroImage 132, 247-260

Stevens, H.A. (1976) 'Evidence That Suggests a Negative Association between Cigarette Smoking and Learning Performance'. Journal of Clinical Psychology 32 (4), 896-898

Stillwell, D.J. and Tunney, R.J. (2012) 'Effects of Measurement Methods on the Relationship between Smoking and Delay Reward Discounting: Link between Delay Discounting and Smoking'. Addiction 107 (5), 1003-1012

Sweitzer, M., Donny, E., Dierker, L., Flory, J., and Manuck, S. (2008) 'Delay Discounting and Smoking: Association with the Fagerström Test for Nicotine Dependence but Not Cigarettes Smoked per Day'. Nicotine E Tobacco Research 10 (10), 1571-1575

Szucs, D. and Ioannidis, J.P.A. (2017) 'Empirical Assessment of Published Effect Sizes and Power in the Recent Cognitive Neuroscience and Psychology Literature'. PLoS Biology 15 (3), e2000797

Tait, R., Martin-Iverson, M., Michie, P.T., and Dusci, L. (2000) 'The Effects of Cigarette Consumption on the Sternberg Visual Memory Search Paradigm'. Addiction 95 (3), $437-446$ 
Thigpen, N., Kappenman, E., and Keil, A. (2017) 'Assessing the Internal Consistency of the Event-Related Potential: An Example Analysis'. Psychophysiology 54 (1), 123-138

Thorgeirsson, T.E., Gudbjartsson, D.F., Surakka, I., Vink, J.M., Amin, N., Geller, F., Sulem, P., Rafnar, T., Esko, T., and Walter, S. (2010) 'Sequence Variants at CHRNB3CHRNA6 and CYP2A6 Affect Smoking Behavior'. Nature Genetics 42 (5), 448-453

Thun, M.J. and Henley, S.J. (2010) 'The Great Studies of Smoking and Disease in the Twentieth Century'. in Tobacco: Science, Policy, and Public Health. ed. by Boyle, P., Gray, N., Henningfield, J., Seffrin, J. and Zatonski, W. Oxford: Oxford University Press, 13-30

Tindle, H.A. and Shiffman, S. (2011) 'Smoking Cessation Behavior among Intermittent Smokers versus Daily Smokers'. American Journal of Public Health 101 (7), 1-3

Tong, E.K., Ong, M.K., Vittinghoff, E., and Pérez-Stable, E.J. (2006) 'Nondaily Smokers Should Be Asked and Advised to Quit'. American Journal of Preventive Medicine 30 (1), 23-30

Turetsky, B.I., Bilker, W.B., Siegel, S.J., Kohler, C.G., and Gur, R.E. (2009) 'The Profile of Auditory Information Processing Deficits in Schizophrenia'. Psychiatry Research $165(1-2), 27-37$

van Doorn, J., van den Bergh, D., Bohm, U., Dablander, F., Derks, K., Draws, T., Evans, N.J., Gronau, Q.F., Hinne, M., Kucharsky, S., Ly, A., Marsman, M., Matzke, D., Raj, A., Sarafoglou, A., Stefan, A., Voelkel, J.G., and Wagenmakers, E.-J. (2019) The JASP Guidelines for Conducting and Reporting a Bayesian Analysis. [online] available from https:/ /osf.io/yqxfr [15 March 2020]

Venugopalan, V.V., Casey, K.F., O’Hara, C., O’Loughlin, J., Benkelfat, C., Fellows, L.K., and Leyton, M. (2011) 'Acute Phenylalanine/Tyrosine Depletion Reduces Motivation to Smoke Cigarettes Across Stages of Addiction'. Neuropsychopharmacology 36 (12), 
$2469-2476$

Verbruggen, F. and Logan, G.D. (2009) 'Automatic and Controlled Response Inhibition: Associative Learning In The Go/No-Go and Stop-Signal Paradigm'. Journal of Experimental Psychology 137 (4), 649-672

Verdejo-García, A., Lawrence, A.J., and Clark, L. (2008) 'Impulsivity as a Vulnerability Marker for Substance-Use Disorders: Review of Findings from High-Risk Research, Problem Gamblers and Genetic Association Studies'. Neuroscience E Biobehavioral Reviews 32 (4), 777-810

Versace, F., Lam, C.Y., Engelmann, J.M., Robinson, J.D., Minnix, J.A., Brown, V.L., and Cinciripini, P.M. (2012) 'Beyond Cue Reactivity: Blunted Brain Responses to Pleasant Stimuli Predict Long-Term Smoking Abstinence: ERPs Predict Smoking Cessation'. Addiction Biology 17 (6), 991-1000

Volkow, N.D., Wang, G.J., Fowler, J.S., Tomasi, D., Telang, F., and Baler, R. (2010) 'Addiction: Decreased Reward Sensitivity and Increased Expectation Sensitivity Conspire to Overwhelm the Brain's Control Circuit'. BioEssays 32 (9), 748-755

Vollstädt-Klein, S., Loeber, S., Winter, S., Leménager, T., von der Goltz, C., Dinter, C., Koopmann, A., Wied, C., Winterer, G., and Kiefer, F. (2011) 'Attention Shift towards Smoking Cues Relates to Severity of Dependence, Smoking Behavior and Breath Carbon Monoxide'. European Addiction Research 17 (4), 217-24

Vosgerau, J., Simonsohn, U., Nelson, L.D., and Simmons, J.P. (2018) '99\% Impossible: A Valid, or Falsifiable, Internal Meta-Analysis'. Journal of Experimental Psychology: General 148(9), 1628-1639

Waechter, S., Nelson, A.L., Wright, C., Hyatt, A., and Oakman, J. (2014) 'Measuring Attentional Bias to Threat: Reliability of Dot Probe and Eye Movement Indices'. Cognitive Therapy and Research 38 (3), 313-333 
Wagge, J.R., Brandt, M.J., Lazarevic, L.B., Legate, N., Christopherson, C., Wiggins, B., and Grahe, J.E. (2019) 'Publishing Research With Undergraduate Students via Replication Work: The Collaborative Replications and Education Project'. Frontiers in Psychology 10,247

Wang, J.B., Olgin, J.E., Nah, G., Vittinghoff, E., Cataldo, J.K., Pletcher, M.J., and Marcus, G.M. (2018) 'Cigarette and E-Cigarette Dual Use and Risk of Cardiopulmonary Symptoms in the Health EHeart Study'. PLoS ONE 13 (7), 1-14.

Wardell, J.D., Dermody, S.S., Lindgren, K.P., Medina, A.M., and Hendershot, C.S. (2018) 'Implicit Alcohol and Smoking Associations among Young Adult Heavy Drinkers: Associations with Smoking Status and Alcohol-Cigarette Co-Use'. Motivation and Emotion $42(5), 682-690$

Ware, J.J. and Munafó, M. R. (2015) 'Genetics of Smoking Behaviour'. in The Neurobiology and Genetics of Nicotine and Tobacco. ed. by Balfour, D.J.K and Munafó, M.R. Cham: Springer, 19-36

Watson, D., Clark, L.A., and Tellegen, A. (1988) 'Development and Validation of Brief Measures of Positive and Negative Affect: The PANAS Scales'. Journal of Personality and Social Psychology 54 (6), 1063-1070

Weinberg, A. and Hajcak, G. (2011) 'Longer Term Test-Retest Reliability of ErrorRelated Brain Activity'. Psychophysiology 48 (10), 1420-1425

Wellman, R.J., Dugas, E.N., Dutczak, H., O’Loughlin, E.K., Datta, G.D., Lauzon, B., and O'Loughlin, J. (2016) 'Predictors of the Onset of Cigarette Smoking: A Systematic Review of Longitudinal Population-Based Studies in Youth'. American Journal of Preventive Medicine 51 (5), 767-778

Wessel, J.R., Danielmeier, C., and Ullsperger, M. (2011) 'Error Awareness Revisited: 
Accumulation of Multimodal Evidence from Central and Autonomic Nervous Systems'. Journal of Cognitive Neuroscience 23 (10), 3021-3036

Wessel, J.R. (2018) 'Prepotent Motor Activity and Inhibitory Control Demands in Different Variants of the Go/No-Go Paradigm'. Psychophysiology 55 (3), 1-14

West, R. (2009) 'The Multiple Facets of Cigarette Addiction and What They Mean for Encouraging and Helping Smokers to Stop'. COPD: Journal of Chronic Obstructive Pulmonary Disease 6, 277-283

West, R. (2017) 'Tobacco smoking: Health impact, prevalence, correlates and interventions'. Psychology \& Health, 32(8), 1018-1036

West, R. and Brown, J. (2013) Theory of Addiction. Chichester: John Wiley and Sons.

West, R., Beard, E., Kale, D., Kock, L. and Brown, J. (2020a) Trends in electronic cigarette use in England [online] available from http:/ / www.smokinginengland.info/latest-statistics / [24 October 2020]

West, R., Kale, D., Kock, L. and Brown, J. (2020b) Top-line findings on smoking in England from the Smoking Toolkit Study [online] available from http:/ / www.smokinginengland.info/lateststatistics/ [24 October 2020]

Witkiewitz, K., Desai, S.A., Steckler, G., Jackson, K.M., Bowen, S., Leigh, B.C., and Larimer, M.E. (2012) 'Concurrent Drinking and Smoking among College Students: An Event-Level Analysis'. Psychology of Addictive Behaviors 26 (3), 649-54

Woodman, G.F. (2010) 'A Brief Introduction to the Use of Event-Related Potentials (ERPs) in Studies of Perception and Attention'. Attention, Perception E Psychophysics, 72(8), 2031-2046

Woods, A.T., Velasco, C., Levitan, C.A., Wan, X., and Spence, C. (2015) 'Conducting 
Perception Research over the Internet: A Tutorial Review'. PeerJ 3, e1058

World Health Organisation (2015) Prevalence of Tobacco Smoking [online] available from http:/ /www.who.int/gho/tobacco/use/en/ [15 March 2020]

Wray, J.M., Gass, J.C., Miller, E.I., Wilkins, D.G., Rollins, D.E., and Tiffany, S.T. (2015) 'A Comparative Evaluation of Self-Report and Biological Measures of Cigarette Use in Nondaily Smokers'. Psychological Assessment 28 (9), 1043-1050

Yakir, A., Rigbi, A., Kanyas, K., Pollak, Y., Kahana, G., Karni, O., Eitan, R., Kertzman, S., and Lerer, B. (2007) 'Why Do Young Women Smoke? III. Attention and Impulsivity as Neurocognitive Predisposing Factors'. European Neuropsychopharmacology 17 (5), 339-351

Yücel, M., \& Lubman, D. (2007) 'Neurocognitive and neuroimaging evidence of behavioural dysregulation in human drug addiction: Implications for diagnosis, treatment and prevention'. Drug and Alcohol Review 26(1), 33-39

Zack, M., Belsito, L., Scher, R., Eissenberg, T., and Corrigall, W.A. (2001) 'Effects of Abstinence and Smoking on Information Processing in Adolescent Smokers'. Psychopharmacology 153 (2), 249-257

Zhao, X., Liu, X., Zan, X., Jin, G., and Maes, J.H.R. (2016) 'Male Smokers' and NonSmokers' Response Inhibition in Go/No-Go Tasks: Effect of Three Task Parameters'. PloS ONE 11 (8), e0160595

Zilverstand, A., Huang, A.S., Alia-Klein, N., and Goldstein, R.Z. (2018) 'Neuroimaging Impaired Response Inhibition and Salience Attribution in Human Drug Addiction: A Systematic Review'. Neuron 98 (5), 886-903

Zwaan, R.A., Pecher, D., Paolacci, G., Bouwmeester, S., Verkoeijen, P., Dijkstra, K., 
and Zeelenberg, R. (2017) 'Participant Nonnaiveté and the Reproducibility of Cognitive Psychology'. Psychonomic Bulletin \& Review 1-5 


\section{Appendix A}

\section{Systematic review search terms and}

\section{strategy}

For each database, each combination of terms for smoking groups and neurocognitive mechanisms would be combined. For example, the first combination would be all terms for light smokers and general cognitive labels:

("light smok*" or "non-daily smok*" or "intermittent smok*" or "social smok*" or "occasional smok" or "chipper" or "low frequency smok*" or "very light smok*" or "nondependent smok*") AND ("neurocognitive mechanis*" or "neurocognitive measure*" or "neuropsychological" or "neuroimaging" or "cogniti*" or "neural correlate" or "neurocognitive functioning").

This would identify articles containing any of the terms within each category, as long as one term was present from each category. This was repeated for each combination of categories listed below. Note the asterisk $\left({ }^{*}\right)$ allows the database to identify different endings to the word, so "light smok*" could identify "light smokers", "light smoker", or "light smoking".

\section{Smoking terms}

Light smokers: "light smok*" or "non-daily smok*" or "intermittent smok" or "social smok" $^{*}$ or "occasional smok" or "chipper" or "low frequency smok" or "very light smok*" or "non-dependent smok*"

Heavy smokers: "heavy smok*" or "daily smok*" or "dependent smok*" 


\section{Neurocognitive mechanism terms}

General cognitive: "neurocognitive mechanis*" or "neurocognitive measure*" or "neuropsychological" or "neuroimaging" or "cogniti*" or "neural correlate" or "neurocognitive functioning"

Impulsivity: "impulsivity" or "inhibition" or "inhibitory control" or "response inhibition"

Control: "cognitive control" or "executive control" or "executive function*" or "error processing" or "error monitoring" or "conflict resolution" or "performance monitoring" Attention: "Attention" or "attentional bias" or "salienc*"

Reward: "reward" or "reward circuit" or "dopamine"

Motivation: "motivation" or "drive"

Memory: "memory" or "learning" or "learned association"

Craving: "craving" or "cue reactivity" 
Appendix B

IAPS Images 
TABLE B.1: IAPS image number and content label. The mean (SD) valence and arousal ratings presented for each image.

\begin{tabular}{|c|c|c|c|}
\hline Image number & Image content & Valence & Arousal \\
\hline 1410 & Ferret & $7.00(1.45)$ & $4.17(2.02)$ \\
\hline 1440 & Seal & $8.19(1.53)$ & $4.61(2.54)$ \\
\hline 1441 & Polar bears & $7.97(1.28)$ & $3.94(2.38)$ \\
\hline 1450 & Gannet & $6.37(1.62)$ & $2.83(1.87)$ \\
\hline 1505 & Greyhounds & $4.13(1.66)$ & $4.73(1.83)$ \\
\hline 1603 & Butterfly & $6.90(1.48)$ & $3.37(2.20)$ \\
\hline 1610 & Rabbit & $7.69(1.34)$ & $3.08(2.19)$ \\
\hline 1630 & Deer & $7.26(1.48)$ & $4.45(2.45)$ \\
\hline 2039 & Sitting woman & $3.65(1.44)$ & $3.46(1.94)$ \\
\hline 2056 & Diving man & $6.34(1.30)$ & $4.63(1.90)$ \\
\hline 2272 & Child against wall & $4.50(1.78)$ & $3.74(1.94)$ \\
\hline 2273 & Child and bicycle & $5.41(1.55)$ & $3.52(1.81)$ \\
\hline 5030 & Flower & $6.51(1.73)$ & $2.74(2.13)$ \\
\hline 5040 & Venus flytrap & $5.39(1.11)$ & $3.75(1.89)$ \\
\hline 5210 & Sea & $8.03(1.09)$ & $4.60(2.48)$ \\
\hline 5215 & Dockyards & $6.83(1.70)$ & $5.40(2.15)$ \\
\hline 5300 & Space image one & $6.91(1.80)$ & $4.36(2.62)$ \\
\hline 5301 & Space image two & $6.54(1.68)$ & $5.21(2.42)$ \\
\hline 5629 & Mountain & $7.03(1.55)$ & $6.55(2.11)$ \\
\hline 5635 & Snowy street & $6.25(1.56)$ & $3.97(2.03)$ \\
\hline 5725 & Field & $7.09(1.41)$ & $3.55(2.32)$ \\
\hline 5726 & Wheat field & $6.23(1.60)$ & $2.84(2.04)$ \\
\hline 5731 & Blue door & $5.39(1.58)$ & $2.74(1.95)$ \\
\hline 5760 & Flowers & $8.05(1.23)$ & $3.22(2.39)$ \\
\hline 5811 & Pink flowers & $7.23(1.60)$ & $3.30(2.33)$ \\
\hline 5814 & Palm trees & $7.15(1.54)$ & $4.82(2.40)$ \\
\hline 5825 & Sea and rock & $8.03(1.18)$ & $5.46(2.72)$ \\
\hline 5831 & People and beach & $7.63(1.15)$ & $4.43(2.49)$ \\
\hline 5836 & Couple & $7.25(1.39)$ & $4.28(2.41)$ \\
\hline 7012 & Elastic bands & $4.98(1.05)$ & $3.00(1.94)$ \\
\hline 7016 & Razor & $4.76(1.08)$ & $3.40(1.71)$ \\
\hline 7042 & Weights & $5.55(1.23)$ & $4.02(2.26)$ \\
\hline 7045 & Zip & $4.97(0.76)$ & $3.32(1.96)$ \\
\hline 7055 & Bulb & $4.90(0.64)$ & $3.02(1.83)$ \\
\hline 7059 & Hook & $4.93(0.81)$ & $2.73(1.88)$ \\
\hline 7061 & Crossword & $5.40(1.40)$ & $3.66(1.92)$ \\
\hline 7062 & Sowing machine & $5.27(1.06)$ & $3.40(1.94)$ \\
\hline 7081 & Suitcases & $5.36(1.30)$ & $3.96(2.24)$ \\
\hline 7136 & Car clamped & $3.47(1.70)$ & $5.01(2.17)$ \\
\hline 7175 & Lamp & $4.87(1.00)$ & $1.72(1.26)$ \\
\hline
\end{tabular}


Appendix C

\section{Ethics certificates}




\title{
Coventry
University
}

\section{Certificate of Ethical Approval}

Applicant:

\author{
James Bartlett
}

Project Title:

'Investigating Attentional Bias in Nicotine Dependent and Non-dependent Smokers'

This is to certify that the above named applicant has completed the Coventry University Ethical Approval process and their project has been confirmed and approved as Medium Risk

Date of approval:

14 January 2016

Project Reference Number:

P37076 


\title{
Coventry
University
}

\section{Certificate of Ethical Approval}

Applicant:

\author{
James Bartlett
}

Project Title:

Attentional bias towards smoking cues in daily and non-daily smokers

This is to certify that the above named applicant has completed the Coventry University Ethical Approval process and their project has been confirmed and approved as Medium Risk

Date of approval:

01 March 2019

Project Reference Number:

P88261 


\title{
Coventry
University
}

\section{Certificate of Ethical Approval}

Applicant:

\author{
James Bartlett
}

Project Title:

Error Processing and Inhibitory Control in Light and Heavy Smokers

This is to certify that the above named applicant has completed the Coventry University Ethical Approval process and their project has been confirmed and approved as Medium Risk

Date of approval:

30 November 2017

Project Reference Number:

P61175 


\section{Appendix D}

\section{R Packages and References}

afex: Singmann, H., Bolker, B., Westfall, J., Aust, F., and Ben-Shachar, M.S. (2019). afex: Analysis of Factorial Experiments. R package version 0.24-1. https://CRAN . $R$-project.org/package $=$ afex

car: Fox, J. and Weisberg, S. (2019). car: Companion to Applied Regression. R package version 3.0-7. https://cran.r-project.org/web/packages/car/index.html

compute.es: Del Re, A.C. (2013). compute.es: Compute Effect Sizes. R package version 0.2-2. http://cran.r-project.org/web/packages/compute.es

cowplot: Wilke, C.O. (2019). cowplot: Streamlined Plot Theme and Plot Annotations for 'ggplot2'. R package version 1.0.0. https://CRAN. R-project.org/package= cowplot

dplyr: Wickham, H., François, R., Henry, L. and Müller, K. (2019). dplyr: A Grammar of Data Manipulation. R package version 0.8.3. https://CRAN.R-project.org/ package $=$ dplyr

effsize: Torchiano, M .(2019). effsize: Efficient Effect Size Computation. R package version 0.7.6. https://CRAN.R-project.org/package=eff size

emmeans: Lenth, R. (2019). emmeans: Estimated Marginal Means, aka Least-Squares Means. R package version 1.4. https ://CRAN.R-project . org/package=emmeans 
gdata: Warnes, G.R, Bolker, B., Gorjanc, G., Grothendieck, G., Korosec, A., Lumley, T., MacQueen, D., Magnusson, A., and Rogers, J. (2017). gdata: Various R Programming Tools for Data Manipulation. R package version 2.18.0. https://CRAN.R-project. org/package $=$ gdata

gsDesign: Anderson, S. (2016). gsDesign: Group Sequential Design. R package version 3.0-1. https : //CRAN . R-project . org/package=gsDesign

ggplot2: Wickham, H., Chang, W., Henry, L., Pedersen, T.L., Takahashi, K., Wilke, C., Woo, K., Yutani, H. and Dunnington, D. (2020). ggplot2: Create Elegant Data Visualisations Using the Grammar of Graphics. R package version 3.3.0. https: //cran.r-project.org/web/packages/ggplot2/index.html

janitor: Firke, S. (2019). janitor: Simple Tools for Examining and Cleaning Dirty Data. R package version 1.2.0. https://CRAN.R-project.org/package=janitor

kableExtra: Zhu, H. (2019). kableExtra: Construct Complex Table with 'kable' and Pipe Syntax. R package version 1.1.0. https : //CRAN . R-project . org/package=kableExtra

knitr: Xie, Y. (2019). knitr: A General-Purpose Package for Dynamic Report Generation in R. R package version 1.23. https://cran.r-project.org/web/packages/ knitr/index.html

metafor: Viechtbauer, W. (2020). metafor: Meta-Analysis Package for R. R package version 2.4-0. https://cran.r-project.org/web/packages/metafor/index.html

pracma: Borchers, H.W. (2019). pracma: Practical Numerical Math Functions. R package version 2.2.5. https: //CRAN.R-project. org/package=pracma

psych: Revelle, W. (2018) psych: Procedures for Personality and Psychological Research. R package version 1.8.12. https : //CRAN . R-project . org/package=psych 
readbulk: Kieslich, P. J., and Henninger, F. (2016). readbulk: An R package for reading and combining multiple data files. R package version 1.1.2. https : //cran.r-project. org/web/packages/readbulk/index.html

R.matlab: Bengtsson, H. (2018). R.matlab: Read and Write MAT Files and Call MATLAB from Within R. R package version 3.6.2. https ://CRAN . R-project . org/package= R.matlab

splithalf: Parsons, S. (2020). splithalf: Robust estimates of split half reliability. R package version 0.7.1. https://cran.r-project.org/web/packages/splithalf/index. html

tidyr: Wickham, H. and Henry, L. (2019). tidyr: Easily Tidy Data with 'spread()' and 'gather()' Functions. R package version 0.8.3. https://CRAN.R-project.org/ package=tidyr 